\title{
Phenomenological Aspects of Type IIB Flux Compactifications
}

Enrico Pajer

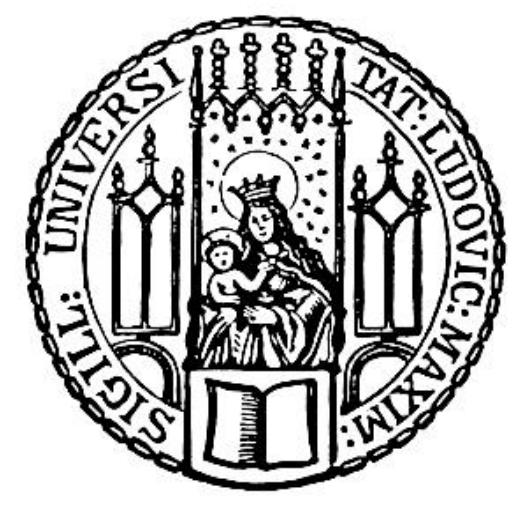

München 2008 



\title{
Phenomenological Aspects of Type IIB Flux Compactifications
}

\author{
Enrico Pajer
}

\author{
Dissertation \\ an der Fakultät für Physik \\ der Ludwig-Maximilians-Universität \\ München \\ vorgelegt von \\ Enrico Pajer \\ aus Venedig, Italien
}

München, den 01. Juni 2008 
Erstgutachter: Dr. Michael Haack

Zweitgutachter: Prof. Dr. Dieter Lüst

Tag der mündlichen Prüfung: 18.07.2008 


\section{Contents}

$\begin{array}{lll}1 & \text { Introduction and conclusions } & 1\end{array}$

2 Type IIB flux compactifications 9

2.1 Superstring theory in a nutshell . . . . . . . . . . . . . . . . . . . . . . . . 10

2.2 How many string theories are there? . . . . . . . . . . . . . . . . . . 13

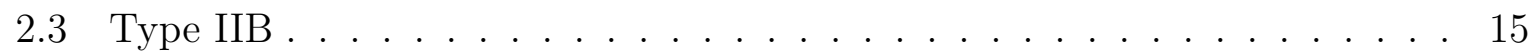

2.4 D-branes . . . . . . . . . . . . . . . . . . . . . . . . . . . . . . . 17

2.5 Flux compactifications $\ldots \ldots \ldots \ldots \ldots$

2.5 .1 The GKP setup . . . . . . . . . . . . . . . . . . . . . . . 19

2.6 Towards de Sitter vacua in string theory . . . . . . . . . . . . . . . . 21

\begin{tabular}{|lll}
\hline & Inflation in string theory & 27
\end{tabular}

3.1 Observations . . . . . . . . . . . . . . . . . . . . . . . . . . . . . . . . . 27

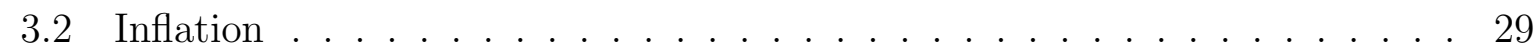

3.2 .1 Slow-roll models . . . . . . . . . . . . . . . . . . . . 32

3.3 String theory models of inflation $\ldots \ldots \ldots \ldots$. . . . . . . . . . . 35

$3.3 .1 \quad$ Open string models . . . . . . . . . . . . . . . . . . . . . 35

3.3 .2 Closed string models . . . . . . . . . . . . . . . . . . . . . . . . . . . . . . . . . . 36

3.4 Discussion $\ldots \ldots \ldots \ldots \ldots \ldots \ldots$

4 Radial brane inflation 39

4.1 Preliminaries . . . . . . . . . . . . . . . . . . . . . . . . . . . . . 40

4.2 The superpotential . . . . . . . . . . . . . . . . . . . . . . . . . . . . . . 42

$4.3 \quad$ Warped Brane inflation. . . . . . . . . . . . . . . . . . . . . . . . . 44

4.3 .1 The $\eta$-problem from volume stabilization . . . . . . . . . . . . . . 44

$4.3 .2 \quad$ F-term potential for the conifold . . . . . . . . . . . . . . . . . . 46

4.4 Critical points of the potential . . . . . . . . . . . . . . . . . . . 47

4.4 .1 Axion stabilization . . . . . . . . . . . . . . . . . . . 48

$4.4 .2 \quad$ Volume stabilization $\ldots \ldots \ldots$. . . . . . . . . . . . . . . . . . 48

$4.4 .3 \quad$ Angular moduli stabilization . . . . . . . . . . . . . . . . . . 50

4.4 .4 Potential with fixed moduli . . . . . . . . . . . . . . . . . . . 51

4.5 Explicit examples: Ouyang vs Kuperstein embedding . . . . . . . . . . . . 52

4.5 .1 Ouyang embedding . . . . . . . . . . . . . . . 52

4.5 .2 Kuperstein embedding . . . . . . . . . . . . . . . . . . . 53

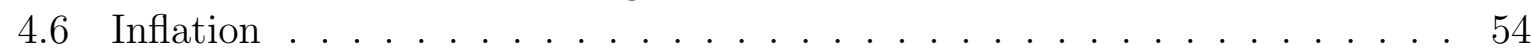


4.6 .1 The effective inflaton potential. . . . . . . . . . . . . . 56

4.6 .2 Inflation through an inflection point . . . . . . . . . . . . . . 58

$4.6 .3 \quad$ Damped oscillatory phase . . . . . . . . . . . . . . . . . . . . . . . . . . . . . . . . . . 59

4.6 .4 Uphill inflation . . . . . . . . . . . . . . . . . . . . . . . . . . . . . . . . . . 61

4.7 Nice upliftings $\ldots \ldots \ldots \ldots$. . . . . . . . . . . . . . . . . . . . . . . . . . . . . . . 63

4.7 .1 Nice downliftings $\ldots \ldots \ldots \ldots$. . . . . . . . . . . . . . . . . . . . . . . . . . . . . . 64

4.8 A summary on radial brane inflation . . . . . . . . . . . . . . 65

$\begin{array}{lll}5 & \text { Angular brane inflation } & 67\end{array}$

5.1 Motivations . . . . . . . . . . . . . . . . . . . 67

5.2 Inflation at the tip $\ldots \ldots \ldots \ldots \ldots \ldots$

5.3 F-term potential for a D3-brane at the tip . . . . . . . . . . . . . 71

5.4 Kuperstein embeddings . . . . . . . . . . . . . . . . . . . . 73

5.4 .1 A simple case . . . . . . . . . . . . . . . . . . . . 74

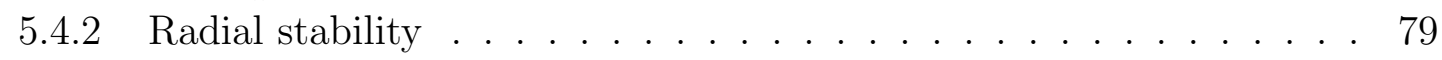

5.5 Inflationary analysis $\ldots \ldots \ldots \ldots \ldots \ldots$

5.5 .1 Slow-roll Natural Inflation . . . . . . . . . . . . . . . . . . . 81

5.5 .2 Slow-roll hilltop inflation . . . . . . . . . . . . . . . . . . 84

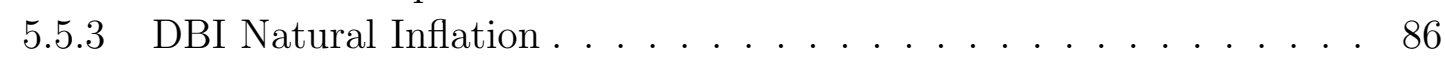

5.6 A no-go result and how to evade it . . . . . . . . . . . . . . . . 92

5.6 .1 Slow-roll-DBI alternation from generic embeddings . . . . . . . . . 93

5.7 A summary on angular brane inflation . . . . . . . . . . . . . 96

6 On soft terms from large volume compactifications 99

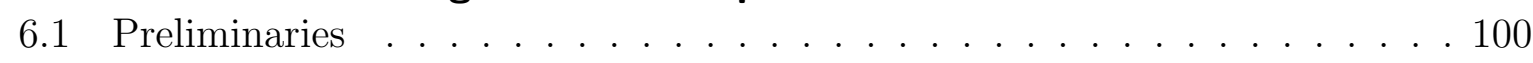

6.2 Review . . . . . . . . . . . . . . . . . . . . . . . . . . . . . 101

6.2 .1 Back to KKLT . . . . . . . . . . . . . . . . . . . . . . 101

6.2 .2 Consistency of KKLT . . . . . . . . . . . . . . . . . . . . . 102

6.2 .3 Large volume scenario (LVS) . . . . . . . . . . . . . . . . . . . . . . . . . . . . . . . . . . . . . . . . . . . . .

6.3 String loop corrections to LVS . . . . . . . . . . . . . . . . 106

6.3.1 From toroidal orientifolds to Calabi-Yau manifolds. . . . . . . . . . 106

6.3 .2 LVS with loop corrections . . . . . . . . . . . . . . . 110

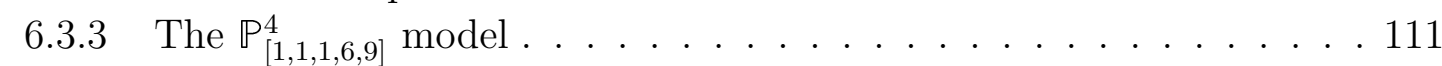

6.4 Gaugino masses . . . . . . . . . . . . . . . . . . . . . . . . . . . 114

$6.4 .1 \quad$ Including loop corrections . . . . . . . . . . . . . . . . 117

6.4 .2 Other soft terms . . . . . . . . . . . . . . . . . 118

6.5 LVS for other classes of Calabi-Yau manifolds? . . . . . . . . . . . . . . . . . 119

6.5 .1 Abundance of "Swiss cheese" Calabi-Yau manifolds . . . . . . . . . 119

6.5 .2 Toroidal orientifolds . . . . . . . . . . . . . . . . 120

6.5 .3 Fibered Calabi-Yau manifolds . . . . . . . . . . . . . . . . . 122

6.6 Further corrections . . . . . . . . . . . . . . . . . . . . . . . . . . . . 122

6.7 A summary on string compactifications . . . . . . . . . . . . . 123 
\begin{tabular}{|l|l}
\hline A The conifold & 125
\end{tabular}

A.1 The singular conifold . . . . . . . . . . . . . . . . . . . . . . . 125

A.2 The deformed conifold . . . . . . . . . . . . . . . . . . . . . 126

B Technical details on radial brane inflation 129

B.1 Dependence of $\tau_{c r}$ on the uplifting and the inflaton . . . . . . . . . . . 129

B.1.1 On $\tau_{c r}$ and the uplifting . . . . . . . . . . . . . . . . . . . . 130

B.1.2 On $\tau_{c r}$ and the inflaton . . . . . . . . . . . . . . . . . . . . . . . . 131

B.2 Sign of $r^{3 / 2}$ term . . . . . . . . . . . . . . . . . . . . 131

B.3 $\quad$ Maximum and minimum of $V(\phi) \quad \ldots \ldots \ldots \ldots$

B.4 Radial brane inflation close to the tip . . . . . . . . . . . . . . . . . . 133

B.5 Forces on D3- and anti D3-branes. . . . . . . . . . . . . . . . . . . . . . . 134

\begin{tabular}{ll}
\hline Technical details on angular brane inflation & 137
\end{tabular}

C.1 Minimization of $\tau$. . . . . . . . . . . . . . . . . . . . . . 137

C.2 Radial stability . . . . . . . . . . . . . . . . . . . . . . 138

C.2.1 Radial stability in the $C \ll B$ regime (DBI inflation) . . . . . . . . 141

C.2.2 Radial stability in the $2 C=B$ regime (slow-roll hilltop inflation) . 141

C.3 From one to many . . . . . . . . . . . . . . . . . . . . . . . 142

C.3.1 Kähler potential. . . . . . . . . . . . . . . . . . . . . . . . . . . 142

C.3.2 Superpotential . . . . . . . . . . . . . . . . . . . . . . . . 143

\begin{tabular}{|ll}
\hline Technical details on soft terms from LVS & 147
\end{tabular}

D.1 Some details on LVS . . . . . . . . . . . . . . . . . . . . . . . . . . . . . 147

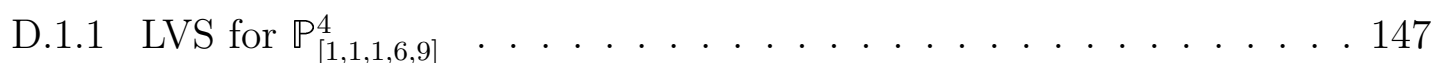

D.1.2 Many Kăhler moduli . . . . . . . . . . . . . . . . . . . . . . . . . . 148

D.2 Loop corrected inverse Kähler metric for $\mathbb{P}_{[1,1,1,6,9]}^{4}$. . . . . . . . . . . . . . 150

D.3 No-scale Kähler potential in type 11 string theory $\ldots$. . . . . . . . . . . . . 151

D.3.1 No-scale structure in type IIA . . . . . . . . . . . . . . . . . . . . . 151

D.3.2 $\quad$ No-scale structure in type IIB . . . . . . . . . . . . . . . . . . . . . 153

D.3.3 $\quad$ Cancellation with just the volume modulus . . . . . . . . . . . . . . . 154

D.3.4 Cancellation with many Kähler moduli . . . . . . . . . . . . . . . . 154

D.3.5 Perturbative corrections to $V_{\mathrm{np} 1}$ and $V_{\mathrm{np} 2}$. . . . . . . . . . . . . . 155

D.4 KK spectrum with fluxes . . . . . . . . . . . . . . . . . . . 156

D.5 The orientifold calculation . . . . . . . . . . . . . . . . . . . . . 159

D.6 Factorized approximation . . . . . . . . . . . . . . . . . . . 162

D.6.1 Factorized approximation of the scalar potential . . . . . . . . . . . 164

$\begin{array}{ll}\text { Bibliography } & 169\end{array}$ 


\section{Summary}

The purpose of this work is to investigate how phenomenologically successful constructions in the field of high energy physics can be embedded into a fundamental theory, and what we can learn from this procedure. In fact, the synergy of an effective and a fundamental approach might be very fruitful. On the one hand, it poses several theoretical challenges to potential candidates for a fundamental theory, which might be ruled out or restricted in their generality. On the other hand, this mutual interplay can inspire new experiments and observations or suggest correlations which are invisible from an effective point of view.

Our starting point is that string theory is entitled to be a valid candidate for a fundamental theory by a series of properties such as the fact that it is a consistent, UV-finite, anomalyfree quantum theory and it describes, in appropriate limits, quantum field theory and general relativity, hence providing a unified description of the four known forces. Within string theory, we choose to work in the framework of type IIB flux compactifications where the issue of moduli stabilization can be successfully addressed. On the phenomenological side, we focus on particle physics and cosmology, and in particular on the MSSM and the mechanism of inflation, respectively.

We construct and study two different models of inflation in string theory. These are models of brane inflation, i.e. models where the primordial exponential expansion of the universe is driven by a scalar field representing the position of a D3-brane (a $3+1$-dimensional dynamical object) in a compact space. We show explicitly that, allowing for fine tuning, a prolonged stage of slow-roll inflation can be achieved during which perturbations are generated in good agreement with the CMB data. In addition, we give an example of the fruitful synergy we mentioned above. One of our two models, namely inflation at the tip, might induce also DBI inflation which produces peculiar features in the spectrum of density perturbations that might be looked for using CMB data.

On the particle physics side, we consider a model, the large volume scenario (LVS), which, among other things, provides a rationale for a low scale susy-breaking. We perform some checks on the consistency of LVS as a string theory model. To this end, we formulate an educated guess for the form of string loop corrections. We then show that physical predictions such as the soft susy-breaking terms are actually surprisingly robust against the inclusion of these corrections. 
Abstract 


\section{Introduction and conclusions}

To motivate string theory phenomenology, which is the subject of the present work, one could start from two different perspectives. The first is incarnated by Plato, the person on the left part of figure 1.1. From this perspective, one should start introducing and discussing the "theory of Forms", which in the present context is to be identified with string theory! In a second step, one should descend to our "world of phenomena", which is populated not by the Forms themselves, but by their shadows, which in the present discussion should be identified with the physical phenomena. The second perspective is incarnated by Aristotle ${ }^{2}$, depicted on the right part of figure 1.1. Now, the starting point are experiments and observations, from which one should collect a series of empirical facts. Then, one should try to construct a simple and elegant explanation to account for these facts. The explanation might have some implications which can in principle be tested. A successful and logically consistent explanation might eventually be promoted to the status of predictive theory.

We are going to embrace both perspectives. First, we are going to discover the remarkable properties of string theory, which promoted it to a very active research field. Second we are going to take a bird's-eye view on some outstanding open phenomenological issues in high energy physics.

Let us start with Plato's perspective. Since the early seventies, the intense efforts dedicated to the formulation and study of string theory have been crowned by several major developments. The fact that string theory is a quantum theory which provides a consistent UV completion of general relativity has played a key role in qualifying it as a candidate for a fundamental theory. All four known fundamental forces can be described within a unique theory, which, in a certain limit, reduces to quantum field theory and general relativity. In string theory, situations outside the regime of validity of these theories can in principle be investigated. Before we come to those ideas and constructions that will play a major role in this work, in passing we would like to mention, without any pretense of completeness, some significant theoretical breakthroughs.

The string theory description of systems dominated by gravity, notably black holes, encodes information about the microstates of the system. Remarkably, the resulting micro-

\footnotetext{
${ }^{1}$ As the reader will notice, in this introduction we do not give references to the literature, mainly because this is meant to be an invitation to the present work accompanied by a general discussion of some important ideas in the context of high energy physics.

${ }^{2}$ The cultivated reader might remember that Aristotle was Plato's student. By no means this should be interpreted as a hint to prefer one approach to the other.
} 


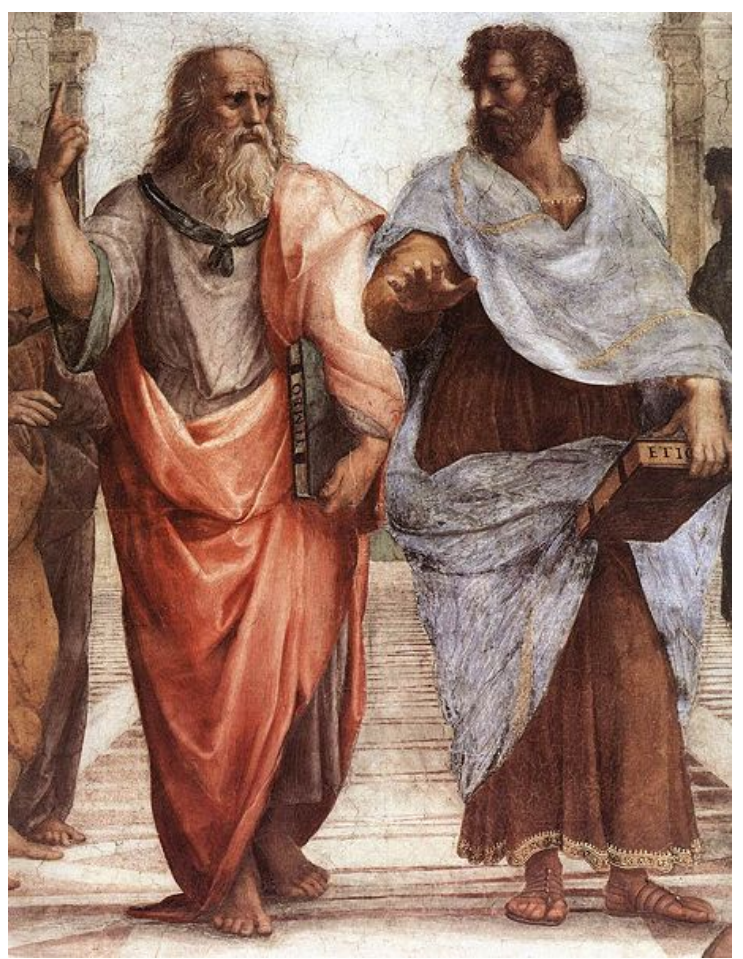

Figure 1.1: The figure [1] depicts Plato (left) and Aristotle (right). The former gestures to the heavens, representing his belief in the (theory of) Forms, whilst the latter gestures to the earth, representing his belief in empirical knowledge, obtained through experiments and observations.

scopic entropy correctly matches the expected macroscopic entropy obtained from quantum field theory and general relativity considerations. This extraordinary feature has strengthened the belief that we can learn a big deal about quantum properties of gravity from the study of black holes in string theory. This has in fact become an active research field and a promising direction to understand the structure of and the basic principle behind quantum gravity. On the other hand, the descent is still very long, from the "world of Forms" where string theory black holes live to the "world of phenomena "where their shadows, i.e. the astrophysical black holes can be observed.

Another celebrated breakthrough is the AdS/CFT correspondence, i.e. the idea that a theory of gravity is dual to a gauge theory (in lower dimensions). Just listing the many contexts in which AdS/CFT has been employed would take us already too far from our main focus. Hence we restrict ourselves to comment on the application of AdS/CFT to the description of strongly coupled gauge theories, which does make its way through to phenomenology. The quark-gluon plasma is a state of matter where, except for the dual gravitational description provided by the correspondence, no other analytic tool is available. Notice that this connection from string theory to phenomenology is deeply different from the one we will consider in the rest of the present work. AdS/CFT is used as a technical tool to perform calculations in the quark-gluon plasma, for which a consistent UV finite theory, namely QCD, is already available, but it is not manageable 
to get analytical control.

We now come to the main focus of this work, string cosmology and string particle phenomenology. Let us begin discussing the former. String theory is in principle suitable to describe the physics of the very early universe, even when the spacetime curvature was so large as to exceed the regime of validity of general relativity. Models have been proposed, which try to address the problem of the initial singularity of the big bang, such as pre-big bang theories, bouncing cosmologies, and so on. Unfortunately, a large part of our knowledge of string theory is based on the perturbative approach and it is therefore hard to handle high curvature and high energy regimes where the perturbative expansion breaks down. This is why the straightest path to phenomenology passes through phenomena which can be described by perturbative string theory and at the same time are relevant to understand cosmological observations. A prominent example, which will in fact occupy us for a large part of this work, is cosmological inflation (see chapter 3 ).

In string theory, gauge theories are automatically built-in. Unfortunately, the low energy effective action is not characterized by one particular gauge theory, instead a host of different gauge theories arise by expanding around the many existing vacua. This situation has justified the idea of a landscape of vacua, whose consequence is that string theory may lead to many different effective physical laws. The presence of a landscape obliges us to face the following alternative: either we are contented by finding at least one vacuum that describes our universe and we renounce to explain why this has been chosen among others or we resort to statistical methods (and possibly invoke anthropic principles) and try to estimate the probability with which the features we observe occur in the landscape. In this work we follow the first approach, i.e. we search the landscape for a model that describes our world even at the cost of making some non-generic assumptions and/or restricting ourselves to particular regions of it. The suspicious reader might object that this pretty much resembles the effective field theory approach, in the sense that some (maybe many) quantities have to be determined by experiment and can not be predicted a priori. Nevertheless, a considerable potential advantage with respect to effective theories is that certain low energy features might be correlated by the higher energy physics, i.e. due to consistency requirements of string theory.

Let us sidesteps from the left to the right part of figure 1.1 and switch to Aristotle's perspective. We should start by considering remarkable empirical facts. We restrict ourselves to the domain of high energy physics, and to those facts which are still elusive to an explanation in terms of well established theories such as the Standard Model and general relativity. The last century has witnessed the birth of quantum field theory and its extraordinary success, incarnated by the Standard Model, in describing particle physics experiments. Two parts of the Standard Model where most questions are still open are the neutrino and the Higgs sectors. The former is a young sector in the sense that it is only in the last decade that we have collected convincing evidence that neutrinos possess masses which are not accounted for in the Standard Model. The latter is as old as the Standard Model itself, and it is expected it will be the arena of important improvements, 
driven by the incoming LHC experiments. In addition, several features of the Standard Model are particularly ad hoc, which is considered a hint that a new paradigm in particle physics might become necessary, even very soon, at energies comparable to those of LHC experiments.

Among the many extensions of the Standard Model, a popular one is the minimal supersymmetric Standard Model (MSSM) which introduces a new spacetime symmetry relating bosons with fermions. Together with nice features such as gauge coupling unification (suggesting the idea of a great unified theory), stabilization of the weak scale (hierarchy problem) and possible dark matter candidates, the MSSM introduces a host of new parameters which are a priori undetermined. It would be desirable to find a fundamental theory where the origin of these parameters is explained and possibly some correlations are imposed by consistency. This is possible in string theory and we will see an explicit example in chapter 6, where we calculate, in a particular model know as the large volume scenario (LVS), some of the soft supersymmetry breaking terms (constituting most of the MSSM parameters).

In addition to particle physics experiments, also cosmological and astrophysical observations provide data relevant for high energy physics. The last decade has been as quiet and quiescent for experimental particle physics as it has been fruitful and exciting for observational cosmology (see chapter 3). Several evidences have been accumulated in favor of the so called $\Lambda$ cold dark matter model $(\Lambda \mathrm{CDM})$, consisting of a flat universe with critical total energy density, of which $72 \%$ behaves as dark energy, $23 \%$ as dark matter and the remaining $4 \%$ as baryonic matter. This picture implies that we lack an understanding for most of the energy of the universe. Even more interestingly from the high energy point of view, in the last decade the WMAP data about the cosmic microwave background radiation (CMB for short) have become available, which enormously improve the date of the COBE experiment.

The existence of the CMB supports the big bang theory but also makes more acute the initial condition problems that afflict it. A very popular possibility to improve on these issues is to assume a period of exponential inflation in the early universe (see chapter 3). Unexpectedly and remarkably, inflation provides a natural and elegant mechanism to seed the inhomogeneities which are eventually responsible for the formation of the large scale structures we observe in the universe. The success of inflation raises some fundamental questions such as for example what is responsible for the exponential expansion and how does the inflationary phase connect with the hot big bang. It would be desirable to address these and other questions concerning inflation in the framework of a fundamental theory. This constitutes one of the main motivations of the present paper; in particular in chapters 4 and 5 we will construct and study models of inflation in string theory.

We conclude here our discussion of the motivations for and the perspectives on string theory phenomenology. We continue with an overview of the state of the art of constructing cosmological and particle physics models in string theory. This is obviously a vast 
subject, and in fact we will make a selection of models and ideas relevant for the rest of the paper. The following overview should serve as a map to help the reader's orientation when we will present and discuss the various parts and results of this work.

For many years, most efforts devoted to embed the Standard Model in string theory concentrated on the heterotic models. More recently, considerable progress has been made in type II theories. On the type IIA side, intersecting D6-branes are the most popular setup, where each stack hosts a factor of the Standard Model gauge group. In type IIB, related to type IIA by T-duality, the Standard Model could be constructed on magnetized D7 branes. Given that string theory possesses a target-space supersymmetric description, the supersymmetric extension of the standard model, i.e. the MSSM, can naturally be allocated. One of the key questions in this type of models is which mechanism is responsible for the susy-breaking. In particular, the relevant quantities which are directly connected to experiments are the soft susy-breaking terms. Later in this work we will encounter several mechanisms to break supersymmetry in type IIB constructions. One is an anti D3-branes which, in a background with imaginary self-dual fluxes, breaks the residual $\mathrm{N}=1$ supersymmetry. The susy-breaking is somehow explicit, in the sense that, in addition to F- and D-terms, another non-supersymmetric contribution to the potential is present. We will consider this possibility in the cosmological context, where our focus will be more on the vacuum energy than on low energy particle spectra. Another possibility is spontaneous susy-breaking, i.e. when F- and D-terms do not satisfy the susyconditions in a particular vacuum. A model, known as large volume scenario (LVS), where this mechanism is at work will be presented in chapter 6, where we will calculate some of the soft susy-breaking terms. An interesting feature of LVS is that it can provide a rationale for a low scale susy-breaking.

On the string cosmology side, we restrict our overview to inflationary models, which will be the focus of chapters 4 and 5. A simple, popular and phenomenologically successful effective model to induce a primordial exponential expansion consists of a single scalar field with a relatively flat potential. Generically, the procedure of compactifying to lower dimensions produces massless scalar fields, which correspond to the deformations of the compact manifold. These massless fields, usually called moduli, are clearly troublesome for particle phenomenology because no massless scalar has ever been observed, and actually quite strong bounds are available on the long-range force that moduli would induce (sometimes called fifth force). On the other hand moduli are a blessing for cosmology because they might be responsible for a stage of inflation. A strategy to reconcile string theory with low energy physics is flux compactification. In this approach, the dimensional reduction is performed in the presence of fluxes threading various cycles of the compact space. In the resulting lower-dimensional effective action, the moduli $3^{3}$ acquire a mass because the deformations which they correspond to are constrained by the fluxes. Given

\footnotetext{
${ }^{3}$ Strictly speaking, once they become massive, they should not be called moduli anymore. We will nevertheless continue using this terminology, to avoid cumbersome expressions like "the would be moduli in the fluxless case".
} 
this picture, the inflationary phase might result from the evolution of the moduli towards their minima.

We will restrict our considerations to type IIB, because flux compactifications and the stabilization of all the moduli $\mathrm{l}^{4}$ are best understood there. For a typical compactification we expect $\mathcal{O}(100)$ moduli corresponding to volumes and shapes of the Calabi-Yau cycles. In addition to these closed string moduli, there might also be open string moduli corresponding to the positions of D-branes in the compact space. The generic expectation is therefore a complicated multi-field dynamics which presents obvious obstacles to both an analytical and numerical approach. A way to sidestep this technical difficulty, is to make some non-generic assumptions, e.g. that one of the moduli is much lighter than all the others and therefore, after the latter have reached their minima, the former is the only dynamical field. The inflationary analysis reduces to the study of the effective single-field potential for the candidate inflaton. Both closed and open string moduli might play the role of the inflaton, but in this work we consider only the second possibility. Open string inflation, commonly known as brane inflation, in type IIB is typically (but not necessarily) driven by the position modulus in the compact space of a spacetime-filling D3-brane. In this setup, several phenomenological and theoretical questions related to inflation can be addressed, such as which perturbations are produced, how the inflaton couples to the Standard Model sector, how reheating takes place, whether there are other observable consequences like cosmic strings, and so on.

Now that we have briefly reviewed some approaches to embed inflation and the (MS)SM into string theory, we are ready to discuss the achievements of the present work and to put them into the context. After introducing string theory in chapter 2, with particular focus on type IIB phenomenological constructions, and after describing inflation in chapter 3, we dedicate chapters 4 and 5 to a thorough study of two models of brane inflation. The goal is to construct, in a controlled flux compactification, a model where the potential felt by the D3-brane is suitable for inflation. In chapter 4 we consider a warped conifold as local approximation to the Calabi-Yau manifold; at the tip of the conifold an anti D3-brane is located, which attracts the D3-brane, uplifts the vacuum energy to positive values, and breaks supersymmetry. The attractive brane-anti brane force, thank to the warping, would be of the slow-roll type. Unfortunately, the D3-brane also feels other forces which originates from the gravitational backreaction. The stabilization of the closed string moduli induces a large mass for the inflaton and prevents slow-roll inflation. We investigate the possibility of cancelling this effect using threshold corrections to the non-perturbative superpotential. We show that, with an appropriate fine tuning of the parameters, a potential with a flat inflection point (see figure 4.1) arises, which induces a prolonged stage of inflation.

In chapter 5, we construct a different model, that we call inflation at the tip, where no anti D3-brane is present and the motion of the D3-brane takes place along the tip of a warped deformed conifold. This is the first model of brane inflation where the inflaton potential

\footnotetext{
${ }^{4}$ Possibly also recurring to non-perturbative effects, as we will see in section 2.6
} 
is generated exclusively by moduli stabilization effects (once threshold corrections are taken into account). We argue that this, as opposed to brane-anti brane inflation, is in fact the most natural case. We show that inflation at the tip can drive slow-roll inflation, provided that some fine tuning is performed, and it can produce perturbations in excellent agreement with CMB data. Another interesting feature of this model is that the DBI regime (see section 5.5.3) can easily be achieved. We argue that in certain cases, a prolonged stage of inflation can be achieved together with perturbations of the DBI type, i.e. which possess features experimentally distinguishable from the slow-roll case. In addition, inflation at the tip poses a theoretical challenge regarding the mechanism of reheating. Summarizing, we have found two phenomenologically successful models of brane inflation and we have described their respective features. On the other hand, in the analysis producing the above results, we have neglected several corrections, of stringy and/or quantum field theory origin, the reason being that they are mostly unknown. To improve on this issue and increase the control we have on phenomenological models, we dedicate chapter 6 to the study of the role of quantum corrections.

For this study, we decided to leave the realm of string cosmology and enter the area of string particle phenomenology. In fact, we consider one of the aforementioned approaches to embed the MSSM into string theory. To be more precise, we investigate a particular type IIB setup, known as the large volume scenario (LVS), where all moduli can be fixed and some of them to hierarchically large values. The vacuum which is obtained breaks supersymmetry and has several interesting phenomenological properties. One example is a low susy-breaking scale, whose low value has a geometrical origin. We perform a series of checks on the self-consistency of this model as a string theory model as opposed to a supergravity model. We discover that, although some corrections, neglected in LVS, a priori seemed dangerous, a detailed investigation reveals that they only induce small quantitative changes. In particular, we calculate the effects of string loop corrections to gaugino masses (and comment on other soft terms); we verify that the tree level result is actually very robust and gets only slightly modified by the corrections. Our is just a modest step, because a part from string loop corrections, several other effects are also potentially dangerous (see later). Nevertheless, our results are encouraging and should stimulate further efforts to try to assess the consistency of phenomenological scenarios as bone fide string constructions. 


\section{Type IIB flux compactifications}

The purpose of this chapter is to review the main steps that lead from the NambuGoto action to phenomenologically interesting string theory models. The review will favor deepness to wideness in the sense that we focus our attention on the ideas and constructions that play a crucial role in type IIB flux compactifications and mention only shortly alternative setups.

We start with the world-sheet formulation of string theory and describe its dual targetspace interpretation. We then consider the massless spectrum and stress that it contains graviton-like excitations. After briefly mentioning the various types of string theories and how they could be unified by M-theory, we concentrate on type IIB. We describe the massless spectrum and the 10-dimensional low energy effective action both for closed strings and, after introducing D-branes, for open strings. We explain the idea of flux compactification and review which 4-dimensional effective action follows. Finally we show how all moduli can be fixed in a vacuum that breaks supersymmetry and have a cosmological constant which is tunable, at least in principle.

We do not review how one can try to embed the Standard Model in this construction. Useless to say that this is a formidable task; fortunately for most of the issues we will be interested in, we will not need to be very detailed about it. This is because both inflation (chapters 4 and 5) and supersymmetry breaking (chapter 6) are supposed to take place in a different sector from that of the SM. The assumption, that has to be carefully verified, is that the SM sector provides quantitative but not qualitative corrections to dynamics of these sectors. Once this dynamics is established for the isolated sector, the next step is to investigate how it manifests itself in the visible SM sector.

A last preliminary comment about the goal of the present work is in order. As we discussed in the introduction, several hints seem to indicate that string theory has a very large number of vacua, each one describing very different low energy physics. Our goal is to look for those vacua whose phenomenology can describe our world. This justifies several assumptions we will make, which are non-generic but valid in some regions of the landscape. The issue of how one particular vacuum is selected is different in nature and will not be addressed here. 


\subsection{Superstring theory in a nutshell}

In this section we review the basic steps to obtain the spectum of superstring theories; we stress that string theory provides an ultraviolet-finite completion of general relativity (GR) and that a graviton-like excitation appears in the closed string spectrum.

Our starting point is a two-dimensional supersymmetric field theory determined by the action

$$
S=\frac{1}{4 \pi} \int d \sigma d \tau\left(\frac{1}{\alpha^{\prime}} \partial_{\alpha} X^{\mu} \partial^{\alpha} X_{\mu}-i \bar{\psi}^{\mu} \rho^{\alpha} \partial_{\alpha} \psi_{\mu}\right)
$$

where $\sigma$ and $\tau$ are real coordinates that parameterize a two dimensional Riemann surface, possibly with boundary, called the string world-sheet. The $2 \times 2$ matrices $\rho^{\alpha}$ with $\alpha=0,1$ are the two-dimensional gamma matrices. $X^{\mu}$ with $\mu=0, \ldots, D-1$ are $D$ real scalar fields on the two-dimensional world-sheet, $\psi^{\mu}$ are the supersymmetric partners of $X^{\mu}$, i.e. they are world-sheet fermions obtained by acting with a supersymmetric generator on the $X^{\mu}$ bosonic operators 1 . Finally, $\alpha^{\prime}$ is a parameter of the theory, sometimes referred to as Regge slope, with the dimension of a length squared.

It turns out that the quantum theory obtained quantizing this action has an anomaly (of the Weyl invariance) that makes it inconsistent in all but $D=10$ dimensions ${ }^{2}$. From now on we focus on this number of target-space dimensions, usually called the critical dimension.

The action (2.1) has an $S O(1,9)$ global (internal from the world-sheet point of view) symmetry. This observation leads to an interesting interpretation of the action (2.1): the fields $X^{\mu}$ give the embedding of the two-dimensional world-sheet in a 10-dimensional target space as depicted in figure 2.1. In other words, the action (2.1) describes the propagation of a 1-dimensional extended object (the string) in the 10-dimensional spacetime. The fields $\psi^{\mu}$ describe additional degrees of freedom of the string beyond the geometrical oscillations described by $X^{\mu}$. As it is common for non-linear sigma models, of which string theory is an example, we can think of the system described by (2.1) from two equivalent points of view: as a two-dimensional field theory on the world-sheet or as a theory of strings propagating in a 10-dimensional spacetime.

The first striking feature on which we want to comment and which gives one of the strongest motivations to study string theory is that the action (2.1) describes, among

\footnotetext{
${ }^{1}$ In two dimensions we can perform both Majorana and Weyl projections at the same time; a Majorana spinor has two real components, that in an appropriate basis are right and left handed spinors, respectively.

${ }^{2}$ This comment refers to quantization around a constant dilaton background. Another background can lead to different values of $D$ for which the Weyl anomaly is absent. In fact exact solutions exist, but they describe a spacetime physics where the laws of nature vary strongly in space and time in contradiction with observations (e.g. the equivalence principle).
} 


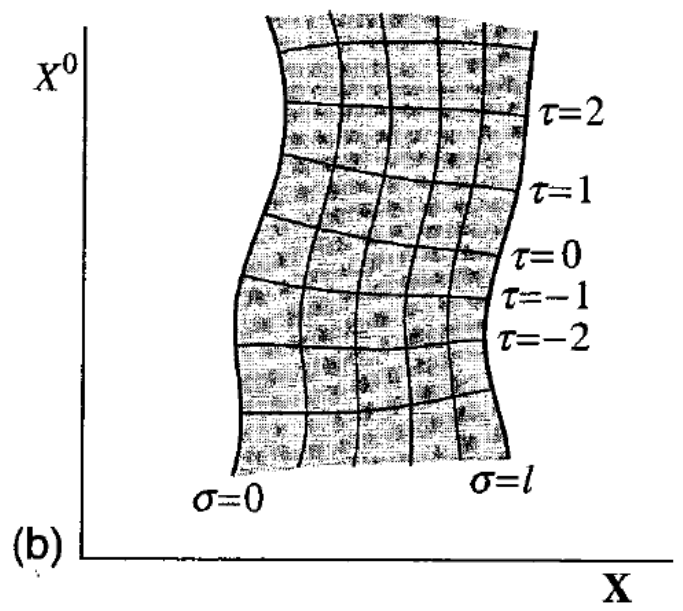

Figure 2.1: The bosonic coordinates $X^{\mu}$ describe the embedding of the world-sheet in the 10dimensional target-space. The world-sheet coordinate $\tau$ describes the evolution of the shape of the string as it moves in the target-space. It can be thought of as a time-like coordinate. This figure is taken from [2].

other things, the dynamics of gravitons in the target space. Let us briefly see how this comes about. As we said we look at the fields $X^{\mu}(\tau, \sigma)$ as the embedding of a string in the 10-dimensional target-space. Following our intuition, we expect that the degrees of freedom of such an object are oscillations plus the motion of the center of mass of the string. Which oscillations are allowed depends crucially on the boundary conditions that we impose, i.e. if the string is open or closed. From the world-sheet point of view this means that we have to decide if the world-sheet has a boundary, corresponding to the end points of an open string, or periodic boundary corresponding to the case of a closed string.

In analogy with the string of a violin, complicated oscillation of a string can be decomposed in Fourier modes. The more irregular the shape of the string during its oscillations is, the higher Fourier modes are excited. Each mode has an associated energy, that could be e.g. obtained by looking at the Hamiltonian of the system.

The periodic boundary conditions that describe a closed string allow for the existence of right- and left-handed modes moving independently along the string. The independence of these two sectors plays a crucial role in the construction of different string theories. On the other hand the open string boundary condition relates left- and right-moving oscillations so that effectively an open string has only half of the oscillation modes of a closed string. The Majorana spinor $\psi^{\mu}$ has two independent components in the closed string case. In an appropriate basis they describe right- and left-moving degrees of freedom and we will refer to them as $\psi_{+}^{\mu}$ and $\psi_{-}^{\mu}$, respectively.

It is very useful to think of the effective action, i.e. the action describing the degrees of freedom which are relevant for the system under consideration. Relevant in our case 
means light because in reasonable physical situations the degrees of freedom that can be excited are those whose energy is not much larger than the typical energy available to the system. This motivates us to look at the lowest energy oscillations.

The process of quantization that leads to the spectrum of string theory can be found in standard textbooks [2, 3, 4, [5, 6]. We will not review it here but just mention those steps which are relevant for the present work. We concentrate on closed strings because there we will find a graviton-like excitation. Before we Fourier expand and subsequently quantize the fields $\left\{X^{\mu}, \psi^{\mu}\right\}$ we need to explicitly specify the boundary conditions. For $X^{\mu}$ this is easily done imposing periodic boundary conditions $X^{\mu}(\tau, 0)=X^{\mu}(\tau, l)$ where $l$ is the length of the closed string. On the other hand, for the right-moving world-sheet fermionic field $\psi_{+}^{\mu}$, two choices are allowed:

$$
\begin{array}{rr}
\text { Ramond (R) : } & \psi_{+}^{\mu}(\tau, 0)=+\psi_{+}^{\mu}(\tau, l) \\
\text { Neveu Schwarz (NS) : } & \psi_{+}^{\mu}(\tau, 0)=-\psi_{+}^{\mu}(\tau, l)
\end{array}
$$

and the same for $\psi_{-}^{\mu}$. The quantization process in the NS sector leads to a ground state that is a singlet under the target space Lorenz symmetry, i.e. a scalar. On the other hand, the $\mathrm{R}$ choice leads to a degenerate vacuum state. It can be seen that the set of vacua in the $\mathrm{R}$ sector forms a representation of the target-space gamma matrices, leading to the interpretation of it as a target-space fermionic state.

The independence of right and left moving sector is valid also for the choice of boundary conditions. Therefore, we have four possibilities for $\left\{\psi_{+}^{\mu}, \psi_{-}^{\mu}\right\}$ that we will denote with NS-NS, R-R, NS-R and R-NS sector $3^{3}$. Remember that in the R sector there are fermionic states while in the NS sector there are bosonic states. We deduce from this that a closed string has two bosonic sectors, R-R and NS-NS, and two fermionic sectors, R-NS and NS-R.

It will be useful to specify another property of the string states, i.e. their world-sheet fermionic number. This is defined mod 2 and it is equal to +1 if the state is obtained acting with an odd number of world-sheet fermionic operators on the vacuum, while it is equal to -1 otherwise. The states in each sector ${ }^{4}$ together with their world-sheet fermionic number are resumed in figure 2.2 .

The spin 2 tensor state, i.e. the $(2)=\mathbf{3 5}$ in figure 2.2, in the NS-NS sector is the soughtfor graviton-like excitation. It can be seen, (e.g. calculating its vertex operator) that this oscillation couples to the spacetime energy momentum tensor of the $\left\{X^{\mu}, \psi^{\mu}\right\}$ system exactly as required by general relativity. This is a key feature of string theory because gravity is automatically included in the quantized theory. Another way to see this is to calculate the $\beta$-functions for the couplings on the world-sheet. The requirement of

\footnotetext{
${ }^{3}$ This is not the case for an open string because as we said before, the right and left moving excitations are not independent. Hence an open string has just two sectors: R and NS.

${ }^{4}$ We neglect the NS sector with a negative world-sheet fermionic number, which contains a tachyon, because it is projected out by the GSO projection, as we will see later.
} 


\begin{tabular}{|c|c|c|c|c|c|}
\hline sector & $S O(8)$ spin & & tensors & & dimensions \\
\hline$(\mathrm{NS}+, \mathrm{NS}+)$ & $\mathbf{8}_{v} \times \mathbf{8}_{v}$ & $=$ & {$[0]+[2]+(2)$} & $=$ & $1+28+35$ \\
\hline$(\mathrm{R}+, \mathrm{R}+)$ & $8 \times 8$ & $=$ & {$[0]+[2]+[4]_{+}$} & $=$ & $1+28+35_{+}$ \\
\hline$(\mathrm{R}+, \mathrm{R}-)$ & $\mathbf{8} \times \mathbf{8}^{\prime}$ & $=$ & {$[1]+[3]$} & $=$ & $\mathbf{8}_{v}+56_{t}$ \\
\hline$(\mathrm{R}-, \mathrm{R}-)$ & $\mathbf{8}^{\prime} \times \mathbf{8}^{\prime}$ & $=$ & {$[0]+[2]+[4]_{-}$} & $=$ & $1+28+35$ \\
\hline$(\mathrm{NS}+, \mathrm{R}+)$ & $\mathbf{8}_{v} \times \mathbf{8}$ & & & $=$ & $8^{\prime}+56$ \\
\hline$(\mathrm{NS}+, \mathrm{R}-)$ & $\mathbf{8}_{v} \times \mathbf{8}^{\prime}$ & & & $=$ & $8+56^{\prime}$ \\
\hline
\end{tabular}

Figure 2.2: The massless spectrum of the various closed string sectors. Table taken from [4].

conformality, i.e. $\beta=0$, is equivalent to the Einstein's Equations (EE's) in the targetspace. Even more strikingly the theory has no ultraviolet divergences that typically plague many attempts to directly quantize general relativity.

The fact that string theory provides an ultraviolet completion of general relativity will be very important for the present work when we will study inflation. In section 3, after reviewing the idea of inflation, we will argue that the successes of this mechanism raise to several fundamental questions that due to their nature can be addressed only in the context of a fundamental theory. As inflation is a gravitational effect, it is essential that the candidate fundamental theory includes gravity. String theory is up to now the most developed quantum theory that contains gravity; it is therefore natural to see what can we learn from trying to embed inflation in string theory.

\subsection{How many string theories are there?}

In this section, we review the possible consistent string theories and comment on the conjecture that they are different limits of a unique theory called M-theory. The focus of the present work is on a particular "corner" of M-theory: type IIB.

Even restricting ourselves to just closed strings, not all the sectors that we found in the last chapter can be put together to form a consistent string theory. In fact it turns out that fundamental features like modular invariance, closure of the operator product expansions, locality, the absence of tachyons and target space supersymmetry require to truncate the spectrum of table 2.2. In the Ramond-Neveu Schwarz approach that we sketched in the last section, this is achieved performing the Gliozzi-Scherk-Olive projection (GSO) $)^{5}$. This consists in keeping only those states with a definite world-sheet fermion number. It comes

\footnotetext{
${ }^{5}$ Another popular approach where target-space supersymmetry is explicit from the beginning was developed by Green and Schwarz [5]
} 
out that there are two possible choices defining two different (but as we will see related) string theories

$$
\begin{array}{ll}
\text { type IIB } & (-1)^{F_{L}}=(-1)^{F_{R}}=+1 \\
\text { type IIA } & (-1)^{F_{L}}=+1, \quad(-1)^{F_{R}}=\left\{\begin{array}{ll}
-1 & R \\
+1 & N S
\end{array},\right.
\end{array}
$$

where $F_{L}$ and $F_{R}$ are the world-sheet fermion number operators in the left- and rightmoving sector, respectively. The "II" refers to the fact that these closed string theories have two gravitinos in the massless spectrum, i.e. they have $N=2$ supersymmetries in 10-dimensions. In the present work we concentrate on type IIB string theory; a detailed description of this theory is postponed to the next section.

For completeness, we mention that, a part from type IIA and IIB, there are other three consistent string theories. One of them is obtained by adding to the above closed string spectrum also open strings and performing the truncation. This theory is called type I, where "I" refers to the fact that it has $\mathrm{N}=1$ supersymmetry in 10-dimensions. For open strings there are two choices of boundary conditions for the world-sheet bosonic coordinates as opposed to the unique choice of periodic boundary conditions in the closed string case. One can impose Dirichlet or Neumann boundary conditions corresponding to the case when an end of the string is free to move or is kept fixed at a certain point 6 .

Another string theory is the bosonic string theory where fermions are absent; this theory has a tachyon (a particle with negative mass) which makes it unstable. One can interpret this instability as the fact that we have quantized the theory around a background which was a maximum instead of a minimum. This idea led to the conjecture that, following the condensation of the tachyon, eventually a stable minimum will be reached. Although this can hardly be checked using the first quantized approach to string theory that we describe in this chapter, a certain amount of evidence that supports this conjecture has been obtained by a second quantized approach to string theory called string field theory.

Finally, two further consistent string theories called $S O(32)$ and $E_{8} \times E_{8}$ heterotic string theory, respectively, are obtained treating differently the right- and the left-moving sector of the closed string. One sector is analogous to the superstring while the other is analogous to the bosonic string.

Interestingly, it has been realized that the five superstring theories (type IIA, IIB, I and heterotic $S O(32)$ and $\left.E_{8} \times E_{8}\right)$ are related by a series of dualities. This has led to the conjecture that they are only different limits of the same theory which has been called M-theory. Although considerable efforts have been devoted to construct such a unique theory we are still missing an explicit formulation. What we can do is to start studying the various corners of M-theory, as depicted in figure 2.3. In the present work we will focus on the type IIB corner because, as we will see, issues like moduli stabilization are much more developed in this framework than in others.

\footnotetext{
${ }^{6}$ In section 2.4 we will see that open string are attached, in the case of Dirichlet conditions, to dynamical,
} 


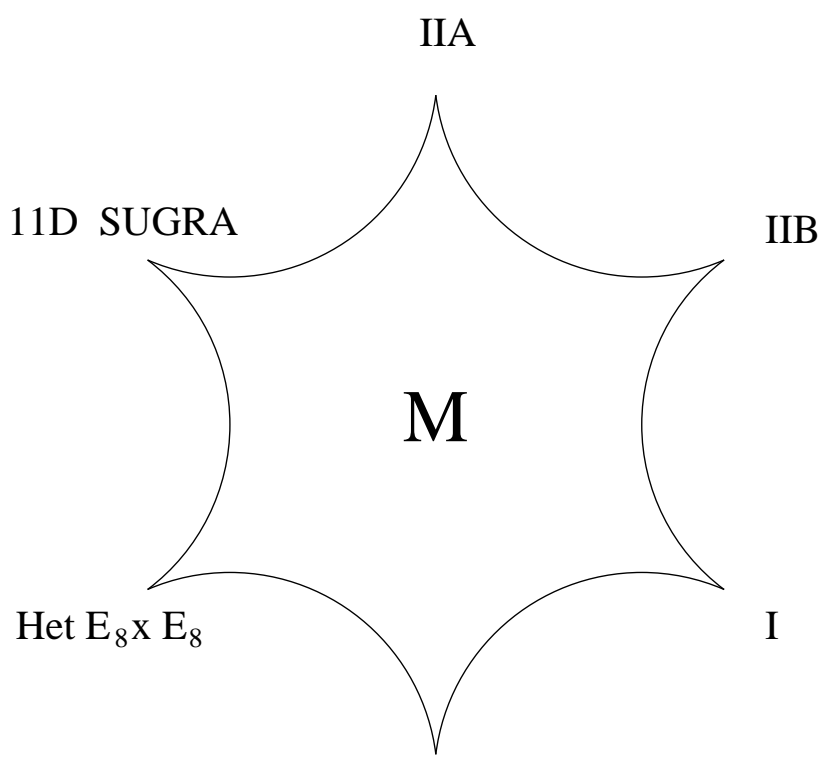

Het $\mathrm{SO}(32)$

Figure 2.3: The M-theory cartoon: the 5 known superstring theories and $d=11, N=1$ sugra are conjectured to be different limits of the same unique theory. Figure taken from [7].

\subsection{Type IIB}

In this section we write down and discuss the 10-dimensional low energy effective action for the massless spectrum of type IIB superstring theory.

As we said, one of the five consistent string theories is type IIB, which is obtained by quantizing the action (2.1) keeping only closed strings and performing the GSO projection described in (2.4). Looking at the single closed string spectrum, we discover that the first excited state beyond the massless ones has a mass of order $m_{s}=\left(\alpha^{\prime}\right)^{-1 / 2}$. We will refere to $m_{s}$ as the string scale.

If the typical energy available to the system under investigation is much smaller than $m_{s}$, then we are allowed to integrate out all heavier modes and concentrate on the massless spectrum. This will be our working assumption both in the context of particle physics and of cosmological inflation. We will see in the next chapter that if the scalar perturbations observed in the cosmic microwave background radiation (CMB) are originated by oscillations of the inflaton, then observations bound the scale of inflation to be lower than about $10^{16} \mathrm{GeV}$. Of course the phenomenologically interesting energy scale for particle physics is much lower. It will therefore suffice for us to assume that $m_{s}$ is much higher than $10^{16}$ $\mathrm{GeV}$ to legitimate the use of just the massless spectrum of string theory throughout the present work.

non-perturbative objects called D-branes. 
The massless spectum of type IIB is obtained adding up the following sectors of table 2.2 .

$$
I I B: \quad(N S+, N S+) \quad(R+, N S+) \quad(N S+, R+) \quad(R+, R+) .
$$

Summing all the states together we get the massless spectrum

$$
I I B: \quad[0]^{2}+[2]^{2}+[4]_{+}+(2)+\mathbf{8}^{2}+\mathbf{5 6}^{2},
$$

where the square brackets denote an antisymmetric tensor, the round brackets a symmetric one and the label + indicates that only half of the degrees of freedom of the 4 -form are independent. In the spectrum we find: the 0-, 2- and 4- form fields $C_{0}, C_{2}$ and $C_{4}$ know as the $R R$ forms, the symmetric 2-tensor which behaves as a graviton and the suffix $s$ refers to the string frame, the real scalar $\phi$ called dilaton and the 2-form in the NS-NS sector referred to as the $B$-field (or Kalb-Ramond field). The fermionic part of the action can be obtained acting with the supersymmetry charges. The fermionic states consist of two gravitinos with the same chirality and two Weyl fermions in the fundamental spinorial representation of $S O(8)$.

The low energy effective action for these degrees of freedom is a 10-dimensional $\mathrm{N}=2$ supergravity theory know as IIB sugra7. The bosonic part of the action is given by

$$
\begin{aligned}
S_{I I B} & =S_{N S}+S_{R}+S_{C S} \\
S_{N S} & =\frac{1}{2 \kappa_{10}^{2}} \int d^{10} x \sqrt{-g_{s}} e^{-2 \phi}\left(R_{s}+4 \partial \phi \partial \phi-\frac{1}{2}\left|H_{3}\right|^{2}\right), \\
S_{R} & =-\frac{1}{4 \kappa_{10}^{2}} \int d^{10} x \sqrt{-g_{s}}\left(\left|F_{1}\right|^{2}+\left|\widetilde{F}_{3}\right|^{2}+\frac{1}{2}\left|\widetilde{F}_{5}\right|^{2}\right), \\
S_{C S} & =-\frac{1}{4 \kappa_{10}^{2}} \int C_{4} \wedge F_{3} \wedge H_{3},
\end{aligned}
$$

where

$$
\begin{aligned}
\tilde{F}_{3} & \equiv F_{3}-C_{0} \wedge H_{3} \\
\tilde{F}_{5} & \equiv F_{5}+\frac{1}{2} B_{2} \wedge F_{3}-\frac{1}{2} C_{2} \wedge H_{3}
\end{aligned}
$$

and $\kappa_{10}$ is the 10-dimensional Newton constant.

A difficulty worth mentioning is associated with the 5 -form $\tilde{F}_{5}$. The IIB spectrum (2.7) requires that $\tilde{F}_{5}$ is self-dual, i.e. $\tilde{F}_{5}=* \tilde{F}_{5}^{8}$. Therefore upon 10-dimensional integration

\footnotetext{
${ }^{7}$ In fact there are only two sugra theories in 10 -dimensions with $\mathrm{N}=2$. The other one is IIA sugra that can be obtained starting with 11-dimensions which is unique if we want to avoid particles with spin higher than two. Then, dimensional reduction to 10-dimensions leads to IIA sugra. One of the differences is that while in IIB the two sets of supercharges have the same chirality, in IIA the chiralities are opposite.

${ }^{8}$ The Hodge dual of a $p$-form in D-dimensions, usually indicated with a star, is a $(D-p)$-form obtained contracting all the indices of the $p$-form with the totally antisymmetric Levi-Civita tensor $\epsilon$.
} 
$\left|\tilde{F}_{5}\right|^{2}=\tilde{F}_{5} \wedge * \tilde{F}_{5}=0$ and this term in the Lagrangian is identically zero. For all situations considered in the present work, this issue can be simply solved considering $\tilde{F}_{5}$ a normal 5 -form and imposing the self-duality condition only after the equations of motion are derived 9 .

There is a useful field redefinition that makes the $S L(2, \mathbb{R})$ symmetry of the effective action explicit and takes us to the Einstein frame ${ }^{10}$. Let us define

$$
S \equiv e^{-\phi}+i C_{0}, \quad G_{3} \equiv F_{3}-S H_{3}, \quad g_{M N} \equiv g_{s M N} e^{-\phi / 2} .
$$

Then the action becomes

$$
\begin{array}{r}
S_{I I B}=\frac{1}{2 \kappa_{10}^{2}} \int d^{10} x \sqrt{-g}\left\{R-\frac{\partial_{M} S \partial^{M} S}{2(\operatorname{Im} S)^{2}}-\frac{G_{3} \cdot \bar{G}_{3}}{12 \operatorname{Im} S}-\frac{\left|\widetilde{F}_{5}\right|^{2}}{4 \cdot 5 !}\right\} \\
+\frac{1}{8 i \kappa_{10}^{2}} \int \frac{C_{4} \wedge G_{3} \wedge \bar{G}_{3}}{\operatorname{Im} S},
\end{array}
$$

which is invariant under $S L(2, \mathbb{R})$ transformations acting as

$$
S \longrightarrow \frac{a S+b}{c S+d}, \quad G_{3} \longrightarrow \frac{G_{3}}{c S+d},
$$

and leaving $\tilde{F}_{5}$ and $g$ invariant.

\subsection{D-branes}

In this section we introduce D-branes and their effective action. This is an important ingredient for the string inflationary models that we will construct in chapter 4 and 5 because our inflaton candidate will be an open string mode, i.e. a D-brane excitation. Recently, the action describing these excitations has been intensively studied in the framework of cosmology and it will be at the center of the analysis of sections 5.5 .3 and 5.6 .

In type IIB there are other dynamical objects, non-perturbative in nature, that we have not yet discussed. They are extended objects charged under the RR fields known as Dirichlet-branes or D-branes for short. The name is justified by the fact that D-branes can be seen as the objects where the ends of open strings are attached; as for the violin string, the Dirichlet boundary condition

$$
\delta X^{\mu}(\tau, 0)=0
$$

\footnotetext{
${ }^{9}$ Self-dual forms can exist only in d-dimensions with $d=2 \bmod 4$ (with Minkowski metric) because in these cases $*^{2}=+1$. In $d=0 \bmod 4$, in particular in the interesting 4 -dimensional case, $*^{2}=-1$ and therefore one can impose the imaginary self-duality condition: $F_{d / 2}= \pm i F_{d / 2}$. The situation is exchanged if we have a Euclidean instead of Minkowski metric. We will encounter this in the next section where we will make use of imaginary self-dual 3-forms in a Euclidean 6-dimensional space.

${ }^{10}$ Actually only the $S L(2, \mathbb{Z})$ part of this group is a symmetry of the whole type IIB string theory.
} 
implies that the end of the string is fixed in the $\mu$ direction. As in type IIB we have RR 0-, 2- and 4- forms plus their Hodge duals, we expect 2-, 4-, 6-, 8- and 10-dimensional extended objects carrying the respective $\mathrm{RR}$ charge. It is customary to refer to D-branes using the number of spatial dimensions (the time dimension is implicit); therefore in type IIB we will have stable D1-, D3-, D5-, D7- and D9-branest1.

The small oscillations of D-branes are described by open strings attached to them. The action governing the dynamics of a single Dp-brane is, at leading order in the $\alpha^{\prime}$ expansion,

$$
\begin{aligned}
S & =S_{D B I}+S_{C S}, \\
S_{D B I} & =-T_{p} \int d \xi^{p+1} e^{-\phi} \sqrt{-\operatorname{det}\left(G_{a b}+B_{a b}+2 \pi \alpha^{\prime} F_{a b}\right)}, \\
S_{C S} & =i T_{p} \int_{\Sigma_{p+1}} e^{2 \pi \alpha^{\prime} F_{2}+B_{2}} \wedge \sum_{q} C_{q},
\end{aligned}
$$

where $\Sigma_{p+1}$ denotes the world volume of the Dp-brane. The first term is known as the Dirac Born Infeld (DBI) action. $T_{p}$ is the tension of the Dp-brane given by

$$
T_{p}=(2 \pi)^{-p} \alpha^{(-1-p) / 2} .
$$

$G_{a b}$ and $B_{a b}$ are the metric and B-field induced on $\Sigma_{p+1} ; F_{a b}$ is the field strength of the gauge field living on the brane. The DBI action arises after quantizing the action for the open strings ending on the brane; the excitations parallel to the brane give a world volume gauge theory while the perpendicular excitations describe the deformation of the world volume itself. Notice that, for $B=F=0, S_{D B I}$ reduces to the geometrical action.

The second term is the Cern Simons action. The exponential has to be understood in its power law expansion and $q$ runs over all the RR $\mathrm{q}$-forms. Clearly there are just a finite number of non-vanishing terms. For $F=B=0$ the only one is the integral of $C_{p+1}$ over the $p+1$-world volume of the Dp-brane. This term proves the above statement that D-branes carry $\mathrm{RR}$ charges. In the presence of world volume fluxes, i.e. $F \neq 0$, the D-brane can carry also lower RR charges.

\subsection{Flux compactifications}

In this section, after motivating the study of flux compactifications we give an explicit example, known as the GKP setup [8], in the framework of type IIB. We describe the 10dimensional theory around a non-trivial flux background and its low energy 4-dimensional effective action.

\footnotetext{
${ }^{11}$ Actually D9-branes, being spacetime-filling, require O9-planes due to tadpole cancellation. O-planes are non-dynamical, negative tension objects in string theory defined by the fixed points of an orientifold projection which is the gauging of a world-sheet, parity-reversal operation, possibly combined with some other symmetry. Close to an O-plane, which in the case of an O9-plane means everywhere in the spacetime, the physics is described by type I string theory.
} 
Our starting point is the action 2.15 plus other terms that might arise in presence of localized sources like D-branes or O-planes (non-dynamical, negative-tension objects discussed in the footnote 11). This is a 10-dimensional theory while all experiments performed until now indicate that we are living in a 4-dimensional world. On the other hand, it is possible that a higher dimensional theory looks lower dimensional below a certain energy scale. This is an old idea [9, 10] that dates back to Kaluza and Klein (KK); if the extradimensions are compact and smaller than a certain length $\Lambda^{-1}$, then by the Heisenberg indetermination principle we need energies higher that $\Lambda$ to probe them.

We will follow this idea and assume that six of the nine space dimensions are very small. We take the typical extra-dimension length to be $m_{K K}^{-1}$ where $m_{K K}$ is the Kaluza-Klein energy scale. After KK dimensional reduction we will obtain a 4-dimensional effective field theory which is valid at energies much lower than $m_{K K}$.

It has been soon realized that performing the KK reduction starting with a trivial background in 10-dimensions leads to a bunch of massless scalar fields in the 4-dimensional effective theory called moduli. These fields would transmit long range interactions, similar to gravity, which have not yet been observed in nature. In fact dedicated experiments, e.g. testing the equivalence principle, have put stringent bounds on a possible "fifth force" [11, 12.

A way out is to look at non-trivial 10-dimensional vacua where some or all the bosonic fields have a non-vanishing expectation value. In particular one can consider a background where some p-form field strength appearing in the action 2.15) has a non-vanishing integral, called flux, around some p-cycle $\Sigma$. If the cycle is topologically non-trivial then the flux can not be continuously/classically unwound. This procedure is known, in the string theory context, as flux compactification; in the present work we will adopt this method to obtain, starting from type IIB in 10-dimensions a phenomenologically interesting 4dimensional theory.

\subsubsection{The GKP setup}

In this subsection, we will review the type IIB flux compactification setup proposed by Giddings, Kachru and Polchinski (GKP) in [8]. We make an ansatz for the 10-dimensional metric as a warped metric

$$
d s_{10}^{2}=e^{2 A(y)} \eta_{\mu \nu} d x^{\mu} d x^{\nu}+e^{-2 A(y)} \tilde{g}_{m n} d y^{m} d y^{n},
$$

where we use $y^{m}$ to parameterize the 6-dimensional internal manifold, the capital indices $M, N, \ldots$ are running from 0 to 9 , the Greek ones $\mu, \nu, \ldots$ from 0 to 3 and the Latin ones $m, n, \ldots$ from 4 to 9 . The factor $e^{A(y)}$ can depend only on the internal coordinates (4 to 9 directions) if we do not want to break Poincaré invariance. For the same reason, the 5 -form should be of the type

$$
\tilde{F}_{5}=(1+*) d \alpha \wedge d x^{0} \wedge d x^{1} \wedge d x^{2} \wedge d x^{3},
$$


for some arbitrary function $\alpha$ of the internal coordinates, which makes explicit its selfduality. The Einstein's equations (EE's) are straightforwardly obtained calculating the Riemann tensor from the ansatz (2.22) and the energy momentum tensor from (2.15) and (2.18). Tracing the EE's we obtain a useful constraint

$$
\tilde{\nabla}^{2} e^{4 A}=e^{2 A} \frac{G_{m n p} \bar{G}^{m n p}}{12 \operatorname{Im} S}+e^{-6 A}\left[\partial^{m} \alpha \partial_{m} \alpha+\partial_{m} e^{4 A} \partial^{m} e^{4 A}\right]+\frac{\kappa_{10}^{2}}{2} e^{2 A}\left(T_{m}^{m}-T_{\mu}^{\mu}\right)^{l o c} .
$$

The Bianchi identity, or equivalently the equation of motion, for the $\tilde{F}_{5}$ form is

$$
d \tilde{F}_{5}=H_{3} \wedge F_{3}+2 \kappa_{10}^{2} T_{3} \rho_{3}
$$

where $T_{3}$ is the D3-brane tension and $\rho_{3}$ is the 6-dimensional D3-charge density, e.g. a delta function for spacetime-filling D3-branes. The integral of (2.25) gives the D3-charge tadpole cancellation condition

$$
\frac{1}{2 \kappa_{10}^{2} T_{3}} \int H_{3} \wedge F_{3}+Q^{l o c}=0 .
$$

In the presence of D3-branes, D7-branes and O3-planes the Bianchi identity plus the EE's determine the solution.

- The 3-form flux $G_{3}$ has to be imaginary self-dual:

$$
*_{6} G_{3}=i G_{3} \text {. }
$$

- The warp factor is determined by the 5 -form $\tilde{F}_{5}$

$$
e^{4 A}=\alpha .
$$

The dilaton is sourced by the D7-charge and obeys the equation

$$
\tilde{\nabla}^{2} S=\frac{(\tilde{\nabla} S)^{2}}{i \operatorname{Im} S}-\frac{4 \kappa_{10}^{2}(\operatorname{Im} S)^{2}}{\sqrt{-g}} \frac{\delta S_{D 7}}{\delta \bar{S}} .
$$

Let us now discuss supersymmetry. The KK-reduction over a Calabi-Yau threefold with non-trivial holonomy reduces the number of supersymmetry charges by a factor $1 / 4$, from $\mathrm{N}=2$ in 10-dimensions to $\mathrm{N}=2$ in 4-dimensions. If an orientifold projection, i.e. the gauging of a world-sheet parity-reversal operation, is performed, another half of the supersymmetries is broken leaving an $\mathrm{N}=1$ sugra theory in 4-dimensions. Imaginary self-dual 3-form fluxes can be of the $(2,1)$ or $(0,3)$ type. The former preserve $\mathrm{N}=1$ supersymmetry while the latter would completely break it.

The $\mathrm{N}=1$ sugra in 4-dimensions is determined by specifying a Kähler potential, a superpotential and a gauge kinetic function [13, 14]. As our focus is on moduli which are 
gauge neutral we will concentrate on the first two ingredients. Moduli produced by the KK reduction over a Calabi-Yau manifold are of two types, Kähler moduli and complex structure moduli. The former specify the sizes of compact submanifolds while the latter determine the shape of the Calabi-Yau manifold.

The 4-dimensional Kähler potential comes out to be

$$
\left.\kappa_{4}^{2} K=-2 \ln \mathcal{V}-\ln [S+\bar{S})\right]-\ln \left(-i \int \Omega \wedge \bar{\Omega}\right)
$$

where $\mathcal{V}$ is the volume of the Calabi-Yau manifold written in terms of the Kähler moduli and $\Omega$ is the holomorphic $(3,0)$ form that depends on the complex structure moduli. $\kappa_{4}^{2}$ is the 4-dimensional gravitational constant expressed in terms of Newton constant as $\kappa_{4}^{2}=8 \pi G_{N}$. The superpotential takes the form suggested by Gukov, Vafa and Witten (GVW) [15]

$$
W=\int \Omega \wedge G_{3}
$$

and is independent of the Kähler moduli. The resulting $N=1, d=4$ scalar potential is

$$
V_{F}=e^{\kappa_{4}^{2} K}\left(G^{\bar{I} J} D_{J} W \overline{D_{I} W}-3 \kappa_{4}^{2}|W|^{2}\right)
$$

where we use $G_{J \bar{I}}$ to denote the Kähler metric obtained from the second derivative of (2.30) and $G^{\bar{I} J}$ is its inverse. This potential can in principle fix the complex structure moduli and the dilaton [16, 17]. On the other hand the peculiar form of $K$ in (2.30) and the fact that $W$ in (2.31) is independent of the Kähler moduli leads to the so called no-scale structure (reviewed in appendix D.3), i.e. the fact that the Kähler moduli are completely flat directions.

\subsection{Towards de Sitter vacua in string theory}

As we saw in the last section, in the GKP setup, we can stabilize the complex structure moduli and the dilaton using fluxes. In addition, it is possible to have a non-trivial warp factor that has many interesting phenomenological applications as in the RandallSundrum models [18, 19]. On the other hand, we saw that the no-scale structure implies that the Kähler moduli are still massless at tree level. This property is not expected to hold at quantum level because there is no symmetry preventing perturbative and nonperturbative corrections to lift these directions.

The effects of corrections in a simple setup were investigated by Kachru, Kallosh, Linde and Trived (KKLT) in [20]. The Kähler potential is corrected both perturbatively and non-perturbatively. The superpotential on the contrary is protected by non-renormalizations 
theorems [13] and is corrected only non-perturbatively. There are several effects which are known to produce non-perturbative corrections to the superpotential. Of particular interest in the type IIB setup of GKP are Euclidean D3-branes (that we refer to as E3instantons), and gaugino condensation on a stack of D7-branes. The former can induce new terms in the superpotential only when they wrap particular 4-cycles; the necessary and sufficient conditions on the cycles for this to happen were found by Witten in [21] in the fluxless case while the case with fluxes is more involved. In the following, we will simply assume that these conditions are met for certain cycles $\Sigma_{i}$.

The superpotential then takes the form

$$
W=W_{0}+W_{\mathrm{np}}=W_{0}+\sum_{i} A_{i} e^{-\frac{2 \pi}{n} T_{i}}
$$

where $n=1$ in the case of E3-instantons or $n=\#_{D 7}$ in the case of gaugino condensation; with $W_{0}$ we indicate the GVW part of the superpotential 2.31 which does not depend on the Kähler moduli; $T_{i}$ are complexified Kähler moduli whose real part is the volume of the four cycle $\Sigma_{i}$ wrapped by the E3-instanton or by the spacetime-filling stack of D7-branes. Finally, the prefactor $A_{i}$ depends in general on complex structure moduli, the dilaton and open string moduli when they are present. We postpone a more detailed discussion of $A_{i}$ to section 4.2 and 5.3 .

$W_{\text {np }}$ clearly breaks the no-scale structure (see appendix D.3 so that a potential for the Kähler moduli $T_{i}$ is generated. For a generic Calabi-Yau manifold and a generic choice of fluxes, the moduli potential in the 4-dimensional effective action is a quite complicated function of $\mathcal{O}(100)$ variables. A brute force approach to find the minima of this potential is feasible only in some simple models; we will, on the other hand, adopt an alternative strategy, first proposed in [20].

When the masses of the complex structure moduli and the dilaton generated by the fluxes are hierarchically larger than the masses of the Kähler moduli, it is possible to perform a two step procedure [20]: first, one stabilizes the complex structure moduli and the dilaton using fluxes and ignoring $W_{\mathrm{np}}$; second, one integrates them out and studies the potential for the Kähler moduli in presence of $W_{\mathrm{np}}$. If there is a hierarchical separation between the two sets of moduli, the second step can not drastically change the stabilization achieved in the first step.

The two step procedure is not always legitimate (e.g. see [22]), but there are for sure several interesting cases in which it is perfectly allowed [23]. The large volume scenario (LVS), that we will study in chapter 6, is an example where there is a generic argument for the existence of a hierarchy between the moduli masses. In that setup, the Kähler moduli masses have a different scaling with the volume compared to the masses of the complex structures and the dilaton; the volume is then stabilized at an exponentially large value which ensures that the hierarchy between the two sets of moduli can be obtained parametrically. 


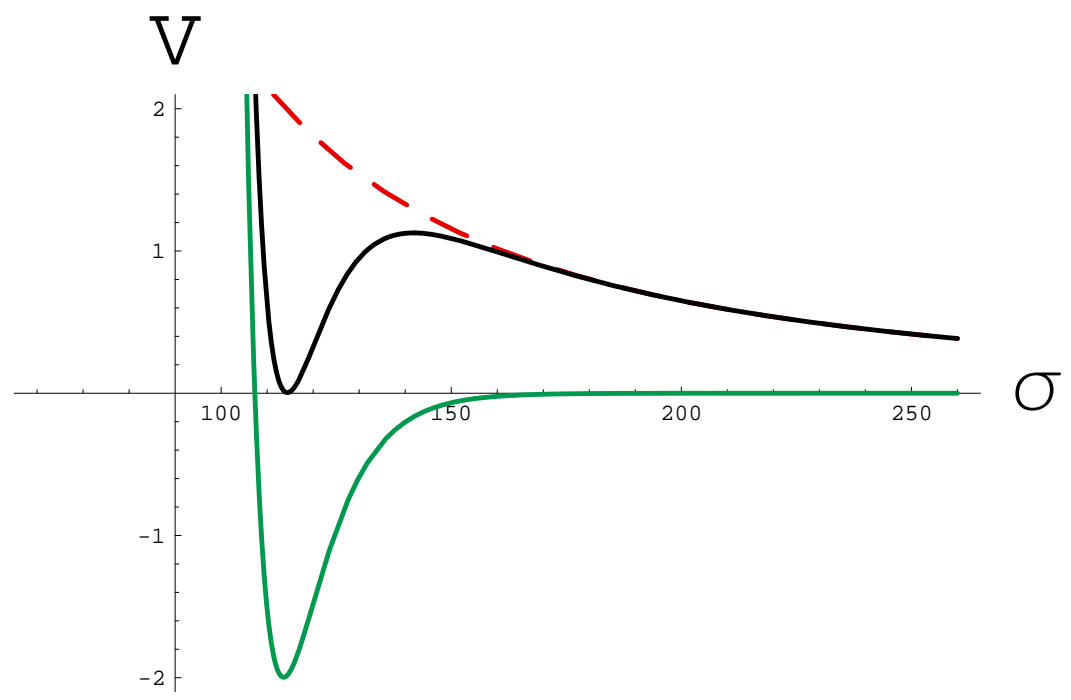

Figure 2.4: The AdS minimum is uplifted to a dS (or Minkowski) one; supersymmetry is broken and the minimum is metastable. Figure taken from [24].

In the sequel, we will therefore follow [20] and assume that the dilaton and the complex structures have been stabilized by fluxes and integrated out. The 4-dimensional $\mathrm{N}=1$ scalar potential 2.32 then becomes

$$
V=e^{\kappa_{4}^{2} K}\left(G^{\bar{J} I} D_{\bar{J}} \bar{W} D_{I} W-3 \kappa_{4}^{2}|W|^{2}\right)
$$

where $I, J$ run over the Kähler moduli only. For simplicity now we consider the case of a single Kähler modulus, the overall volume, which in terms of Hodge numbers means $h_{1,1}=1$. This is a technical assumption; if $h_{1,1}>1$, a potential is generically induced for all those $T_{i}$ for which a non-perturbative term in $W$ is present. The minimization of such a potential is clearly technically more involved.

The Kähler potential 2.30 in our case becomes

$$
\kappa_{4}^{2} K=-2 \ln (\mathcal{V})=-3 \ln (T+\bar{T})=-3 \ln (2 \tau),
$$

where $T=\tau+i b$, and $b$ is the axion that complexifies the overall volume Kähler modulus (see appendix D.3). Using the superpotential (2.33), we find

$$
V=\frac{a A e^{-a \tau}}{2 \tau^{2}}\left(\frac{1}{3} \tau a A e^{-a \tau}+W_{0}+A e^{-a \tau}\right)
$$

where the axion $b$ has already been minimized. The potential is the lowest curve of figure 2.4. The minimum is AdS and supersymmetric

$$
\begin{gathered}
D W=0 \longrightarrow W_{0}=-A e^{-a \tau}\left(1+\frac{2}{3} a \tau_{c r}\right), \\
V_{\text {min }}=-3 e^{K} W^{2}=-\frac{a^{2} A^{2} e^{-2 a \tau_{c r}}}{6 \tau_{c r}},
\end{gathered}
$$


where $\tau_{c r}$ is the value of the volume at the minimum. We can trust the supergravity approximation of string theory when the volume is large in string units. By (2.37) this implies that $\left|W_{0}\right|$, i.e. the value of the GVW superpotential after we integrate out the complex structure moduli and the dilaton, has to be much smaller than one. Typical values would for example be $A=1, a=0.1, W_{0}=10^{-4}$ and $\tau_{c r} \sim 110$. Although $\left|W_{0}\right|$ is typically of order one, given the huge number of possible flux configurations that we can choose, it is reasonable to expect that there will be some with $W_{0} \ll 1$.

To make contact with phenomenology there is still an important ingredient missing. For applications both to cosmology (chapter 4 and 5) and particle physics (chapter 6) we need a de Sitter or Minkowski vacuum. The idea [20] is to consider an uplifting mechanism that shifts up the AdS vacuum. Supersymmetry preserving vacua can only be AdS or Minkowski, therefore the uplifting will also break supersymmetry.

KKLT proposed an explicit construction to obtain an uplifting that introduces a parametrically small supersymmetry breaking. Suppose that in the compact manifold there is somewhere a warped throat (reviewed in appendix A) stabilized by imaginary self-dual fluxes as in GKP (section 2.5.1). Then any spacetime-filling D3-brane will preserve the same supersymmetries as the background; this BPS condition implies that the D3-brane will not feel any potential. On the other hand, an anti-D3-brane preserves a different set of supersymmetries than the background. This will break supersymmetry by an amount proportional to the anti-D3-tension $T_{3}$. Contrary to the D3-brane, the anti-D3-brane feels a non-trivial potential that pulls it towards the region of strong warping where its tension is redshifted. In the case of a warped throat, the anti D3 falls down the throat and stabilizes at the tip. Now the supersymmetry breaking term induced by the anti D3 is proportional to the warped tension. A direct calculation [25] leads to a term in the scalar potential

$$
V_{\text {up }}=\kappa_{4}^{2} \frac{2 h_{0}^{4} T_{3}}{g_{s}^{4} \tau^{2}} \equiv \frac{D}{4 \tau^{2}}
$$

where $h_{0}^{4}$ is the warp factor at the tip of the warped throat. Such a term induces a parametrically small susy-breaking in the sense that by an appropriate choice of fluxes the warp factor at the tip $h_{0}$ and therefore $V_{\text {up }}$ can be made arbitrarily small.

On the other hand, one can also require that 2.39 uplifts the AdS minimum to a dS or a Minkowski one. This is possible only for certain values of $D$ (which can in principle be obtained by a judicious choice of fluxes) and the amount of susy-breaking is no more arbitrary. Figure 2.4 shows how the $A d S$ minimum can be uplifted but not destroyed. By fine tuning $D$ one can actually obtain any value of the cosmological constant ${ }^{12}$,

\footnotetext{
${ }^{12}$ The choice of fluxes that determine $h_{0}$ and therefore $D$ are only discrete by charge quantization. On the other hand if many fluxes can be switched on, as it is the case for a generic Calabi-Yau manifold, then $D$ and therefore the cosmological constant can be fine tuned with a precision that scales exponentially with the number of fluxes. This issue was first addressed in the seminal paper [26] by Bousso and Polchinski; we refer the reader to it for further details.
} 
To summarize, we have found a vacuum in which all the moduli are stabilized, where supersymmetry is broken and there is a positive, in principle tunable cosmological constant. Although this vacuum is only metastable in the sense that it can tunnel to a decompactified Minkowski vacuum, its life-time can be made much larger than the age of the universe rendering this issue phenomenologically irrelevant.

The KKLT setup will be our starting point for more sophisticated constructions in chapters 4,5 and 6. Further comments on the stability of this construction under quantum corrections will be given at the beginning of chapter 6 . 


\section{Inflation in string theory}

The purpose of this chapter is to motivate the attempt to embed inflation in string theory. We start with a review of a series of recent cosmological observations that has changed, sometimes drastically, our picture of the universe. In particular, we review the results of measurements of the cosmic microwave background radiation (CMB for short). Interpreting these and other observations in the framework of general relativity has consolidated our knowledge about the big bang theory. Even more interestingly, from the point of view of high energy physics, the CMB has provided us with a precious tool to test speculations about the very early universe such as inflation.

We review and motivate the idea of inflation which is the most developed paradigm to understand the dynamics of the very early universe. Although inflation is a particularly simple mechanism and can explain astonishingly well all CMB measurements until now, it is still a speculation and more data are needed to establish it. Given this there has been a certain interest in constructing and developing alternatives to inflation. This is surely a very interesting research direction, for example because it is usually dangerous to interpret the data within a single paradigm. Nevertheless no alternative is, up to now, as developed and successful as inflation, which is why in the present work we have focused our attention on it.

We will give details about a particular class of single-field inflationary models (slow-roll models) which is both particularly simple and phenomenologically successful. We show how the CMB observables are related to the inflationary parameters. Having established inflation as an effective description we undertake the task of embedding it into full fledged string theory models. This task will occupy us in chapter 4 and 5 . Here we review some of the attempts present in the literature. We conclude the chapter with a discussion.

\subsection{Observations}

In this section we discuss some of the recent developments in observational cosmology with particular emphasis on the CMB measurements that provide precious information on the physics of the very early universe.

The last decade has been an extremely exciting and successful time for observational cosmology. Towards the end of the 90' several evidences were accumulated in favor of 

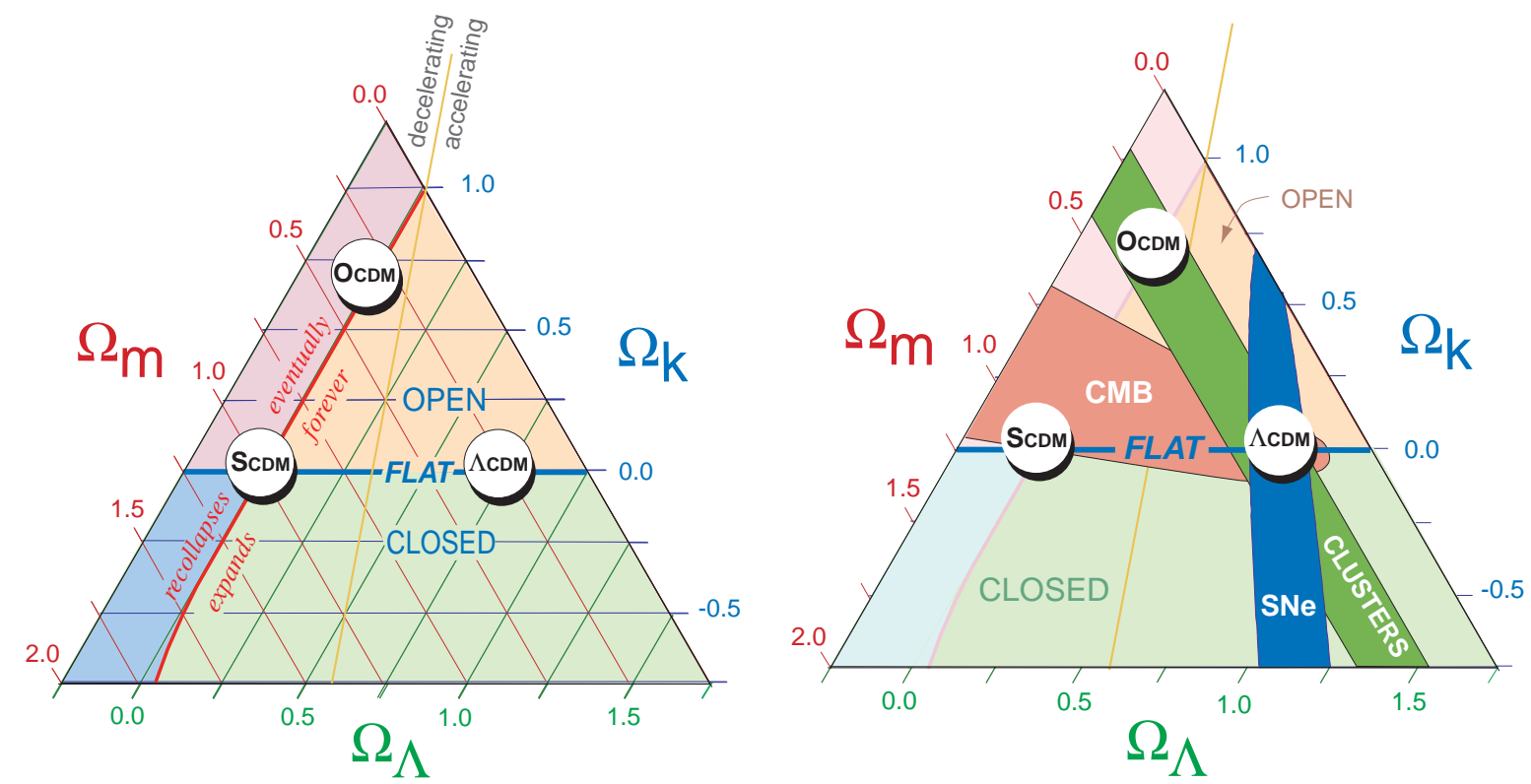

Figure 3.1: The three axes indicate the fraction of the critical energy density constituted by matter, dark energy and curvature, respectively. The left figure shows that the $\Lambda$ CDM model is the one consistent with CMB measurements, cluster surveys and supernovae data. Figure taken from [27].

the so called $\Lambda$ cold dark matter model $(\Lambda \mathrm{CDM})$, where $\Lambda$ indicates the cosmological constant. This simple model is consistent with a variety of different observations at different scales such as supernovae, galaxy rotation curves, cosmic microwave background radiation (CMB) and galaxy (cluster) surveys. An upshot of the observational results is given in figure 3.1. All data are compatible with a flat universe with critical total energy density, of which $72 \%$ behaves as dark energy, $23 \%$ as dark matter and the remaining $5 \%$ as baryonic matter. If the $\Lambda \mathrm{CDM}$ model correctly describes our world then we do not understand most of the energy of the universe.

Cosmological observations not only have astonishing implications for the present state of the universe but can teach us also a big deal about its evolution. In particular, CMB measurements [28, 29, 30] give us a snapshot of the universe at the time when photons decoupled and the universe became transparent, i.e. about $10^{5}$ years after the big bang. From this snapshot we can read out, among other things, the small perturbations that have lead to the formation of the large scale structures we observe.

The discovery of an extremely homogeneous microwave radiation at a temperature of 
about 2.7 Kelvin by Penzias and Wilson [31] provided a strong evidence in favor of the big bang model. Thirty years later the COBE experiment was able to detect the very small inhomogeneities in the CMB and estimated their intensity as one part in $10^{-5}[28$. What we see today in the sky within an angle of about 1 degree (multipoles $l<200$ ) corresponds to the size of the Hubble horizon at the moment of recombination, $10^{5}$ years after the big bang when the CMB was released. Hence we can divide inhomogeneities in large-scale $(l<200)$, which, because of causality, did not evolve so much after the primordial generation, and small-scale $(l>200)$ which are sensitive to all the physical processes inside the Hubble horizon. The former give us direct information about the universe much before recombination.

Precise data about the shape of the inhomogeneities have been collected by the WMAP experiment [29], which is still running and recently published the five-year data [30]. We briefly review some of the features of the CMB spectrum which are relevant for inflationary models and refer the reader to modern textbooks for further information [32, 33].

The CMB inhomogeneities can be decomposed into scalar, tensor and vector perturbations. Only the former have been detected; they appear to be Gaussian and adiabatid 1 . Their amplitude was first measured by COBE and can be expressed in terms of the power spectrum of the curvature perturbations $\mathcal{P}_{R}$. The measured value is approximatively $\mathcal{P}_{R} \sim 2 \cdot 10^{-9}$. The spectrum is very close to but not exactly scale invariant. The departure from scale invariance is parameterized in terms of the scalar spectral tilt $n_{s}$, defined such that $\mathcal{P}_{R} \propto k^{n_{s}-1}$. The WMAP five-year value is $n_{s}=0.96 \pm 0.014$ for negligible tensor modes (see figure 3.2, where $r$ is the ratio of the amplitude of tensor to scalar perturbations defined in (3.14)). As we will see, inflation provides a natural explanation for the smallness of $n_{s}-1$.

Vector perturbations are not expected to be present because they decay very fast. Tensor perturbations have not yet been observed and the current upper bound is $r<0.2$ (at $2-\sigma)$ [30]. Up to now the most developed and successful theoretical mechanism to understand the origin of the CMB perturbations is inflation, and we will review it in the next section.

\subsection{Inflation}

In this section we introduce the idea of inflation; for simplicity we focus on the single scalar field case. We describe the successes and the shortcomings of this mechanism.

General relativity (GR), governed by Einstein's Equations (EE's), is an extremely successful theory of gravity and it has passed all experimental tests up to now. Cosmological observations are interpreted in the framework of GR and provide us with a quite robust picture of most of the history of the universe. Unfortunately, the sharpness of the picture

\footnotetext{
${ }^{1}$ The search for non-Gaussianity is an active and promising research field. For a review see [34].
} 


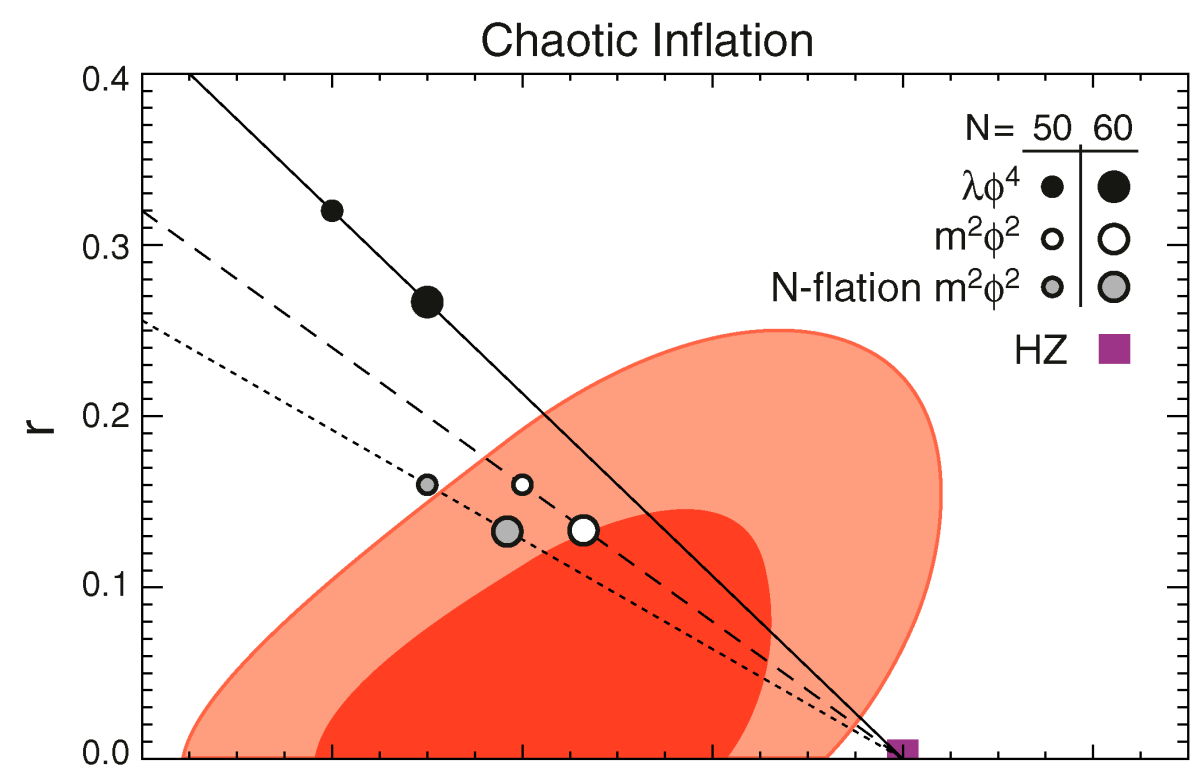

Figure 3.2: The five-year WMAP results in the $r-n_{s}$ plane, where $r$ is the tensor to scalar ratio and $n_{s}$ the scalar spectral tilt.

decreases exponentially as we approach the beginning of time. On scales much higher than $100 \mathrm{GeV}$ we do not even know which are the laws governing nature. Nevertheless, a series of problems with the standard big bang scenario led to the proposal [35, 36, 37, 38, 39] that some time between the Planck time and the electroweak epoch there has been a period of quasi exponential expansion of the universe. This phenomenon is known as inflation. Among the problems solved by inflation we have the flatness, the horizon and the monopole problems [32]. The former two are initial condition problems and they can be summarized saying that if the standard big bang scenario is extrapolated back to the beginning of time, then the initial values of the energy density and the curvature have to be extremely fine tuned.

Inflation can address these difficulties: if inflation starts, after a few Hubble times, the universe becomes very homogeneous, isotropic and flat. Hence we are allowed to consider a Friedmann Robertson Laimetre Walker ansatz for the metric of the form

$$
d s^{2}=d t^{2}-a^{2}(t) \delta_{i j} d x^{i} d x^{j}
$$

where $a(t)$ is the $t$-dependent scale factor. Formally, inflation means $\ddot{a}>0$, where the dots denote time derivatives. The EE's for the ansatz (3.1) reduce to the Friedmann equations:

$$
3 M_{P l}^{2} H^{2}=\rho, \quad M_{P l}^{2} \ddot{a}=-\frac{1}{6} a(\rho+3 p),
$$

where $H \equiv \dot{a} / a$ and we assumed that, whatever the matter content is, we can describe it using the hydrodynamical approximation with $\rho$ being the energy density and $p$ the 
pressure. It is clear from the second equation in (3.2) that inflation requires negative pressure: $p<-\rho / 3$.

There are several ways this can be achieved. One of the simplest and most widely studied possibilities is to assume that a canonically normalized scalar field $\phi$ with a potential $V(\phi)$ is the dominant source of energy. Then the equation of motion, energy density and pressure, in the case of a homogeneous $\phi$, are given by

$$
\ddot{\phi}+3 \dot{\phi} H=-V^{\prime}, \quad \rho=\frac{1}{2} \dot{\phi}^{2}+V, \quad p=\frac{1}{2} \dot{\phi}^{2}-V .
$$

When the potential energy is much larger than the kinetic one, the field's equation of state became $p=-\rho$, which mimics a cosmological constant. If $V$ was really constant, i.e. $\phi$-independent, the universe would be a de Sitter $(\mathrm{dS})$ space with $a \propto e^{H t}$. This would surely solve the initial condition problems but would also produce a totally empty universe. If eventually we want to make contact with the standard big bang scenario, inflation has to end and a thermal bath of Standard Model particles has to be produced hotter than some TeV. This implies that $\phi$ should roll down $V(\phi)$ and eventually settle in a minimum with a negligible cosmological constant. Oscillations around the minimum will then quantum mechanically decay, reheating the universe.

The duration of inflation is conveniently quantified in terms of the logarithmic growth of the scale factor

$$
N_{e} \equiv \ln \frac{a\left(t_{f}\right)}{a\left(t_{i}\right)},
$$

where $t_{i}$ and $t_{f}$ denote the time when inflation starts and ends, respectively, and $N_{e}$ is the number of e-foldings. For inflation to solve the initial condition problems we need $N_{e} \gtrsim 60$. For such a huge expansion to take place the scalar field has to roll slowly down the potential. A class of models where this can be achieved and the dynamics of the system is particularly simple are the slow-roll models that will be described in the next section.

A comment about the initial conditions is in order. For inflation to satisfactory solve the initial condition problems, one has to show that inflation itself can happen for generic initial conditions. Several efforts were performed in the early days of inflation to address this issue and they are reviewed in [40]. One of the issues is that the scalar field has to start far away from the minimum to ensure enough e-foldings. This led Linde [41] to study a class of potentials, where generic initial conditions lead to a prolonged stage of inflation; these are known as chaotic inflation models. Another problem is that inflation itself can actually start only in a relatively homogeneous and flat universe.

Addressing these and similar issues in cosmology require a measure. Unfortunately, a generically accepted measure for cosmology is not available and attempts to construct it often raise very deep issues in theoretical physics such as the arrow of time, the interpretation of quantum mechanics and the role of entropy in a theory of gravity just to mention 


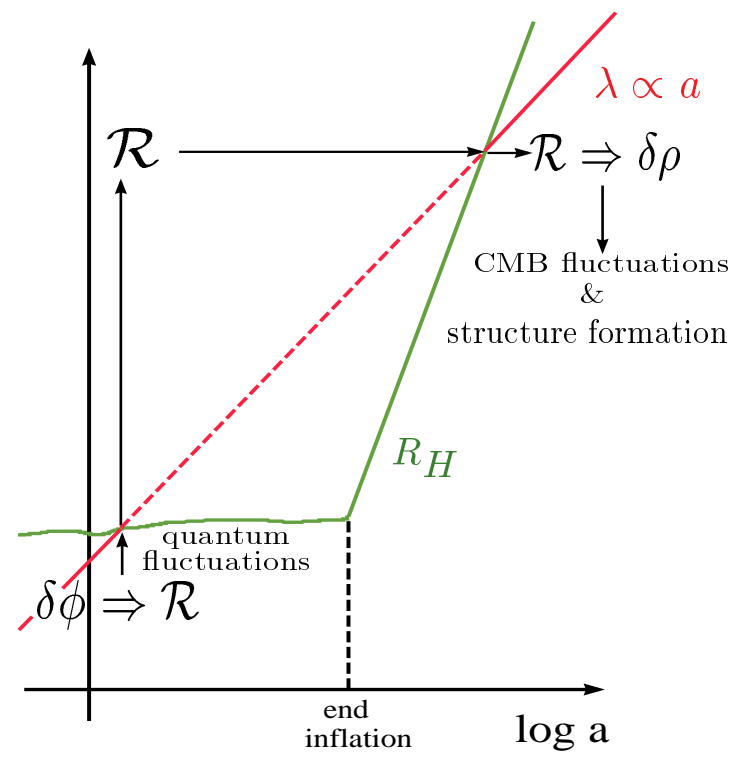

Figure 3.3: This is a cartoon of the generation of how the inflaton quantum fluctuations can generate the perturbations that we observe in the CMB. The figure is taken from [34].

a few. The state of the art is that it is largely acknowledged that inflation dramatically improves the initial condition problems (let aside the fact that it is a successfully predictive theory) but a better understanding of the pre-inflationary phase is needed to declare full success.

What has strongly increased the trust that inflation might be the right description of what happened in the early universe is the fact that, beyond improving the initial conditions problem for which it was proposed, this mechanism is extremely and unexpectedly successful in explaining the inhomogeneities that we observe in the CMB. The idea (see figure 3.3 is that the quantum fluctuations of the inflaton are stretched by the quasi exponential expansion. When their wave length is larger than the Hubble horizon they become frozen. After inflation is over, the universe enters a radiation and subsequently matter dominated phase during which the perturbations reenter the Hubble horizon. These inhomogeneities leave their imprint in the photons emitted at the end of recombination, who freely stream across the universe and arrive on the earth today in the form of the CMB.

\subsubsection{Slow-roll models}

In this section we show how to relate the CMB parameters to the inflationary physics and describe the constraints that arise for the various models. We will just state the results, 
referring to the textbooks [32, 33] for the derivation. In addition, we will concentrate on slow-roll models, a particular class of models which are easy to analyze and consistent with observations. In the next section and in section 5.5 .3 we will comment on alternatives.

A flat potential ensures that the field slowly rolls down and inflation lasts for a long time. Consider the following slow-roll conditions

$$
\begin{aligned}
\epsilon & \equiv 2 M_{P l}^{2}\left(\frac{H^{\prime}}{H}\right)^{2} \simeq \frac{M_{P l}^{2}}{2}\left(\frac{V^{\prime}}{V}\right)^{2} \ll 1, \\
\eta & \equiv 2 M_{P l}^{2} \frac{H^{\prime \prime}}{H} \simeq M_{P l}^{2} \frac{V^{\prime \prime}}{V} \ll 1
\end{aligned}
$$

where the approximations are valid up to higher order terms in the slow-roll parameters themselves. When these two conditions are fulfilled, the kinetic term in $\rho$ and the accelerations in (3.3) are negligible (slow-roll trajectory) and the equations simplify considerably. It can also be shown that, for slow-roll potentials, the slow-roll trajectory is an attractor in the field space, i.e. starting with arbitrary (homogeneous) $\phi_{0}$ and $\dot{\phi}_{0}$ the system reaches it within a few Hubble times. For slow-roll inflation the number of e-foldings is given by

$$
N_{e}=\ln \frac{a\left(t_{f}\right)}{a\left(t_{i}\right)}=\int_{t_{i}}^{t_{f}} H d t \simeq \int_{\phi_{f}}^{\phi_{i}} \frac{d \phi}{\sqrt{2 \epsilon}} .
$$

and can be quite larg $\oint^{2}$ because $\epsilon \ll 1$.

The scalar perturbations produced during slow-roll inflation are extremely close to Gaussianity which is in agreement with observations. For Gaussian perturbations, any n-point function with odd $\mathrm{n}$ is identically zero. This is the reason why the three-point (and higher odd) correlation function is a good tool to test the presence of non-Gaussianities. The detection of a non vanishing three-point function would rule out the simplest models, i.e. single-field slow-roll inflation. Although several tests have been performed, until now there is no convincing evidence of the presence of non-Gaussianities in the CMB spectrum. For further details we refer to the review [34].

The two important parameters describing scalar perturbations are the power spectrum $\mathcal{P}_{R}(k)$ of the comoving curvature perturbations $R$ and its spectral tilt $n_{s}$. They are given in terms of the slow-roll parameters as

$$
\begin{aligned}
\mathcal{P}_{R}(k) & =\frac{H^{2}}{2 \pi \dot{\phi}} \simeq \frac{1}{24 \pi^{2} M_{P l}^{4}} \frac{V}{\epsilon}, \\
n_{s}-1 & \equiv \frac{d \ln \mathcal{P}_{R}}{d \ln k} \simeq-6 \epsilon+2 \eta
\end{aligned}
$$

\footnotetext{
${ }^{2}$ Of course one has to take into account as well the range of variation of the inflaton $\phi_{f}-\phi_{i}$. This is also controlled by the slow-roll conditions (3.5), which tell us how fast the potential changes.
} 
As we said, observations require $\mathrm{f}^{3} \mathcal{P}_{R}\left(k_{\text {pivot }}\right) \sim 2 \cdot 10^{-9}$ for a certain pivot scale $k_{\text {pivot }} \simeq$ $7.5 a_{0} H_{0}$ (the subscript 0 refers to the present value). This implies that

$$
\frac{V^{1 / 4}}{\epsilon^{1 / 4}} \simeq 0.027 M_{P l} \simeq 6.6 \times 10^{16} \mathrm{GeV},
$$

where $V$ and $\epsilon$ have to be evaluated at the time when the relevant scales for the CMB exit the horizon. The number of e-foldings $N(k)$ of inflation remaining when the scale $k$ exits the Hubble horizon can be obtained from 33 .

$$
N(k)=62-\ln \frac{k}{a_{0} H_{0}}-\ln \frac{10^{16} \mathrm{GeV}}{V_{k}^{1 / 4}}+\ln \frac{V_{k}^{1 / 4}}{V_{\text {end }}^{1 / 4}}-\frac{1}{3} \ln \frac{V_{\mathrm{end}}^{1 / 4}}{\rho_{\text {reh }}^{1 / 4}},
$$

where the suffices "end" and "reh" refer to the end of inflation and recombination, respectively. Usually, the logarithmic terms are not expected to be too large, so one can say that the CMB perturbations exit the horizon about 60 e-foldings prior the end of inflation 4 .

As $\epsilon \ll 1$, the constraint 3.10 puts an upper bound on the scale of inflation (the energy density about 60 e-foldings before the end of inflation). For large field models $\left(\left|\phi_{i}-\phi_{f}\right| \gg M_{P l}\right)$ typically $\epsilon \lesssim \mathcal{O}\left(10^{-2}\right)$; hence inflation happens around the GUT scale. For small field models $\left(\left|\phi_{i}-\phi_{f}\right| \lesssim M_{P l}\right) \epsilon$ can be smaller by many orders of magnitude then the scale of inflation can be much lower (see e.g. section 5.5.2). Equation (3.9) incorporates a very successful prediction of slow-roll inflation: the spectrum is almost scale invariant. In actuality, WMAP five-years measured $n_{s}=0.96 \pm 0.014$ [30].

The analogous two parameters describing the tensor modes are

$$
\begin{aligned}
\mathcal{P}_{T} & =\frac{2}{M_{P l}}\left(\frac{H}{2 \pi}\right)^{2}, \\
n_{T} & \equiv \frac{d \ln \mathcal{P}_{T}}{d \ln k} \simeq-2 \epsilon .
\end{aligned}
$$

Notice that $n_{T}$ is defined differently from $n_{s}$. $\mathcal{P}_{T}$ does not depend on the slow-roll parameters because the tensor modes are generated exclusively by the perturbations of the metric (scalar perturbations can not generate tensor modes). The amplitude of tensor with respect to scalar modes is parameterized by the tensor to scalar ratio

$$
r \equiv \frac{\mathcal{P}_{T}}{\mathcal{P}_{R}} \simeq 12 \epsilon
$$

where $\mathcal{P}_{T}$ and $\mathcal{P}_{R}$ are approximated by their $k$-averaged scale-invariant value. Tensor modes have not yet been observed; the WMAP five-year upper bound is $r \leq 0.2$ [30]. A

\footnotetext{
${ }^{3}$ This condition is sometimes expressed in terms of $\delta_{H}^{2} \equiv \mathcal{P}_{R} 4 / 25 \simeq\left(1.9 \times 10^{-5}\right)^{2}$.

${ }^{4}$ Of course there are cases in which some of the logarithmic terms should be taken into account. We will see an example in section 5.5 .2
} 
detection of tensor modes would be a more direct evidence of the existence of gravitational waves (which have already been observed indirectly in the Hulse-Taylor binary system [42, 43]). In addition, in combination with the constraint (3.10), it would determine the scale of inflation.

\subsection{String theory models of inflation}

The success of the mechanism of inflation described in the last sections naturally raises a series of fundamental questions such as:

- Which field plays the role of the inflaton?

- Where does the inflaton potential come from?

- How does the inflaton couple to the Standard Model fields?

These and similar questions can be addressed only in the context of a fundamental theory. In this perspective inflation can be a bridge from very high-energy (everything in between collider energies and $M_{P l}$ ) physics to observations. As we said in chapter 2 , string theory provides a consistent UV completion of GR and it is therefore a valid candidate for a fundamental theory. In this section we will briefly review and catalog a series of attempts to embed inflation into string theory. For reviews see [44, 45, 46, 47, 48].

String models of inflation can be divided in two classes: open and closed string models, depending on wether the inflaton candidate is an open string mode (e.g. the position of a D-brane) or a closed string mode (e.g. the size of the compact manifold), respectively.

\subsubsection{Open string models}

In type IIB with D-branes the physics in the regions close to the brane is described by type I superstring theory, i.e. the massless spectrum contains open string excitations. The effective action in 10-dimensions for a D-brane is the DBI action (2.18). To preserve Lorenz invariance, the D-brane has to be spacetime-filling, i.e. it should extend in the $(3+1)$ large dimensions and wrap an arbitrary cycle in the six compact dimensions. The parameters describing the embedding in the compact dimensions produce scalar fields in the effective 4-dimensional theory. The easiest case to consider is a spacetime-filling D3-brane which is point-like in the compact directions. In 4-dimensions there are therefore 6 real scalar fields $Y(t, x)$ describing the position of the D3-brane. The effective 4-dimensional action for these fields is obtained from the DBI action of section 2.4. 
In [49] it was proposed that one or more of these scalars could play the role of the inflaton. Since then, constructing and studying models of brane inflation has been a very active research area. The two important ingredients for the dynamics of a scalar field are the kinetic term and the potential.

For concreteness and simplicity let us consider the case of a D3-brane. Its kinetic term in the DBI action can be expanded $5^{5}$ in a power series of $(\partial Y)^{2}$. For small velocities $5^{6}$ the higher terms are negligible and we are left with a canonical kinetic term. In the presence of a potential for the D3-brane satisfying the conditions (3.5), we reproduce then a slowroll model. This was a widespread approximation to study brane inflation until recently, when it has been shown [50, 51] that the whole DBI action as well leads to very interesting phenomenological features. DBI inflation is an example of a model with non-canonical kinetic term (but without higher derivatives) which had already been considered in the past [52, 53]. An interesting new feature is that string theory provides a high-energy justification for the choice of a particular effective action.

The inflaton potential can be generated applying some forces to the D3-brane. One possibility is the mutual Coulomb plus gravitational attraction between the D3 and an anti D3-brane. This setup has the attractive feature that when the distance between the branes is of order the string length, a tachyon develops and the branes annihilate. This process provides a natural graceful exit from inflation and an interesting mechanism for reheating; we will further comment on this in section 5.1. Unfortunately, the brane-antibrane potential is typically too steep to drive slow-roll inflation. A possible resolution of this problem will be described in chapters 4 and 5 , where two models of brane inflation are explicitly studied.

Alternatively to the attraction from an anti brane, another possibility is that some fluxes induce a potential for the D3-brane. This was studied e.g. in the D3/D7 brane models [54, 55] where the fluxes are on the world volume of the D7-brane. Again this setup provides a mechanism for a graceful exit and in addition cosmic (fundamental [56]) strings can be produced. Also non-perturbative effects can create a potential for the D3-brane [57] and we will thoroughly study a model of this type in chapter 5 .

\subsubsection{Closed string models}

In this class of models the inflaton candidate is a closed string excitation. In type IIB closed string massless moduli are always present if we compactify on a fluxless compact manifold. An example is the volume of a Calabi-Yau manifold, often referred to as the universal Kähler modulus or breathing mode. As we saw in section 2.5 it is possible to stabilize the moduli introducing fluxes (and non-perturbative effects as in the KKLT

\footnotetext{
${ }^{5}$ For a more detailed discussion and an example see section 5.5 .3

${ }^{6}$ The interesting background for cosmology is the homogeneous one $Y(t, x) \simeq Y(t)$, therefore we can neglect spatial derivatives
} 
setup). Now the idea is that if the potential generated is flat enough, before reaching the minimum a period of inflation will be induced.

The mechanism of graceful exit and reheating in this class of models is the standard one: once the minimum is reached the expansion stops, the inhomogeneities of the inflaton then grow, condense and finally decay into Standard Model particles.

An example in this class is Kähler moduli inflation [58] where the inflaton is the volume of some 4-cycle of the Calabi-Yau manifold. This is realized in the large volume scenario (LVS) that we will review in chapter 6. In other models, e.g. the racetrack models [59, 60, 61], the inflaton is the axion complexifying the Kähler modulus and the potential is generated by non-perturbative effects. Another proposal involving axions is N-flation 62] where a large number of them realize the assisted inflation mechanism 63, (further comments will be given in section 5.5).

\subsection{Discussion}

Let us finally comment on some features common to string inflationary models and discuss what we can learn from string cosmology.

The presence of many moduli in the 4-dimensional effective action is a very common feature in flux compactifications. Closed string moduli arise from complex structure and Kähler deformations of the compact space. For a typical Calabi-Yau manifold there are $\mathcal{O}(100)$ independent deformations. If D-branes are present, there will be additional open string moduli in 4-dimensions. Assuming that a stage of inflation is realized, the most natural expectation is a multifield inflationary dynamics, where many or all of the moduli evolve at the same time. Useless to say that this introduces enormous technical difficulties.

It is therefore a widespread technical assumption that it is possible to take just one dynamical field and keep all others at their stabilized values. This is a good assumption when e.g. one of the moduli is much lighter than all the others. The hierarchy guaranties that the heavy moduli relax quite fast to their minima while the inflaton candidate is just at the beginning of its inflationary trajectory. Already in the single-field case, embedding inflation into string theory is a hard task; therefore, we will not attempt here to look at more complicated situations. Apart from their simplicity, another motivation to focus our attention on single-field models is that they can successfully reproduce observations. A typical feature of multifield models, that distinguishes them from single-field ones, is that part of the perturbations are isocurvature as opposed to adiabatic [32, 33]. Up to now, CMB measurements are consistent with exclusively adiabatic perturbations.

Let us go back to the single-field case. As we reviewed in section 3.2.1, for slow-roll models all the relevant observables are determined by the two slow-roll parameters. In this sense the slow-roll approximation erases much of the information about the high energy physics 
responsible for inflation. Therefore, constructing a consistent model of slow-roll inflation in string theory does not provide an indication that string theory correctly describes the laws of nature. On the other hand discovering when and how inflation can take place in the framework of string theory gives us a useful tool to find regions of the landscape suitable to describe our universe. Also, slow-roll inflation is one of the simplest possible cases and it is natural to start with it and see which obstacles we are confronted with by the consistency of string theory. Once we learn how to overcome these obstacles, e.g. moduli stabilization or the $\eta$-problem, we can consider more complicated models.

An even more exciting possibility is that string theory will guide us to non-slow-roll models with distinctive signatures. An example is DBI inflation [50, 51] that we will review in section 5.5.3. The presence of higher order derivative terms typically induces large non-Gaussianities and makes the model in principle falsifiable.

Every phenomenological application of string theory, e.g. to cosmology (see chapter 4 and 5) or particle physics (section 6), is based on an effective action and it is therefore limited by the validity of the action itself. For example we look at the massless spectrum neglecting $\alpha^{\prime}$ excitations and we disregard the whole KK tower. Also, almost every analysis is performed at classical level neglecting loop corrections. The effects that all these corrections can induce in the tree level approximated analysis should be carefully considered. This is what we will do in section 6 for the soft terms produced by the LVS scenario. In the string inflationary literature, loop and $\alpha^{\prime}$ corrections are often neglected. The philosophy is that they can be considered in a second step, once a successful tree-level model has been found. This will be our attitude in chapters 4 and 5 . Eventually, one would like to ensure that none of the possible corrections spoils the phenomenological successes of the model. In this regard, we are still at the beginning of the path that leads to a bona fide string theory model of inflation. 


\section{Radial brane inflation}

The material presented in this chapter is mainly based on [64]. We investigate the possibility, for a concrete type IIB flux compactification setup, to obtain a phenomenologically successful model of brane inflation. We consider a D3-brane moving in a warped (deformed) conifold. The potential for this mobile D3-brane is generated by the attraction of an anti D3-brane at the tip of the conifold plus the effects induced by the stabilization of closed string moduli. We study the motion along the radial direction of the warped conifold. We show that, for fine tuned values of the parameters, this dynamics induces a prolonged stage of inflation and generates the correct CMB perturbations.

One of the purposes of this case study is to understand how the consistency of string theory constrains or obstructs the explicit construction of an inflationary model. Beyond constructing a successful model of slow-roll inflation! we will learn some generic facts about the dynamics of a D3-brane in the KKLT setup (reviewed in section 2.6). The study in this chapter will provide several motivations to consider alternative models of brane inflation. This will be widely discussed in chapter 5 where we propose and analyze a model of inflation at the tip of the conifold, where no anti D3-branes are present and the motion takes place in the angular directions.

The structure of this chapter is as follows: in section 4.1 we briefly review the state of the art about brane inflation and explain which improvements are provided by the present work; in section 4.2 we describe the structure of the effective superpotential and its relation to the D7-brane embedding. Section 4.3 explains the type IIB setup and reviews the $\eta$-problem for brane inflation, which forms a main issue in this chapter; the effective potential for the Kähler and open string moduli in the warped conifold background is obtained. In section 4.4 we perform the minimization of the potential in all but the radial directions for a generic D7-brane embedding. In section 5 we apply these formulae to two particular cases: the Ouyang and the Kuperstein embeddings. Only the latter, in a fine tuned case, gives rise to a prolonged stage of inflation. In section 4.6 we analyze how the stringy parameters determine the cosmological evolution and how they control when inflation takes place. A series of technical details are discussed in appendix B. In appendix B.5 we summarize the various forces acting on the D3- and anti D3-brane and comment on their relative importance.

\footnotetext{
${ }^{1} \mathrm{Up}$ to perturbative corrections that we do not take into account here. We postpone the discussion to chapter 6
} 


\subsection{Preliminaries}

One of the key steps to obtain a viable inflation scenario in string theory is to stabilize all massless moduli, except for the inflaton. As we reviewed in section 2.5 and 2.6, in the framework of type IIB flux compactifications, it is possible in principle to fix all closed string moduli.

If D-branes are present, an additional open string moduli sector is included. An interesting possibility (that we reviewed in 3.3) is to investigate if one of the open string moduli can play the role of the inflaton. If we have a pair of brane-anti brane, the Coulomb (plus gravitational) attraction could in principle drive inflation, where the distance between the branes would corresponds to the inflaton. For spacetime-filling D3-branes, this distance is just the separation along the compact directions. Clearly the distance between two points can not be larger than the size of the manifold itself. This geometrical bound on the range of variation of the inflaton prevents a prolonged stage of inflation. A way out was proposed in [65] (KKLMMT): if the branes are located in a region with strong warping, their reciprocal Coulomb attraction gets redshifted, the potential becomes flatter and inflation can last much longer.

Now, one has to ensure that no other forces spoil the achieved flatness of the Coulomb potential. Unfortunately, the stabilization of the Kähler moduli induces a force on the D3-brane. This is due to a non-trivial interplay between the volume and the D3-brane position. Once the Kähler moduli (among which the volume) are stabilized, the inflaton is endowed with a large mass. As a result, the second slow-roll parameter 3.5 turns out to be too large, $\eta \gtrsim 2 / 3$, showing the break-down of slow-roll inflation [65].

The idea of exactly canceling this moduli stabilization effect by fine tuning some other effect has received a certain amount of attention [57, 64, 66, 67, 68]. In this chapter, based on [64, 57, we show two ways in which this can be achieved: one is by using the uplifting term to cancel the inflaton mass and will be discussed in section 4.7 , the other is by means of threshold corrections to the non-perturbative superpotential. We will concentrate on the second possibility and construct explicitly a model where slow-roll inflation can take place.

Recently, threshold corrections to the non-perturbative superpotential $W_{\mathrm{np}}$, introduced in section 2.6, became available for the warped conifold background [69] (previously such effects had been calculated in [70] for toroidal orientifolds). This means, as we will describe in detail in section 4.2, that we know how the prefactor $A$ in $(2.33)$ depends on D3-brane open string moduli. We would like to use this effect to cancel the large mass induced by moduli stabilization (an example of the so called $\eta$-problem that will be review in 4.3). Unfortunately, this is not quite possible because the term we want to cancel is proportiona $\left.\right|^{2}$ to $\phi^{2}$ while threshold corrections arising from two large classes of

\footnotetext{
${ }^{2}$ Here we consider the D3-brane falling into the throat but still being far away from the tip. This allows
} 

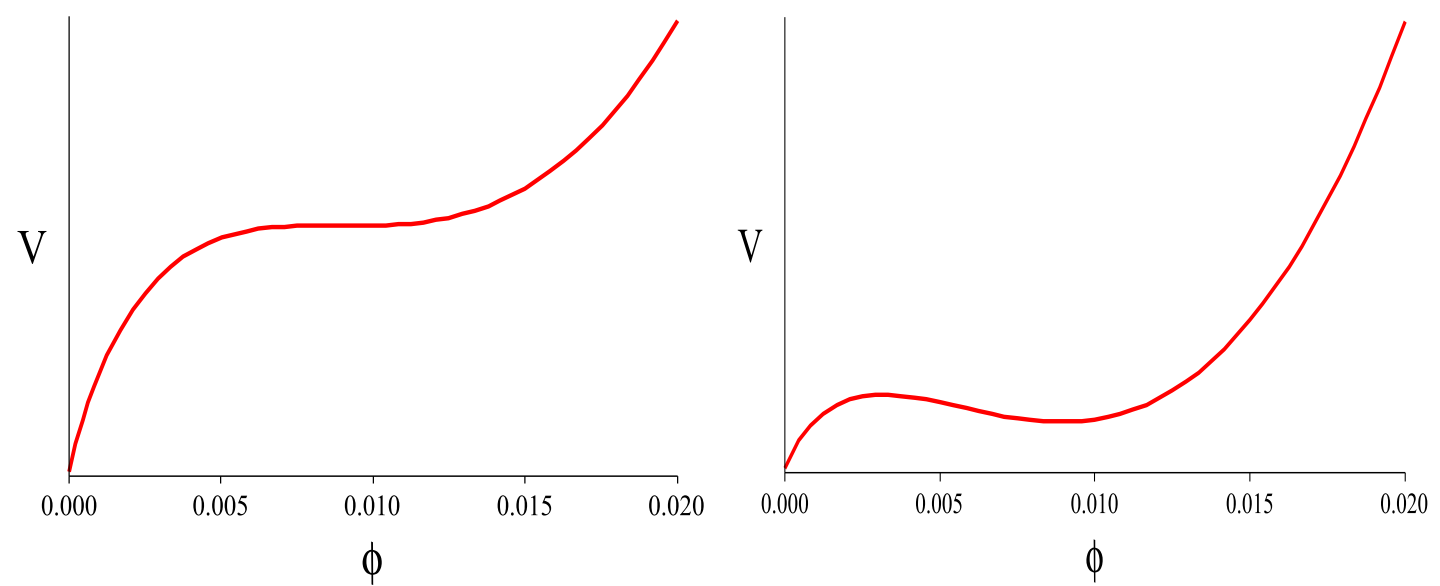

Figure 4.1: The plots display the inflaton potential $V(\phi)$ for the Kuperstein embedding for two different values of the uplifting parameter $\beta=1.21$ (left), $\beta=1.4$ (right). The left plot shows that via fine tuning an inflection point suitable for inflation can be obtained.

D7-embedding functions $g(w)$ (ACR [71, 72] and Kuperstein embeddings [73]) give rise only to terms proportional to $\phi$ and to integer powers of $\phi^{3 / 2}$; as we will see, this can be traced back to the holomorphicity of these supersymmetric embeddings. This is crucial because terms with a different $\phi$ dependence can cancel only locally in a small $\phi$ interval, rather than globally. Outside this small interval the inflaton potential is not of the slowroll type. In fact in general, the potential possesses a maximum and a minimum plus an inflection point in between, as shown in the right part of figure 4.1. With suitable finetuning, displayed in the left figure, it can be arranged that the maximum and minimum coincide with the inflection point, the potential hill at small $\phi$ disappears, and the potential becomes flat enough for slow-roll inflation.

We then study the cosmological evolution induced by the inflaton potential $V(\phi)$. In the fine tuned case in the left part of figure 4.1, a slow-roll inflation phase takes place (in particular $\eta \simeq 0$ ), but this happens only in a small region around the inflection point, where $\eta=0$ because the potential switches from concave to convex. We investigate when it is possible for the D3-brane to fall all the way into the throat and when the inflaton gets stuck somewhere in the throat. This is relevant both in the fine-tuned and in the more general case in which a maximum and a minimum are present. In fact, if one wants to end inflation with D3- anti D3-brane annihilation, the D3-brane has to go all the way down to the tip $(\phi \sim 0)$. If it gets stuck somewhere before, inflation lasts forever and the

us to approximate the deformed conifold with the singular conifold. Close to the tip, the effects of the deformation become relevant; we study this situation in appendix B.4. The result is that, close to the tip, the moduli stabilization produces a term proportional to $\phi^{3}$ which could be, contrary to the $\phi^{2}$ term for the singular conifold, cancelled by threshold corrections, at least in principle. Anyways, the cancellation can be achieved only for a short range of values of $\phi$ which is qualitatively analogous to the singular conifold situation. This is why we do not pursue this direction of investigation any further. 
universe becomes large and empty.

\subsection{The superpotential}

In this section, we continue the discussion of section 2.6 about the 4-dimensional superpotential generated by the dimensional reduction of type IIB flux compactifications. We will in particular focus on the non-perturbative corrections and their dependence on the open string moduli.

The GVW flux superpotential $W_{0}$ [15, 74], introduced in section 2.5.1, can fix the dilaton and the complex structure moduli. The Kähler moduli, on the other hand, are stabilized by non-perturbative effects [20] as we have seen in section 2.6. These effects break the no scale structure because of their explicit Kähler moduli dependence. Non-perturbative corrections to $W$, denoted by $W_{\mathrm{np}}$, can be generated either by Euclidean brane instantons, E3-instantons in our case, or by gaugino condensation on a stack of D7-branes. In the following we concentrate for concreteness on the latter possibility, but the result will be valid also for the former.

Suppose that a stack of $n$ D7-branes wraps a divisor $\Sigma$ defined by the zeros of a holomorphic function ${ }^{3} g(w)$. Gaugino condensation can generate a non perturbative term $W_{\text {np }}$ in the $N=1$ superpotential in addition to the GVW term $W_{0}$ [15]:

$$
W=W_{0}+W_{\mathrm{np}}=W_{0}+A e^{-a T},
$$

where $a=2 \pi / n$ with $n$ being the number of D7-branes (or $n=1$ for the Euclidean D3brane case). The prefactor $A$ appearing in $W_{\text {np }}$ depends in principle on the open string moduli, the complex structure moduli and the dilaton. In [76, 777, Ganor argued that $A$ has to depend on the open string moduli, that we indicate generically with $w$ here, in such a way that it vanishes when the D3-brane is on $\Sigma$. In [70], $A$ has been computed in the case of toroidal orientifolds. In [75, 69], it was shown how to generalize the calculation to curved space using the Green function method; this method allows one to determine only the dependence on the open string moduli. For the singular conifold and $Y^{(p, q)}$ cones the result [69] is

$$
A=A_{0} g(z)^{1 / n}
$$

where $A_{0}$ depends just on the complex structure moduli and the dilaton. Following KKLT [20], we will assume that these moduli have been stabilized by fluxes at a scale hierarchically higher than the scale of inflation (although this might not be the generic case, we are focussing our investigation on a corner of the landscape where this assumption holds).

\footnotetext{
${ }^{3}$ We use $g$ instead of $f$ as in 66, 69, 75, 64, 68, to avoid confusion with the DBI parameter, e.g. in (5.45).
} 
We are then allowed to integrate them out and focus on the dynamics of inflation; in the rest of the paper we will therefore treat $A_{0}$ as a constant. The consistency of 4.2 with Ganor's argument led to the conjecture that 4.2 is valid for a generic compactification [69].

From a different perspective, another argument in favor of (4.2) was formulated in [78 using the results of [79]. The idea of the argument is that, from the 10-dimensional point of view, the presence of non-perturbative corrections to the 4-dimensional superpotential means that the 10-dimensional Einstein equations do not require anymore an ordinary complex structure but a generalized complex structure [78. The latter determines then a non-trivial superpotential for the D3-brane (that is vanishing in the warped Calabi-Yau case). Using the potential of [79] and Ganor's argument [76, 77], one can obtain 4.2.

In addition, to simplify the analysis, we assume that the cycle $\Sigma$ is the only one up to continuous deformations. This means that we choose to work in a compactification with a single Kähler modulus. We denote its complexified field by $T=\tau+i b$. With an adequate shift of the axion $b=\operatorname{Im} T, A_{0}$ in $(4.2)$ can be taken to be real. The total superpotential is

$$
W=W_{0}+A(w) e^{-a T}=W_{0}+A_{0} g(w)^{1 / n} e^{-a T} .
$$

In this and the next chapter we will investigate the dynamics inside a warped conifold. In this region, the embedding of $\Sigma$ into the Calabi-Yau manifold is well approximated by an embedding into a (non-compact) conifold. There are two large classes of such embeddings on which we will focus our attention. The first one, found by Arean et al. in [71], contains the $A C R$ embeddings that can be written in terms of the $\left\{w_{i}\right\}$ coordinates of the conifold (see appendix A) as

$$
g(w) \equiv 1-\frac{\prod_{i=1}^{4} w_{i}^{p_{i}}}{\mu^{P}}=0
$$

where $p_{i} \in \mathbb{Z}, P \equiv \sum_{i=1}^{4} p_{i}$, and $\mu \in \mathbb{C}$ are (constant) parameters. The simplest choice $p_{i}=\delta_{1, i}$ reproduces the Ouyang embedding [72] which we will consider for the detailed study of section 4.5.1. The $p_{i}$ have to be integers by holomorphicity of supersymmetric embeddings was found by Kuperstein in [73]. This is conveniently written in terms of other conifold coordinates that we denote by $\left\{z_{i}\right\}$ (see appendix A). The Kuperstein embeddings are given, up to $S O(4)$ permutations, by

$$
g(z)=\tilde{g}\left(z_{2}^{2}+z_{3}^{2}\right)-z_{1}=\tilde{g}\left(z_{23}\right)-z_{1},
$$

where $\tilde{g}\left(z_{23}\right)$ is a holomorphic function of its argument $z_{23} \equiv z_{2}^{2}+z_{3}^{2}$. The $\left\{z_{i}\right\}$ are linear combinations of the $\left\{w_{i}\right\}$ (see appendix A), so again only integer powers of $\left\{w_{i}\right\}$ (equivalently $\left\{z_{i}\right\}$ ) are allowed by holomorphicity. This will play a crucial role in the following.

\footnotetext{
${ }^{4}$ In the Ouyang case, the integer $p_{1}=P$ can be interpreted as the number of times the D7-branes are wrapped around the 4-cycle.
} 


\subsection{Warped Brane inflation}

In this section, we review the $\eta$-problem arising for D3-brane inflation in a warped throat driven by brane-anti brane attraction, first pointed out in [65. Then we add threshold corrections to the analysis and obtain the F-term potential that we will study in the next section.

\subsubsection{The $\eta$-problem from volume stabilization}

It was pointed out in [65], that the strongest force felt by the D3-brane comes from the mixing of open string moduli with the overall volume, once the latter is stabilized à la KKLT. To see how this comes about, let us briefly review the result of section 2.6. Upon reducing the 10-dimensional type IIB over the warped metric background

$$
d s_{10}^{2}=h^{-1 / 2} d s_{4}^{2}+h^{1 / 2} d s_{6},
$$

where $h$ is the warp factor 5 , in the presence of imaginary self-dual fluxes and orientifold planes, we obtain a 4-dimensional, $N=1$ supergravity theory. The F-term potential for the Kähler moduli plus the anti D3-brane uplifting term were found to be

$$
\begin{aligned}
V_{\mathrm{dS}} & =V_{\mathrm{AdS}}+V_{\mathrm{up}} \\
& =\frac{a A_{0} e^{-a \tau}}{2 \tau^{2}}\left(\frac{1}{3} \tau a A_{0} e^{-a \tau}+A_{0} e^{-a \tau}+W_{0}\right)+\frac{D}{4 \tau_{c r}^{2}} .
\end{aligned}
$$

The values of $W_{0}$ (the GVW potential introduced in section 2.5.1) and $D$ (proportional to $h_{0}^{-1}$, the warp factor at the tip of the throat) depend on the fluxes which stabilize the complex structure moduli and the dilaton. Given the large freedom in the choice of fluxes, we will treat these quantities effectively as tunable constants. It is useful to re-express $\left\{W_{0}, D\right\}$ in terms of two other quantities $\left\{\tau_{0}, \beta\right\}$ as

$$
\begin{aligned}
W_{0} & =-A_{0} e^{-a \tau_{0}}\left(1+\frac{2}{3} \tau_{0} a\right), \\
D & =\beta \frac{2}{3} \tau_{0} a^{2}\left|A_{0}\right|^{2} e^{-2 a \tau_{0}} .
\end{aligned}
$$

The parameters $\left\{\tau_{0}, \beta\right\}$ have the following meaning: $\tau_{0}$ is the KKLT minimum, i.e. the value of $\tau$ in the AdS minimum obtain with $D=0$ (see section 2.6). Adding the uplifting $(D \neq 0)$, the minimum of (4.7) is shifted to $\tau=\tau_{c r}$ which is very close to $\tau_{0}$ (see appendix B.1.1); in fact $\tau_{c r}-\tau_{0} \equiv \Delta \ll \tau_{0}$. Hence, $\tau_{0}$ is an estimate of the position of the actual minimum and if it is chosen to be large enough we can neglect $\alpha^{\prime}$ corrections (see section

\footnotetext{
${ }^{5}$ Notice that we have changed the notation with respect to 2.22 to facilitate the comparison with the literature on brane inflation.
} 
6.2). As regards $\beta$, it parameterizes the uplifting in such a way that a Minkowski vacuum corresponds to $\beta \simeq 1+2 \Delta / \tau_{0}$, i.e. a value slightly larger than 1 while for $\beta \gtrsim 1+2 \Delta / \tau_{0}$ we have a dS vacuum.

Now let us add a D3-brane to the picture. The F-term potential (cf. (2.34) ) is then

$$
V_{F}=e^{\kappa_{4}^{2} K}\left(G^{\bar{I} J} D_{J} W \overline{D_{I} W}-3 \kappa_{4}^{2}|W|^{2}\right)
$$

where the indices $I, J$ run over the complex Kähler modulus $T$ and the complex coordinates $w=w_{i}$ with $i=1,2,3$ describing the position of the D3-brane (see appendix A). The superpotential is the one in (4.3) but for this subsection we neglect the threshold corrections, i.e. we treat $A$ as a constant. Notice that this implies $W_{i}=0$. In the next subsection we will consider a generic $A(w)$ and see which form the F-term potential takes in that case.

As concerns the Kähler potential, it is still equal to $-2 \ln \mathcal{V}$ as in 2.30 but now the volume gets shifted by a $w$-dependent quantity, i.e. it acquires a dependence on the position of the D3-brane. In [80] it was proposed that, for a single Kähler modulus (determining the overall volume) the Kähler potential is

$$
\kappa_{4}^{2} K=-2 \log (\mathcal{V})=-3 \log [T+\bar{T}-\gamma k(w, \bar{w})] \equiv-3 \log U,
$$

where $\gamma=\kappa_{4}^{2} \tau_{c r} T_{3} / 3$ is a constant (see appendix B.1 of [68]). The function $k(w, \bar{w})$ is the Kähler potential of the Calabi-Yau manifold evaluated at the position $w$ of the D3-brane. The metric of a compact Calabi-Yau threefold is not known, but as long as we are intersted in the dynamics inside a warped throat, we can approximate it with the conifold metric. Actually we have in mind a warped deformed conifold but for the regions far away from the tip of the cone the metrics coincide. In chapter 5 we will study the dynamics at the tip and we will use the full deformed conifold metric. The singular conifold has a Kähler potential $k(w, \bar{w})=r^{2}$, where $r$ is the radial direction of the conifold (see appendix $\mathrm{A}$ for more details). We study the motion of the brane along this radial direction, and $r$ will be our inflaton candidate.

The F-term 4.10) calculated with the Kähler potential (4.11) and the superpotential (4.3) with constant $A$ can be minimized in all directions but $r$. We then rewrite $V(r)$ in terms of a canonically normalized field $\phi=r \sqrt{T_{3}}$, the result is

$$
\begin{aligned}
V_{\mathrm{dS}} & =\frac{M_{P l}^{4}}{\left(\phi^{2}-6 M_{P l}^{2}\right)^{2}}\left(\frac{9 D}{\tau_{c r}^{2}}-\frac{6\left|A_{0}\right|^{2} a^{2} e^{-2 a \tau_{c r}}}{\tau_{c r}}\right) \\
& \equiv 3 H^{2} \frac{36 M_{P l}^{6}}{\left(\phi^{2}-6 M_{P l}^{2}\right)^{2}},
\end{aligned}
$$

where we have introduced the Hubble parameter $H$ for $\phi=0$ (and used the slow-roll approximation to neglect $\dot{\phi}^{2}$ ). The fields $r$ and $\phi$ have dimension of a length and a mass, 
respectively, while $\tau$ has been normalized to be dimensionless. The slow-roll parameter $\eta$ is then

$$
\begin{aligned}
\eta_{\mathbb{K} L M T} & =M_{P l}^{2} \frac{V_{\mathrm{dS}}^{\prime \prime}}{V_{\mathrm{dS}}}=M_{P l}^{2}\left[3 H^{2} \frac{144\left(6 M_{P l}^{2}+5 \phi^{2}\right)}{\left(\phi^{2}-6 M_{P l}^{2}\right)^{4}}\right]\left[3 H^{2} \frac{36}{\left(\phi^{2}-6 M_{P l}^{2}\right)^{2}}\right]^{-1} \\
& =\frac{4\left(6 M_{P l}^{2}+5 \phi^{2}\right)}{\left(\phi^{2}-6 M_{P l}^{2}\right)^{2}} M_{P l}^{2} .
\end{aligned}
$$

From its definition, $\phi$ is positive and smaller than $\sqrt{6} M_{P l}$. At this value in fact, the volume $\mathcal{V}$ in (4.11) becomes zero (assuming that the Kähler modulus $T$ has reached its minimum $T+\bar{T}=2 \tau_{c r}$ ) and the shifted Kähler potential becomes singular. Therefore $\eta_{K L M T}$ is always bigger than $2 / 3$ (the conformal value attained for $\phi=0$ [65]) and slow-roll inflation never takes place without threshold corrections.

\subsubsection{F-term potential for the conifold}

In this subsection we repeat the calculation of the last subsection but now taking into account the threshold corrections to the non-perturbative superpotential discussed in 4.2 , i.e. we allow for a generic $A(w)$.

The Kähler potential is again the one in (4.11); the resulting F-term potential for a generic $W$ takes the form (cf. [66])

$$
\begin{aligned}
V_{F} & =V_{\mathrm{KKLT}}+\Delta V \\
V_{\mathrm{KKLT}} & =\frac{\kappa_{4}^{2}}{3 U^{2}}\left[(T+\bar{T})\left|W_{T}\right|^{2}-3\left(\bar{W} W_{T}+\text { c.c. }\right)\right] \\
\Delta V & =\frac{\kappa_{4}^{2}}{3 U^{2}}\left[\frac{3}{2}\left(\bar{W}_{\bar{T}} \sum_{i} w_{i} W_{i}+\text { c.c. }\right)+\frac{1}{\gamma} k^{\bar{j} i} \bar{W}_{\bar{\jmath}} W_{i}\right],
\end{aligned}
$$

where $W_{T} \equiv \partial_{T} W, W_{i} \equiv \partial_{i} W$. Note that all terms of the type $K^{\bar{\jmath} i} W_{\bar{\jmath}} W K_{i}$ cancel out precisely. $V_{F}$ would vanish if the superpotential $W$ were independent of $T$ and $w_{i}$ because of the no-scale structure. But it is not and it is not. Indeed, using the superpotential in equation (4.3), where the non-perturbative term depends on both $T$ and $w_{i}$, one finds

$$
\begin{aligned}
V_{\mathrm{KKLT}} & =\frac{\kappa_{4}^{2}}{3 U^{2}}\left[\left[(T+\bar{T}) a^{2}+6 a\right]|A|^{2} e^{-a(T+\bar{T})}+3 a\left(\bar{W}_{0} A e^{-a T}+\text { c.c. }\right)\right] \\
\Delta V & =\frac{\kappa_{4}^{2}}{3 U^{2}}\left[-\frac{3}{2} a\left(\bar{A} \sum_{i} w_{i} A_{i}+\text { c.c. }\right)+\frac{1}{\gamma} k^{\bar{\jmath} i} \bar{A}_{\bar{\jmath}} A_{i}\right] e^{-a(T+\bar{T})},
\end{aligned}
$$

where $A_{i} \equiv \partial_{i} A$. The separation into two terms is due to the fact that $\Delta V$ is non-vanishing only when $A$ is a non-trivial function of the $w_{i}$. In the last subsection, where we assumed that $A$ is a constant, $\Delta V$ was absent. Note also that $V_{\text {KKLT }}$ is not the same as $V_{A d S}$ in 
4.7) of last subsection. It differs in two ways: first, in $V_{\text {KKLT }}$ there is also a dependence on the angular moduli through the non-constant $A\left(w_{i}\right)$; second, due to the backreaction of the mobile D3-brane the volume modulus has become $U=2 \tau-\gamma r^{2}$ rather than simply $2 \tau$ and has acquired a dependence on the D3-brane radial position.

Since we want to find out whether warped D3-brane inflation is possible in this setting we need to be in a de Sitter space. We therefore add, as we did in the last subsection, an uplifting term

$$
V_{\text {up }}=\frac{D}{U^{2}}
$$

to $V_{F}$. This term can be induced e.g. by the warped anti D3 tension [20]; this is not essential for our purposes and other upliftings, such as D-term or F-term upliftings [81, 82, 83. can be used as well; they could also differ in the $U$ scaling. For concreteness, in this chapter we focus on the uplifting (4.19) while in section 4.7 we will comment on alternative choices. Notice that, due to the backreaction of the mobile D3-brane, the $U$ (defined in (4.11)) in the denominator of (4.19) depends on the D3-brane radial position $r$. The uplifting breaks supersymmetry and lifts the vacuum to a dS one.

\subsection{Critical points of the potential}

Our eventual goal is to identify the inflaton with the mobile D3-brane radial position $r$ and to study whether its potential

$$
V=V_{\mathrm{KKLT}}+V_{\mathrm{up}}+\Delta V,
$$

can lead to viable inflation. To this end, we have to ensure that there is no steep runaway in some other directions in the moduli space. Therefore we analyze the stabilization of all moduli besides $r$, which comprise the volume modulus $\tau$, its axionic partner $b$ and the angular moduli $\theta_{1}, \theta_{2}, \phi_{1}, \phi_{2}, \psi$. As we want to restrict ourselves to the case of single-field inflation, we have to require that the D3-brane motion does not modify considerably the stabilization of the other fields. A convenient regime to consider is

$$
|g(r)|^{1 / n}-1 \ll 1
$$

so that the critical value of the volume modulus $\tau_{c r}$ changes only slightly during the inflationary dynamics (see 4.30 and the related discussion). Although the dependence of $\tau_{c r}$ on $\phi$ is mild (so that during the inflaton motion the minimization of $\tau$ is only slightly corrected), it is crucial to determine the shape of the effective potential for the inflaton $V(\phi)$ (see also [68, 67]). In figure 4.2, we compare the effective potential $V\left(\tau_{f i x}, \phi\right)$ for some fixed values $\tau_{f i x}$ of $\tau$, with the correct effective potential $V\left(\tau_{c r}(\phi), \phi\right)$. The sections at constant $\tau=\tau_{f i x}$ of the potential differ even qualitatively from the correct effective potential $V\left(\tau_{c r}(\phi), \phi\right)$. In the following we will assume that 4.21) is satisfied. 

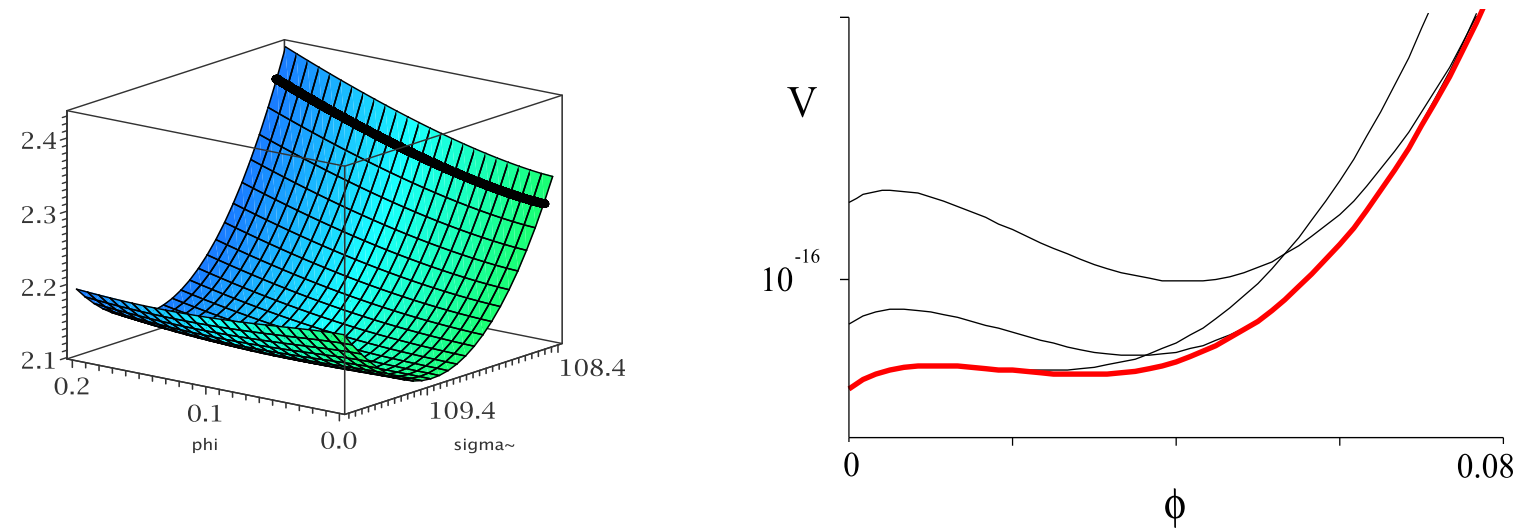

Figure 4.2: On the left: the dependence of the potential on $\phi$ and $\tau$ near the minimum. The black thick line is the value of $\tau_{c r}$ one would take neglecting the uplifting term (using just 4.27). Clearly if one is interested to inflation dynamics, neglect $V_{\text {up }}$ is inconsistent. On the right: the black thin lines are the potential evaluated for different but $\phi$ independent $\tau_{c r}$. The red thick one is obtained plotting $V\left(\phi, \tau_{c r}(\phi)\right)$. Again one clearly sees that it is inconsistent to study inflation just in the $\phi$ direction for fixed $\tau_{c r}$

\subsubsection{Axion stabilization}

It is easiest to start the moduli stabilization analysis with the axion field $b$. One observes that it makes its appearance only in the second term of $V_{\mathrm{KKLT}}$

$$
\begin{aligned}
3 a\left(\bar{W}_{0} A e^{-a(\tau+i b)}+\text { c.c. }\right) & =3 a\left|W_{0} A\right| e^{-a \tau}\left(e^{-i(a b-\alpha)}+e^{i(a b-\alpha)}\right) \\
& =6 a\left|W_{0} A\right| e^{-a \tau} \cos (a b-\alpha),
\end{aligned}
$$

where $\alpha$ denotes the phase of $\bar{W}_{0} A$. This term acquires its minimum when

$$
b_{c}=\frac{1}{a}[\alpha+(2 p-1) \pi], \quad p \in \mathbb{Z},
$$

and there it turns into minus its absolute value. This fixes the axion and implies for the KKLT part of the potential

$$
V_{\mathrm{KKLT}}=\frac{\kappa_{4}^{2}}{3 U^{2}}\left[2 a(a \tau+3)|A|^{2} e^{-2 a \tau}-6 a\left|W_{0} A\right| e^{-a \tau}\right] .
$$

\subsubsection{Volume stabilization}

This section is devoted to the minimization of the volume $\tau$, which is more involved than for the axion. The reason is that, as $r$ is our inflaton candidate, it is particularly important to determine the $r$ dependence of the critical value $\tau_{c}(r)$ of the modulus $\tau$. 
The criticality condition, $\partial_{\tau} V=0$, which determines $\tau_{c}$ can be expressed as

$$
\left(a U_{c}+2\right)\left(V_{\mathrm{KKLT}}+\Delta V\right)+2 V_{\mathrm{up}}=\frac{\kappa_{4}^{2} a^{2}}{3 U_{c}}|A| e^{-a \tau_{c}}\left(|A| e^{-a \tau_{c}}-3\left|W_{0}\right|\right),
$$

where $U_{c} \equiv 2 \tau_{c}-\gamma r^{2}$. If $\Delta V, V_{\text {up }}$ and the mobile D3-brane were absent, such that $U \rightarrow 2 \tau$, the criticality condition would give the original KKLT result [20]

$$
V_{\mathrm{KKLT}, 0}=-\frac{\kappa_{4}^{2} a^{2} A_{0}^{2} e^{-2 a \tau_{0}}}{6 \tau_{0}},
$$

with the KKLT critical volume $\tau_{c} \rightarrow \tau_{0}$ defined by

$$
W_{0}=-A_{0} e^{-a \tau_{0}}\left(\frac{2}{3} a \tau_{0}+1\right)
$$

where the fixed axion value (4.23) has been used. Once $V_{\text {up }}$ and the mobile D3-brane are added, the critical volume $\tau_{c}$ is moved away from $\tau_{0}$

$$
\tau_{0} \stackrel{V_{\mathrm{up}}, D 3}{\longrightarrow} \tau_{c} .
$$

Notice that $\tau_{c}$ depends on $D$ and $r$ while $\tau_{0}$ does not. We define

$$
\Delta(D, r)=\tau_{c}\left(W_{0}, D, r\right)-\tau_{0} .
$$

In what follows, we will use the parameters $\left\{\beta, \tau_{0}\right\}$ instead of $\left\{D, W_{0}\right\}$. We gave the definition in (4.8). As we said, the condition that $V_{\text {up }}$ uplifts the AdS minimum to dS is now easily expressed by the requirement $\beta \gtrsim 1+2 \Delta / \tau_{0}$ (which is very close to, but not exactly one). In the rest of the paper we assume that this condition is fulfilled and therefore the minimum is $\mathrm{dS}$.

Note that the full $r$ and $\beta$ dependence of $\tau_{c}$ is contained in $\Delta$. To calculate $\Delta$ we expand the criticality condition 4.25 in $\Delta / \tau_{0}$ and use $a \tau_{0} \gg 1$ to simplify the result. We obtain

$$
\partial_{\tau} V=0: \quad a \Delta\left(2|g|^{1 / n}-1\right)=\frac{\beta}{a \tau_{0}}|g|^{-1 / n}-\left(1-|g|^{1 / n}\right),
$$

where we keep the leading term and first subleading corrections in $1 / \tau_{0}$ and $\Delta / \tau_{0}$ of 4.25). This equation determines explicitly the $r$ dependence of $\Delta$, which arises due to the $\mathrm{r}$ dependence of $g$. Importantly from (4.30), taking into account (4.21) and that $a \tau_{0} \gg 1$, we can verify that $\Delta \ll \tau_{0} \sim \tau_{c r}$.

Without the D3-brane one would have $g=1$ and thus $\Delta=\beta / a^{2} \tau_{0}$ which in turn reduces to zero in the absence of the uplifting $(\beta=0)$ in agreement with the expectations. The consistency of our expansion is verified:

$$
\frac{\Delta}{\tau_{0}}=\mathcal{O}\left(\frac{1}{\tau_{0}^{2}}, \frac{|g|^{1 / n}-1}{\tau_{0}}\right) \ll 1 .
$$

Note that in general $\Delta$ depends, via the embedding $g$, also on the angular variables $\theta_{1}, \theta_{2}, \phi_{1}, \phi_{2}, \psi$ whose stabilization we are analyzing next. 


\subsubsection{Angular moduli stabilization}

For sake of brevity, let us denote the angular moduli

$$
\theta_{1}, \theta_{2}, \phi_{1}, \phi_{2}, \psi
$$

as $\vartheta_{\alpha}, \alpha=1, \ldots, 5$ and abbreviate $\partial_{\alpha} \equiv \partial_{\vartheta_{\alpha}}$. The criticality condition for the angular moduli, $\partial_{\alpha} V=\partial_{\alpha} V_{F}=0$, does not involve $V_{\text {up }}$ which is independent of $\vartheta_{\alpha}$. The full angular criticality condition thus reads

$$
\begin{aligned}
& 2\left(\left(2 a^{2} \tau_{c}+6 a\right)|A|-6 a\left|W_{0}\right| e^{a \tau_{c}}\right) \partial_{\alpha}|A| \\
= & \frac{3}{2} a \partial_{\alpha}\left(\bar{A} \sum_{i} w_{i} A_{i}+\text { c.c. }\right)-\frac{1}{\gamma} \partial_{\alpha}\left(k^{\bar{j} i} \bar{A} \bar{\jmath} A_{i}\right),
\end{aligned}
$$

where the lhs of the equality stems from $V_{\text {KKLT }}$ while the rhs originates from $\Delta V$.

As we did in the previous section, we replace $\tau_{c}$ by $\tau_{0}+\Delta$ and expand in $\Delta / \tau_{0} \ll 1$. Using (4.27) to evaluate the lhs of 4.33), one can see that the rhs of the criticality condition is suppressed by a factor $1 / \tau_{0}$ and thus does not contribute at leading order. One finds

$$
\tau_{0}\left(2-|g|^{1 / n}\right) \partial_{\alpha}|A|=0
$$

at leading order in $1 / \tau_{0}$ and $\Delta / \tau_{0}$. In view of (4.21) and (4.2), the values of the angular open string moduli that extremize the scalar potential are solutions of

$$
\partial_{\alpha} V=0: \quad \partial_{\alpha}|g|=0
$$

These five equations will generically fix all five angular moduli unless the embedding allows for isometries. However, isometries are incompatible with the bulk Calabi-Yau compactification and hence should be broken. For a detailed discussion of this issue see [75].

The fixing of the angular moduli can now be shown to lead to

$$
\Delta V=\frac{\kappa_{4}^{2}\left|A_{0}\right|^{2}}{12 n^{2} \gamma}|g|^{-2+2 / n} \partial_{r}|g|\left(-8 \pi \gamma r|g|+\partial_{r}|g|\right) \frac{e^{-2 a \tau_{c}}}{U_{c}^{2}} .
$$

Besides this, the other two contributions to the potential become

$$
\begin{aligned}
V_{\mathrm{KKLT}} & =\frac{2 \kappa_{4}^{2} a\left|A_{0}\right|^{2}}{3}|g|^{1 / n}\left(|g|^{1 / n}\left(a \tau_{c}+3\right)-\left(2 a \tau_{0}+3\right) e^{a \Delta}\right) \frac{e^{-2 a \tau_{c}}}{U_{c}^{2}}, \\
V_{\mathrm{up}} & =\frac{D}{U_{c}^{2}}
\end{aligned}
$$

where we have used (4.27) to eliminate $\left|W_{0}\right|$. We have thus achieved a stabilization of all moduli, except for $r$, the candidate inflaton. The dependence of the full potential on $r$ comes from the $r$ dependences of $\tau_{c}(r), \Delta(r)$ and $g(r)$. 


\subsubsection{Potential with fixed moduli}

We will now study in this section the potential in the large $\tau_{0}$ regime for a generic embedding $g$. For this we expand the potential in $\Delta / \tau_{0}$ and $1 / \tau_{0}$ and obtain at leading order

$$
\begin{aligned}
V_{\mathrm{KKLT}} & =V_{\mathrm{KKLT}, 0}|g|^{1 / n}\left(2-|g|^{1 / n}\right)\left[1+\frac{\gamma r^{2}}{\tau_{0}}\right] \\
\Delta V & =\frac{V_{\mathrm{KKLT}, 0}}{32 \pi^{2} \gamma \tau_{0}}|g|^{-2+2 / n} \partial_{r}|g|\left[8 \pi \gamma r|g|-\partial_{r}|g|\right] \\
V_{\mathrm{up}} & =\frac{D}{4 \tau_{0}^{2}}\left[1+\frac{\gamma r^{2}}{\tau_{0}}-\frac{2 \Delta}{\tau_{0}}\right] .
\end{aligned}
$$

In the expression for $V_{\text {KKLT }}$ there are also two terms proportional to $1-|g|^{1 / n}$ at order $\mathcal{O}\left(1 / \tau_{0}\right)$. Using (4.21), a posteriori justified in (4.30), we have omitted these terms. We see that $V_{\text {up }}$ appears suppressed compared to $V_{\mathrm{KKLT}}$ and $\Delta V$ by an additional factor $1 / \tau_{0}$ (after using 4.27) and 4.8).

Notice that in (4.39) we neglect the Coulomb (plus gravitational) attraction between the D3- and the anti D3-brane. As we explained in section 4.1, the reason is that the Coulomb attraction is very weak because of the warping. This was in fact the proposal of KKLMMT [65] to get slow-roll brane inflation. As we discussed in section 4.3, there are also effects coming from the F-term potential, which on the contrary is generically steep $\left(\eta_{\mathbb{K L M T}} \geq 2 / 3\right)$. Our effort is to make the F-term 4.39) slow-roll flat; once this is achieved, then we can add the Coulomb potential as well and study the resulting slow-roll inflation. Actually, motivated by this hierarchy of importance, we go even further: in chapter 5 we exclude any anti D3-brane to begin with, and just study the F-term, which is anyways always present.

Two assumptions are implicit in this result. The first is that $\tau$ reaches its $\phi$-dependent minimum (given by (4.30) ) instantaneously during the inflaton motion. In other words, the system evolves along the $\tau_{c r}(\phi)$ trajectory in the $\{\tau, \phi\}$ plane. For this assumption to be satisfied, the $\tau$ direction should always be much steeper than the $\phi$ direction which is actually the case here(see also the adiabatic approximation of [68]). The second assumption is more subtle and regards the angular directions. We are assuming that the initial conditions of inflation are such that these directions start at their minima. For the Ouyang and Kuperstein embedding that we consider in the next section, the minimum in the angular directions does not depend on the radial position. Therefore if, at the beginning of inflation, the system is at an angular minimum, it will stay there forever. This ad hoc assumption about the initial conditions is ubiquitous in the string inflationary literature. Anyways, it is more a technical than a conceptual issue: if the angular directions are steeper than the radial one, then even if they are excited at the beginning, they will relax in a short time; if they are flatter or comparably steep, then they should be included in the inflationary analysis which would become multi-field in nature. In 
the latter case one is obliged to rely on numerical methods loosing the intuition that the analytical single-field approach usually gives.

\subsection{Explicit examples: Ouyang vs Kuperstein embedding}

In this section we study two explicit supersymmetric D7-brane embeddings for the conifold. They have been discovered by Ouyang in [72] and by Kuperstein in [73]. We will find that for the Ouyang embedding $\Delta V$ vanishes at the minimum of the angular directions, where $\theta_{1}=\theta_{2}=0$. This was first noticed in [66]. As a result $\widetilde{\psi}$, defined by

$$
\widetilde{\psi}=\frac{1}{2}\left(\psi-\phi_{1}-\phi_{2}\right),
$$

remains unfixed. For the Kuperstein embedding, on the other hand, $\Delta V$ does not vanish at the minimum of the angular directions and can modify $\eta_{K K L T} \simeq 2 / 3$ (see also [68, 67]). It is worth noticing that, in the Ouyang case, if the maxima in the angular directions are inserted in $\Delta V$ then the resulting effective potential $V(\phi)$ is exactly the same as in the Kuperstein case. Of course this radial trajectory (that we will analyze in section 4.6) is physically interesting only in the Kuperstein case, where it is stable in the angular directions (an exhaustive analysis of this issue, with a detailed calculation has been given in [68]).

\subsubsection{Ouyang embedding}

The Ouyang embedding [72] is defined by the zeros of

$$
g\left(w_{1}\right)=1-\frac{w_{1}}{\mu} .
$$

Using A.6), one derives

$$
|g|^{2}=1-2 \frac{r^{3 / 2}}{|\mu|} \sin \frac{\theta_{1}}{2} \sin \frac{\theta_{2}}{2} \cos \widetilde{\psi}+\frac{r^{3}}{|\mu|^{2}} \sin ^{2} \frac{\theta_{1}}{2} \sin ^{2} \frac{\theta_{2}}{2} .
$$

We will take $\mu$ to be real and positive, as a possible phase can be absorbed in a shift of $\widetilde{\psi}$. The two directions perpendicular to $\widetilde{\psi}=$ const. are exactly flat at this point. They will eventually get a mass but their explicit value does not affect the effective potential for the inflaton.

The system of equations fixing the angles, $\partial_{\alpha}|g|=0$, turns into

$$
\begin{array}{rc}
\theta_{1}: & -\frac{r^{3 / 2}}{\mu} \cos \frac{\theta_{1}}{2} \sin \frac{\theta_{2}}{2} \cos \widetilde{\psi}+\frac{r^{3}}{\mu^{2}} \sin \frac{\theta_{1}}{2} \cos \frac{\theta_{1}}{2} \sin ^{2} \frac{\theta_{2}}{2}=0 \\
\theta_{2}: & -\frac{r^{3 / 2}}{\mu} \sin \frac{\theta_{1}}{2} \cos \frac{\theta_{2}}{2} \cos \widetilde{\psi}+\frac{r^{3}}{\mu^{2}} \sin ^{2} \frac{\theta_{1}}{2} \sin \frac{\theta_{2}}{2} \cos \frac{\theta_{2}}{2}=0 \\
\phi_{1}, \phi_{2}, \psi: & \frac{r^{3 / 2}}{\mu} \sin \frac{\theta_{1}}{2} \sin \frac{\theta_{2}}{2} \sin \widetilde{\psi}=0 .
\end{array}
$$


This system has two kinds of solutions (angular critical points)

$$
\begin{aligned}
& \theta_{1}=\theta_{2}=\pi, \quad \widetilde{\psi}=0, \pi \\
& \theta_{1}=\theta_{2}=0 \text { and } \widetilde{\psi} \text { unfixed. }
\end{aligned}
$$

A detailed study [66] (see also [68]) of the Hessian matrix shows that the solution corresponding to a minimum is $\theta_{1}=\theta_{2}=0$. Here we notice that the other angular direction $\widetilde{\psi}$ is not flat when $\theta_{i} \neq 0$. Once we evaluate the potential, however, at $\theta_{1}=\theta_{2}=0$, no dependence on $\widetilde{\psi}$ remains. The actual value of $\widetilde{\psi}$ does not affect the following result. In fact, we get

$$
\begin{aligned}
A & =A_{0} g^{1 / n}=A_{0}, \\
\Delta V & =0,
\end{aligned}
$$

so that the potential is exactly $V_{K K L T, 0}$, leading to $\eta \simeq 2 / 3$. In this case no fine tuning is possible [66]. The other extremum, $\theta_{1}=\theta_{2}=\pi$, corresponds to a maximum. In this case $\widetilde{\psi}$ is fixed (see $(4.46)$ ) but not the two perpendicular directions in $\left\{\phi_{1}, \phi_{2}, \psi\right\}$ space. If one substitutes these angular values (corresponding to a maximum), one obtains

$$
\begin{aligned}
A & =A_{0} g^{1 / n}=A_{0}\left(1+\frac{r^{3 / 2}}{\mu}\right)^{1 / n} \simeq A_{0}\left(1+\frac{r^{3 / 2}}{\mu n}\right), \\
\Delta V & =\frac{\kappa_{4}^{2}|A|^{2} e^{-2 a \tau}}{n^{2} R^{2}}\left[\frac{2 \pi r^{3 / 2}}{\sqrt{2} \mu+r^{3 / 2}}+\frac{r}{\gamma\left(\sqrt{2} \mu+r^{3 / 2}\right)^{2}}\right],
\end{aligned}
$$

where in the last step we have used (4.21), that translates into $r^{3 / 2} \ll \mu$ here and implies that the D3-brane is located further down in the throat than the D7-brane (which extends down to $r_{D 7}^{3 / 2}=\mu$ ). As we will see in the next section (see also [68]), the effective potential $V(\phi)$ that one obtains using this maximum (unstable in the angular directions) is exactly the same as the one for the Kuperstein embedding (4.52), where now the angular directions are at a minimum.

\subsubsection{Kuperstein embedding}

The simplest Kuperstein embedding [73] (that we will also consider in the chapter 5) is obtain from (4.5) with the choice $\tilde{g}\left(z_{23}\right)=\mu$. To facilitate the comparison with the Ouyang case, we factorize out from the Kuperstein embedding a factor $\mu$ and absorb it into the definition of $A_{0}$. Explicitly, we use the embedding

$$
g(z)=1-\frac{z_{1}}{\mu}
$$

where now we parameterize the conifold with alternative coordinates $\left\{z_{i}\right\}$ (the relation to $\left\{w_{i}\right\}$ is given in $(\mathrm{A} .3 \mathrm{D})$. This embedding has no directions along which $\Delta V=0$ [68, 67]. 
Two trajectories extremize the potential in the angular directions: $z_{1}= \pm r^{3 / 2} / \sqrt{2}$, but only the one with the negative sign is actually a minimum. The correction to the potential then becomes [68, 67]:

$$
\begin{aligned}
\Delta V & =\frac{\kappa_{4}^{2}|A|^{2} e^{-2 a \tau}}{n^{2} R^{2}}\left[-2 \pi \operatorname{Re} \frac{z_{1}}{\mu-z_{1}}+\frac{r}{\gamma\left|\mu-z_{1}\right|^{2}}\left(1-\frac{\left|z_{1}\right|^{2}}{2 r^{3}}\right)\right] \\
& =\frac{\kappa_{4}^{2}|A|^{2} e^{-2 a \tau}}{n^{2} R^{2}}\left[\frac{2 \pi r^{3 / 2}}{\sqrt{2} \mu+r^{3 / 2}}+\frac{r}{\gamma\left(\sqrt{2} \mu+r^{3 / 2}\right)^{2}}\right]
\end{aligned}
$$

which is exactly the same as in the Ouyang case after choosing the (in that case unstable) trajectory $w_{1}=-r^{3 / 2}$. The fact that the minus sign corresponds to the stable trajectory $\left(z_{1}=-r^{3 / 2} / \sqrt{2}\right)$ is crucial for the fine tuning of $\eta$. Indeed it determines that the correction to $\eta_{K K L T} \simeq 2 / 3$ comes with a minus and a cancellation is possible.

The potential we have written still depends on $\tau$. To obtain the effective potential for the inflaton we have to extremize the potential with respect to $\tau$, i.e. use 4.30 . The minimization of the volume can straightforwardly be carried out numerically. For an analytical estimate we will use (see appendix B.1)

$$
\tau_{c}=\tau_{0}+\frac{\beta}{a^{2} \tau_{0}}+\frac{r^{3 / 2}}{a n \mu}+\ldots,
$$

where the dots stand for terms suppressed by higher powers in $r^{3 / 2} / \mu$ or $1 / \tau_{0}$. We use this expression for the $r$-dependent critical value of $\tau$ to transform the potential $V(\tau, r)$ into a potential for a single field $V(r)=V\left(\tau_{c}(r), r\right)$. This implicitly assumes that the dynamics in the $\tau$ direction is much faster than in the $r$ direction such that the evolution of the system is well approximated by the trajectory $\tau_{c}(r)$ in the $(\tau, r)$ space. Eventually, the effective potential has to be expressed in terms of the canonically normalized field $\phi$.

\subsection{Inflation}

In the previous sections we calculated the potential for the radial position $r$ of the D3brane in the throat, once all other moduli have reached their minimum 6 . In this chapter we investigate if the potential we have obtained can provide phenomenologically viable inflation.

The first step is to rewrite the potential in terms of a canonically normalized field (to which we will refer in the following as the inflaton)

$$
\phi=\sqrt{T_{D 3}} r,
$$

\footnotetext{
${ }^{6}$ In section 4.5.2, we have not been specific about the moduli coming from the angular position of the D3-brane. As we said at the end of section 4.4.4, we assume that we start in a configuration where these moduli are already at their repective minima, from which values they do not move anymore.
} 
where we notice that $r$ has the dimension of a length while $\phi$ of a mass, as it should be for a canonically normalized scalar in 4-dimensions. We remember that $\tau_{0}$ is dimensionless and measures the four cycle volume in units of $l_{s}^{4}=\left(\alpha^{\prime}\right)^{2}$.

As we have seen in section 4.3 , $V_{\mathrm{KKLT}, 0}$ depends on the inflaton as 7

$$
V_{\mathrm{KKLT}, 0}=3 H^{2} \frac{36 M_{P l}^{6}}{\left(\phi^{2}-6 M_{P l}^{2}\right)^{2}} \simeq 3 H^{2} M_{P l}^{2}+H^{2} \phi^{2}+\ldots
$$

for small $\phi$. This prevents slow roll as

$$
\eta=M_{P l}^{2} \frac{V^{\prime \prime}}{V} \gtrsim \frac{2}{3}
$$

If we want to have a flat potential, we need another term in the potential of the same size but opposite sign that we can fine tune to cancel with the $2 / 3$. The new terms in the potential, coming from the dependence of the non-perturbative superpotential on $\phi$ as in (4.39), are proportional to $|g|^{1 / n}$ or to $\phi|g|^{1 / n}$. The known supersymmetric embeddings all depend on integer powers of $w_{i} \propto z_{i} \propto \phi^{3 / 2}$. This, in particular, implies that there is no term, in the small $\phi$ expansion, that can exactly cancel the $\phi^{2}$ from $V_{\mathrm{KKLT}, 0}$. The absence of fractional power embeddings, i.e. $g \propto w_{i}^{p}$ with $p$ non-integer, might be traced back to the holomorphicity of $g\left(w_{i}\right)$ (see also [67, 68]); it seems therefore hard to circumvent this problem.

Also, all those embeddings for which $g \propto 1+w_{i}^{p}$ with $p>1$ vanish much faster than $V_{\mathrm{KKLT}, 0}$ for $\phi \rightarrow 0$ and do not help to flatten the potential. From this observation, it follows that embeddings of the ACR family [71] with $p>1$ are not helpful to cancel the $\eta_{K K L M M T} \simeq 2 / 3$, at least for small $\phi$. Further study is needed to see if there is a region where $\phi$ is large enough so that the effects of higher ACR embeddings become relevant and at the same time, where that region is still well described by the conifold geometry (i.e. before the cut of the conifold and the gluing to the Calabi-Yau manifold become relevant).

Two embeddings that produce corrections to the scalar potential proportional to $\phi$ and $\phi^{3 / 2}$ (as opposed to $\phi^{p}$ with $p>2$ ) are the Ouyang (which is in the ACR family as well but with $p=1$ ) and the Kuperstein embedding. For the former, once the angular minimization is performed, the corrections to the scalar potential vanish [66]. For the latter this is not the case and the potential is indeed modified as in (4.52] [67, 68]. 


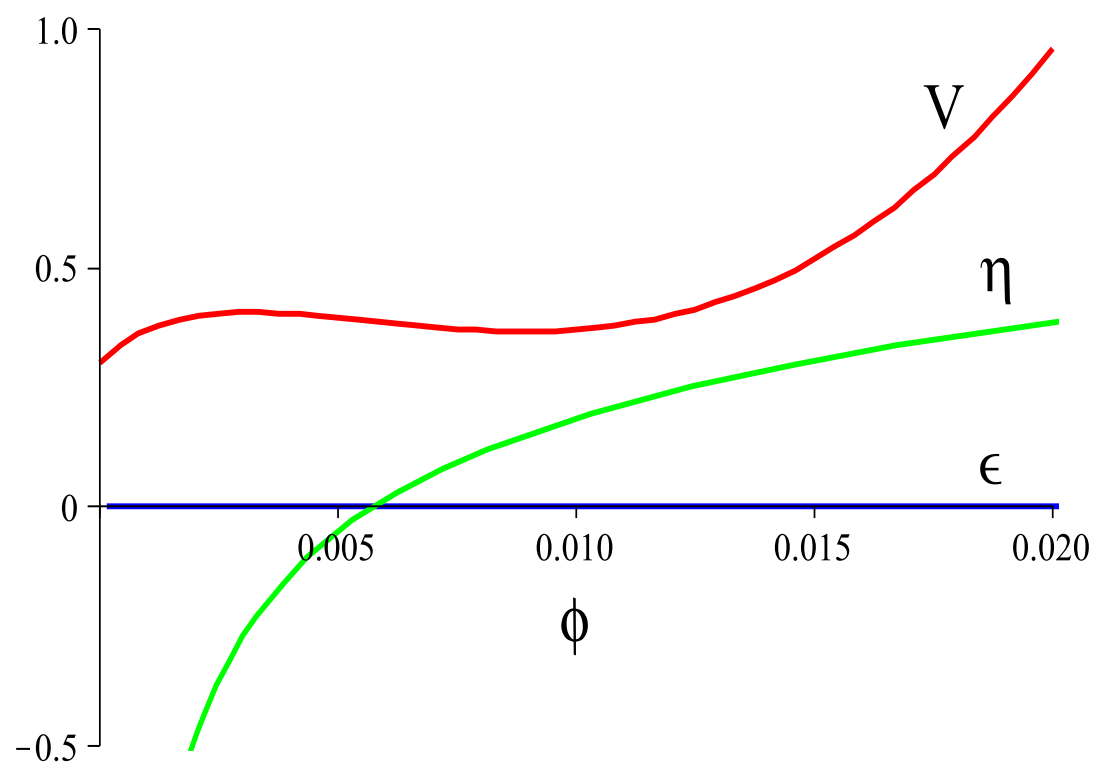

Figure 4.3: The plot shows the potential $V(\phi)$ (red) and the slow-roll parameters $\eta(\phi)$ (blue) and $\epsilon(\phi)$ (black). The latter is so small that it can hardly be distinguished from the $\phi$ axis. Next to the tip of the throat the potential has generically a maximum and a minimum. For $\phi$ large enough the potential grows like $\phi^{2}$ and $\eta$ is of order one (or bigger). But for $\phi \rightarrow 0$ the curvature of the potential changes at the inflection point and $\eta$ switches sign (and eventually diverges at $\phi=0)$.

\subsubsection{The effective inflaton potential}

Considering the region deep inside the throat, we expand the potential for small $r(\phi)$ keeping terms up to $r^{2}\left(\phi^{2}\right)$; higher terms can not cancel the $\eta_{K K L T} \simeq 2 / 3$ from $V_{K K L T, 0}$ anyway. The result is

$$
\begin{aligned}
V_{\mathrm{dS}} & \equiv V_{\mathrm{KKLT}}+V_{\mathrm{up}} \\
& =V_{\mathrm{dS}}^{(0)}+V_{\mathrm{dS}}^{(3 / 2)} \frac{r^{3 / 2}}{\mu n}+V_{\mathrm{dS}}^{(2)} \frac{\gamma r^{2}}{\tau_{c r}}+\ldots, \\
\Delta V & =\Delta V^{(1)} r+\Delta V^{(3 / 2)} \frac{r^{3 / 2}}{\mu n}+\ldots
\end{aligned}
$$

As shown in appendix B.2. $V_{\mathrm{dS}}^{(3 / 2)}+\Delta V^{(3 / 2)}<0$, so that the coefficient of $r^{3 / 2}$ is always negative. In terms of the canonically normalized field $\phi$, we want to study the effective

\footnotetext{
${ }^{7}$ We would like to stress again that this result is valid for the singular conifold which, far away from the tip, is a good approximation of the deformed conifold. In appendix B.4. we study the region close to the tip and find that the moduli stabilization procedure produces a term proportional to $\phi^{3}$ instead of $\phi^{2}$.
} 
Lagrangian

$$
\mathcal{L}=-\frac{1}{2} \partial_{\mu} \phi \partial^{\mu} \phi-\left(\Lambda+C_{1} \phi-C_{3 / 2} \phi^{3 / 2}+C_{2} \phi^{2}\right)
$$

where we have approximated the DBI kinetic term with the canonical one. A comment about this approximation is in order. As long as the kinetic energy of the inflaton $\dot{\phi}^{2}$ is small compared with the warped D3-brane tension, the higher terms in the expansion of the square root in the DBI action (2.18) are negligible (see subsection 5.5.3) and the canonical kinetic term gives a good approximation. In the present setup this condition is easily satisfied because inflation takes place in the middle of the throat where the warping is much weaker than at the tip. In chapter 5 we will study a model of inflation where the brane moves along the tip of a warped throat. In this model the effects of the DBI kinetic term become quickly relevant and we will thoroughly consider them in section 5.5.3.

The value of the cosmological constant term $\Lambda$ depends on several effective parameters. The stringy parameters are the 3-form fluxes in the conifold, which determine the stabilization of the complex structure and the dilaton. The problem of how the cosmological constant arises from string theory and which is its most probable value, is outside the scope of the present work (see e.g. the seminal paper [26]). In the following, we will simply consider $\Lambda$ as a free parameter. The coefficients $C_{1}, C_{3 / 2}$ and $C_{2}$ are such that the potential always has a maximum and a minimum (see appendix B.3); an extremal case is when these coincide and one gets a flat inflection point. In figure B.1 we show how varying $\beta$ (the uplifting parameter), the discriminant (see appendix B.3) can vanishes so that maximum and minimum coincide as in the left part of figure 4.1 .

In figure 4.3 , we plot $\eta$ together with the potential $V(\phi)$. The slow-roll parameter $\eta$ is small only in a narrow interval around a certain value $\phi_{\eta=0}$ which is where $V^{\prime \prime}$ (and therefore $\eta$ ) vanishes. For $\phi>\phi_{\eta=0}, \eta$ becomes of order one or bigger and is determined by $V_{\mathrm{KKLT}}$. For $\phi<\phi_{\eta=0}, \eta$ is instead determined by the correction $\Delta V$, it is negative and diverges as $\phi \rightarrow 0$ (but the potential can not be trusted all the way down to $\phi=0$ because of the deformation of the conifold at $r^{3 / 2}=\epsilon$ where the anti D3-brane sits and Coulomb and tachyon potentials become relevant). We want to stress that to get $\eta=0$ for some value of $\phi$ does not require any fine tuning. In fact $\eta$ is positive for large $\phi$ and negative for small $\phi$, so that for continuity it has to pass through zero.

A generic initial condition would be to start somewhere in the Calabi-Yau manifold and fall inside the throat. We therefore start at some $\phi_{\text {in }}$ and slide down towards smaller $\phi$. Qualitatively an important question is if one can reach the tip or if the D3-brane gets stuck somewhere before. Quantitatively a preliminary question is if one can get enough e-foldings and an almost scale invariant spectrum. 


\subsubsection{Inflation through an inflection point}

As we show in appendix B.3, the effective potential always has a maximum and a minimum. These coincide for a particular value of the uplifting $\beta$ giving rise to a flat inflection point at some $\bar{\phi}$. The critical $\beta$ can be estimated analytically from the zero of (B.21). In this section we comment on this fine tuned case (see also [68, 67] for an analysis of this potential). A crucial point is that around $\bar{\phi}$ the Coulomb potential (that we have argued could be neglected in the precedent discussion) has to be taken into account.

For example consider a $(\phi-\bar{\phi})^{3}+\Lambda$ potential, where the interesting case for us is $\Lambda \gg \phi^{3}$. The first derivative at the inflection point $\phi=\bar{\phi}$ is strictly vanishing. As a consequence the slow-roll attractor describes an inflaton that slows down exponentially and never reaches the inflection point. There are two effects that regularize this divergence: one are corrections to the strict slow-roll regime, such as an initial non-slow roll $\dot{\phi}$. This could allow to pass the inflection point in a finite time. A second effect (which co-exists with the first) are the subleading terms that we neglected in the potential; they can have a nonvanishing first derivative at the inflection point. An example is the generally subleading Coulomb potential, that becomes important around $\bar{\phi}$ (where the potential is otherwise flat). Linearly approximating $V_{D 3 \overline{D 3}}$ one gets a potential $(\phi-\bar{\phi})+(\phi-\bar{\phi})^{3}+\Lambda$. In this case, the inflection point is always reached and overshot.

The number of e-foldings $N_{e}$ that results from the inflationary dynamics is therefore controlled by the value of the first derivative of the potential at $\bar{\phi}, V^{\prime}(\bar{\phi})$. Varying $V^{\prime}(\bar{\phi})$ continuously, from positive to negative values, corresponds to a change of $N_{e}$ from just a few to infinity. An analytical estimate (neglecting for the moment corrections to slow roll) gives $N_{e} \propto 1 / V^{\prime}(\bar{\phi})$ for positive $V^{\prime}(\bar{\phi})$; for negative $V^{\prime}(\bar{\phi})$ a minimum of $V$ is formed and the issue of overshooting and slow-roll corrections becomes important. In any case, it is clear that an arbitrary large amount of inflation can be obtained by the potential we have calculated, provided that one can fine tune the string theory parameters to obtain a small $V^{\prime}(\bar{\phi})$. Taking into account the effects of the DBI action can only increase the number of e-foldings. In the next two sections we will go beyond the slow roll approximation and address the question, when does the D3-brane reach the tip and when does it get stuck somewhere in the middle?

A final comment is in order. In the present model the shape of the potential is determined by the F-term, while the Coulomb potential gives only relatively small corrections. These corrections are relevant only in the fine tuned case of a flat inflection point. Even in this case, the Coulomb potential dominates only around the inflection point where the force exerted by the F-term is vanishing. We want to contrast this situation with another expectation which is often found in the phenomenological brane inflationary literature (see e.g. [84, 85, 86]). One could have wished to find a way to completely get rid of the F-term effects and have an inflationary model based on a Coulomb potential of the type

$$
V \sim V_{0}\left(1-\frac{1}{\phi^{4}}\right)
$$


which have been widely studied (see e.g. [87, 84, 85, 86]). The result of the present investigation is that this is even harder to achieve that expected. For the class of embeddings we studied, not even fine tuning allows us to cancel the F-term effects. We consider this as an indication that in a generic model of brane inflation, the Coulomb attraction is superfluous because the inflaton potential is determined by the F-term potential. This observation is a strong motivation for the model we will propose in chapter 5 .

\subsubsection{Damped oscillatory phase}

In this and the next section we study the problem of overshooting. We make the following simplifying assumptions: we consider a homogeneous and isotropic universe so that the 4-dimensional Einstein equations reduce to the Friedmann equations (3.2); furthermore we assume that all fields have been stabilized except for the inflation; finally we neglect effects of the DBI action and approximate it by a canonical kinetic term as discussed around 4.58.

Let us start considering the following potential

$$
V=\Lambda+\frac{1}{2} m^{2}\left(\phi-\phi_{\min }\right)^{2}
$$

The first Friedmann equation gives

$$
H^{2}=\frac{1}{3 M_{P l}^{2}}\left[\Lambda+\frac{1}{2} m^{2}\left(\phi-\phi_{\min }\right)^{2}+\frac{1}{2} \dot{\phi}^{2}\right] \simeq \frac{\Lambda}{3 M_{P l}^{2}} .
$$

Therefore, the equation of motion can be approximated as

$$
\ddot{\phi}+\frac{\sqrt{3 \Lambda}}{M_{P l}} \dot{\phi}+m^{2}\left(\phi-\phi_{\min }\right)=0,
$$

where $\Lambda \gg m^{2}\left(\phi-\phi_{\min }\right)^{2}$. can be achieved with an appropriate choice of $\Lambda$ and $m$; the consistency of neglecting $\dot{\phi}^{2}$ in (4.61) will be checked at the end.

The equation of motion is the one for a harmonic oscillator with friction. There are three types of solutions:

- Underdamped: $M_{P l}^{2} m^{2}>3 \Lambda / 4$; this is the only case where the field actually oscillates around the minimum. The amplitude is exponentially decreasing with a typical time $\sqrt{3 \Lambda} / 2 M_{P l}$. If the field starts at $\phi_{i}$ at $t=0$ with $\dot{\phi}_{i}=0$, we can estimate the speed when it passes the first time through the minimum at $t=t_{\min }$ as

$$
\dot{\phi}_{\min }=\phi_{0} e^{-\sqrt{3 \Lambda} t_{\min } / 2 M_{P l}}\left[\frac{\sqrt{3 \Lambda}}{M_{P l}}-\frac{2 m^{2} M_{P l}}{\sqrt{3 \Lambda}}\right] \cos \left(t_{\min } \omega\right),
$$

where $\omega^{2}=m^{2}-3 \Lambda / 4 M_{P l}^{2}$, and $\phi_{0} \equiv \phi_{i}-\phi_{\text {min }}$ is the distance from the starting point to the minimum (see figure 4.4). 


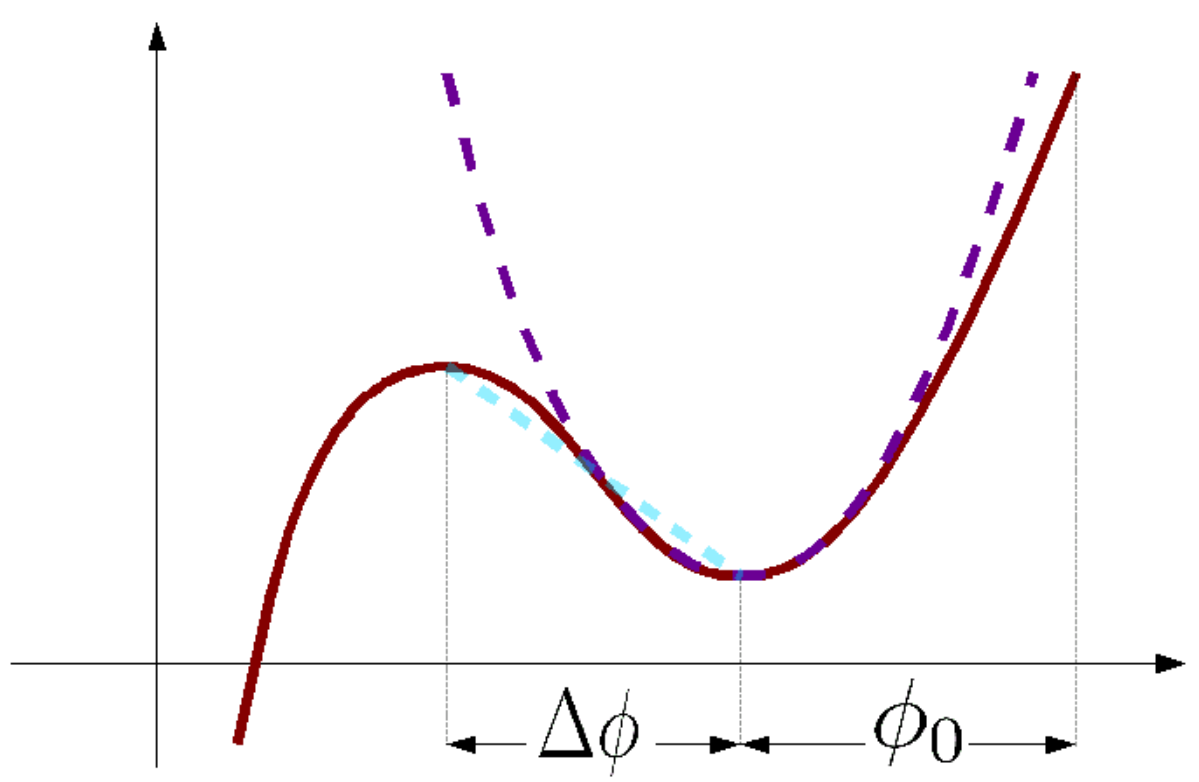

Figure 4.4: The figure summarises our overshoot analysis. The continuos line is the actual potential, the darker dashed line refers to the discussion of the damped oscillatory phase and the lighter dashed line refers to the uphill phase.

- Critically damped and overdamped: $M_{P l}^{2} m^{2} \leqslant 3 \Lambda / 4$. There are no oscillations and the field takes an infinite amount of time to reach the minimum (where $\dot{\phi}=0$ ).

The underdamping condition can be rewritten in terms of the slow-roll parameter $\eta$ as $M_{P l}^{2} m^{2} / \Lambda=\eta\left(\phi_{\min }\right)>3 / 4$. A rough estimate gives $\left|\dot{\phi}_{\min }\right| \sim \eta \phi_{0} \sqrt{3 \Lambda} / M_{P l}$. Therefore neglecting the kinetic term in the Friedmann equation (4.61) and using $H^{2} \simeq \Lambda / 3 M_{P l}^{2}$ is legitimate as long as $\eta^{2} \phi_{i}^{2} \ll M_{P l}^{2}$.

We want to apply this analysis to our potential (4.58) in the case this exhibits a minimum at $\phi=\phi_{\min }$. This could be the case for example if string theory does not allow for an arbitrary fine tuning of the effective parameters $C_{1}, C_{3 / 2}$ and $C_{2}$. Around $\phi_{\min }$, the potential can be approximated it with a harmonic oscillator $V \simeq V^{\prime \prime}\left(\phi-\phi_{\min }\right)^{2} / 2$ (see figure 4.4). We conclude that if $\eta\left(\phi_{\min }\right)<3 / 4$ the inflaton reaches the minimum $\phi_{\min }$ only asymptotically in infinite time. There is no graceful exit from inflation as there is no brane annihilation nor (damped) oscillations. The exponential expansion (with cosmological constant $\left.V\left(\phi_{\min }\right)\right)$ continues forever. On the contrary, if $\eta\left(\phi_{\min }\right)>3 / 4$, at the minimum $\dot{\phi}_{\min } \neq 0$ and there is the possibility to climb up the maximum, overshoot it and reach the tip of the throat where annihilation with the anti D3-brane will take place. 


\subsubsection{Uphill inflation}

In this section we study what happens when the inflaton rolls uphill. We will use our results to address the issue of overshooting a possible hill in the potential (see e.g. figure 4.1). Obviously the field will roll just for a short distance $\Delta \phi$ which depends on the initial speed $\dot{\phi}(0)$ that we take from the beginning to be $\dot{\phi}_{\text {min }}$. Let us study the simple potential

$$
V=\Lambda+c \phi,
$$

where $c$ is the positive slope. Under the simplifying assumption $\Lambda \gg c \phi$, the solution to the equation of motion is

$$
\phi(t)-\phi(0)=\Delta \phi=-\frac{c M_{P l}}{\sqrt{3 \Lambda}} t+\left(\frac{\dot{\phi}_{\min } M_{P l}}{\sqrt{3 \Lambda}}+\frac{c M_{P l}^{2}}{3 \Lambda}\right)\left(1-e^{-\sqrt{3 \Lambda} t / M_{P l}}\right) .
$$

The term linear in $t$ describes the constant speed rolling down that eventually dominates over the exponentially decreasing term. If the field starts with a positive $\dot{\phi}_{\min }$, it will climb up the hill for a distance

$$
\Delta \phi \simeq \frac{\dot{\phi}_{\min } M_{P l}}{\sqrt{3 \Lambda}}-\frac{c M_{P l}^{2}}{3 \Lambda}\left[1+\log \left(\frac{\sqrt{3 \Lambda} \dot{\phi}_{\min }}{c M_{P l}}\right)\right]
$$

in a time

$$
\Delta t \simeq \frac{M_{P l}}{\sqrt{3 \Lambda}} \log \left(1+\frac{\sqrt{3 \Lambda} \dot{\phi}_{\min }}{c M_{P l}}\right)
$$

before it stops and starts rolling down again. The number of e-foldings therefore is generically short unless the slope is exponentially small (if instead $\dot{\phi}_{\min }$ is very large, then $H$ is no more well approximated by a constant).

We would like to emphasize that an uphill motion is never slow roll. Even if $\epsilon \simeq 0=\eta$, when moving uphill $\ddot{\phi}$ is always very large (the motion is hampered both by the slope and by the Hubble friction) and can not be neglected. In fact the equation of motion is genuinely of second order and the uphill phase depends critically on the initial condition $\dot{\phi}_{0}$. On the other hand, the downhill slow-roll motion is an attractor and the solution eventually reaches it independently of $\dot{\phi}_{\min }$ (of course if the potential is of the slow-roll type).

We now have all the ingredients to address the question whether the inflaton will overshoot the maximum. We describe the part of the potential before the minimum $\phi>\phi_{\min }$ with the damped oscillator of section 4.6.3 (see figure 4.4). The key result is (4.63), the speed of the inflaton $\dot{\phi}_{\min }$ when it reaches $\phi_{\min }$. We then describe the uphill phase between maximum and minimum as a straight line. The estimate may seem very rough, so let 
us comment on it: if we take the steepness of our straight line ( $c$ in (4.64) ) to be the maximum steepness reached by the potential $V(\phi)$ between $\phi_{\max }$ and $\phi_{\min }$ then we have an upper bound. We show in the following that overshooting is possible in this extremel case; we conclude therefore that this is true also for the less steep non-straight uphill phase in the potential $V(\phi)$.

We use the result (4.63) as initial condition in (4.66). One can see that the time $t_{\text {min }}$ in (4.63) is always smaller than $\sqrt{3 \Lambda} / 2 M_{P l}^{2}$ so that, to get an order of magnitude estimate, we can neglect the exponential in that formula. Neglecting also numerical factors we take

$$
\dot{\phi}_{\min } \sim \phi_{0} \eta \sqrt{3 \Lambda} / M_{P l},
$$

where $\phi_{0}$ was the distance from the minimum of the initial position in the damped oscillatory phase of section 4.6.3. Substituting it into 4.66), we obtain

$$
\Delta \phi \sim \frac{\dot{\phi}_{\min } M_{P l}}{\sqrt{3 \Lambda}} \sim \phi_{0} \eta
$$

where we have used $c M_{P l}^{2} / 3 \Lambda \ll 1$ which is generic for our potential. We conclude that overshooting can happen, with $\eta \gtrsim 1$ and a comfortably natural choice $\phi_{0} \gtrsim \Delta \phi$ (also an initial $\dot{\phi} \neq 0$ at the beginning of the underdamped oscillatory phase will help to overshoot). Typically, one does not obtain a large number of e-foldings, see 4.67). We have to remember though that (4.67) is valid just when the uphill path is a straight line, in our case instead there is maximum, where the slope vanishes.

As an aside we comment on the intriguing correlation between a small cosmological constant and the underdamped oscillatory regime. A graceful exit from inflation typically requires that the inflaton reaches a minimum and starts oscillating and decaying (brane inflation is an interesting exception). In section 4.6.3 we have seen that the underdamped regime, leading to oscillation around the minimum, requires $\eta \gtrsim 1$. Equivalently, it requires that the cosmological constant $\Lambda$ is smaller than the inflaton mass $m$. Consider now an inflaton protected by some symmetry that therefore acquires an extremely small mass only from nonperturbative effects. Then an anthropic selection principle would apply: all universes with $\Lambda \gtrsim m^{2} M_{P l}^{2}$ would not have a graceful exit from inflation and would hence be empty.

Summarizing, if the inflaton potential is just $m^{2} \phi^{2}$, then anthropic arguments lead to an upper bound for the cosmological constant of order $\Lambda \lesssim m^{2} M_{P l}^{2}$. An extremely small inflaton mass might then explain the presence of a comparably small cosmological constant which may be responsible for today's measured cosmic accelerated expansion.

It would be interesting to study the features of a potential like (4.58). A preliminary observation is that, if an uphill phase is present, a largely non scale-invariant spectrum is produced. The spectral index during the uphill motion is given by

$$
n_{s}-1 \equiv \frac{d \log \delta_{\phi}}{d \log k} \simeq-\frac{\dot{\phi}}{H^{2}}-\frac{1}{H} \partial_{t}\left(\log \frac{\dot{\phi}}{H^{2}}\right)
$$


where the quantities on the right side have to be calculated at the time of horizon crossing. After some massage and using the Friedmann equations we obtain

$$
n_{s}-1 \simeq 4+\frac{c}{H \dot{\phi}}-\frac{\dot{\phi}}{H^{2}}+\frac{\dot{H}}{H^{2}} .
$$

The various terms do not cancel as it happens in the slow-roll regime; the reason can be traced back to the fact that $\ddot{\phi}$ is not small in this case. Per se, the absence of scaleinvariance is not a problem if the perturbations produced during the uphill phase are not those responsible for the CMB inhomogeneities, e.g. if the uphill phase takes place before or after 60 e-foldings prior to the end of inflation. These issues certainly deserve further study.

\subsection{Nice upliftings}

In the present chapter we have explicitly constructed a model where the interplay between moduli stabilization effects and threeshold corrections can produce a flat enough potential suitable for inflation. As we mentioned in section 4.1, there is another possibility to cure the $\eta$-problem of subsection 4.3 , i.e. use the inflaton dependence of the uplifting term to cancel the inflaton mass. We will not study in full details this alternative but we will explain the main idea and propose how it could be realized explicitly.

The setup of this section is therefore different from the one in the rest of the chapter. We will use a simplified notation which makes it easier to capture the features of the mechanism we are proposing. We consider a generalization of the KKLMMT model 65] reviewed in section 4.3 where the uplifting 4.19 has an a priori generic scaling $b$. We show that for a certain class of upliftings it is possible, allowing for fine tuning, to cancel the large mass of the inflaton that makes $\eta$ large and prevents slow-roll inflation.

Consider the potential

$$
V(\phi)=-\frac{\left|V_{F}\right|}{U^{2}}+\frac{\tilde{D}}{U^{b}}
$$

where ${ }^{8} U=1-\phi^{2} /\left(6 M_{P l}^{2}\right)$, the uplifting has a general $U$ dependence (and therefore inflaton dependence) parameterized by $b$ and $V_{K K L T}$ is similar to the KKLT [20] potential (2.36) but we have factorized out the inflaton dependence. Up to an overall factor

$$
V(\phi)=-\frac{1}{U^{2}}+\frac{D}{U^{b}}
$$

\footnotetext{
${ }^{8}$ Note that this definition is different from the one we used before in the rest of the chapter. The volume modulus $\tau$ has already been stabilized. Here the quantities $V_{K K L T}, D$ and $U$ differ from the ones in the rest of this chapter just by some factor that is unimportant for the discussion in this appendix. Also remember that the $\phi^{2}$ term in $U$ comes from the Kähler potential of the deformed conifold $k$ that, far away from the tip, is $k=r^{2}$ as in the singular case. See appendix B.4 for more details.
} 
Expanding for small $\phi$ we get

$$
V(\phi) \simeq-1+D+\left(-\frac{1}{3}+\frac{1}{6} D b\right) \frac{\phi^{2}}{M_{P l}^{2}}+\ldots .
$$

It is clear that the mass term for the inflaton can be made vanishing with an appropriate choice of the uplifting. The requirement is $D b=2$. The condition for a de Sitter vacuum is $D \gtrsim 1$ (up to terms of order $\phi^{2}$ ). An uplifting with $b<2$ allows to cancel at the same time the inflaton mass and uplift the AdS vacuum to a de Sitter one. In this minimal setup it is amusing to see that the cosmological constant is related to the mass of the inflaton. With respect to KKLMMT (see e.g. [84]), this reduces by one the number of string parameters that we need to fix by experiment. If, e.g. $b$ is very close to 2 but strictly smaller, then a small cosmological constant is equivalent to a small inflaton mass.

Unfortunately there is no well understood uplifting mechanism with $b<2$. An anti D3brane gives an uplifting that scales as $U^{-3}$. This generalizes for a Dp-brane wrapping a $p-3$ cycle to $U^{\frac{p-15}{4}}$ [88. If the cycle wrapped by the Dp-brane is at the tip of a warped throat, then the warp factor gives an additional $U$ factor. In KKLT for example, the anti D3-brane produces an uplifting term $U^{-2}$ as in 2.39). It would be intersting to study the configuration in which, e.g. a D5-brane wraps a nonvanishing two cycle at the tip of the throat ${ }^{9}$ leading to $b=3 / 2$.

\subsubsection{Nice downliftings}

In this section, we propose a variation on the theme of nice upliftings that we call nice downliftings. The advantage with respect to the nice upliftings is that with this mechanism we do not need any uplifting with $b<2$. The price to pay is that now we require at least two uplifting terms, and one of them has to be negative. Consider the following potential

$$
\begin{aligned}
V(\phi) & =-\frac{1}{U^{2}}+\frac{D_{1}}{U^{b_{1}}}-\frac{D_{2}}{U^{b_{2}}} \\
& =D_{1}-D_{2}-1+\left(-\frac{1}{3}+\frac{1}{6} D_{1} b_{1}-\frac{1}{6} D_{2} b_{2}\right) \phi^{2}+\ldots
\end{aligned}
$$

where $D_{1}, D_{2}>0$ and we neglect irrelevant factors. Then, to cancel the inflaton mass one has to require

$$
\begin{aligned}
& m_{\phi}^{2}=0 \quad \Rightarrow \quad D_{1}=\frac{2+D_{2} b_{2}}{b_{1}} \\
& V>0 \quad \Rightarrow \quad D_{1}>D_{2}+1 \text {. }
\end{aligned}
$$

\footnotetext{
$9^{\text {it }}$ is not necessary that it is a topologically non-trivial cycle but it could also be a metastable configuration, e.g. [89, 25].
} 
A simple example is $b_{1}=2, b_{2}=3$, then by fine tuning $D_{1}=1+D_{2} 3 / 2$, the inflaton mass becomes zero. It would be intersting to construct an explicit model that produces such "downliftings". Once we fine tune the parameters such that the quadratic term in $\phi$ becomes small, the quartic term has to be taken into account in the analysis. This term is not independent of the mass term and the overall potential is phenomenologically interesting.

We conclude this section with a remark: the nice uplifting scenario overcomes the $\eta$ problem of section 4.3 by allowing for fine tuning. This is not the most satisfactory solution because one wishes inflation was a generic prediction of the fundamental theory and not just an unprobable accident. Nevertheless, it would be interesting to study this kind of models to be able to explicitly quantify the required fine tuning. A naive, a priori (i.e. not based on any explicit setup) estimate of the required fine tuning might come out to be wrong in some explicit cases. An example is exactly what we have studied in the present chapter: a priori, it seemed quite reasonable to expect that it is possible to cancel the inflaton mass using the threshold corrections to the nonperturbative superpotential. But we have shown that [64, 66, 68, for two large classes of supersymmetric D7-brane embeddings, this is not possible for a wide range of inflaton values 10 .

\subsection{A summary on radial brane inflation}

Motivated by possible cosmological applications, we have studied the potential felt by a D3-brane in a warped conifold in the presence of a supersymmetrically embedded D7brane and an anti D3-brane sitting at the tip of the cone. The potential contains three terms: $V_{\mathrm{KKLT}}$, an uplifting term $V_{\mathrm{up}}$, and $\Delta V$, the latter arising when threshold corrections to the nonperturbative superpotential are taken into account. We have provided general formulae for the extremization of this potential in the angular and Kähler modulus directions. Once those moduli settle down at their minimum, we are left with an effective potential $V(\phi)$ for the canonically normalized radial D3-brane coordinate $\phi$.

We have studied the possibility to flatten $V(\phi)$ by fine-tuning, such that slow-roll Dbrane inflation can be embedded into a type IIB string-theory compactification with all the moduli fixed except the inflaton candidate. We have carried out a detailed analysis for two specific classes of supersymmetric D7-brane embeddings. In the throat (for small $\phi), \Delta V$ has a linear term in $\phi$ and otherwise depends on $\phi$ only via integer powers of $\phi^{3 / 2}$, whereas $V_{\text {KKLT }}$ and $V_{\text {up }}$ contain terms proportional to $\phi^{2}$. This means that the potential can be made flat only for a small range of $\phi$. Allowing for fine tuning, a flat inflection point can be generated. In this case the D3-brane dynamics sustains a prolonged stage of slow-roll inflation.

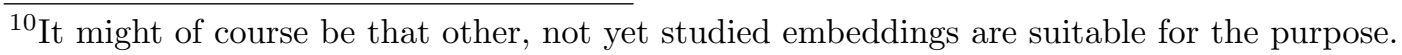


As we do not exactly know how much fine tuning in the effective parameters can be achieved by varying the discrete string theory parameters, we have also considered the issue of when, for a generic (non fine tuned) shape of the potential, the D3-brane can fall all the way down into the throat where it annihilates with the anti D3-brane.

The analysis of this chapter has taught us an important lesson that will motivate the construction of the model in the next chapter. The Coulomb attraction, which was supposed to drive brane inflation, is generically overwhelmed by the stronger forces generated from the F-term potential. Even in fine tuned cases, where the potential presents a flat region around an inflection point, one needs to take into account, at the same time, the Coulomb and the F-term potential. This means that the analyzes based on the simple brane-anti brane potential have to be reviewed if the brane system is embedded into a bona fide string compactification.

More importantly, we have learnt that a brane inflationary model driven by the Coulomb potential is even more difficult to construct than expected. This is because it is very hard to get rid of the strong moduli stabilization effects that generically dominate the dynamics of the inflating D3-brane. Therefore, it seems to be more natural that brane inflation, when it takes place, is driven by the omnipresent moduli stabilization force, which makes the presence of the anti brane superfluous. This is the idea we pursue in the next chapter where we construct a phenomenologically successful model of brane inflation without any anti D3-brane.

A comment on possible further corrections is in order. Quantum corrections, from loop or $\alpha^{\prime}$ effects, are generically subleading in the KKLT stabilization scenario, the reason being a very small $W_{0}$ (see section 6.2). But the force exerted by the effective potential $V(\phi)$ on the inflaton is hierarchically weaker than the one responsible for the stabilization of the closed string moduli (that is why we can talk about an effective $V(r)$ in the first place). Therefore, we expect that that quantum corrections will have sizable effects on warped brane inflation scenarios analyzed here. This issue could be addressed along the lines of [90, 91, 92]. We will not do this in the present work. On the other hand, in chapter 6 we pursue a very similar goal, i.e. investigate the effects of quantum corrections with respect to the tree level analysis, for a particle physics model instead of for an inflationary one. 


\section{Angular brane inflation}

The material presented in this chapter is mainly based on [57]. We introduce and study a new model of brane inflation based on the motion of a (spacetime-filling) D3-brane on the tip of a warped deformed conifold looking for inflationary trajectories. Figure 5.1 gives a cartoon of the model.

The chapter is organized as follows. In section 5.1 we give some motivations to investigate such a model and confront it with the model of the last chapter. After describing in section 5.2 the general features of inflation at the tip, in section 5.3 we set the stage and calculate the scalar potential for a generic D7 embedding. In section 5.4 we specify the embedding and find an explicit potential; we discuss the stabilization of the other moduli (Kähler modulus and radial displacement) and introduce the uplifting. The final result of this first part is the potential appearing in (5.19). This is the starting point for the inflationary analysis of section 5.5: we consider both the slow-roll and the DBI regimes. In section 5.6 we generalize our analysis of DBI inflation, we present a no go result and describe how to evade it. Finally, we give a summary of angular brane inflation. We collect several technical details in the appendix $\mathrm{C}$.

\subsection{Motivations}

In this section we want to critically discuss several features of the brane inflation model considered in the last chapter to see what can be improved and how. Eventually we will propose (and study in the rest of the present chapter) a new model of brane inflation [57] that we will call, for reasons that will become obvious, inflation at the tip. Our starting point is again the idea that inflation could be driven by a scalar field representing the position of a spacetime-filling D3-brane [49] in the compact manifold; our considerations will be restricted to the framework of type IIB flux compactifications reviewed in chapter 2 .

In the radial model of the last chapter, one of the key elements is an anti D3-brane which sits at the tip of a warped throat. It plays a threefold role: it produces a Coulomb term in the inflaton potential, it annihilates with the inflating D3-brane providing a reheating mechanism ${ }^{1}$ and, finally, it breaks supersymmetry and can provide an effective

\footnotetext{
${ }^{1}$ Actually the tachyon condensation driving the annihilation process might as well be responsible for
} 
4-dimensional de Sitter (or Minkowski) space. Let us separately analyze these issues and emphasize the available alternatives.

1. Without an anti D3-brane, a different mechanism from brane annihilation has to be responsible for reheating. Although we do not address this issue here, we would like to make a few remarks. One of the original motivations for brane inflation [49] was to obtain an efficient reheating mechanism; the interesting feature of brane brane collision (contrary to annihilation) is that after the collision the kinetic energy of the relative motion is almost entirely transferred into modes living on the newly created stack of branes and the creation of bulk (Kaluza Klein) modes is negligible. The process of brane collision has been carefully studied in [93, 94] and this expectation has been confirmed. On the other hand, a thorough phenomenological analysis of this reheating mechanism has not yet been performed. It would be very interesting to have a quantitative estimate of the efficiency of this reheating process. In this chapter we propose a model, inflation at the tip, where a possibility for the end of inflation is precisely the collision of several D3-branes (in the common minimum of the potential); the above reheating mechanism (to which we will refer as "brane trapping") would hence be naturally embedded.

2. In addition to the original anti D3-brane at the tip of a warped throat [20], several other mechanisms have been proposed to break supersymmetry and uplift the effective 4-dimensional AdS vacuum to a dS one. We will therefore try to keep our analysis as general as possible, without specifying the uplifting mechanism. We will find at the end that some constraints on the scaling of the uplifting are indeed present.

In the model described in the last chapter, (and in most models based on [65]), the D3brane motion takes place along the radial direction of a warped conifold. We have seen in the discussion of the $\eta$-problem (in section 4.3) that the radial position is typically a direction too steep for slow-roll inflation. The reason is that the radial position turns out to be a conformally coupled scalar in the 4-dimensional effective action. This motivates the study of the angular motion ${ }^{2}$. In [75] for example, the possibility of slow-roll inflation for a pair of a D3-brane and an anti D3-brane at the tip, i.e. separated only in the angular directions, was considered. The authors found a negative result that a posteriori can be understood as follows. The brane-anti brane separation is bounded from above by the size of the tip that is extremely small. The Coulomb potential becomes very steep for small brane separation which makes slow-roll inflation impossible. When the potential is

graceful exit from inflation. This is very model dependent. E.g. in the setup analyzed in [85, 86, it was found that the CMB data prefer the end of inflation due to the failure of the slow-roll conditions rather than due to tachyon condensation.

${ }^{2}$ Works who studied inflation driven by an exclusively angular motion of branes are [89, 25, 75], who found a negative result. In [95, 96, 97, 98, and [99, 100, 101, 102, the cosmological implications of a combined angular and radial motion were considered. 
induced exclusively by moduli stabilization it depends crucially on the compactification (and it is not "universal" as the Coulomb potential) and can therefore be made flat with an appropriate choice of the parameters (if and when this requires fine tuning will be discussed further in the chapter).

A final point on which we want to comment is the DBI action. For the phenomenological study of the model in the last section, we approximated the DBI kinetic term with a canonically normalized one. This is a good approximation as long as the speed of the inflaton and/or the warping are not too large. If inflation takes place close to the tip of a warped deformed conifold the warping is much larger than in the middle of the throat, which is the region interesting for the radial brane inflation model of chapter 4 . Hence, for inflation at the tip, the DBI kinetic term can more easily become relevant and the interesting effects of DBI inflation can be obtained.

The idea of DBI inflation [50, 51] is that, although the inflaton potential is not flat (in the slow-roll sense), inflation can nevertheless last long enough because the DBI kinetic term is very different from the canonical one (when the warping and/or the speed of the inflaton are large) and it imposes an upper bound on the inflaton speed. The DBI kinetic term is determined by the warp factor that, for the warped conifold, is a function of the radial position. If the motion takes place along the angular directions, at constant radius, as in inflation at the tip, the warp factor is constant. In a certain sense, this gives the simplest DBI kinetic term possible, which makes the inflationary analysis particularly easy. In fact, in section 5.5.3, we will study analytically a DBI model with a potential like in Natural Inflation [103, 104]. This will lead to the interesting observation that the phenomenological constraint that the axion decay constant has to take superplanckian values is relaxed in presence of a DBI kinetic term.

\subsection{Inflation at the tip}

In the rest of the chapter we will see how all the issues we have mentioned in the last section can be addressed in a new interesting way. In particular we propose a study a specific model that we will call inflation at the tip.

This model (schematically depicted in figure 5.1) consists of a spacetime-filling D3-brane moving along the tip of a warped deformed conifold which is an $S^{3}$. The potential depends on three scalar fields (the three angles of $S^{3}$ ) and the overall volume. It is generated by the F-term which is determined by the supersymmetric embedding of a stack of spacetimefilling D7-branes. The choice of a particularly symmetric D7-brane embedding gives rise to a very simple scalar potential. In different regions of the parameter space this potential induces different cosmological evolutions. For example, we will show in subsection 5.4.1 that a quite general choice of parameters leads to the Natural Inflation potential; in some fine tuned regions one can also obtain some kind of hilltop potential. Given the simplicity 


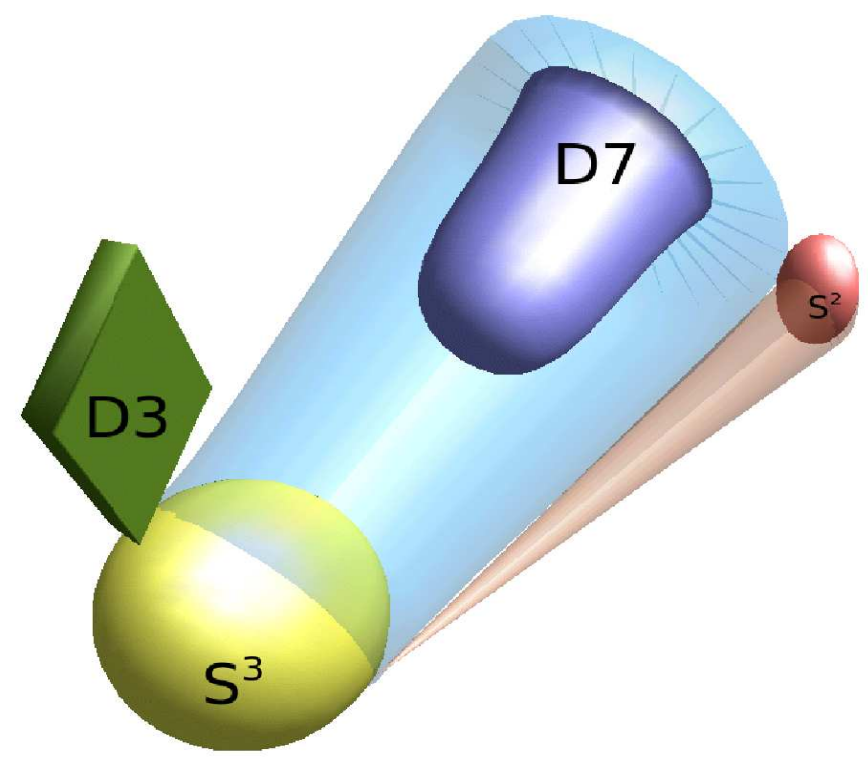

Figure 5.1: A cartoon of inflation at the tip, where we zoom in close to the tip of the warped throat: the simplest model has just one D3-brane and a very symmetric D7-brane embedding.

of the potentials we find, we do not need to rely on numerical methods for the inflationary analysis and also for the DBI inflation important results can be obtained analytically.

First we look for slow-roll inflation, i.e. we neglect the DBI corrections to the canonical kinetic term. We find that, fine tuning stringy parameters, the potential can be made flat enough. Phenomenologically, this corresponds to a model of the hilltop type [105, 106], with $n_{s} \simeq 0.94$, negligible tensor modes and an adjustable scale of inflation $\Lambda^{1 / 4} \sim d \times 10^{-3}$ where $d$ is given in $(5.29)$ and is generically subplanckian. In light of our discussion in chapter 3, we conclude that inflation at the tip is a successful inflationary model.

We look as well at the DBI regime but we can not find a totally successful model in the simplest setup. In fact, we show in section 5.5.3 that DBI inflation can produce the right perturbations (with interesting non-Gaussianity signature) but not enough e-foldings. Then we argue that, considering more generic embeddings (as depicted in figure 5.7), one can obtain a viable inflationary model in which DBI and slow-roll phases alternate. For example the perturbations can be produced in the DBI regime, while the rest of the 60 observable e-foldings take place in the slow-roll regime. We leave a detailed study of these DBI-slow-roll alternating models for future work. 


\subsection{F-term potential for a D3-brane at the tip}

In this and the next section we start our study of the inflation at the tip introduced in the last section. We calculate the potential for a single D3-brane moving along the tip of a warped deformed conifold [107] (reviewed in appendix A) in the framework of type IIB flux compactifications (introduced in chapter 2). In this section we set the stage and obtain the general result $(5.10)$ for the potential. In section 5.4 we consider a simple embedding and obtain the more explicit result (5.19).

Our starting point is similar to the one of chapter 4. We consider type IIB string theory compactified on a Calabi-Yau threefold; following [20] (reviewed in section 2.6), we assume that the complex structure moduli and the dilaton have been stabilized by fluxes [8] at a scale hierarchically higher than the scale of inflation. We again make the simplifying assumption that there is only one Kähler modulus $T$. The $N=1$ supergravity scalar potential was given in (4.10) and the Kähler potential $K$ [80] in 4.11.

We assume that a warped throat [107] is present such that, deep inside it, $k$ is well approximated by the Kähler potential of the deformed conifold. In the last chapter we considered the region of the throat far away from the tip, where the deformed conifold asymptotes the singular conifold. On the other hand, we will now focus on the tip and hence we can not ignore the deformation anymore (see appendix A).

The conifold is defined by ${ }^{3}$ the following hypersurface in $\mathbb{C}^{4}$

$$
\sum_{A=1}^{4}\left(z^{A}\right)^{2}=\varepsilon^{2}
$$

and its tip by

$$
\sum_{A=1}^{4}\left|z^{A}\right|^{2}=\varepsilon^{2}
$$

Putting these equations together and writing them in terms of $z_{A}=x_{A}+i y_{A}$, with $A=1, \ldots, 4$, one finds that the tip is an $S^{3}$ embedded in a real slicing of the $z_{A}$ complex coordinates

$$
\text { tip : } \quad \sum_{A=1}^{4} x_{A}^{2}=\varepsilon^{2}, \quad y_{A}=0 .
$$

Close to the tip, the Kähler potential of the deformed conifold takes the form [108, 75]

$$
k(z, \bar{z})=k_{0}+c \varepsilon^{-2 / 3}\left(\sum_{A=1}^{4}\left|z_{A}\right|^{2}-\varepsilon^{2}\right),
$$

\footnotetext{
${ }^{3}$ Note that here we use another set of coordinates for the conifold as compared to the one used in chapter 4. The relation is given in A.3 of appendix A
} 
where $c=\frac{2^{1 / 6}}{3^{1 / 3}} \simeq 0.77$. Hence, at the tip $k(z, \bar{z})$ is just a constant; this implies that $U=T+\bar{T}-\gamma k_{0}$ does not depend on the position of the D3-brane on the tip. This is a crucial difference with respect to radial brane inflation models (as the one of chapter 4 ) because in that case $k \propto r^{2} \propto \phi^{2}$ (or $k \propto r^{3} \propto \phi^{3}$ for the region close to the tip) and this is the origin of the $\eta$-problem of section 4.3 . In other words, contrary to the radial position, the angular position does not correspond to a conformally coupled scalar in 4-dimensions.

Only three of the four $z_{A}$ are independent and should express the fourth of them as a function of the other coordinates. After doing this, one can verify [75] that at the tip $k$ is stationary in all directions, i.e. $\partial_{z^{A}} k=0$. Thanks to this property, the Kähler metric and its inverse [66, evaluated at the tip, take a simple block diagonal form:

$$
\begin{aligned}
G_{I \bar{J}} & =\frac{3}{\kappa^{2} U^{2}}\left(\begin{array}{cc}
1 & -\gamma k_{\bar{\jmath}} \\
-\gamma k_{i} & U \gamma k_{i \bar{\jmath}}+\gamma^{2} k_{i} k_{\bar{\jmath}}
\end{array}\right)=\frac{3}{U^{2} \kappa^{2}}\left(\begin{array}{cc}
1 & 0 \\
0 & U \gamma k_{i \bar{\jmath}}
\end{array}\right) \\
G^{\bar{I} J} & =\frac{\kappa^{2} U}{3}\left(\begin{array}{cc}
U+\gamma k_{\bar{l}} k^{\bar{l} h} k_{h} & k_{\bar{l}} k^{\bar{l} j} \\
k^{\bar{l}} k_{l} & \gamma^{-1} k^{\bar{\imath} j}
\end{array}\right)=\frac{\kappa^{2} U}{3}\left(\begin{array}{cc}
U & 0 \\
0 & \gamma^{-1} k^{\bar{\imath} j}
\end{array}\right),
\end{aligned}
$$

where $i$ and $j$ run on three of the four $z_{A}$. Choosing $z_{1}, z_{2}, z_{3}$ as independent coordinates and $z_{4}^{2}=\varepsilon^{2}-\sum_{i=1}^{3} z_{i}^{2}$, from (5.4) we obtain

$$
\begin{array}{lll}
k_{i \bar{\jmath}}=\frac{c}{\varepsilon^{2 / 3}}\left(\delta_{i \bar{\jmath}}+\frac{z_{i} \bar{z}_{j}}{\left|z_{4}\right|^{2}}\right) & \longrightarrow \quad k_{i \bar{\jmath}}=\frac{c}{\varepsilon^{2 / 3}}\left(\delta_{i \bar{\jmath}}+\frac{x_{i} x_{j}}{\varepsilon^{2}-\sum x_{h}^{2}}\right) \\
k^{\bar{i} j}=\frac{\varepsilon^{2 / 3}}{c}\left(\delta^{\bar{\imath} j}-\frac{z_{i} \bar{z}_{j}}{\sum\left|z_{A}\right|^{2}}\right) & \text { at the tip } & \longrightarrow \quad k^{\bar{i} j}=\frac{\varepsilon^{2 / 3}}{c}\left(\delta^{\bar{i} j}-\frac{x_{i} x_{j}}{\varepsilon^{2}}\right),
\end{array}
$$

where on the right side we used that $z_{A}=\bar{z}_{A}=x_{A}$, at the tip.

The superpotential, in light of the discussion of section 4.2, is given in 4.3):

$$
W=W_{0}+A_{0} g(z)^{1 / n} e^{-a T},
$$

where, as before, $W_{0}$ and $A_{0}$ are (after integrating out the dilaton and the complex structure moduli) treated as constants and $g(z)$ is the embedding function defining the cycle $\Sigma$ where the physics responsible for the non-perturbative corrections lives.

The F-term potential (4.10) for $K$ given in (4.11), using (5.5) and (5.6), takes the form

$$
V_{F}=\frac{\kappa_{4}^{2}}{3 U^{2}}\left[U\left|W_{, T}\right|^{2}-3\left(\bar{W} W_{, T}+\text { c.c. }\right)+\frac{1}{\gamma} k^{\bar{i} j} \bar{W}_{, i} W_{, j}\right] .
$$

For the superpotential in (4.3), this becomes

$$
\begin{array}{r}
V_{F}=\frac{\kappa_{4}^{2}}{3 U^{2}}\left[\left(U a^{2}+6 a\right)|A|^{2} e^{-a(T+\bar{T})}\right. \\
+3 a\left(\overline{W_{0}} A e^{-a T}+\text { c.c. }\right) \\
\left.+\frac{1}{\gamma} k^{\bar{\imath} j} \bar{A}_{, \bar{\imath}} A_{, j} e^{-a(T+\bar{T})}\right] .
\end{array}
$$


The minimization of the axion works as in subsection 4.4.1 (see e.g. [64]), leading to the final result

$$
\begin{aligned}
V_{F}= & V_{K K L T}+\Delta V \\
= & \frac{\kappa_{4}^{2} 2 a|A| e^{-a \tau}}{U^{2}}\left(\frac{1}{6} a U|A| e^{-a \tau}+|A| e^{-a \tau}-\left|W_{0}\right|\right) \\
& +\frac{\kappa_{4}^{2} e^{-2 a \tau}}{3 U^{2} \gamma} k^{\bar{i} j} \bar{A}_{\overline{,}} A_{, j} .
\end{aligned}
$$

We would like to stress that this form of the potential is valid only for the D3-brane at the tip, where the Kähler metric (5.6) is block diagonal. In this case the potential depends on the variables $\tau, x_{1}, x_{2}, x_{3}$. For a generic position, e.g. in the middle of the throat, the expression would be much more complicated. In [75], the supersymmetric vacua of this potential were studied solving $D_{I} W=0$ for two classes of embeddings. Depending on the choice of the embedding, the set of supersymmetric vacua can be either empty, a point, one- or two-dimensional.

The symmetries of the problem give us an important insight. With $W_{\mathrm{np}}$ set to zero, the F-term potential for a D3-brane is constant because of the no-scale structure. Nonperturbative corrections break the no-scale structure and induce a non-trivial potential for the D3-brane. On the other hand, as long as we ignore threshold corrections, i.e. we consider $A$ as a constant in 4.3 , the angular directions at the tip (where $k=k_{0}$ ) are still flat directions. In other words, the the F-term potential enjoys an $S O(4)$ symmetry acting on the coordinates $z_{A}=x_{A}$, i.e. the isometry group of $S^{3}$. Threshold corrections to the non-perturbative break this symmetry via the embedding function $g(z)$. An appropriate choice of $g(z)$ can lead to a very symmetric and simple potential $V$. In the next section we provide an explicit example of this where $g(z)$ breaks $S O(4)$ to $S O(3)$ and the potential $V$ depends on the D3-brane position $z_{A}=x_{A}$ only via a single real field $\phi$. In section 5.6 we comment on the implications of a generic $g(z)$.

\subsection{Kuperstein embeddings}

We now calculate the potential in (5.10) for a class of particularly symmetric embeddings. We also review the result of the radial stabilization (performed in appendix C.2 that guaranties that the D3-brane at the tip is at a local minimum in the radial direction. Our final result is (5.19) (valid for large volume and when $\varepsilon / \mu \gg 1$, i.e. when the D7-branes are not too close to the tip), which will be the starting point for the inflationary analysis of section 5.5 .

As we already said, the tip of the deformed conifold is an $S^{3}$ which can be described as the real slicing of the $z_{A}$ coordinates plus the constraint (5.3). The $S O(4)$ symmetry of $S^{3}$, acting naturally on $x_{1}, x_{2}, x_{3}, x_{4}$, is broken once a certain embedding function $g$ (defining the divisor $\Sigma$ where the stack of D7-branes is wrapped) is chosen. 


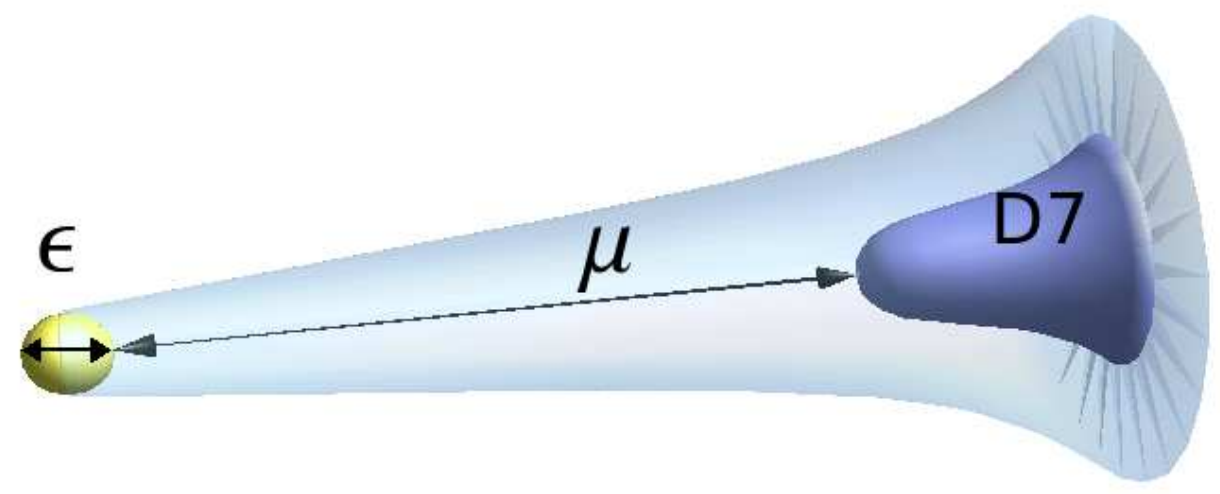

Figure 5.2: The figure describes the geometrical meaning of the parameters $\mu$, i.e. the distance of the stack of D7-branes to the tip, and $\varepsilon$, i.e. the size of the tip.

We have already introduced, in section 4.5, two families of supersymmetric D7 embeddings. Here we concentrate on the Kuperstein embeddings [73] defined in 44.5). For a generic $\tilde{g}(x)$ these embeddings preserve an $S O(2)$ subgroup of the $S O(4)$ symmetry, corresponding to rotations in the $z_{2}-z_{3}$ plane.

For this family of embeddings, the first term in the potential, $V_{K K L T}$ is easily calculated substituting $A=A_{0} g(x)^{1 / n}$ in (5.10). For the term $\Delta V$, a straightforward calculation using (5.7) leads to

$$
\Delta V=\frac{\kappa_{4}^{2}|A(x)|^{2} e^{-2 a \tau} \varepsilon^{2 / 3}}{3 c n^{2} U^{2} \gamma\left|\tilde{g}-x_{1}\right|^{2}} \cdot\left[1-\frac{x_{1}^{2}}{\varepsilon^{2}}+4 x_{23} \tilde{g}^{\prime 2}\left(1-\frac{x_{23}}{\varepsilon^{2}}\right)+4 \tilde{g}^{\prime} \frac{x_{23} x_{1}}{\varepsilon^{2}}\right],
$$

where the prime indicates derivative with respect to $z_{23}$.

\subsubsection{A simple case}

For certain choices of $\tilde{g}$, the potential can exhibit very flat regions (e.g. flat inflection points); the difficulty is that multi-field inflaton analysis is in general required. In section 5.6 we will further comment on a generic $\tilde{g}$, but a thorough analysis is left for future investigation. On the contrary in the following, we will study a particular symmetric embedding that leads to single-field inflation. As we did in section 4.5.2, here we focus again on the simplest case when $\tilde{g}\left(z_{23}\right)=\mu$ in 4.5 and, without lost of generality, we take $\mu$ to be real and positive. Again, by rescaling $A_{0}$ (by a factor $\mu^{1 / n}$ ), we can take the Kuperstein embedding as in (4.51). An advantage of this choice is explained by the following consideration. As depicted in figure 5.2 , the parameter $\mu$ denotes the deepest radius reached by the stack of D7-branes $r_{D 7}=\mu^{2 / 3}$. We are interested in the case where the D7-branes do not reach close to the tip, such that $\varepsilon / \mu \ll 1$ is a good expansion 
parameter. With the rescaled embedding 4.51, we have then $A(z)=A_{0}+\mathcal{O}(\varepsilon / \mu)$, i.e. the threshold corrections give rise to terms in $W_{\mathrm{np}}$ suppressed by $\varepsilon / \mu$.

Written out explicitly, the non-perturbative superpotential is

$$
W_{\mathrm{np}}=A(z) e^{-a T}=A_{0}\left(1-\frac{z_{1}}{\mu}\right)^{1 / n} e^{-a T} .
$$

As $g(z)=g\left(z_{1}\right)$, the $S O(4)$ symmetry of the potential is broken to $S O(3)$ that acts naturally on $z_{2}, z_{3}, z_{4}$. Using (5.12), the potential (5.10) becomes

$$
\begin{aligned}
V_{K K L T} & =\frac{\kappa_{4}^{2} 2 a e^{-a \tau}}{U^{2}}\left[\frac{1}{6} a U\left|A\left(x_{1}\right)\right|^{2} e^{-a \tau}+\left|A\left(x_{1}\right)\right|^{2} e^{-a \tau}-\left|W_{0} A\left(x_{1}\right)\right|\right] \\
\Delta V & =\frac{\kappa_{4}^{2} e^{-2 a \tau}}{3 U^{2} \gamma} k^{\overline{1} 1} \bar{A}\left(x_{1}\right)_{, \overline{1}} A\left(x_{1}\right)_{, 1} \\
& =\frac{\kappa_{4}^{2}\left|A\left(x_{1}\right)\right|^{2} e^{-2 a \tau}}{3 c n^{2} \mu^{2} U^{2} \gamma} \varepsilon^{2 / 3}\left(1-\frac{x_{1}^{2}}{\varepsilon^{2}}\right)\left(1-\frac{x_{1}}{\mu}\right)^{-2},
\end{aligned}
$$

and depends only on two real variables, $\tau$ and $x_{1}$. Thanks to the $S O(3)$ symmetry, two of the three independent coordinates $x_{i}$ with $i=1,2,3$ are flat directions of the scalar potential, i.e. $x_{2}$ and $x_{3}$. Some effects, such as for example corrections from the bulk 4 , will eventually stabilize these directions. In the inflationary analysis of section 5.5 we will therefore assume that the D3-brane starts and stays at a minimum in the $x_{2}$ and $x_{3}$ directions and moves only along $x_{1}$. This technical assumption is almost ubiquitous in the brane inflation literature. We used it as well in chapter 4 to neglect the angular directions and focus on the radial motion. The effects of these "flat" directions can be investigated in a second step, once a successful model of inflation is found.

In principle, the kinetic term $K_{i \bar{\jmath}} \partial z_{i} \partial \bar{z}_{j}$ mixes all the $z_{i}$ 's. For our case, a more convenient parameterization of the $S^{3}$ is

$$
\begin{array}{ll}
z_{1}=x_{1}=\varepsilon \cos \phi, & z_{2}=x_{2}=\varepsilon \cos \phi \sin \psi \sin \theta, \\
z_{3}=x_{3}=\varepsilon \sin \phi \sin \psi \sin \theta \quad, z_{4}=x_{4}=\varepsilon \cos \theta \sin \psi,
\end{array}
$$

where $\phi$ runs from 0 to $\pi$. This choice leads to the diagonal metric

$$
d s^{2}=c \varepsilon^{4 / 3}\left[d \phi^{2}+\sin ^{2} \phi\left(d \theta^{2}+\sin ^{2} \theta d \psi^{2}\right)\right] .
$$

In these new coordinates, the potential in (5.13) and (5.14) is just a function of $\tau$ and $\phi$ and the kinetic term is diagonal.

\footnotetext{
${ }^{4}$ Notice that, as argued in [75, bulk effects are in general subleading with respect to the potential we are considering here. They are important only when the potential has a completely flat direction, as it is the case for the $x_{2}$ and $x_{3}$ directions.
} 
We write now the potential in these new coordinates. We expand $V_{K K L T}$ and $\Delta V$ for $\varepsilon / \mu \ll 1$ :

$$
\begin{aligned}
V_{K K L T} \simeq & \frac{2 \kappa_{4}^{2}\left|A_{0}\right| a e^{-a \tau}}{U^{2}}\left(\frac{1}{6}\left|A_{0}\right| a U e^{-a \tau}+\left|A_{0}\right| e^{-a \tau}-\left|W_{0}\right|\right) \\
& +\frac{2 \kappa_{4}^{2} \varepsilon\left|A_{0}\right| a e^{-a \tau}}{U^{2} n \mu}\left(\frac{1}{3}\left|A_{0}\right| a U e^{-a \tau}+2\left|A_{0}\right| e^{-a \tau}-\left|W_{0}\right|\right) \cos \phi+\ldots \\
\Delta V \simeq & \frac{\kappa_{4}^{2}\left|A_{0}\right|^{2} e^{-2 a \tau} \varepsilon^{2 / 3}}{3 c n^{2} \mu^{2} U^{2} \gamma} \sin ^{2} \phi+\ldots
\end{aligned}
$$

We know from section 2.6, that the first term in (5.16), gives rise to an AdS minimum [20] (that can be trusted for small $W_{0}$ ) and that the other terms are suppressed with respect to the first by a factor $\varepsilon /(n \mu)$ and $\varepsilon^{2 / 3} /(n \mu)^{2}$, respectively. Hence if we want to have inflation we need an uplifting. In chapter 4 , we chose for concreteness an anti D3-brane at the tip of the warped throat. As we mentioned, this is not the only possibility.

There are several alternatives in the literature, that can be divided into two groups: Dterm [81, 109, 110, 111, 112, 113, 114, 115, 116] or F-term upliftings [82, 117, 118, 119, 120, 121, 122, 123, 124, 125, 126, 83. To keep our analysis general, following our discussion in section 5.1, we will assume in the sequel that a term

$$
V_{u p}=\frac{D}{U^{b}}
$$

is present, without specifying its origin. For the moment $D$ and $b$ are arbitrary positive numbers (the former has the dimension of an energy density, the latter is dimensionless). This is analogous to what we did when we discussed nice upliftings in section 4.7 .

For inflation at the tip, we will see in section 5.4 .2 and in appendix C.2 that the concrete value of $b$ is very important to ensure the radial stability of the D-brane, i.e. that the D-brane at the tip is at a local minimum in the radial direction. In addition, $D$ has to be such that the cosmological constant at the end of inflation is very small (see section 2.6). Notice that $V_{u p}$ does not depend on the D3-brane position at the tip, contrary to the radial brane inflation case where the radial dependence of $V_{u p}$ can even be used to flatten the potential as we saw in section 4.7 .

Putting all the ingredients together, the scalar potential takes the form

$$
\begin{aligned}
V(\tau, \phi) & =V_{u p}+V_{K K L T}+\Delta V \\
& \simeq \Lambda(\tau)+B(\tau) \cos \phi+C(\tau) \sin ^{2} \phi+\ldots,
\end{aligned}
$$

where $\Lambda, B$ and $C$ are positive, with the dimension of a (mass) ${ }^{4}$ and can be obtained comparing (5.18) with (5.16); the subleading terms are suppressed at least by a factor $\varepsilon / \mu$. The potential (5.18) depends on two real variables $\tau$ and $\phi$, but the former gets a 
much larger mass than the latter and we can integrate it out. As we show in appendix C.1. the minimum $\tau_{c r}(\phi)$ in the $\tau$ direction has a very mild dependence on $\phi$. In fact, calculating $V\left(\tau_{c r}(\phi), \phi\right)$ this dependence produces terms of the same order as those that we have neglected in (5.18). We are therefore allowed to substitute $\tau \simeq \tau_{c r}$ in $\Lambda, B$ and $C$ and study the single-field potential

$$
V(\phi) \simeq \Lambda+B \cos \phi+C \sin ^{2} \phi+\ldots
$$

Notice that this is very different from the radial brane inflation case. There we showed that the inflaton dependence of $\tau_{c r}$ has to be taken into account correctly; in fact, it changes even qualitatively the shape of the inflaton potential, as can be seen in figure 4.2). The reason for this difference is that the Kähler potential depends on the radial position (see e.g. (B.23) and (B.24) ) but not on the angular position at the tip (see (5.4)).

It is important now to determine the relative size of the three coefficients above. To do this we need to know the value of $\tau_{c r}$. Analogously to chapter 4 (see also appendix C.1), a useful reparameterization of $D$ and $W_{0}$ is

$$
\begin{aligned}
W_{0} & =-A_{0} e^{-a \tau_{0}}\left[1+\frac{1}{3} a\left(2 \tau_{0}-k_{0}\right)\right], \\
D & =\beta \frac{1}{3}\left|A_{0}\right|^{2} a^{2} e^{-2 a \tau_{0}}\left(2 \tau_{0}-k_{0}\right)^{b-1} .
\end{aligned}
$$

Then, at leading order, the minimum of the volume is (compare with 4.30 and $(\mathrm{B} .12$ in the radial brane inflation case)

$$
\tau_{c r}(\phi) \simeq \tau_{0}+\frac{b}{2} \frac{\beta}{a^{2} \tau_{0}}+\frac{\varepsilon}{a \mu n} \cos \phi+\frac{\varepsilon^{2 / 3}}{2 c a^{3} n^{2} \mu^{2} \tau_{0} \gamma} \sin ^{2} \phi+\ldots
$$

Substituting $W_{0}, D$ and $\tau_{c r}$ into $\Lambda, B$ and $C$ obtained from (5.16), and neglecting terms subleading in the $\varepsilon / \mu$ and large volume expansion, we are left with

$$
\begin{aligned}
& \Lambda \simeq \frac{\kappa_{4}^{2}\left|A_{0}\right|^{2} a^{2} e^{-2 a \tau_{0}}}{6 \tau_{0}}\left[(\beta-1)-\frac{b^{2} \beta^{2}}{4\left(a \tau_{0}\right)^{2}}+\ldots\right] \\
& B \simeq \frac{\kappa_{4}^{2}\left|A_{0}\right|^{2} a e^{-2 a \tau_{0}} \varepsilon}{6 n \mu \tau_{0}^{2}}\left[(b \beta-3)+\frac{b \beta(14-3 b \beta)}{4 a \tau_{0}}+\ldots\right] \\
& C \simeq \frac{\kappa_{4}^{2}\left|A_{0}\right|^{2} e^{-2 a \tau_{0}} \varepsilon^{2 / 3}}{12 c n^{2} \mu^{2} \gamma \tau_{0}^{2}}+\ldots
\end{aligned}
$$

We will see in the following that we want to take $\beta$ so that the cosmological constant after inflation is negligible. This implies $(\beta-1)=\mathcal{O}\left(\tau_{0}^{-2}\right)$. Then, from the above equations, it is clear that some subtleties arise in the case $b=3$ because then the factor $(b \beta-3)$ introduces an additional suppression that is not taken into account by the $\varepsilon / \mu$ and large volume expansion. On the other hand, $b=3$ is quite interesting because explicit uplifting mechanisms exist with this scaling [81, 109, 110, 111, 112, 113, 114, 115, 116, 82, 117, 
118, 119, 120, 121, 122, 123, 124, 125, 126, 83. A part from the anti D3-brane at the tip of a warped throat that scales with $b=2$, we are not aware of any other explicit model with $b \neq 3$; such scaling could be interpreted ${ }^{5}$ as if the physics responsible for the susy-breaking were localized on a $\mathrm{D} p$-brane with $p \neq 3$. In this case the uplifting would naively scale as $U^{(p-15) / 4}$. Other considerations about the role of the uplifting are given in the next section, in section 4.7 and in appendix C.2.

In the following we will separately discuss the case $b=3$ and $b \neq 3$. Let us compare $B$ and $C$ to know which is the leading $\phi$-dependent term. The result follows straightforwardly from $(5.24)$ and $(5.25)$ remembering that $(\beta-1)=\mathcal{O}\left(\tau_{0}^{-2}\right)$ :

$$
C \ll B \Longleftrightarrow \begin{cases}(\text { anc } \gamma \mu) \varepsilon^{1 / 3} \gg \frac{4}{15} a \tau_{0} & \text { for } b=3 \\ (\text { anc } \gamma \mu) \varepsilon^{1 / 3} \gg 1 & \text { for } b \neq 3,\end{cases}
$$

i.e. in the above regimes $C$ is negligible and $V$ in (5.19) takes the form of the Natural Inflation potential [103, 104]. We will see in the next section that in this regime slow-roll inflation is impossible ${ }^{6}$. In section 5.5.3 we will study the DBI regime for the Natural Inflation potential. On the other hand, there is an interesting case in which $C$ is nonnegligible, i.e. the fine tuned case where $C=B / 2$. As we will see in section 5.5.1, with this choice the scalar potential supports slow-roll inflation. The fine tuning is achieved when the inequality in (5.26) is fulfilled (in the case $b=3$ or $b \neq 3$ respectively), modulo a factor one half.

In what follows we estimate $(a n c \gamma \mu)^{3}$. This will tell us how generic the Natural Inflation regime $B \gg C$ (defined in (5.26) ) is. In addition we will learn whether there is any obstruction from the string theory point of view to achieve the fine tuning $B=2 C$.

From the definition of $a$, it follows an $=2 \pi$. The quantity $\mu^{2 / 3}$ indicates the smallest radial value $r_{\mu}$ reached by the stack of D7-branes (see figure 5.2). To be able to trust the threshold corrections to the non-perturbative superpotential (4.2), $r_{\mu}$ has to be well inside the warped conifold. In the spirit of [127], the conifold geometry ceases to be a good description of the compact manifold roughly when the warping $h$ in the 10dimensional metric (4.6) becomes of order one. When the KS solution [107] is embedded in a compact Calabi-Yau as in GKP [8], we get for the warp factor $h(r) \simeq(R / r)$ where $R^{4} \equiv(27 \pi / 4) g_{s} N \alpha^{\prime 2}$. Hence an upper bound on $\mu$ is

$$
\mu<R^{3 / 2}=\left(\frac{27 \pi}{4} g_{s} N\right)^{3 / 8} .
$$

The parameter $\gamma$ (defined in (4.11)) can be obtained by comparing the sugra with the DBI action. The result is $\gamma=\tau_{0} T_{3} /\left(3 M_{P l}^{2}\right)$ [68]. Expressing it in string units we get

\footnotetext{
${ }^{5}$ We thank L. McAllister for suggesting this interpretation [88. For some further comments, see section 4.7 .

6 As it will become clear in section 5.5.1 Natural Inflation can not be achieved because the axion decay constant (that appears when we consider a canonically normalized kinetic term, see (5.29) is always small in Planck units
} 
$\gamma=(2 \pi)^{4} g_{s} /\left(6 \sqrt{\tau_{0}}\right)$, where we have used ${ }^{7} V_{6} \sim \tau_{0}^{3 / 2}$. Putting all the ingredients together we obtain

$$
(a \gamma \mu n)^{3} \sim 10^{11} \frac{g_{s}^{3}\left(g_{s} N\right)^{9 / 8}}{\tau_{0}^{3 / 2}}\left(\alpha^{\prime}\right)^{-3 / 4} .
$$

For the choice of parameters $\left\{g_{s}=0.1, N=10^{4}, \tau_{0}=100\right\}$, we get $(\text { an } \gamma c \mu)^{3} \simeq 3 \times 10^{8}$. In view of the conditions in (5.26), e.g. for $b=3$, a value $\varepsilon \gg 6 \times 10^{-8}$ allows to neglect $C$ in the potential. This value of $\varepsilon$ corresponds to a warp factor at the tip $h_{0} \sim 2 \times 10^{19}$. For such a moderately warped throat, we are therefore left with the Natural Inflation potential [103, 104]. For fine tuned values of $\varepsilon$ saturating this bound, the potential is plotted in figure 5.4 and will be analyzed in section 5.5.2.

\subsubsection{Radial stability}

Until now we have calculated the dependence of the potential on the position of the D3brane at the tip. Before using this result to produce a stage of inflation, we have to check that the radial direction $r$ is not tachyonic, i.e. that if the D3-brane starts at the tip, it will stay there. We leave the details of the calculation to the appendix C.2, here we state and comment on the results.

We consider separately the case $C \ll B$, that, as we will see, can lead to DBI inflation, and the case $B=2 C$ that, allowing for fine tuning, leads to a phenomenologically successful slow-roll inflation. The radial stability is determined by the sign of $\partial_{r} V$ evaluated at the tip $r=\varepsilon^{2 / 3}$.

From the fact that the $k$ in $(5.4)$ depends on $r$ and from the discussion of section 4.7 , it is easy to envisage that the uplifting will play an important role in the radial minimization. This is confirmed by the explicit calculation. In both cases $C \ll B$ and $2 C \simeq B$, the tip is a local minimum in the radial direction if the volume scaling $b$ of the uplifting is larger than or equal to 3 . On the other hand, for $b<3$ the radial stability depends on the angular position. In this case, in some regions of the tip the radial derivative is positive, in some others negative. To avoid this complication, in the following we just consider the case in which an uplifting with $b \geq 3$ is performed.

\subsection{Inflationary analysis}

For the inflationary analysis, it is easier to work with a canonically normalized inflaton field:

$$
\phi_{\text {can }}=\varepsilon^{2 / 3} \sqrt{T_{3}} c \phi \equiv d \phi
$$

\footnotetext{
${ }^{7}$ We make this choice for the sake of simplicity; it is straightforward to generalize this estimate to the case in which the volume of the Calabi-Yau manifold is larger than the volume of the conifold.
} 
Now the potential can be written in the form

$$
V(\phi)=\Lambda+B \cos \frac{\phi}{d}+C \sin ^{2} \frac{\phi}{d}
$$

where, to keep the notation simple, here and in the following we use again $\phi$ to indicate $\phi_{\text {can }} ; \Lambda, B$ and $C$ were defined in (5.23), (5.24) and (5.25). For the inflationary analysis, we can think of $\Lambda, B$ and $C$ as some constants with the dimension of an energy density that are determined in terms of stringy parameters.

If we want to be left with a vanishing or very small cosmological constant after inflation, i.e. when $\phi \simeq \pi d$, then we have to choose the uplifting in (5.30) such that $\Lambda-B=0$. Using (5.23) and (5.24), we can solve this equation for $\beta$. The solution can be expanded in $\varepsilon / \mu$ and for large volume; the leading term is determined by $\Lambda=0+\mathcal{O}(\varepsilon / \mu)$ because $B$ is suppressed by a factor $\varepsilon / \mu$ with respect to $\Lambda$. Hence, a zero consmological constant requires $\beta=1+\mathcal{O}\left(\varepsilon / \mu, 1 /\left(a \sigma_{0}\right)^{2}\right)$, i.e. $\beta$ very close to one . Using $\Lambda=B$, the potential becomes

$$
V(\phi)=\Lambda\left(1+\cos \frac{\phi}{d}\right)+C \sin ^{2} \frac{\phi}{d},
$$

where $0 \leq \phi / d \leq \pi$. There are two interesting regimes to analyze: $\Lambda \gg C$ and $2 C \simeq \Lambda$. In the first case the potential reduces to the Natural Inflation potential [103, 104]

$$
V(\phi)=\Lambda\left(1+\cos \frac{\phi}{d}\right)
$$

Originally this potential was derived for an axionic field, in which case the parameter $d$ is the axion decay constant; we sometimes borrow this terminology.

Notice that both in the regime $\Lambda=B \gg C$ and $\Lambda=B \simeq 2 C$ our expansion in (5.16) is still valid. The terms we neglected in the expansion of $V_{K K L T}$ and $\Delta V$ are suppressed by a factor $\varepsilon / \mu$. These subleading terms can become important close to the minimum of the potential $\phi \simeq \pi d$, where our leading order potential (5.31) approaches zero. Anyways, when the D3-brane reaches that region, inflation is already over, therefore we do not expect these corrections to have any influence on our analysis.

The potential (5.31) is the starting point for the phenomenological analysis of this section. The kinetic term for $\phi$ (see (5.45) ) comes from the DBI action (2.18). We will therefore divide our analysis into two parts: first, we investigate in section 5.5.1 and 5.5.2 the slow-roll regime in which the DBI kinetic term reduces to the canonical one, as we did in chapter 4. Second, in section 5.5.3 we investigate the relativistic regime in which the DBI action is responsible for a behavior very different from the slow-roll case.

The search for slow roll is in turn divided into two parts: first in section 5.5.1 we consider (5.31) in the regime $\Lambda \gg C$ which reduces to the Natural Inflation potential (5.32). A thorough analysis of this potential with a canonical kinetic term has already been performed in [103, 104]; we review the constraints on the only two parameters $\Lambda$ and 
$d$ imposed by the comparison with WMAP3 data (see figure (5.3)). Expressing $\Lambda$ and $d$ in terms of stringy parameters we show that our model of Natural Inflation can not fulfil the constraints. Basically, this is due to the impossibility of obtaining a large axion decay constant in the string theory model. This fact is true also if one tries to use the assisted inflation mechanism [63] considering the collective motion of many D3-branes at the tip. These difficulties are similar to those found in the case of axionic N-flation [128, 129, 130, 62, 46, 131].

The second part of the slow-roll analysis is in section 5.5.2, where we show that the potential (5.31) in the fine tuned regime $\Lambda \simeq 2 C$ becomes very flat close to the top (see figure 5.4). With this fine tuning, we can have a phenomenologically successful slow-roll inflation with $n_{s} \simeq 0.94$, negligible tensor modes and the scale of inflation $(\Lambda)^{1 / 4} \sim$ $d \times 10^{-3}$.

In section (5.5.3) we come to the DBI analysis. In this case the regime $2 C \simeq \Lambda$ does not possess additional interesting features. Therefore we limit our analysis to the potential 5.32). As can be seen from the action in (5.45), the DBI kinetic term introduces a new parameter: $f$ (in string theory it is given by the warp factor times the D3-brane tension). An interesting result is that with a DBI kinetic term, contrary to what happens with a canonical one, the CMB data do not require a superplanckian value for $d$ anymore. This feature might be relevant for the task of embedding a phenomenologically successful Natural Inflation into string theory.

The embedding of DBI Natural Inflation in string theory that we proposed in the last section can not satisfy the phenomenological bounds on $\Lambda, a$ and $f$. In section 5.6, we prove that this is actually true for any potential at the tip provided that it satisfies the DBI conditions (5.51) and (5.52) during the whole duration of inflation. The reason is that, if the motion is exclusively relativistic (DBI regime), then inflation at the tip can not last more than a few e-foldings, which is not enough to solve the initial condition problems of section 3.2. This no-go result rules out a large class of potentials and gives us an important criterion to look for a successful model: the potential needs to have, at least somewhere, flat regions where instead of the DBI conditions, the slow-roll conditions are fulfilled. For inflation at the tip, this kind of alternating potentials might arise, e.g. considering a general Kuperstein embedding, i.e. a general $\tilde{g}$ in 4.5 .

\subsubsection{Slow-roll Natural Inflation}

In this section we consider the inflaton action 8

$$
S=-\int d^{4} x \sqrt{-g}\left[\frac{1}{2} \partial^{\mu} \phi \partial_{\mu} \phi+\Lambda\left(1+\cos \frac{\phi}{d}\right)\right],
$$

\footnotetext{
${ }^{8}$ Notice that we are using the $(-,+,+,+)$ convention for the metric.
} 


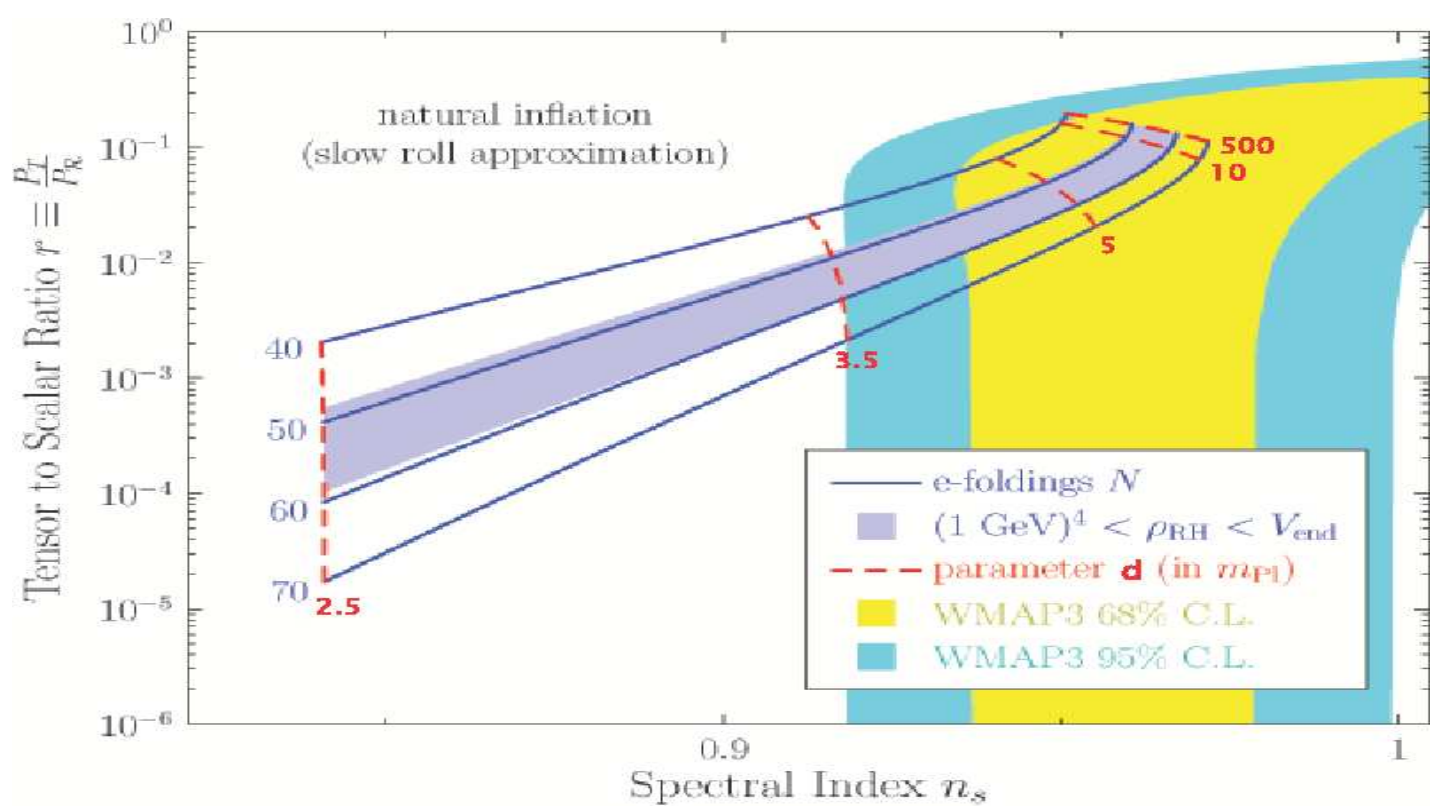

Figure 5.3: WMAP3 data and the predictions of natural inflation are shown in the $n_{s}-r$ plane. The figure is taken from [103].

which is the one felt by a D3-brane moving at the tip of a warped deformed conifold when a stack of D7-branes wraps a 4-cycle defined by the supersymmetric Kuperstein embedding (4.5) with constant $\tilde{g}=\mu$ and the volume is fixed à la KKLT. This form is valid at leading order in the large volume and $\varepsilon / \mu$ expansion (i.e. when the D7-branes are not too close to the tip). Also we are in the (large) region of the parameter space where we can neglect the $C$ term in (5.31). The effects of this term will be considered in the next section.

The potential (5.33) was already studied in [103, 104]. The model successfully reproduces WMAP3 data [29] if $9 \sim m_{G U T}$ and $d>0.7 \sqrt{8 \pi} M_{p l} \simeq 3.5 M_{p l}$, as summarized in figure 5.3. In this section we show that in the string model it is not possible to have a superplanckian $d$. Hence, as regards slow-roll inflation, our model, in the regime $\Lambda \gg C$, is not phenomenologically successful.

For $d \gg M_{P l}$ the natural inflation approximates the $\phi^{2}$ chaotic inflation; in fact the CMB perturbations are produced close to $\phi \simeq \pi d$ where the two potentials are indistinguishable. For $d<M_{P l}$ on the other hand, inflation has to start very close to the top of the potential to get enough e-foldings. The problem is that at the top one has $|\eta| \sim\left(M_{P l} / d\right)^{2} \gtrsim 1$, i.e. the slow-roll conditions are not satisfied. In the next section we will see how this problem can be cured in a different regime $(2 C \simeq \Lambda)$ from the one considered in this section $(C \ll \Lambda)$ and allowing for fine tuning.

\footnotetext{
${ }^{9} \mathrm{As}$ it is common in the string cosmology literature, we indicate with $M_{P l}$ the reduced Planck mass: $8 \pi G_{N}=M_{P l}^{-2}$. To compare it with the Planck mass one has to multiply the latter with $\sqrt{8 \pi} \simeq 5.01$.
} 
We now show that in the regime of validity of our stringy model, the constraint $d \gtrsim 3.5 M_{P l}$ can not be satisfied. $d=\varepsilon^{2 / 3} \sqrt{T_{3} c}$ appears because we work with a canonically normalized inflaton field and it was introduced in (5.29). The assisted inflation idea [63] was used for the first time in string brane inflation in [132] (for assisted axionic string inflation see [128, 129, 130, 62, 46, 131]); it can also be naturally embedded in the present model of inflation at the tip. In fact, if $N_{D 3}$ D3-branes are present at the tip, $d$ gets multiplied by a factor $\sqrt{N_{D 3}}$, which goes in the right direction to reproduce the CMB data. The details of how to obtain this result are left to the appendix C.3.

We now look for a set of stringy parameters that lead to $\sqrt{N_{D 3}} d>3.5 M_{p l}$. Several constraints are imposed by consistency. One comes from considering the backreaction of the $N_{D 3}$ D3-branes on the deformed conifold geometry. For the warped deformed conifold to be a solution of Einstein's equations, the following 3-form fluxes have to be present

$$
\frac{1}{(2 \pi)^{2} \alpha^{\prime}} \int_{A} F_{3}=M, \quad \frac{1}{(2 \pi)^{2} \alpha^{\prime}} \int_{B} H_{3}=-K
$$

This solution possesses $K$ times $M$ D3-brane charge. As long as the number $N_{D 3}$ of D3-branes responsible for inflation is much smaller than the background D3 charge $K M$, we can neglect their backreaction on the geometry at leading order.

The requirement $d \sqrt{N_{D 3}}>3.5 M_{p l}$ leads to

$$
N_{D 3}>15.8 \varepsilon^{-4 / 3} \frac{M_{p l}^{2}}{T_{3}} .
$$

The D3-brane tension is as usual given by

$$
T_{3}=\frac{1}{(2 \pi)^{3} g_{s} \alpha^{\prime 2}} .
$$

Here we have to consider the effect of the warping, i.e. that the string scale at the tip of the throat is $M_{s} a_{0}$ with $a_{0}=\varepsilon^{2 / 3} /\left(g_{s} M \alpha^{\prime}\right)^{1 / 2}$ the warp factor at the tip. Therefore we have

$$
\text { at the tip: } \quad \frac{T_{3}}{M_{p l}^{4}}=\frac{(2 \pi)^{11} g_{s}^{3}}{4 V_{6}^{2}} a_{0}^{4}=\frac{(2 \pi)^{11} g_{s}}{4 V_{6}^{2} M^{2}} \frac{\varepsilon^{8 / 3}}{\alpha^{\prime 2}} .
$$

The volume (in string units) can be written as the sum of the warped conifold volume ${ }^{10}$ plus the rest of the Calabi-Yau manifold, thus an obvious lower bound is [89, 25]

$$
V_{6}>V_{\text {conifold }} \simeq\left(\frac{2 \pi}{3}\right)^{3}\left(\frac{27 \pi}{4} g_{s} M K\right)^{(3 / 2)} .
$$

\footnotetext{
${ }^{10}$ The conifold is non-compact, therefore its volume is infinite. What we mean here is the volume of the warped conifold once this is cut at a radius $r \sim R$, where the warp factor becomes of order one and the conifold does not approximate the geometry of the Calabi-Yau anymore, in the spirit of [127]
} 
Substituting this and 5.37$)$ into 5.35 one obtains

$$
N_{D 3}>K^{3 / 2}\left(g_{s} M\right)^{5 / 2} \frac{\alpha^{\prime 3}}{\varepsilon^{4}} 18 .
$$

For the supergravity approximation to be valid, the radius of the tip has to be large in string units, therefore $\left(g_{s} M\right)^{1 / 2} \gg 1$. Even in the most favorable case of very shallow throats, a superplanckian value of $d$ requires $N_{D 3} \gg K M$ which makes it inconsistent to neglect the backreaction.

Even if one could take into account the backreaction of the inflating D3-branes, we expect that the situation would not improve much. To see this, suppose that $N_{D 3} \gg M K$; then the AdS radius scales as $R^{4} \sim N_{D 3}$ and the volume of the conifold as $V_{6} \sim N_{D 3}^{3 / 2}$, which gives a parametric dependence similar to the one in (5.38). Again it seems to be impossible to fulfil (5.35), i.e. to get $d$ of order one or larger. This difficulty is very similar to the one found in axionic natural assisted inflation [128, 129, 130, 62, 46, 131], where no controllable string compactification has been found with $d>M_{P l}$.

\subsubsection{Slow-roll hilltop inflation}

In this section we consider the potential (5.31) for a single D3-brane at the tip, in the regime in which $C$ is non-negligible. We find that it is possible to fine tune the string parameters such that the potential at the top becomes very flat. This hilltop model [105, 106] gives rise to a prolonged stage of inflation and is perfectly compatible with WMAP3.

Let us expand the potential close to the top where $\phi \ll d$. The result is

$$
V \simeq 2 \Lambda+\frac{1}{2 d^{2}}(2 C-\Lambda) \phi^{2}+\frac{1}{24 d^{4}}(\Lambda-8 C) \phi^{4}+\mathcal{O}\left(\frac{\phi^{6}}{d^{6}}\right)
$$

For a generic $C \sim \Lambda$ the slow roll parameter $\eta$ (defined in (3.5) together with $\epsilon$ ) is nowhere small as we mentioned in the last section. But if we fine tune $C=\Lambda / 2$, close enough to the top of the potential we have $\epsilon \ll \eta \ll 1$. In terms of stringy parameters, this fine tuning can be achieved, e.g. in the case $b=3$, varying the fluxes such that $\varepsilon=(2 \pi \mu \gamma c)^{-3} 4 a \tau_{0} / 15$ (that we estimated in (5.26)). Notice that this can be achieved independently of the fine tuning of the cosmological constant, i.e. $\Lambda=B$, that is obtained varying the uplifting, i.e. $\beta$.

As the cancellation $2 C-\Lambda$ becomes more and more precise, the potential at the top becomes flatter and flatter as can be seen from figure 5.4. For simplicity, in the following we study the case in which the cancellation is precise enough to neglect the mass term ${ }^{11}$,

\footnotetext{
${ }^{11}$ The case in which $C \sim \Lambda / 2$ and both the quadratic and quartic terms are of the same order could also be considered, see for example [133].
} 

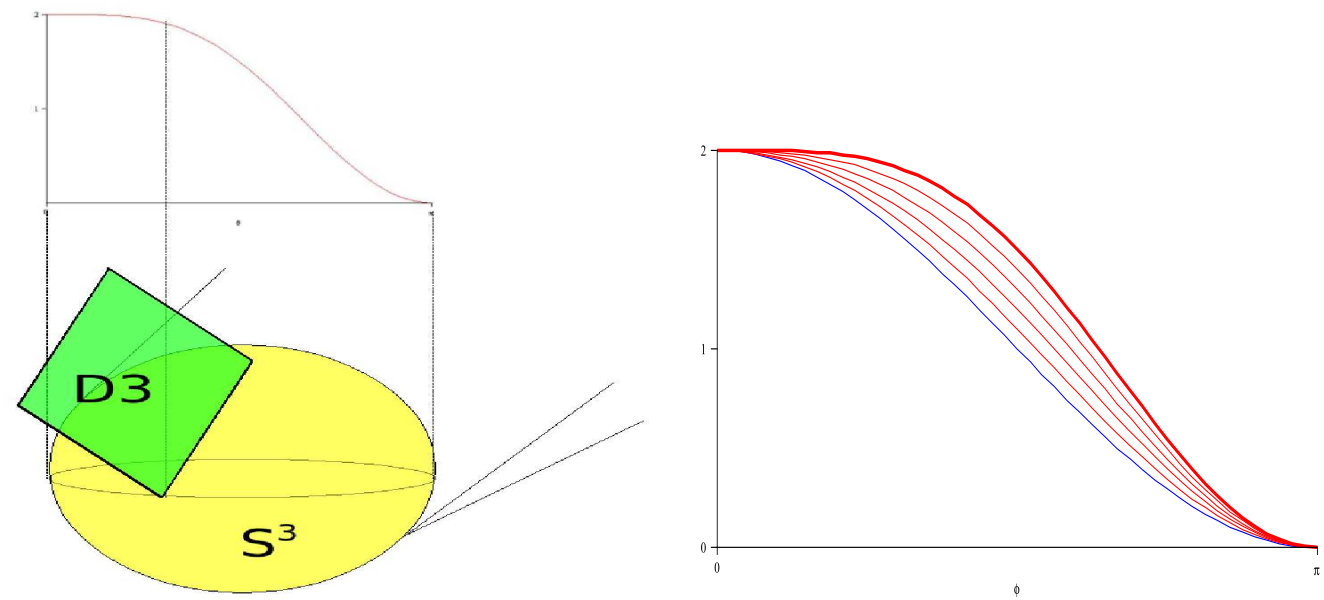

Figure 5.4: On the left: a cartoon of the hilltop inflation model. On the right: we show explicitly how the fine tuning works. The potential (5.31) is plotted for $C / \Lambda=\{0.5,0.4,0.3,0.2,0.1,0\}$. The thick line corresponds to $C=\Lambda / 2$, i.e. a perfect cancellation of the mass term in 5.40 . The lowest (blue) line corresponds to $C=0$, i.e. the natural inflation potential (5.32).

such that the potential is well approximated by

$$
V \simeq 2 \Lambda\left(1-\frac{1}{16 d^{4}} \phi^{4}\right)
$$

The COBE normalization (3.10) imposes the constraint $\Lambda^{1 / 4} \sim d \times 10^{-3}$ [33]. As we discussed in the last section, typically in our string theory model $d<M_{P l}$; this implies that the scale of inflation can at most be somewhat smaller than the GUT scale. The number of e-foldings $N_{e}$ before the end of inflation when the scales of the CMB perturbations left the Hubble horizon is given by (3.11] [33]. In our case, the most relevant term is the one including the scale of inflation, which, for hilltop models, can be quite low. We therefore have

$$
N_{e} \simeq 60-\ln \frac{10^{16} \mathrm{Gev}}{V^{1 / 4}} \simeq 58.4+\ln \frac{d}{M_{P l}}
$$

For example, $d \sim 2 \times 10^{-4} M_{P l}$ gives $N_{e} \simeq 50$. For the potential ${ }^{12}$ (5.41) $\eta \simeq 2 /\left(3 N_{e}\right)$ and, as it is typical for small field models, $\epsilon \ll \eta$. Therefore we have a prediction for the scalar spectral index $n_{s} \simeq 1+2 \eta \sim 0.94$ in good agreement with WMAP3 [29].

To summarize, our model of inflation at the tip gives the potential in (5.41) provided that ${ }^{13}$ we can fine tune the warp factor at the tip with enough precision to make the mass term negligible. Then the potential (5.41) gives predictions in good agreement with the

\footnotetext{
${ }^{12}$ Generically for a potential $V \sim 1-\mu \phi^{p}$ with $p \geq 3$ we have $\eta=-(p-1) /\left[(p-2) N_{e}\right][33$ ]

${ }^{13}$ The problem of fine tuning the uplifting to get the right cosmological constant is somehow a different problem from the one of embedding inflation in string theory and we do not address it here.
} 
experiment if $\Lambda \sim d^{4} \times 10^{-12}$. In a numerical investigation we found explicit values that can satisfy this requirement. We conclude that, allowing for fine tuning, inflation at the tip can provide a phenomenologically-viable prolonged stage of slow-roll inflation.

\subsubsection{DBI Natural Inflation}

In the last two sections (and in chapter 4), we looked for slow-roll inflation and we were therefore allowed to approximate the kinetic term of the D3-brane with a canonical one. In this section, we investigate the possibility of obtaining successful DBI inflation, i.e. with a potential that is too steep to fulfil the slow-roll conditions.

For the angular position of spacetime-filling D3-brane, the kinetic term in the DBI action 2.18 becomes

$$
S=-\int d^{4} x \sqrt{-g}\left(f^{-1} \sqrt{1+f \partial^{\mu} \phi \partial_{\mu} \phi}-f^{-1}\right)
$$

where $f=h / T_{3}$ is the warped D3-brane tension. When $f \partial^{\mu} \phi \partial_{\mu} \phi \ll 1$ this reduces to the canonical kinetic term (plus a constant term), as we have assumed in the last two sections. When, on the contrary, $f \partial^{\mu} \phi \partial_{\mu} \phi \sim 1$ all the higher terms in the Taylor expansion of the square root become relevant. Now the kinetic term behaves very differently from the canonical case; e.g. there is an upper limit $\dot{\phi}^{2}<f^{-1}$, where, having inflation in mind, we assume homogeneity. In analogy with the usual notation of special relativity, a useful parameter to quantify how close we are to the maximal allowed speed i: ${ }^{14}$

$$
\gamma=\frac{1}{\sqrt{1-f \dot{\phi}^{2}}}
$$

which diverges for $\dot{\phi}^{2}<f^{-1}$.

In sections 5.3 and 5.4 we have seen that the D3-brane feels a non-trivial potential along the tip. Therefore the effective action we want to study is

$$
S=-\int d^{4} x \sqrt{-g}\left(f^{-1} \sqrt{1+f \partial^{\mu} \phi \partial_{\mu} \phi}+V(\phi)-f^{-1}\right)
$$

where $V(\phi)$ is the potential in (5.31).

A comment about $f$ is in order. As we stressed in 5.1, the warp factor $f$ (or $h$ equivalently) is constant everywhere at the tip because it depends only on the radial position. The actual value is determined by the complex structure moduli and the dilaton which in turn are stabilized by fluxes. Hence, for the inflationary analysis we will treat $f$ as a constant

\footnotetext{
${ }^{14}$ Clearly $\gamma$ here has nothing to do with the one in section (5.3), e.g. in 4.11. As we separate the inflationary from the string theory analysis, we hope no confusion will arise.
} 
with the dimension of a (mass $)^{-4}$. Notice that this is a new and interesting feature of inflation at the tip. The other string theory DBI models considered until now were concerned with the radial motion in which case $f \propto r^{-4}$ is strongly inflaton dependent. Choosing the angular position as inflaton candidate has the advantage of considerably simplifying the DBI analysis.

In this section we concentrate on the regime in which the $C$ term in 5.31 is very small and can be neglected leaving the Natural Inflation potential in (5.32). As argued in section 5.4.1. this is quite a natural situation. Anyways, as we will see in the next section, as long as we are interested just in DBI inflation, the precise shape of the potential is not important.

The effective action (5.45) has three parameters: $d, f$ and $\Lambda$. We focus on the regime $d \ll M_{P l}$, which is what happens generically in string theory. We study how these three parameters are constrained by WMAP3 data. The result is that the phenomenological constraint for DBI inflation can not be fulfilled in the string theory model. In section 5.6 we generalize this negative result to any potential provided that it is nowhere slow-roll flat. As we describe in 5.6.1, what would work is a potential that alternates slow-roll flat regions with steep ones. To obtain such a potential in our model of inflation at the tip, we should consider more generic embeddings than the simple one with constant $\tilde{g}$ that leads to the potential $(5.31)$.

En passant we obtain an interesting result: as we reviewed in section 5.5.1, the consistency with CMB data requires that the axion decay constant $d$ of Natural inflation with a canonical kinetic term takes superplanckian values. We will see that in the presence of a DBI kinetic term, this constraint is relaxed and a viable (DBI) inflationary model occurs for $d$ as small as $0.04 M_{P}{ }^{15}$.

\section{$\mathrm{DBI}$ in a nutshell}

The idea of DBI inflation [50, 51] is that, for a D-brane moving in a warped space, the maximal allowed speed can be considerably smaller than the speed of light. This allows to obtain inflation even when the inflaton potential is not as flat as required by the standard slow-roll conditions. In the following we collect the relevant steps for a DBI inflation analysis, and refer the reader to the original papers [50, 51] for further discussions.

Given the action (5.45), the energy density $\rho$ and pressure $p$ in the perfect fluid approxi-

\footnotetext{
${ }^{15}$ This statement refers to the effective action 5.45 per se, without the need to specify its high energy origin. On the other hand, if we try to obtain (5.45) from string theory, we can not arbitrarily vary the parameters and in fact, as we said, a successful DBI Natural Inflation can not be realized in our string theory model.
} 
mation are

$$
\begin{aligned}
& \rho=\frac{\gamma}{f}+\left(V-f^{-1}\right), \\
& p=-\frac{1}{f \gamma}-V+f^{-1} .
\end{aligned}
$$

Using the Hamilton-Jacobi formalism, where $\phi$ plays the role of the time variable, the equivalents of the Friedmann equation and of the equation of motion for $\phi$ are

$$
\begin{aligned}
\dot{\phi} & =-\frac{2 H^{\prime}}{\sqrt{M_{P l}^{-4}+4 f H^{\prime 2}}}, \\
H^{2} & =\frac{\rho}{3 M_{P l}^{2}} .
\end{aligned}
$$

Defining

$$
\epsilon_{D B I}=\frac{2 M_{P l}^{2}}{\gamma}\left(\frac{H^{\prime}}{H}\right)^{2}
$$

we have that inflation lasts as long as $\epsilon_{D B I}<1$. In analogy with the slow-roll case, we have two DBI conditions [50, 51]

$$
\begin{aligned}
\frac{V^{3 / 2}}{\left|V^{\prime}\right| M_{P l}} \sqrt{3 f} \gg 1 & \Rightarrow & H^{2} \simeq \frac{V}{3 M_{P l}^{2}}, \\
\frac{V^{\prime 2}}{3 V} f M_{P l}^{2} \gg 1 & \Rightarrow & \gamma \gg 1 .
\end{aligned}
$$

They guarantee that the energy density is dominated by the potential term and that the motion is relativistic respectively.

\section{DBI Natural Inflation}

In this section we study the DBI regime of a brane moving along the tip under the Natural Inflation potential (5.32). We are in the (large) region of parameter space where the term $C$ in (5.31) is very small and can be neglected. We will find that this model of exclusively DBI inflation at the tip can not give enough e-foldings. As we will see in section 5.6, this is true for any potential provided that it does not possess any slow-roll region.

For the Natural Inflation potential (5.32), as long as the conditions (5.51) and (5.52) are fullfilled, we have

$$
H=\frac{\sqrt{\Lambda\left(1+\cos \frac{\phi}{d}\right)}}{\sqrt{3} M_{P l}} .
$$



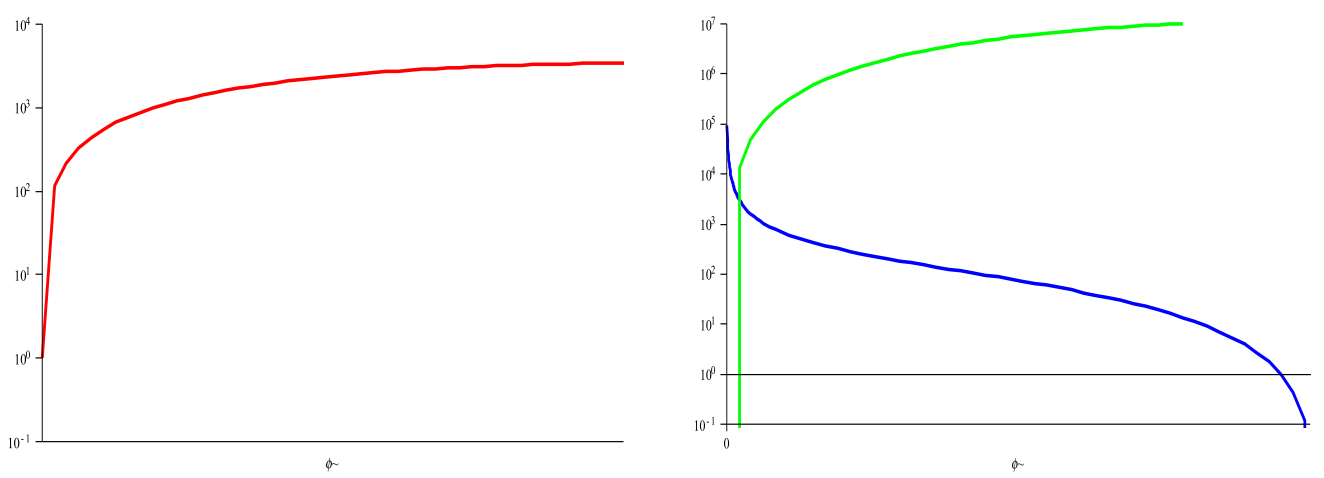

Figure 5.5: On the left: $\gamma$ is plotted for the Natural Inflation potential; it grows monotonically from 1 to $(2 f \Lambda / 3)^{1 / 2} M_{P l} / d$. On the right: the plot shows that the DBI condition (5.51) (darker line) and (5.52) (lighter line) for the Natural Inflation potential (5.32) with $d \ll M_{P l}$ are satisfied everywhere except for the two extremal regions, $\phi \simeq 0$ and $\phi \simeq d \pi$.

Using (5.48) we can easily obtain $\dot{\phi}$. The number of e-foldings (defined in (3.4)) is then given by

$$
N_{e}=\int_{t_{i}}^{t_{f}} H d t=\int_{\phi_{i}}^{\phi_{f}} \frac{H}{\dot{\phi}} d \phi .
$$

where the suffixes $i$ and $f$ refer to the beginning and the end of inflation.

Close to the top of the potential the inflaton moves non-relativistically (as found also in [134]). This regime is uninteresting for us because, as we said, in that region $\eta \sim$ $-\left(M_{P l} / d\right)^{2}$ which for small $d / M_{P l}$ gives a very red spectrum. Therefore we look ${ }^{16}$ further away from the top where the motion becomes relativistid ${ }^{17}$. As we mentioned, the importance of relativistic effects can be estimated evaluating $\gamma$. Substituting the solution (5.48) for $\dot{\phi}$ in the definition (5.44), we get

$$
\gamma=\frac{1}{\sqrt{1-f \dot{\phi}^{2}}}=\frac{M_{P l}}{d \sqrt{3}} \sqrt{f \Lambda\left(1-\cos \frac{\phi}{d}\right)+3 d^{2}},
$$

that is plotted in the left part of figure (5.5). $\gamma$ grows monotonically from one (nonrelativistic motion) to $M_{P l} \sqrt{f \Lambda} / d$.

To avoid unobserved large non-Gaussianities in the CMB, the perturbations have to be produced when $\gamma \lesssim 22$ [50, 51]. This happens close to the top (but far enough to be in

${ }^{16}$ Here we do not address the problem of initial conditions for the inflaton. Recently in [135] it has been suggested that in the case of DBI inflation this problem can be much less dramatic than for the canonical case.

${ }^{17}$ We stress that the term "relativistic" does not refer to GR effects. It is only used in analogy with special relativity, where, like for a DBI field, there is an upper bound for the speed. 
the DBI regime) of the potential where we can expand in $\phi / d$

$$
\gamma \simeq \frac{M_{P l} \phi}{\sqrt{6} d^{2}} \sqrt{f \Lambda} \quad \Rightarrow \quad \frac{\phi_{C M B}}{d} \lesssim \frac{\phi(\gamma=22)}{d} \simeq 22 \sqrt{6} \frac{d}{M_{P l} \sqrt{f \Lambda}} .
$$

Inflation ends when $\epsilon_{D B I} \gtrsim 1$, or equivalently when the condition (5.51) is no longer fulfilled and the kinetic energy becomes comparable to the potential energy. From figure 5.5 we see that $\epsilon_{D B I}$ becomes of order one for $\phi \rightarrow \pi d$. Expanding around $\phi=\pi d$ we get

$$
\frac{V^{3 / 2}}{\left|V^{\prime}\right| M_{P l}} \sqrt{3 f}=-\frac{d \sqrt{3 \Lambda f}\left(1+\cos \frac{\phi}{d}\right)^{3 / 2}}{M_{P l} \sin \frac{\phi}{d}} \simeq \sqrt{3 \Lambda f} \frac{(\phi-\pi d)^{2}}{2 \sqrt{2} M_{P l} d},
$$

which leads to the following analytical estimate (accurate for large $d \sqrt{\Lambda f} / M_{P l}$ ) for $\phi_{f}$

$$
\sqrt{3 \Lambda f} \frac{\left(\phi_{f}-\pi d\right)^{2}}{2 \sqrt{2} M_{P l} d}=1 \quad \Rightarrow \quad \phi_{f}=\pi d\left[1-\frac{2^{3 / 4} \sqrt{M_{P l}}}{\pi \sqrt{d}(3 f \Lambda)^{1 / 4}}\right] .
$$

Now that we know where the perturbations have to be produced, i.e. $\phi=\phi_{C M B}$, and where inflation ends, i.e. $\phi=\phi_{f}$, we can impose that the number of e-foldings ${ }^{18}$ in between is approximatively 60. From (5.54)

$$
60=N_{e}=\int_{\phi_{C M B}}^{\phi_{f}} \frac{H}{\dot{\phi}} d \phi \sim \frac{d}{M_{P l}} \sqrt{f \Lambda} .
$$

A consistency check is that the expansions we used in (5.56) and (5.57) are accurate for small $d / M_{P l}$ if we require $N_{e} \sim d \sqrt{f \Lambda} / M_{P l} \sim 60$.

The COBE normalization for the amplitude of the scalar perturbations gives us the constraint

$$
2 \times 10^{-9} \simeq \mathcal{P}_{R}=\frac{H^{4} f}{4 \pi^{2}} \quad \Rightarrow \quad \Lambda \sqrt{f} \simeq \frac{M_{P l}^{2}}{2000}
$$

The tensor modes are negligible: $\Delta \phi \sim \pi d<M_{P l}$. An upper bound is obtained estimating $r$ at $\phi(\gamma=20) / M_{P l} \simeq\left(d / M_{P l}\right)^{2}($ cf. $(5.56))$

$$
r \leq\left.\frac{16 \epsilon}{\gamma}\right|_{\phi(\gamma=20)} \simeq \frac{12}{f \Lambda}
$$

Summarizing, we can use the COBE normalization and the requirement of 60 e-foldings to express two of the three parameters of the model in terms of the third one:

$$
\{d, f, \Lambda\} \quad \longrightarrow \quad\left\{d, f=\frac{72^{2} 10^{10}}{d^{4}}, \Lambda=\frac{d^{2} M_{P l}^{2}}{12^{2} 10^{8}}\right\} .
$$

\footnotetext{
${ }^{18}$ As we already mentioned around (3.11), the number of e-foldings $N_{C M B}$ before the end of inflation when the CMB perturbations are produced can be different from 60 by some $30 \%$, e.g. depending on the reheating temperature, etc... For concreteness we take $N_{C M B}=60$, but another choice would not alter the conclusions of our analysis.
} 

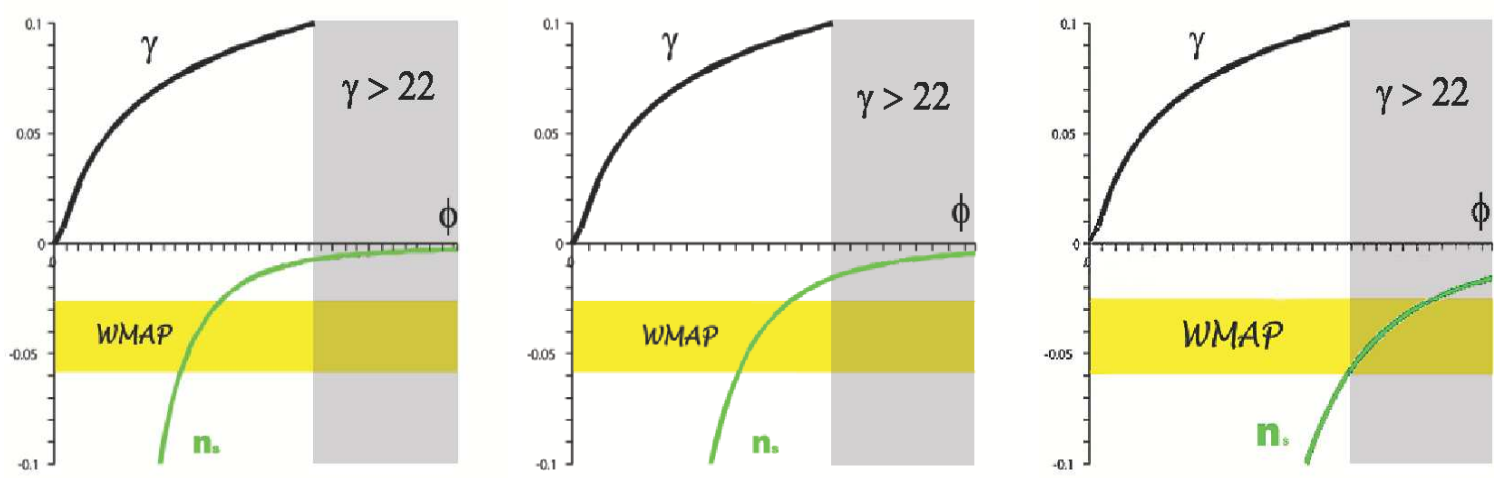

Figure 5.6: The scalar spectral index $n_{s}$ and $\gamma$ are plotted for three different values $d=$ $0.12,0.08,0.04$. The scale on the horizontal axis refers to $n_{s}$, while $\gamma$ is plotted in a logarithmic scale, where the maximum is $\gamma=22$. For $d<0.04$ the requirements $n_{s}=0.958 \pm 0.016$ and $\gamma<22$ can not be satisfied at the same time.

A constraint on $d$ comes from the spectral index of the scalar perturbations $n_{s}$. The analytical expression for $n_{s}$ is not so illuminating; we plot $n_{s}$ in figure 5.5.3 for various values of $d$. After imposing a precise value for the scalar spectral index, e.g. $n_{s}=0.958$, $\gamma$ just depends on $d$; hence detecting some non-Gaussianities would determine $d$ and completely fix the parameters of the model. As it is clear from figure 5.5.3, the two constraints $n_{s}=0.958 \pm 0.016$ and $\gamma<22$ lead to a lower bound on $d$. This can be estimated numerically as $d>0.04 M_{P l}$.

Thus one of the effects of the DBI kinetic term is to relax the bound $d>3.5 M_{P l}$ obtained in [103] to $d>0.04 M_{P l}$. This means that, provided one can arbitrarily choose $\Lambda$ and $f$, superplanckian values of $d$ are not required anymore ${ }^{19}$.

To finish this section we want to address the question whether it is possible to reproduce CMB data within the inflation at the tip model, i.e. if the consistency of string theory allows one to find stringy parameters that lead to the values in (5.61). The answer is negative: this embedding of DBI inflation in string theory is not phenomenologically viable. The reason is the following. From our discussion in section 5.5.1, we know that generically $d<M_{P l}$; from (5.58) it is clear that if we want to get 60 e-foldings we need $f \Lambda \gg 1$. As we show in detail in the next section, this implies that the energy density during inflation is bigger than the warped string scale and our supergravity approximation is inconsistent. As we will discuss in section 5.6.1 this problem can be overcome if one considers potentials that possesses both slow-roll flat regions and other regions where the DBI conditions (5.51) and (5.52) are satisfied.

${ }^{19} \mathrm{We}$ would like to stress that this statement is meant from an effective action point of view. In a concrete (e.g. string theory) model one should carefully consider the setup: the subplanckian (effective) parameter $d$ might arise from a superplanckian string parameter redshifted by the large warping. 


\subsection{A no-go result and how to evade it}

In this section we derive a no-go result for string theory DBI inflation along the tip, i.e. with constant warp factor. As for every no-go result the key point are the assumptions so let us clearly state them:

1. First, we assume that the D3-brane motion takes place along the angular direction at the tip of a deformed conifold. This is in contrast to the most studied case of chapter 4 where the D3-brane moves along the radial direction. Some of the motivations that led us to this choice are: the large mass generated by moduli stabilization (reviewed in section 4.3) affects the radial but not the angular brane inflation ${ }^{20}$; the warp factor $f$ varies in the radial direction but does not depend on the angular position and it is therefore a constant on the tip 21 . Finally, we notice that the field range for the angular motion at the tip is smaller than for the radial motion 22

2. Second, we assume that the inflaton motion is exclusively relativistic from the moment when the CMB perturbations are produced all the way until the end of inflation. In formulae

$$
\frac{V^{\prime 2}}{3 V} f M_{P l}^{2} \gg 1 \quad \Rightarrow \quad \gamma \gg 1
$$

This condition selects a class of potentials or equivalently a class of D7-embeddings $g(z)$ leading to these potentials. As we show in the following, potentials in this class do not give enough e-foldings of inflation at the tip. It is this assumption that we will relax in the next section where we will provide a simple example of a potential that possesses both DBI and slow-roll regions. We will argue that such potentials are promising candidates for a successful model of D-brane inflation.

The argument is now based on the impossibility to obtain enough e-foldings under these two assumptions. Consider

$$
\begin{aligned}
N_{e} & =\int d \phi \frac{H}{\dot{\phi}}=\int \frac{d \phi}{M_{P l}} \sqrt{\frac{V^{2}}{V^{\prime 2}}+\frac{f V M_{P l}^{2}}{3}} \\
& \simeq \int \frac{d \phi}{M_{P l}} \sqrt{\frac{f V}{3}} \lesssim \frac{\Delta \phi}{M_{P l}} \sqrt{\frac{f V_{\max }}{3}}
\end{aligned}
$$

${ }^{20}$ This does not mean that angular slow-roll inflation is automatic. Whether the slow-roll conditions are satisfied depends on the details of the potential (5.13)

${ }^{21} \mathrm{An}$ inflaton independent $f$ was already considered in [134, 136] but there it was obtained as an approximation of the radial motion of a D3-brane close to the tip of the deformed conifold where the AdS solution is not valid anymore.

${ }^{22}$ In view of the discussions in [137, 138, this implies that in these models only negligible tensor perturbations can be produced during slow-roll inflation. 

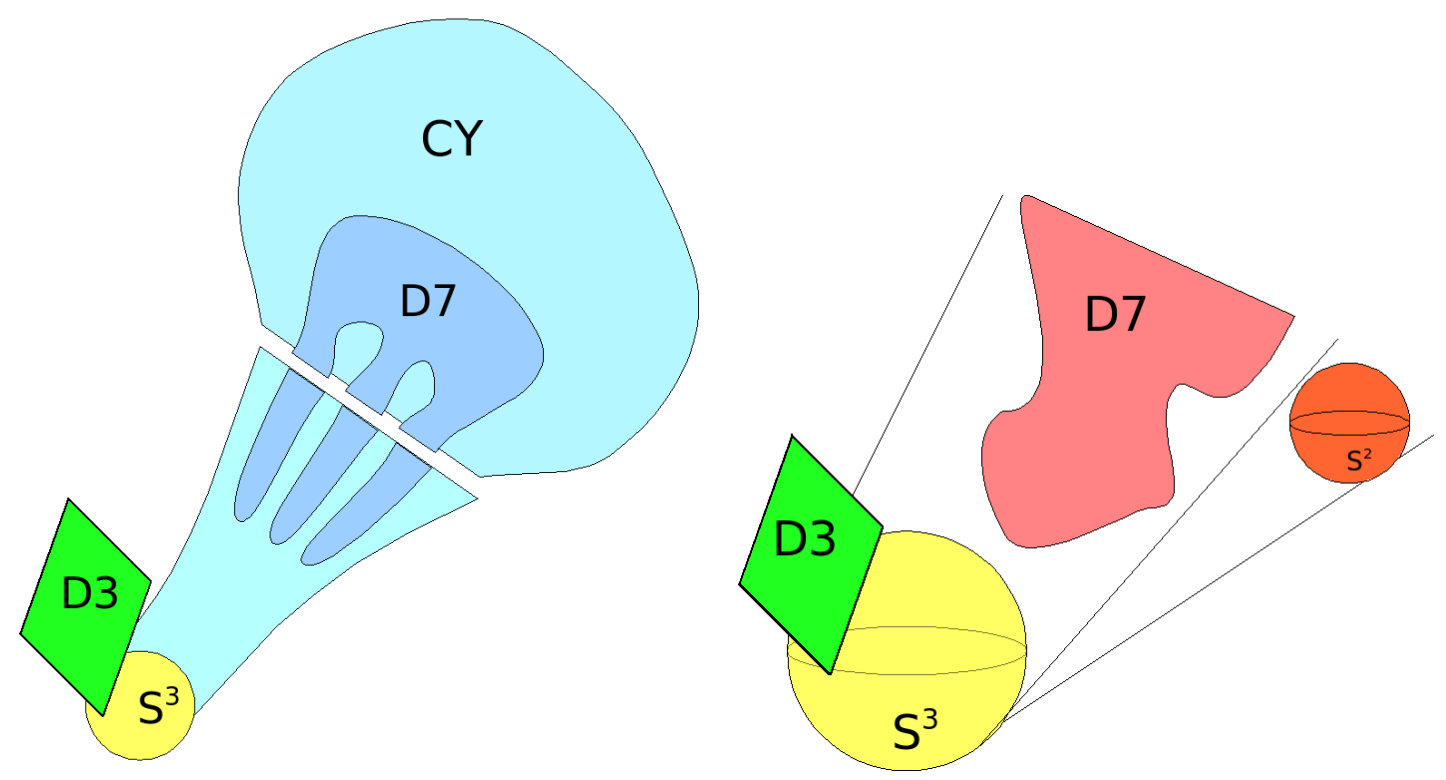

Figure 5.7: Different possibilities for inflation at the tip: on the left we draw the "threefold" D7 embedding in the warped throat; after gluing to a compact Calabi-Yau manifold the three 4-cycles are just parts of a single globally defined 4-cycle. On the right a possible generalization is depicted where instead of the simplest Kuperstein embedding, one considers a less symmetric one for the D7-branes.

where $\dot{\phi}$ and $H$ come from (5.48) and (5.49), respectively, and we have used assumption 2 to neglect the "slow-roll contribution" $V^{2} / V^{\prime 2}$ to $N_{e}$ (see e.g. (3.7)). To trust our effective action description of string theory we have to require that the energy density during inflation is much smaller than the redshifted string scale: $V_{\max } \ll M_{s}^{4} / h$. The parameter $f$ in 5.45 can be written in terms of stringy parameters as $h / T_{3}$. Hence

$$
\left\{\begin{array}{c}
V_{\max } \ll M_{s}^{4} / h \\
f=\frac{h}{T_{3}}=\frac{(2 \pi)^{3} g_{s} h}{M_{s}^{4}}
\end{array} \quad \Rightarrow \quad \sqrt{V_{\max } f} \ll \sqrt{\frac{(2 \pi)^{3} g_{s}}{3}} \simeq 9 \sqrt{g_{s}} .\right.
$$

The field variation $\Delta \phi$ during inflation is smaller than $\pi d$. As we argued at the end of section 5.5.1, generically $d \ll M_{P l}$ and therefore $\Delta \phi / M_{P l}<1$. In addition, we always assume $g_{s} \ll 1$. Putting these bounds together we conclude that it is impossible to have more than a few e-foldings from exclusively DBI inflation at the tip.

\subsubsection{Slow-roll-DBI alternation from generic embeddings}

A way to get around this negative result is to relax assumption 2, i.e. that during the whole 60 e-foldings of the observable inflation the motion is relativistic. Then in certain regions of the tip the potential can be of the slow-roll type and there the motion becomes 
non-relativistic; the "slow-roll term" $V^{2} / V^{\prime 2}$ can give a large contribution to $N_{e}$ in 5.62 increasing the number of e-foldings.

For a generic Kuperstein embedding defined by an arbitrary holomorphic function $\tilde{g}$, the potential was given in (5.11). The fact that Kuperstein embeddings preserve an $S O(2)$ symmetry implies that $V$ depends only on three real coordinates $\tau, x_{1}$ and $x_{23}$ (up to $S O(4)$ permutations). The volume can be fixed à la KKLT and we have to study $V\left(\tau_{0}\left(x_{1}, x_{23}\right), x_{1}, x_{23}\right)$.

As a result of our discussion, we should look for functions $\tilde{g}\left(z_{23}\right)$ such that the resulting scalar potential is of the slow-roll type somewhere on the tip. This can give us enough e-foldings avoiding the no-go theorem of the last section. Depending on the explicit form of $\tilde{g}$, the CMB perturbations could be produced during a DBI or a slow-roll phase. We leave a deeper investigation of this promising model for the future. Here we comment on an alternative possibility.

Consider the embedding

$$
g(z)=1-\frac{z_{1}^{3}}{\mu}
$$

where for simplicity we take $\mu$ to be real. In a non-compact conifold, (5.63) defines three disconnected 4-cycles, each one described by the embedding $z_{1}=e^{i 2 \pi j / 3} \mu^{1 / 3}$ for $j=0,1,2$, respectively. If a stack of D7-branes is wrapped around each 4-cycle the configuration is supersymmetric because all the three stacks respect the same supersymmetries as the background. We then suppose that it is possible to "cut" the conifold and "glue" it to a compact Calabi-Yau manifold; in addition, we ask that these three 4-cycles become part of a single 4-cycle. These are strong assumptions and we do not have anything to say about the hard problem of showing that the above construction can be actually realized. Here we would just like to present an interesting feature of this configuration.

In the global compact picture, there is just one stack of D7-branes that wraps a single 4-cycle. Gaugino condensation on these D7's can give rise to a superpotential that, for a D3-brane inside the throat, is well approximated by

$$
W=W_{0}+A_{0}\left(1-\frac{z_{1}^{3}}{\mu}\right)^{1 / n} e^{-a T} .
$$

The same expansion as discussed in section 5.4 leads to an effective potential of the form

$$
V \simeq \Lambda\left(1+\cos ^{3} \frac{\phi}{d}\right)
$$

that we plot in figure (5.8). The potential presents a flat inflection point where first and second derivatives vanish and slow-roll inflation can take place. 


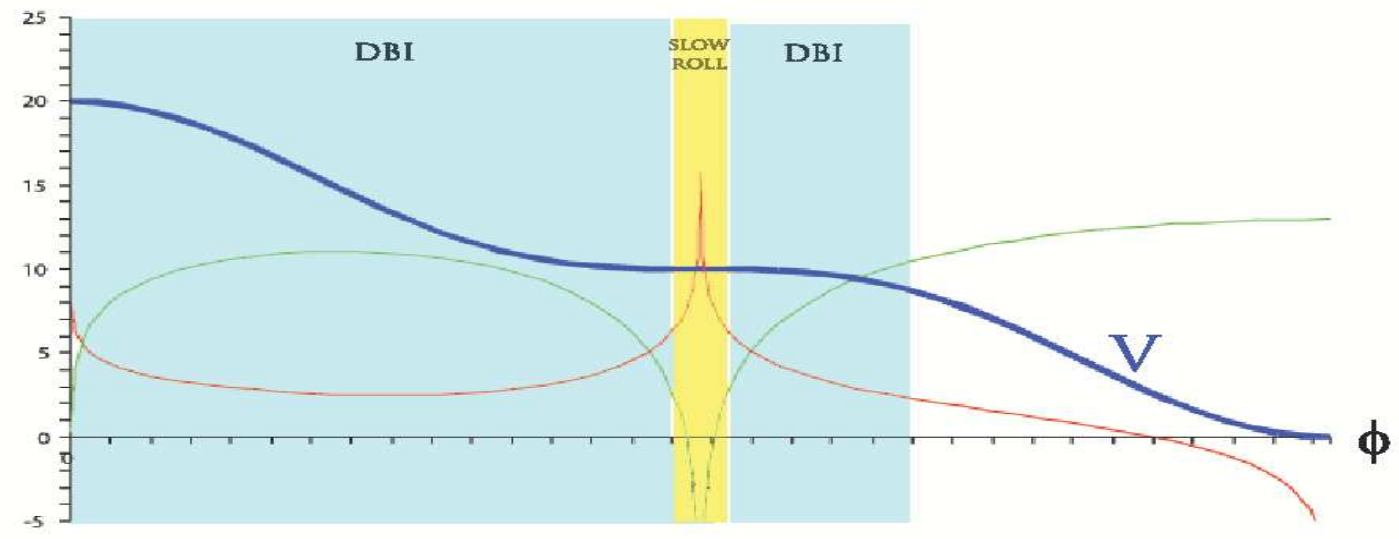

Figure 5.8: The thick (blue) line is the potential in (5.65). The two thin lines are the two DBI conditions (5.51) and (5.52) plotted in logarithmic scale. When they approach zero the DBI conditions are not satisfied anymore and the relativistic inflation regime ends. Around the inflection point first and second derivative of the potential are very small and slow-roll (non relativistic) inflation can take place. We highlight with different colors the DBI and slow-roll phases.

We already found the inflection point potential in chapter 4 . The discussion of its cosmological implications is analogous. If the first derivative at the inflection point is strictly vanishing, then the inflaton reaches it only in an infinite time and inflation lasts forever. There are two possibilities to regularize this divergence: as discussed in section 4.6.2 (see [64]), the initial speed of the inflaton (or equivalently corrections to the strict slow-roll regime) might allow it to pass the inflection point in a finite time. A second competing effect is given by the subleading terms that we neglected in the potential; they can have a non-vanishing first derivative at the inflection point. If this first derivative has the right sign, the total number of e-foldings is finite, otherwise a local minimum is formed as in figure 4.1 .

The CMB perturbations in this model can be produced either in the DBI or in the slow-roll phase. In the first case, the analysis is very similar to the one we performed in section 5.5.3. The slow-roll phase would then only provide enough e-foldings and would not have other observable consequences. In the second case, inflation takes place mainly in the slow-roll regime, except for the last few unobservable e-foldings of DBI phase (on the right hand side of figure (5.8)). The analysis is then very similar to other cases of inflection point inflation in the literature [64, 68, 139, 140]. The phenomenological predictions are very sensitive to the parameters, e.g. to those determining the first derivative of the potential at the inflection point.

Summarizing, inflation at the tip is a promising candidate for a phenomenologically successful embedding of DBI inflation in string theory. The right perturbations can easily be 
produced; to obtain enough e-foldings it is required that, in some region, the potential is slow-roll flat. Above, we have given a (semi) explicit example, but we expect that many others can be found studying more generic D7-embeddings (e.g. with a generic $\tilde{g}$ in (4.5)).

\subsection{A summary on angular brane inflation}

In this chapter we have proposed and studied a model of D3-brane inflation that takes place along the angular directions of a warped conifold and where no anti D3-branes are needed. A D3-brane moves on the tip of a warped deformed conifold [107] embedded in a compact Calabi-Yau manifold, which is an $S^{3}$ (see figure 5.1). The potential comes from the F-term where the threshold corrections to the non-perturbative superpotential are taken into account. Inflation at the tip is an explicit example of how several issues of brane inflation can be addressed in a way different from the standard radial case that we studied in chapter 4

We have looked for inflationary trajectories, considering two classes:

1. Slow-roll inflation: it is possible to achieve but only allowing for fine tuning. In a small region of the stringy parameter space (where $\Lambda \simeq 2 C$ ) the inflaton potential becomes very flat on the top (see figure 5.4). This is a kind of hilltop model [105, 106], i.e. of the form

$$
V \simeq \Lambda-\frac{1}{2} m^{2} \phi^{2}-\lambda \phi^{4}
$$

The negative squared mass of the inflaton can be made small by fine tuning, and we obtain a string theory model of slow-roll inflation in perfect agreement with $C M B$ data (see chapter 3 and section 5.5.2). When the mass term $m^{2} \phi^{2}$ is negligible with respect to the $\lambda \phi^{4}$ term, the model predicts $n_{s} \simeq 0.94$, negligible tensor modes and the scale of inflation $(\Lambda)^{1 / 4} \sim d \times 10^{-3}$, where $d$ was defined in $(5.29)$.

2. DBI inflation: as the inflaton corresponds to the position of a D3-brane, the kinetic term comes from the DBI action. This can allow inflation to take place even with a steep potential [50, 51]. We investigated this possibility and discovered that, although perturbations compatible with $C M B$ data can be produced, the DBI regime can never lead to more than a few e-foldings. We argued that more general embeddings than the one we studied here (Kuperstein embedding [73] with constang $\tilde{g}$ ) can possess flat regions that would increase the number of e-foldings and lead to a successful model. We leave a more detailed study of this interesting possibility for future investigation.

During the study of both radial (chapter 4) and angular (present chapter) inflation, we have neglected perturbative quantum corrections. These can arise in different ways: there 
are $\alpha^{\prime}$ corrections, suppressed by powers of the string scale $M_{s}=\left(\alpha^{\prime}\right)^{-1 / 2}$, which are induced by the higher oscillation modes of the string that we have ignored by looking at the massless spectrum (see chapter 2). There are field theory and/or string loop corrections suppressed by the appropriated coupling. There is the tower of KK modes and the effects due to the large warping. For a completely successful string inflation model, all these effects have to be carefully taken into account. This is a formidable challenge and we are still far from that goal.

On the other hand it is very important to check the consistency of our effective description, to know if string theory and/or quantum corrections can change the qualitative features of a certain model. This is why we will dedicate the next chapter to this issue. We will consider an explicit model and carefully analyze the effects of several types of corrections. On the other hand, we will abandon the subject of string cosmology and switch to string particle phenomenology. Particle physics is the other wide field of research, where by general considerations (see chapter 1) string theory can be applied most usefully. We postpone a further discussion to the next chapter.

Finally, in the following we list some interesting directions for future research

- The case of a generic embedding (with a generic $\tilde{g}$ in (4.5) or those discussed in [72]) has to be studied in detail looking for an explicit working model. A criteria to keep in mind in this search follows from our no go result in section (5.6): a successful model needs to have a potential with at least one slow-roll flat region.

- The absence of an anti D3-brane makes the mechanism of graceful exit and reheating in the present model very different from the standard brane-anti brane inflation model. A mechanism naturally embedded in the model is the D-brane trapping (which was one of the motivations of the original proposal of brane inflation [49]). In 93 it was shown that in the collision of branes, the kinetic energy can be transferred to the gauge fields living on the newly created stack. This would create a thermal bath in the world volume of the branes that could evolve into our universe. It would be interesting to investigate quantitatively the phenomenological viability of this idea.

- It would be desirable to develop a gauge dual description of the brane inflation at the tip proposed in this paper, a work in this direction is [141] (see also [142]). Although there the origin of the potential is different, the authors provide a gauge theory description of the radial motion of a brane in a resolved warped deformed conifold. 


\section{On soft terms from large volume compactifications}

As we have seen in chapter 2, the KKLT strategy [8, 20] for producing stabilized string vacua of type IIB, can serve as a starting point for phenomenological constructions. In chapters 4 and 5 for example, we have studied two models of brane inflation in this setup. Here we will focus on a generalization of the KKLT setup, known as the "large volume scenario" (LVS for short, we introduce it in section 6.2.3), where many phenomenological issues have been addressed, such as for example soft supersymmetry breaking [143, 144, 145], the QCD axion [146, 147], neutrino masses [148], first attempts at LHC phenomenology [149], and where also some closed string inflationary models have been constructed [58, 150, 151, 152].

Although tantalizing, the models discussed in the aforementioned papers as well as the inflationary models of chapters 4 and 5 raise many questions. It remains an open problem to construct complete KKLT models in string theory, as opposed to supergravity. Problems one faces include things like the description of RR fluxes in string theory, showing that the necessary nonperturbative effects actually can and do appear in a way consistent with other contributions to the potential (for progress in this direction, see [61, 153, 154, 23, 155, 156, 157, 158, 159, 160, 161, 162, 114, 163, 164, 165, 166]), and verifying that one can uplift to a Minkowski or deSitter vacuum without ruining stabilization [22, 167, 168, 161, 125]. These issues become possibly even more important in some extensions of KKLT and in particular in LVS [169, 143], where corrections to the tree-level supergravity effective action (computed in [170]) play a significant role, and where the compactification volume can be stabilized as large as $10^{15}$ in string units. In LVS, since string corrections play a crucial role, striving for actual string constructions seems quite important. In the end, the restrictiveness this entails may greatly improve predictivity, or kill the models completely as string compactifications.

In this chapter, we will not improve on the consistency of KKLT or LVS in general, but rather assume the existence of LVS models in string theory, and then perform selfconsistency checks. This is a modest step on the way towards reconciling phenomenologically promising scenarios with underlying string models. We will see that although a priori the situation looks very bleak, and one might have hastily concluded that even our modest consistency check would put very strong constraints on LVS, things are more interesting. It turns out that LVS jumps through every hoop we present it with, and instead of broad qualitative changes, we find only small quantitative changes. 
The structure of this chapter is as follows: we start in section 6.1 with some preliminaries; in section 6.2 we critically review the KKLT construction of section 2.6 and introduce the LVS; in section 6.3 we argue which kind of loop corrections we expect in LVS and compute their effects on the potential in an explicit example. In section 6.4 we compute the gaugino masses in LVS and comment on other susy-breaking terms. In section 6.5 we extend our discussion of string loop corrections to other classes of Calabi-Yau manifolds. We conclude in section 6.6 with a discussion of further corrections and a summary. A series of technical details are left to appendix $\mathrm{D}$.

\subsection{Preliminaries}

The main difference between KKLT and LVS is that LVS includes a specific string $\alpha^{\prime}$ correction $\Delta K_{\alpha^{\prime}}$ in the Kähler potential $K$ of the 4-dimensional $\mathcal{N}=1$ effective supergravity. Naturally, the 4-dimensional string effective action also contains other string corrections. Here, we will focus on $g_{\mathrm{s}}$ corrections due to sources (D-branes and O-planes). For some $\mathcal{N}=1$ and $\mathcal{N}=2$ toroidal orientifolds, these corrections were computed in [171] (see also [172]; for a comprehensive introduction to orientifolds, see [173]). Compared to the $\alpha^{\prime}$ correction $\Delta K_{\alpha^{\prime}}$ considered in LVS, the $g_{\mathrm{s}}$ corrections to the Kähler potential $\Delta K_{g_{\mathrm{s}}}$ will scale as

$$
\left(\Delta K_{\alpha^{\prime}}: \Delta K_{g_{\mathrm{s}}}\right) \sim\left(\mathcal{O}\left(\alpha^{\prime 3}\right): \mathcal{O}\left(g_{\mathrm{s}}^{2} \alpha^{\prime 2}\right)\right) \quad \text { (string frame) } .
$$

By naive dimensional analysis, one would expect that in a $1 / \mathcal{V}$ expansion, where $\mathcal{V}$ is the overall volume in the Einstein frame, 6.1) implies

$$
\Delta K_{\alpha^{\prime}} \sim \mathcal{O}\left(g_{\mathrm{s}}^{-3 / 2} \mathcal{V}^{-1}\right) \quad, \quad \Delta K_{g_{\mathrm{s}}} \sim \mathcal{O}\left(g_{\mathrm{s}} \mathcal{V}^{-2 / 3}\right) \quad \text { (Einstein frame) }
$$

If, contrary to what we assumed in chapter 4 and 5 , there is more than one Kähler modulus various combinations of Kähler moduli may appear in $\Delta K_{g_{\mathrm{s}}}$ in 6.2 , and a priori this could lead to even weaker suppression in $1 / \mathcal{V}$ than that shown. However, we will argue that $(6.2)$ is actually correct as far as the suppression factors in the $1 / \mathcal{V}$ expansion go. Nevertheless, even the suppression displayed in 6.2 seems to be a challenge for LVS, if indeed $\mathcal{V} \sim 10^{15}$. For $\mathcal{V}$ this large, $\Delta K_{g_{\mathrm{s}}}$ would dominate $\Delta K_{\alpha^{\prime}}$, since we do not expect the string coupling $g_{\mathrm{s}}$ to be stabilized extremely small. On the other hand, if we are interested in the effects $g_{\mathrm{s}}$ corrections may have on the existence of the large volume minima, the relevant quantity to look at is the scalar potential $V$, rather than the Kähler potential $K$. It turns out that certain cancellations in the expression for the scalar potential leave us with leading correction terms to $V$ that scale as

$$
\Delta V_{\alpha^{\prime}} \sim \mathcal{O}\left(g_{\mathrm{s}}^{-1 / 2} \mathcal{V}^{-3}\right) \quad, \quad \Delta V_{g_{\mathrm{s}}} \sim \mathcal{O}\left(g_{\mathrm{s}} \mathcal{V}^{-3}\right)
$$

This is already much better news for LVS. However, restoring numerical factors in 6.3), and with $g_{\mathrm{s}}$ typically not stabilized extremely small, it would seem that $\Delta K_{g_{\mathrm{s}}}$ could still 
have a significant effect both on stabilization and on the resultant phenomenology (like soft supersymmetry breaking terms, which also depend on the Kähler potential). We will see that although this is indeed so in principle, in practice the models we consider are surprisingly robust against the inclusion of $\Delta K_{g_{\mathrm{s}}}$. The clearest example of this is the calculation of gaugino masses in section 6.4. The result is that for the "11169 model" (analyzed in [144]), the correction to the gaugino masses due to $\Delta K_{g_{\mathrm{s}}}$ is negligible. Thus, for the most part, LVS survives our onslaught unscathed.

We consider this a sign that scenarios such as LVS deserve to be taken seriously as goals to be studied in detail in string theory, even as the caveats above (that apply to any KKLT-like setup) serve to remind us that there is much work left to be done to really understand phenomenologically viable stabilized flux compactifications in string theory.

\subsection{Review}

Let us begin by critically reviewing the KKLT setup we introduced in section 2.6. Then we generalize it by introducing the large volume scenarios. For reasons that will become clear and as opposed to chapter 4 and 5, we want now to allow for more than a single Kähler modulus.

\subsubsection{Back to KKLT}

The KKLT setup [8, 20], that we introduced in section 2.6, is a warped type IIB flux compactification on a Calabi-Yau (or more generally, F-theory) orientifold, with all moduli stabilized. In this chapter, we will neglect warping ${ }^{1}$.

In the 4-dimensional $\mathcal{N}=1$ effective supergravity, the Kähler potential and superpotential (see the end of section 2.5.1) read

$$
\begin{aligned}
K & =-\ln (S+\bar{S})-2 \ln (\mathcal{V})+K_{\mathrm{cs}}(u, \bar{u}), \\
W & =W_{\text {tree }}+W_{\mathrm{np}}=W(S, u)+\sum_{i} A_{i}(S, u) e^{-a_{i} T_{i}},
\end{aligned}
$$

where the volume $\mathcal{V}$ is a function of the Kähler moduli $T_{i}=\tau_{i}+i b_{i}$ whose real parts are 4 -cycle volumes and whose imaginary parts are axions $b_{i}$, arising from the integral of the RR 4-form over the corresponding 4-cycles; $u$ denotes generically all the complex structure moduli. In particular, the volume $\mathcal{V}$ depends on the $T_{i}$ only through the real parts $\tau_{i}$,

$$
\mathcal{V}=\mathcal{V}\left(T_{i}+\bar{T}_{i}\right)=\mathcal{V}\left(\tau_{i}\right)
$$

\footnotetext{
${ }^{1}$ Warping, on the contrary, was a crucial ingredient in the cosmological applications of chapter 4 and 5). For progress towards taking warping into account in phenomenological contexts, see [174, 175].
} 
and $A_{i}$ in the nonperturbative superpotential $W_{\mathrm{np}}$ a priori depends on the complexified dilaton $S$ and the complex structure moduli $u$ (notice that, contrary to the previous chapters, we do not consider any open string sector). After stabilization of $S$ and $u$ (demanding the supersymmetry conditions $D_{u} W=0=D_{S} W$ ), we have

$$
\begin{aligned}
K & =-\ln (S+\bar{S})-2 \ln (\mathcal{V})+K_{\mathrm{cs}}(u, \bar{u}) \\
W & =W_{\text {tree }}+W_{\mathrm{np}}=W_{0}+\sum_{i} A_{i} e^{-a_{i} T_{i}}
\end{aligned}
$$

Contrary to the previous chapters, we keep the dependence on the complexified dilaton $S$ and the complex structure moduli $u$ in the Kähler potential for now. Before we neglected this dependence since the Kähler metric obtained from (6.6) is block diagonal in the various moduli sectors (see also appendix D.6). In this chapter we will eventually take into account corrections that break this diagonal structure; then the inverse of the Kähler metric appearing in the F-term potential

$$
V=e^{K}\left(G^{\bar{J} I} D_{\bar{J}} \bar{W} D_{I} W-3|W|^{2}\right)
$$

will include some $S$ and $u$ dependence ${ }^{2}$. The scalar potential $V$ has a supersymmetric AdS minimum (see e.g. figure 2.4) at a radius that is barely large enough to make the use of a large-radius effective supergravity self-consistent, typically $\tau \sim 100$ (recall that $\tau$ has units of (length $\left.)^{4}\right) !^{3}$ In addition, to obtain a supersymmetric minimum at all, one needs to tune the flux superpotential $W_{0}$ to values exponentially small in $a \tau$, see e.g. (2.37). That is, the stabilization only works for a small parameter range. This is easy to understand, since we are balancing a nonperturbative term against a tree-level term. Let us briefly digress on the reasons for and implications of this balancing.

\subsubsection{Consistency of KKLT}

In the previous section we only considered the lowest-order supergravity effective action. As was already noted in the original KKLT paper, $\alpha^{\prime}$ corrections and $g_{\mathrm{s}}$ corrections (string loops) that appear in addition to the tree-level effective action could in principle affect stabilization. Oftentimes, the logic of string effective actions is that if one such correction matters, they all do, so no reliable physics can be learned from considering the first few corrections. If this is true, one can only consider regimes in which all corrections are suppressed. This is not necessarily so if some symmetry prevents the tree-level contribution to the effective action from appearing, so that the first correction (be it $\alpha^{\prime}$ or $g_{s}$ ) constitutes lowest order. This indeed happens for type IIB flux compactifications. As we saw section

\footnotetext{
${ }^{2}$ Anyways, we still assume that $u$ and $S$ are stabilized by fluxes at an higher scale, therefore in 6.7), the indeces $I$ and $J$ run only over the Kähler moduli.

${ }^{3}$ As we have already seen, e.g. in section 2.6, this minimum then has to be uplifted to dS or Minkowski by an additional contribution to the potential.
} 
2.5.1 and explicitely in (4.3.2), given the tree level Kähler potential (6.6), if we were to set $W_{\mathrm{np}}=0$, the remaining $K$ and $W$ in $(6.6)$ produce a no-scale potential, i.e. the scalar potential for the Kähler moduli then vanishes [176]. In KKLT, this no-scale structure is only broken by the nonperturbative contribution to the superpotential $W_{\mathrm{np}}$. Since each term in $W_{\text {np }}$ is exponentially suppressed in some Kähler modulus, the resulting terms in the potential are also exponentially suppressed. For instance, for the simpler example of a single modulus $\tau$, the potential (after already fixing the axionic partner as in section 4.4.1) reads

$$
\frac{V}{e^{K}}=\left[4|A|^{2} a \tau e^{-a \tau}\left(\frac{1}{3} a \tau+1\right)-4 a \tau\left|A W_{0}\right|\right] e^{-a \tau},
$$

meaning that even for moderate values of the Kähler modulus $\tau$, all these terms are numerically very small. Corrections in $\alpha^{\prime}$ and $g_{s}$, however, are expected to go as powers of Kähler moduli $\tau$, so will dominate the scalar potential for most of parameter space. In particular, it was argued in [169, 143] that only for very small values of $W_{0}$ can perturbative corrections to the Kähler potential be neglected. It was the insight of [169] that even if $W_{0}$ is $\mathcal{O}(1)$ (which is more generic than the tiny value for $W_{0}$ required in KKLT), there can still be a competition between the perturbative and nonperturbative corrections to the potential in regions of the Kähler cone where large hierarchies between the Kähler moduli are present. We now review this scenario.

\subsubsection{Large volume scenario (LVS)}

As was shown in [170], the no-scale structure (and factorization of moduli space) is broken by perturbative $\alpha^{\prime}$ corrections to the Kähler potential, such as

$$
K=-\ln \left(2 S_{1}\right)-2 \ln \left(\mathcal{V}+\frac{1}{2} \xi S_{1}^{3 / 2}\right)+K_{\mathrm{cs}}(u, \bar{u}),
$$

where $\xi=-\zeta(3) \chi / 2(2 \pi)^{3}$ and $S_{1}=\operatorname{Re} S$. For large volume $\mathcal{V}$, we see that the perturbative correction goes as a power in the volume,

$$
-2 \ln \left(\mathcal{V}+\frac{1}{2} \xi S_{1}^{3 / 2}\right)=-2 \ln \mathcal{V}-\frac{\xi S_{1}^{3 / 2}}{\mathcal{V}}+\ldots
$$

which by the discussion in the previous subsection will dominate in the scalar potential if all Kähler moduli are even moderately large. Using the superpotential

$$
W=W_{0}+W_{\mathrm{np}}=W_{0}+\sum_{i} A_{i} e^{-a_{i} T_{i}},
$$

the scalar potential has the structure

$$
\begin{aligned}
V= & V_{\mathrm{np} 1}+V_{\mathrm{np} 2}+V_{3} \\
= & e^{K}\left\{G^{\bar{\jmath} i} \partial_{\bar{\jmath}} \bar{W}_{\mathrm{np}} \partial_{i} W_{\mathrm{np}}+\left[G^{\bar{\jmath} i} K_{\bar{\jmath}}\left(\bar{W}_{0}+\bar{W}_{\mathrm{np}}\right) \partial_{i} W_{\mathrm{np}}+\text { c.c. }\right]\right. \\
& \left.+\left(G^{\bar{\jmath} i} K_{\bar{\jmath}} K_{i}-3\right)|W|^{2}\right\} .
\end{aligned}
$$

\footnotetext{
${ }^{4}$ Here $\xi$ differs by a factor $(2 \pi)^{-3}$ from [170] because we use the string length $l_{s}=2 \pi \sqrt{\alpha^{\prime}}$.
} 
For concrete calculations we will use the model based on the hypersurface of degree 18 in $\mathbb{P}_{[1,1,1,6,9]}^{4}$ (see [177, 61, 178] for background information on its topology. Some comments about generalizations to other models with arbitrary numbers of Kähler moduli are given in appendix D.1.2. The defining equation is

$$
z_{1}^{18}+z_{2}^{18}+z_{3}^{18}+z_{4}^{3}+z_{5}^{2}-18 \psi z_{1} z_{2} z_{3} z_{4} z_{5}-3 \phi z_{1}^{6} z_{2}^{6} z_{3}^{6}=0
$$

and it has the Hodge numbers $h^{1,1}=2$ and $h^{2,1}=272$ (only two of the complex structure moduli $\psi$ and $\phi$ have been made explicit in (6.13); moreover, not all of the 272 survive orientifolding). We denote the two Kähler moduli by $T_{b}=\tau_{b}+i b_{b}$ and $T_{s}=\tau_{s}+i b_{s}$, where $\tau_{b}$ and $\tau_{s}$ are the volumes of 4-cycles, and the subscripts " $b$ " and " $s$ " are chosen in anticipation of the fact that one of the Kähler moduli $\left(\tau_{b}\right)$ will be stabilized big, and the other one $\left(\tau_{s}\right)$ will be stabilized small. An interesting property of this model is that it allows expressing the 2-cycle volumes $t_{i}$ explicitly as functions of the 4-cycle volumes $\tau_{j}$, so that the total volume of the manifold can be written directly in terms of 4-cycle volumes, yielding

$$
\begin{gathered}
\mathcal{V}=\frac{1}{9 \sqrt{2}}\left(\tau_{b}^{3 / 2}-\tau_{s}^{3 / 2}\right), \\
\tau_{b}=\frac{\left(t_{s}+6 t_{b}\right)^{2}}{2}, \quad \tau_{s}=\frac{t_{s}^{2}}{2} .
\end{gathered}
$$

Following [143], we are interested in minima of the potential with the peculiar property that one Kähler modulus $\tau_{b} \sim \mathcal{V}^{2 / 3}$ is stabilized large and the rest are relatively small (but still large compared to the string scale),

$$
a \tau_{s} \sim \ln \mathcal{V} \sim \frac{3}{2} \ln \tau_{b}
$$

in the case at hand. Thus, we expand the potential around large volume, treating $e^{-a \tau_{s}}$ as being of the same order as $\mathcal{V}^{-1}$. In the end one has to check that the resulting potential indeed leads to a minimum consistent with the exponential hierarchy $a \tau_{s} \sim \ln \mathcal{V}$, so that the procedure is self-consistent. Applying this strategy, the scalar potential at leading order in $1 / \mathcal{V}$ becomes $5^{5}$

$$
V_{\mathcal{O}\left(1 / \mathcal{V}^{3}\right)}=\left(\frac{12 \sqrt{2}|A|^{2} a^{2} \sqrt{\tau_{s}} e^{-2 a \tau_{s}}}{\mathcal{V} S_{1}}-\frac{2 a\left|A W_{0}\right| \tau_{s} e^{-a \tau_{s}}}{\mathcal{V}^{2} S_{1}}+\xi \frac{3\left|W_{0}\right|^{2} \sqrt{S_{1}}}{8 \mathcal{V}^{3}}\right) e^{K_{\mathrm{cs}}} .
$$

From here one can see the existence of the large volume minima rather generally. By the Dine-Seiberg argument [179], the scalar potential goes to zero asymptotically in every

\footnotetext{
${ }^{5}$ Here we have already stabilized the axion $b_{s}$, i.e. solved $\partial V / \partial b_{s}=0$, which produces the minus sign in the second term; this is also true with many small moduli $\tau_{i}$. See appendix D.1.2 for details. Also note that solving $D_{u} W=0=D_{S} W$ causes the values of $u$ and $S$ at the minimum to depend on the Kähler moduli. However, this dependence arises either from the nonperturbative terms in the superpotential or from the $\alpha^{\prime}$-correction to the Kähler potential. Thus it would only modify the potential at subleading order in the $1 / \mathcal{V}$ expansion.
} 
direction. Along the direction (6.15), for large volume the leading term in $(6.16)$ is

$$
V \sim V_{\mathrm{np} 2} \propto-\frac{\ln \mathcal{V}}{\mathcal{V}^{3}}
$$

which is negative, so the potential $V$ approaches zero from below. For moderately small values of the volume, $V$ is positive (this is guaranteed if the Euler number $\chi$ is negative, hence $\xi$ positive), so in between there is a minimum. This minimum is typically nonsupersymmetric, and because we are no longer balancing a tree-level versus a nonperturbative term, we can find minima at large volume - hence the name large volume scenario (LVS) ${ }^{6}$ To be precise, in flux compactifications we move in parameter space by the choice of discrete fluxes, but since $\mathcal{V}$ is exponentially sensitive to parameters like $S_{1}$, large volume minima appear easy to achieve also by small changes in flux parameters. If we allow for very small values of $W_{0}$ (so that KKLT minima exist at all), the above minimum can coexist with the KKLT minimum [180, 143]. Here, we will allow $W_{0}$ to take generic values of order one.

The astute reader will have noticed that this argument for the existence of the LVS minimum is "one dimensional", as it only takes into account the behavior of the potential along the direction (6.15). One must of course check minimization with respect to all Kähler moduli. In [169] a plausibility argument to this effect was given, and the existence of the minimum was explicitly checked in the case of the $\mathbb{P}_{[1,1,1,6,9]}^{4}$ model by explicitly minimizing the potential 6.16 with respect to the Kähler moduli. In doing so, it is convenient to trade the two independent variables $\left\{\tau_{b}, \tau_{s}\right\}$ for $\left\{\mathcal{V}, \tau_{s}\right\}$ so that $\partial_{\tau_{s}} \mathcal{V}=0$, as then the last term in (6.16) is independent of $\tau_{s}$ (this will be different when we include loop corrections). Extremizing with respect to $\tau_{s}$, and defining

$$
X \equiv A e^{-a \tau_{s}}
$$

one obtains a quadratic equation for $X$,

$$
0=\frac{\partial V}{\partial \tau_{s}}=\left(-\frac{6 \sqrt{2} a^{2}}{\sqrt{\tau_{s}} S_{1} \mathcal{V}}\left(4 a \tau_{s}-1\right) X^{2}+\frac{2 a\left|W_{0}\right|}{S_{1} \mathcal{V}^{2}}\left(a \tau_{s}-1\right) X\right) e^{K_{\mathrm{cs}}} .
$$

In (6.18), we chose $A$ to be real as a potential phase can be absorbed into a shift of the axion $b$ and disappears after minimization with respect to $b$ (see section D.1.2 and 4.4.1). Two comments are in order. The quadratic equation (6.19) has just one meaningful solution $\left(X=0\right.$ corresponds to $\left.\tau_{s}=\infty\right)$. Moreover, when expanding (6.19) in $1 /\left(a \tau_{s}\right)$, the leading terms arise from derivatives of the exponential.

Formula 6.19) is an implicit equation determining $\tau_{\mathrm{s}}$. However, one can easily solve (6.19) for $X$ and obtains

$$
X=A e^{-a \tau_{s}}=\frac{\sqrt{2}\left|W_{0}\right|}{24 a \mathcal{V}} \sqrt{\tau_{s}}\left(1-\frac{3}{4 a \tau_{s}}\right)+\mathcal{O}\left(\frac{1}{\left(a \tau_{s}\right)^{2}}\right) .
$$

\footnotetext{
${ }^{6}$ By "tree-level" we intend "tree-level supergravity", i.e. for the purposes of this chapter we call both $\alpha^{\prime}$ and $g_{s}$ corrections "quantum corrections".
} 
The hierarchy (6.15) is obvious in this solution, rendering the procedure self-consistent. One also notices that reasonably large values of $\tau_{\mathrm{s}}$ (e.g. 35) are not difficult to obtain, if $\mathcal{V}$ is stabilized large enough; for example, simply set $a \sim 1, A \sim 1, W_{0} \sim 1$. We fill in the numerical details, following [169], in appendix D.1.1 (including some further observations).

\subsection{String loop corrections to LVS}

As already emphasized, the $\alpha^{\prime}$ correction proportional to $\xi$ is only one among many corrections in the string effective action. We now consider the effect of string loop corrections on this scenario and what the regime of validity is for including or neglecting those corrections. Volume stabilization with string loop corrections but without nonperturbative effects was considered in [91].

To be precise, the corrections considered in [91 were those of [171], that were computed for toroidal $\mathcal{N}=1$ and $\mathcal{N}=2$ orientifolds. Here, we would need the analogous corrections for smooth Calabi-Yau orientifolds. Needless to say, these are not known. Faced with the fact that the string coupling $g_{\mathrm{s}}$ is stabilized at a finite (and typically not terribly small) value, we propose that attempting to estimate the corrections based on experience with the toroidal case is better than arbitrarily discarding them. As we will see, if our estimates are correct, typically the loop corrections can be neglected, though there may at least be some regions of parameter space where they must be taken into account (see figure 6.4). (In section 6.5, we will briefly consider "cousins" of LVS where they can not be neglected anywhere in parameter space.) Improvement on our guesswork would of course be very desirable.

\subsubsection{From toroidal orientifolds to Calabi-Yau manifolds}

We would like to make an educated guess for the possible form of one-loop corrections in a general Calabi-Yau orientifold. All we can hope to guess is the scaling of these corrections with the Kähler moduli $T$ and the dilaton $S$. The dependence on other moduli, like the complex structure moduli $u$, can not be determined by the following arguments (even in the toroidal orientifolds this dependence was quite complicated).

In order to generalize the results of [171] to the case of smooth Calabi-Yau manifolds, we should first review them and in particular remind ourselves where the various corrections come from in the case of toroidal orientifolds. There, the Kähler potential looks as follows 
(we will explain the notation as we go along):

$$
\begin{aligned}
K=-\ln \left(2 S_{1}\right)- & 2 \ln (\mathcal{V})+K_{\mathrm{cs}}(u, \bar{u})-\frac{\xi S_{1}^{3 / 2}}{\mathcal{V}} \\
+ & \sum_{i=1}^{3} \frac{\mathcal{E}_{i}^{K}(u, \bar{u})}{4 \tau_{i} S_{1}}+\sum_{i \neq j \neq k}^{3} \frac{\mathcal{E}_{k}^{W}(u, \bar{u})}{4 \tau_{i} \tau_{j}} .
\end{aligned}
$$

There are two kinds of corrections. One comes from the exchange of Kaluza-Klein (KK) modes between D7-branes (or O7-planes) and D3-branes (or O3-planes, both localized in the internal space), which are usually needed for tadpole cancellation, cf. fig. 6.1. This

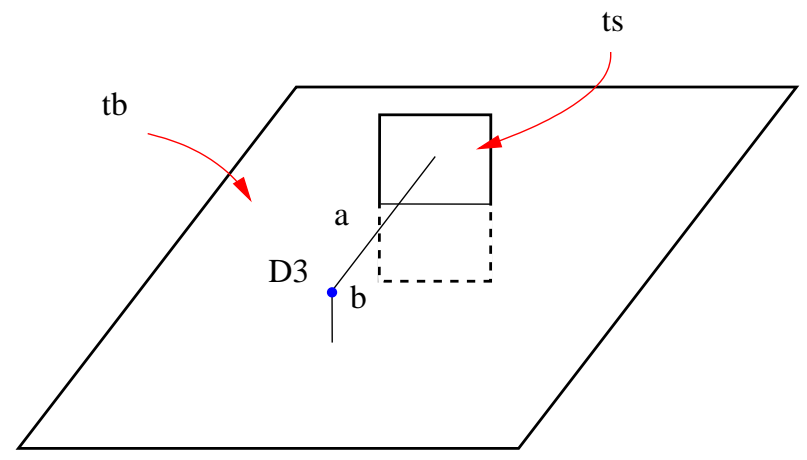

Figure 6.1: The loop correction $\mathcal{E}^{K}$ comes from the exchange of closed strings, or equivalently an open-string one-loop diagram, between the D3-brane and D7-branes (or O7-planes) wrapped on either the small 4-cycle $\tau_{\mathrm{s}}$ (as in $a$ ) or the large 4-cycle $\tau_{\mathrm{b}}$ (as in $b$ ). The exchanged closed strings carry Kaluza-Klein momentum.

leads to the first kind of corrections in 6.21), proportional to $\mathcal{E}_{i}^{K}$ where the superscript $(K)$ reminds us that these terms originate from KK modes. In the toroidal orientifold case, this type of correction is suppressed by the dilaton and a single Kähler modulus $\tau_{i}$, related to the volume of the 4-cycle wrapped by the D7-branes (or O7-planes, respectively).7 We expect an analog of these terms to arise more generally, given that they originate from the exchange of KK states which are present in all compactifications.

The second type of correction comes from the exchange of winding strings between intersecting stacks of D7-branes (or between intersecting D7-branes and O7-planes). The exchanged strings are wound around non-contractible 1-cycles within the intersection locus of the D7-branes (and O7-planes, respectively), cf. fig. 6.2. This leads to the second kind of correction in 6.21 proportional to $\mathcal{E}_{i}^{W}$. The superscript $(W)$ reminds us that

\footnotetext{
${ }^{7}$ We should mention that there was no additional correction of this kind coming from KK exchange between (parallel) D7-branes in [171] (actually that paper considered the T-dual version with D5branes, but here we directly translate the result to the D7-brane language). This was due to the fact that in [171] the D7-brane scalars were set to zero. In general we would also expect a correction coming from parallel (or more generally, non-intersecting) D7-branes by exchange of KK-states. These should scale in the same way with the Kähler moduli as those arising from the KK exchange between D3- and D7-branes.
} 


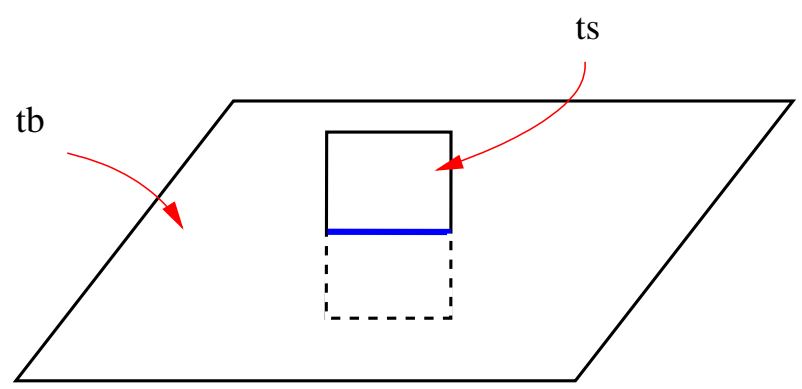

Figure 6.2: The loop correction $\mathcal{E}^{W}$ comes from the exchange of winding strings on the intersection between the small 4-cycle $\tau_{\mathrm{s}}$ and the large 4-cycle $\tau_{b}$. If this intersection is empty, there are no terms with $\mathcal{E}^{W}$.

these terms arise from the exchange of winding strings. In toroidal orientifolds, this type of correction is suppressed by the two Kähler moduli measuring the volumes of the 4cycles wrapped by the D7-branes (and O7-planes). One might a priori think that this kind of correction does not generalize easily to a smooth Calabi-Yau which has vanishing first Betti number (and therefore at most torsional 1-cycles). However, the exchanged winding strings are, from the open string point of view, Dirichlet strings with their endpoints stuck on the D7-branes. Thus, the topological condition is on the cycle over which the two D7-brane stacks (or one D7-brane stack and an O7-plane) intersect, as in figure 6.3. Thus, it depends on the topology of specific cycles within cycles whether winding open strings exist in a given model ${ }^{8}$

Given the expressions in [171] and the subset reproduced in (6.21) above, it is tempting to conjecture that some terms at one loop might be suppressed only by powers of single Kähler moduli like the $\tau_{i}$ (and the dilaton):

$$
\text { Calabi-Yau: } \quad \Delta K_{g_{\mathrm{s}}} \stackrel{?}{=} \frac{\mathcal{E}}{S_{1} \tau_{i}}
$$

for some function $\mathcal{E}$ of the complex structure and open string moduli. If this were the case, the one-loop corrections would typically dominate the $\alpha^{\prime}$ correction in (6.21) (which is suppressed by the overall volume $\mathcal{V}$ ) in the Kähler potential, if there are large hierarchies among the Kähler moduli. However, one should keep in mind that toroidal orientifolds are rather special in that they have very simple intersection numbers. In particular, the overall volume can be written as $\mathcal{V} \sim \tau_{i} t_{i}$, where there is no summation over $i$ implied. Thus, it is not obvious whether a generalization to the case of a general Calabi-Yau really contains terms suppressed by single Kähler moduli instead of the overall volume. Even though we can not exclude the presence of such terms, we deem it more likely that the

\footnotetext{
${ }^{8}$ The toroidal orientifold case seems to be a bit degenerate. Two stacks of D7-branes intersect along a 2-cycle with the topology of $\mathbb{P}^{1}$. However, there are point-like curvature singularities along the $\mathbb{P}^{1}$ at the orbifold point and strings winding around these singular points can not be contracted without crossing the singularities. This seems to allow for stability of winding strings (at least classically).
} 


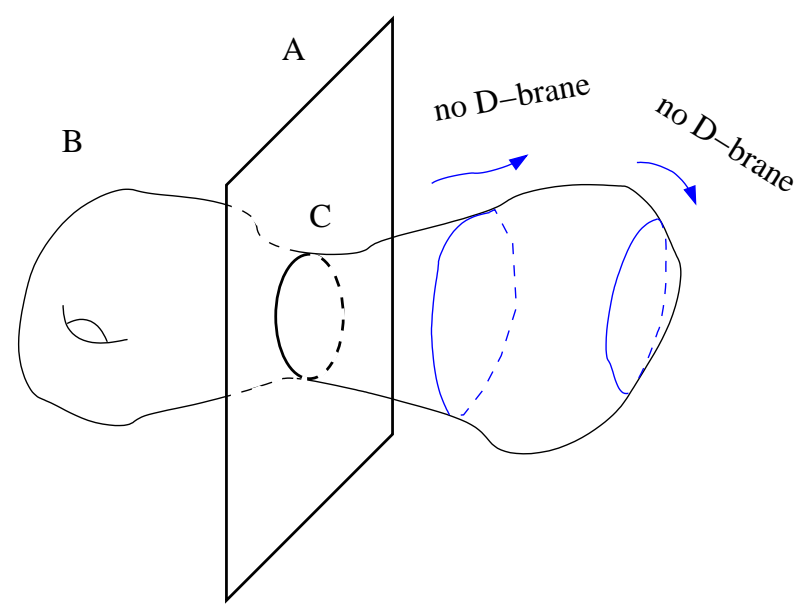

Figure 6.3: A D7-brane is wrapped on a 4-cycle A, which intersects the 4-cycle $\mathrm{B}$ on a 2-cycle C. For Dirichlet strings, the relevant topological condition (the existence of nontrivial 1-cycles) is on the intersection locus $\mathrm{C}$, not on cycle $\mathrm{B}$ or on the whole Calabi-Yau. In other words, without the D-brane, the string on cycle $\mathrm{C}$ could have been unwound by sliding it along cycle $\mathrm{B}$ (as shown in the figure). With the D-brane, the string on cycle $\mathrm{C}$ is stuck.

scaling of one-loop corrections to the Kähler potential is not 6.22 but

$$
\begin{aligned}
& \text { Calabi-Yau: } \quad \Delta K_{g_{\mathrm{s}}} \stackrel{!}{\sim} \frac{\sum_{\mathrm{KK}} m_{\mathrm{KK}}^{-2}}{S_{1} \mathcal{V}} \sim \sum_{\mathfrak{a}} \frac{g_{\mathrm{K}}^{\mathfrak{a}}\left(t, S_{1}\right) \mathcal{E}_{\mathfrak{a}}^{K}}{S_{1} \mathcal{V}} \\
& \text { and } \quad \frac{\sum_{\mathrm{W}} m_{\mathrm{W}}^{-2}}{\mathcal{V}} \sim \sum_{\mathfrak{q}} \frac{g_{\mathrm{W}}^{\mathfrak{q}}\left(t, S_{1}\right) \mathcal{E}_{\mathfrak{q}}^{W}}{\mathcal{V}} \text {, }
\end{aligned}
$$

where the sums run over KK and winding states, respectively. Also, $\mathcal{E}^{K}$ and $\mathcal{E}^{W}$ are again unknown functions of the complex structure and open string moduli, $t$ stands for the 2-cycle volumes (in the Einstein frame; see appendix D.3.1) and the functions $g_{\mathrm{K}}\left(t, S_{1}\right)$ and $g_{\mathrm{W}}\left(t, S_{1}\right)$ determine the scaling of the KK and winding mode masses with the Kähler moduli and the dilaton 9 As we review in appendix D.5, in the toroidal orientifold case the suppression by the overall volume arises naturally through the Weyl rescaling to the 4-dimensional Einstein frame.

Starting with the ansatz (6.23) for smooth Calabi-Yau manifolds, the known form 6.22 for toroidal orientifolds follows simply by substituting $g_{\mathrm{K}}, g_{\mathrm{W}}$ and the intersection numbers for the toroidal orientifold case. In particular, $g_{\mathrm{K}} \sim t_{i}$ for the 2-cycle transverse to the relevant D7-brane, while $g_{\mathrm{W}} \sim t_{i}^{-1}$ for the 2-cycle along which the two D7-branes intersect.

\footnotetext{
${ }^{9}$ In rewriting the sums over KK and winding states in terms of the functions $g$ and $\mathcal{E}$, we assume that the dependence of the corresponding spectra on the complex structure and Kähler moduli factorizes. In the known examples of toroidal orientifolds (with or without world-volume fluxes), this is always the case, cf. [181. Moreover, in general there can appear several contributions (denoted by $\mathfrak{a}$ and $\mathfrak{q}$ ) depending on which tower of KK or winding states are exchanged in a given process. We will see explicit examples of this in the following.
} 
Then, the first of the terms in 6.23 reduces to $\mathcal{E}_{i}^{K} /\left(S_{1} \tau_{i}\right)$ for toroidal orientifolds, the second to $\mathcal{E}_{i}^{W} /\left(\tau_{j} \tau_{k}\right)$ with $j \neq i \neq k$, cf 6.50$)$. Our strategy in the following chapters will therefore be to assume a scaling like (6.23) for the 1-loop corrections to the Kähler potential for general Calabi-Yau spaces.

As already mentioned, the dependence on the complex structure and open string moduli can not be inferred by analogy to the orientifold case. We parameterize our ignorance by keeping the expressions $\mathcal{E}$ in 6.23 as unknown functions of the corresponding moduli. Then we investigate the consequences of the one-loop terms, depending on the size of these unknown functions at the minimum of the potential for the complex structure and open string moduli. Some further comments on the form of $\Delta K_{g_{\mathrm{s}}}$ will appear in section 6.5 .2 .

\subsubsection{LVS with loop corrections}

Thus, allowing for string loop corrections of the form (6.23) in (6.9), and expanding the $\alpha^{\prime}$ correction as in $(6.10)$, we can write

$$
\begin{aligned}
K & =-\ln \left(2 S_{1}\right)-2 \ln (\mathcal{V})+K_{\mathrm{cs}}(u, \bar{u})-\frac{\tilde{\xi} S_{1}^{3 / 2}}{\mathcal{V}}+\sum_{\mathfrak{a}} \frac{g_{\mathrm{K}}^{\mathfrak{a}} \mathcal{E}_{\mathfrak{a}}^{K}}{S_{1} \mathcal{V}}+\sum_{\mathfrak{q}} \frac{g_{\mathrm{W}}^{\mathfrak{q}} \mathcal{E}_{\mathfrak{q}}^{W}}{\mathcal{V}} \\
W & =W_{0}+\sum_{i} A_{i} e^{-a_{i} T_{i}}
\end{aligned}
$$

where as explained in the previous section, we have not specified the explicit form of the loop corrections $\mathcal{E}$, that are allowed to be functions of $u$ (and in general of the open string moduli, that we neglect in our analysis, assuming that they can be stabilized by fluxes). The Kähler potential for the complex structure moduli $K_{\mathrm{cs}}(u, \bar{u})$ is left unspecified in (6.24), indeed we will not need its explicit form. For consistency, we have also included loop corrections to the $\alpha^{\prime}$ correction 10 This changes $\xi$ to $\tilde{\xi}$, which is a small change; for $S_{1}=10$, numerically $\tilde{\xi} \approx 1.02 \xi$.

Neglecting fluxes, the functions $g_{\mathrm{K}}^{\mathfrak{a}}$ and $g_{\mathrm{W}}^{\mathfrak{q}}$ are proportional and inversely proportional to some 2-cycle volume, respectively. (We will come back to corrections from fluxes in appendix D.4.) When using a particular basis of 2-cycles (with volumes $t_{i}$ as in appendix D.3.1), the 2-cycle volume appearing in $g_{\mathrm{K}}^{\mathfrak{a}}$ or $g_{\mathrm{W}}^{\mathfrak{q}}$ might be given by a linear combination $t_{\mathfrak{a}}=\sum_{i} c_{i} t_{i}$ of the basis cycles $t_{i}$ (and similarly for $t_{\mathfrak{q}}$ ). Depending on which 2-cycle is the relevant one, this linear combination might or might not contain the large 2-cycle $t_{b} \sim \mathcal{V}^{1 / 3}$, which always exists in LVS. If it is present in the linear combination, one can neglect the contribution of the small 2-cycles to leading order in a large volume expansion

\footnotetext{
${ }^{10}$ We remind the reader that the $\alpha^{\prime}$ correction arises from the $R^{4}$ term in 10 dimensions whose coefficient receives corrections at 1-loop (and from D-instantons). The 1-loop correction amounts to a shift of the prefactor from $\xi$ to $\tilde{\xi}=\xi\left(1+\frac{\pi^{2}}{3 \zeta(3) S_{1}^{2}}\right)$, see for instance [182 for a review.
} 
and obtains possible terms proportional to $\mathcal{E}_{b}^{K} S_{1}^{-1} \mathcal{V}^{-2 / 3}$ or $\mathcal{E}_{b}^{W} \mathcal{V}^{-4 / 3}$, where the subscript $b$ refers to the large 4 -cycle $\tau_{b}$.

Before getting into the details, it is hard to resist trying to anticipate what might happen. For those terms that are more suppressed in volume than the $\tilde{\xi}$ term (e.g $\mathcal{E}_{b}^{W}$ ), one would expect the loop corrections to have little effect on stabilization. They could still represent a small but interesting correction to physical quantities in LVS. For those that are less suppressed in volume than the $\tilde{\xi}$ term $\left(\right.$ e.g. $\left.\mathcal{E}_{b}^{K}\right)$, one would expect the loop correction to have a huge effect on stabilization, and severely constrain the allowed values for the complex structure moduli and the dilaton in LVS (in particular, constrain them to a region in moduli space where the function $\mathcal{E}_{b}^{K}$ takes very small values). We will find, however, that this expectation is sometimes too naive. For example, there can be cancellations in the scalar potential that are not obvious from just looking at the Kähler potential.

Let us now get into more detail on what happens in the LVS model with loop corrections.

\subsubsection{The $\mathbb{P}_{[1,1,1,6,9]}^{4}$ model}

We would now like to specify the general form of the Kähler- and superpotential (6.24) to the case of the $\mathbb{P}_{[1,1,1,6,9]}^{4}$ model. In this space, the divisors that produce nonperturbative superpotentials when D7- (or D3-) branes are wrapped around them do not intersect, as reviewed for instance in [178]. Therefore, we do not expect any correction of the $\mathcal{E}^{W}$ type in this model (for the generalization to models where there are such intersections, see appendix D.4). Moreover, we neglect flux corrections to the KK mass spectrum in the main text. It is shown in appendix D.4 that, for small fluxes, this correctly captures all the qualitative features we are interested in, and it leads to much clearer formulas. Thus, we now consider the scalar potential resulting from

$$
\begin{aligned}
K & =-\ln \left(2 S_{1}\right)-2 \ln (\mathcal{V})+K_{\mathrm{cs}}(u, \bar{u})-\frac{\tilde{\xi} S_{1}^{3 / 2}}{\mathcal{V}}+\frac{\sqrt{\tau_{b}} \mathcal{E}_{b}^{K}}{S_{1} \mathcal{V}}+\frac{\sqrt{\tau_{s}} \mathcal{E}_{s}^{K}}{S_{1} \mathcal{V}} \\
W & =W_{0}+A e^{-a T_{s}}
\end{aligned}
$$

As $\tau_{b}$ is very large the corresponding non-perturbative term in the superpotential of (6.24) can be neglected, which allowed us to simplify the notation by setting $A_{s}=A$ and $a_{s}=a$.

The general structure of the scalar potential was already given in 6.12). The three 
contributions at leading order $\left(\mathcal{O}\left(\mathcal{V}^{-3}\right)\right)$ in the large volume expansion are

$$
\begin{aligned}
V_{\mathrm{np} 1} & =e^{K_{\mathrm{cs}}} \frac{24 a^{2}|A|^{2} \tau_{s}^{3 / 2} e^{-2 a \tau_{s}}}{\mathcal{V} \Delta}, \\
V_{\mathrm{np} 2} & =-e^{K_{\mathrm{cs}}} \frac{2 a\left|A W_{0}\right| \tau_{s} e^{-a \tau_{s}}}{S_{1} \mathcal{V}^{2}}\left[1+\frac{6 \mathcal{E}_{s}^{K}}{\Delta}\right], \\
V_{3} & =\frac{3 e^{K_{\mathrm{cs}}}\left|W_{0}\right|^{2}}{8 \mathcal{V}^{3}}\left[S_{1}^{1 / 2} \tilde{\xi}+\frac{4\left(\mathcal{E}_{s}^{K}\right)^{2} \sqrt{\tau_{s}}}{S_{1}^{2} \Delta}\right],
\end{aligned}
$$

where the axion has already been minimized for, as discussed in section D.1.2, and

$$
\Delta \equiv \sqrt{2} S_{1} \tau_{s}-3 \mathcal{E}_{s}^{K}
$$

The leading $\alpha^{\prime}$-correction is the $\tilde{\xi}$ term in $V_{3}$ above. We now see that it scales with the volume and the string coupling $g_{\mathrm{s}}=1 / S_{1}$ as claimed in the Introduction, in (6.3). Also the volume dependence of the loop correction $\left(\mathcal{E}_{s}^{K}\right.$ term) in $V_{3}$ is as announced in 6.3). The $g_{\mathrm{s}}$ factors seem to differ from (6.3); we see $g_{s}^{2}, g_{s}^{2}$ and $g_{s}^{3}$ for $V_{\mathrm{np} 1}, V_{\mathrm{np} 2}$ and $V_{3}$, respectively. This is because the $g_{\mathrm{s}}$ dependence advertised in (6.3) arises in models where, unlike in $\mathbb{P}_{[1,1,1,6,9]}^{4}$, the $\mathcal{E}^{W}$ correction is present as well, cf. appendix D.4 It is also worth mentioning that the loop correction proportional to $\mathcal{E}_{s}^{K}$ modifies $V_{\mathrm{np} 1}$ and $V_{\mathrm{np} 2}$ at leading order in the $\mathcal{V}$-expansion. whereas the $\alpha^{\prime}$ correction does not; it only appears in $V_{3}$. This is so even though both corrections are equally suppressed in the Kähler potential (i.e. $\sim \mathcal{V}^{-1}$ ). The reason for this can be traced back to the fact that the loop-correction explicitly depends on $\tau_{s}$ and not only on the overall volume, cf. the discussion in appendix D.3.4 and D.3.5.

As anticipated, $\mathcal{E}_{b}^{K}$ and its first derivatives appear only at the next order, $\mathcal{O}\left(\mathcal{V}^{-10 / 3}\right)$ :

$$
V_{10 / 3}=2 \frac{6^{1 / 3}\left|W_{0}\right|^{2} e^{K_{\mathrm{cs}}}}{S_{1}^{3} \mathcal{V}^{10 / 3}}\left[\left(\mathcal{E}_{b}^{K}\right)^{2}+\frac{3}{4} \partial_{\alpha} \mathcal{E}_{b}^{K} \partial_{\bar{\alpha}} \mathcal{E}_{b}^{K} K_{\mathrm{cs}}^{\alpha \bar{\alpha}}\right],
$$

where $\partial_{\alpha}=\partial / \partial u^{\alpha}$ and $\partial_{\bar{\alpha}}=\partial / \partial \bar{u}^{\bar{\alpha}}$ and $\alpha$ enumerates the complex structure moduli. For $\mathcal{E}_{b}^{K}=\mathcal{E}_{s}^{K}=0$, the potential terms at leading order coincide with the original case discussed in (D.1), cf. appendix D.1.1. The singularity from zeros of the denominator is an artifact of the expansion as discussed in appendix D.2. The range of validity is limited to the range in moduli space where the denominator $\Delta$ does not become too small. It is also apparent that the loop terms are subleading in a large $\tau_{s}$, large $S_{1}$ expansion. However, depending on the relative values of the parameters $\left\{\mathcal{E}_{s}^{K}, \tau_{s}, S_{1}\right\}$, a truncation to the first terms in such an expansion may or may not be valid. We perform a numerical comparison of the two contributions to $V_{3}$ in figure 6.4.

\footnotetext{
${ }^{11}$ There, it is shown that including the effect of fluxes on the KK spectrum might also produce this behavior.
} 


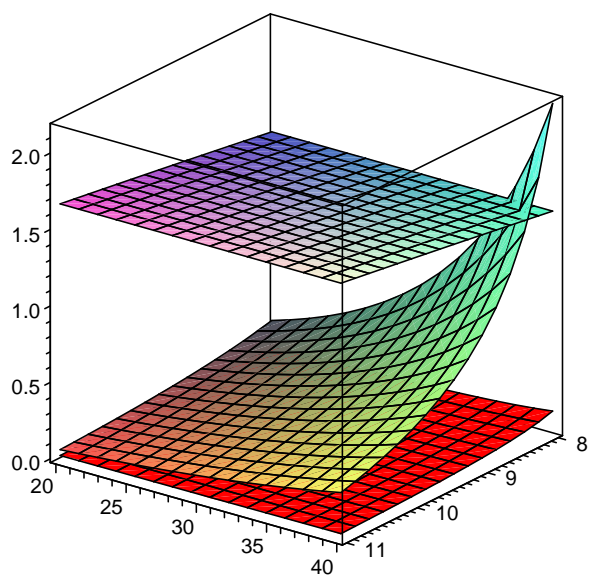

Figure 6.4: The top surface is the $\alpha^{\prime}$ correction, the second is the $g_{\mathrm{s}}$ correction, and the "red carpet" is $10 / \Delta$ (we used the values $A=1, W_{0}=1, a=2 \pi / 8$ ). We see that for most of the parameter range, the $\alpha^{\prime}$ correction dominates, and only for large $\mathcal{E}_{s}^{K}$, with the string coupling $g_{\mathrm{s}}=1 / S_{1}$ not too small, do the contributions become comparable.

We can understand the volume dependence of the terms $(6.26)-(\sqrt{6.30})$ as follows. The common prefactor $e^{K}$ gives an overall suppression $\tau_{b}^{-3} \simeq \mathcal{V}^{-2}$. The quantum corrections obey the rule that a term proportional to $1 / \tau_{b}^{\lambda}$ in $K$ appears in $V_{3}$ at order $1 / \tau_{b}^{\lambda+3}$ (where the +3 comes from the overall $e^{K}$ factor) for all values of $\lambda$ except for $\lambda=1$. When it does appear, it is generated by the term $\left(K^{i} K_{i}-3\right)$ and breaks the no-scale structure. For $\lambda=1$ there is a cancellation at leading order, so it appears only at order $1 / \tau_{b}^{2+3}$ (see appendix D.3.3 and D.3.4 . This rule can explicitly be verified in our calculation: the $\alpha^{\prime}$ and the $\mathcal{E}_{s}^{K}$ corrections are suppressed by $1 / \tau_{b}^{3 / 2}$ in $K$, and therefore they appear with the suppression $1 / \tau_{b}^{9 / 2}$ in $V_{3}$. On the other hand, for the $\mathcal{E}_{b}^{K}$ term a cancellation takes place to leading order $(\lambda=1)$. It appears neither in $V_{\mathrm{np} 1}$ nor in $V_{\mathrm{np} 2}$ at leading order (which can be understood more generally, cf. appendix D.3.5). Thus, it only appears subleading in the potential, at $\mathcal{O}\left(\mathcal{V}^{-10 / 3}\right) .12$

We now proceed to minimize the potential (6.26)-(6.28), using the same strategy as in the case without loop corrections, cf. section 6.2.3 and appendix D.1.1. The equations $\partial_{\mathcal{V}} V=0=\partial_{\tau_{s}} V$ are of course more complicated now, but it is easy to solve them numerically. Doing so we find that the volume $\mathcal{V}$ and the small 4-cycle volume $\tau_{s}$, viewed

\footnotetext{
${ }^{12}$ This cancellation for $\lambda=1$ was already noticed in [91, albeit in the case without nonperturbative superpotential. In [183] it was argued that this cancellation can be understood from a field redefinition argument combined with the no-scale structure of the tree-level Kähler potential. That argument holds for the case of a single Kähler modulus $T$ with tree-level Kähler potential $-3 \ln (T+\bar{T})$ and under the assumption that the coefficient of the loop correction to the Kähler potential $\sim(T+\bar{T})^{-1}$ is independent of the complex structure moduli and the dilaton. Here, these assumptions do not hold, but we showed that the term $\sim\left(T_{b}+\bar{T}_{b}\right)^{-1}$ in the Kähler potential nevertheless only appears at subleading order in the potential in LVS, cf. (6.26)- (6.30).
} 
as functions of $S_{1}$ and $\mathcal{E}_{s}^{K}$, are well fit by linear functions when restricted to a sufficiently limited range in parameter space. For example,

$$
\begin{aligned}
\text { range: } S_{1}=[8,11], \mathcal{E}_{s}^{K} & =[20,40] \\
\log _{10} \mathcal{V} & =1.720 S_{1}-0.1208 \mathcal{E}_{s}^{K}-3.437, \\
\tau_{s} & =5.000 S_{1}-0.3581 \mathcal{E}_{s}^{K}-8.638 .
\end{aligned}
$$

The fits are quite good; the error is no greater than \pm 0.3 for $\tau_{s}$ and \pm 0.1 for $\log _{10} \mathcal{V}$ in this range, for an $\left\{S_{1}, \mathcal{E}_{s}^{K}\right\}$ grid of $40^{2}$ points.

From (6.31) we see an interesting difference to the case without loop corrections. The value of $\tau_{s}$ at the minimum depends on the complex structure moduli $u$, through $\mathcal{E}_{s}^{K}$. This is in contrast to the case without loop corrections, where the value of $\tau_{s}$ is only determined by the value of the Euler number $\xi$ and the dilaton $S_{1}$, cf. (D.2) below. It is analogous to the perturbative stabilization in [91] where the volume at the minimum of the potential also depends on $u$.

The result (6.26)- 6.28$)$ was derived in a particular model, but we expect the appearance of loop corrections in $V$ to be more general. This opens up the possibility that in principle, one might obtain large volume minima even for manifolds of vanishing (or even positive) Euler number, where LVS is not applicable, as LVS-style stabilization only holds for one sign of $\xi$. In practice it might be difficult to get large enough values for the 1-loop corrections to stabilize $\tau_{s}$ at a value sufficiently bigger than the string scale. This deserves further study.

We also note that the special structure of 6.28) and 6.30, i.e. the appearance of $\mathcal{E}_{s}$ only in 6.28) and of $\mathcal{E}_{b}$ only in 6.30), offers additional flexibility in tuning the relative size of these terms in a purely perturbative stabilization of the Kähler moduli along the lines of [90, 91]. Also this point deserves further study.

\subsection{Gaugino masses}

Now that we know how the stabilization of the (Kähler) moduli is modified by loop corrections, it is natural to extend our analysis to the soft supersymmetry breaking Lagrangian (For a review see for instance [184, 185].) In LVS, supersymmetry breaking is mostly due to $F$-terms: $F_{s} \neq 0, F_{b} \neq 0$. These determine the soft supersymmetry breaking terms which can be present in the low energy effective action without spoiling the hierarchy between the electroweak and the Planck scale,

$$
\mathcal{L}_{\text {eff }}=\mathcal{L}_{\mathrm{MSSM}}+\mathcal{L}_{\text {soft }}
$$

The soft Lagrangian contains gaugino masses $M$, scalar masses $m$, further scalar bilinear terms $B$ and trilinear terms $A$. (For explicit expressions, see the aforementioned reviews, or e.g. [144].) 
Let us start considering gaugino masses. In [144] it was shown that in LVS, gaugino masses $M_{a}$ are generically suppressed with respect to the gravitino mass $m_{3 / 2}$ :

$$
\left|M_{a}\right| \simeq \frac{m_{3 / 2}}{\ln \left(1 / m_{3 / 2}\right)}\left[1+\mathcal{O}\left(\frac{1}{\ln \left(1 / m_{3 / 2}\right)}\right)\right]
$$

(we use units in which $M_{\mathrm{Pl}}=1$ ). This suppression results from a cancellation of the leading order $F$-term contribution to gaugino masses. We briefly review this calculation. Given the $F$-terms

$$
F^{I}=e^{K / 2} G^{\bar{J} I} D_{\bar{J}} \bar{W},
$$

gaugino masses are given by 184

$$
M_{a}=\frac{1}{2} \frac{1}{\operatorname{Re} f_{a}} \sum_{I} F^{I} \partial_{I} f_{a},
$$

where $f_{a}$ are the gauge kinetic functions and $a$ labels the different gauge group factors. In LVS the Standard Model (SM) gauge groups arise from D7-branes wrapped around small 4-cycles. We do not try to go into the details of how to embed the SM concretely, but we mention that different gauge group factors might arise from brane stacks wrapping the same 4-cycle if world volume fluxes are present on the branes. In that case the gauge kinetic functions are given by ${ }^{13}$

$$
f_{a}=\frac{T_{a}}{4 \pi}+h_{a}(\mathcal{F}) S+f_{a}^{(1)}(u)
$$

where $h_{a}$ depends on the world volume fluxes and we also included a possible 1-loop correction to the gauge kinetic function which depends on the complex structure (and possibly open string) moduli. If several gauge groups arise from branes wrapped around the same cycle, the same Kähler modulus $T$ would appear in all of them. From (6.36) it is clear why the D7-branes of the SM have to wrap small 4-cycles, because otherwise the gauge coupling would come out too small (unless there is an unnatural cancellation between the different contributions to $f_{a}$ ).

As is also apparent from (6.36), the gauge kinetic function in general depends not only on the Kähler moduli but also on the dilaton and the complex structure. Thus, according to 6.35) we need to know $F^{u}, F^{S}$ and $F^{i}$ for the small Kähler moduli. ${ }^{14}$ From the definition (6.34), it is clear that $F^{u}$ and $F^{S}$ might be non-vanishing even though we assume $D_{u} W=0=D_{S} W$, provided the inverse metric $G^{\bar{J} I}$ contains mixed components between Kähler moduli on the one hand and complex structure moduli and dilaton on

\footnotetext{
${ }^{13}$ We use the "phenomenology" normalization of the gauge generators, in the language of [186]; that explains the relative factor of $4 \pi$ in 6.36 .

${ }^{14}$ With a slight abuse of notation, we denote the $F$-terms of the Kähler moduli by the index $i$, but the $F$-terms of the other moduli are identified by the symbol for the corresponding modulus, like $F^{S}$. This is to avoid introducing too many indices.
} 
the other hand. Without loop corrections (i.e. considering only the leading $\alpha^{\prime}$ correction) there is no mixing between the Kähler and complex structure moduli, and one finds

$$
F^{u}=0, \quad F^{S} \sim \mathcal{O}\left(\mathcal{V}^{-2}\right) \quad \text { and } \quad F^{i} \sim \mathcal{O}\left(\mathcal{V}^{-1}\right) \quad \text { (without loop corrections) }
$$

Thus, at leading order in the large volume expansion, the sum in 6.35 only runs over the Kähler moduli. Moreover, taking into account the linear dependence of the gauge kinetic functions 6.36) on the (small) Kähler moduli, the sum effectively only involves a single term, i.e.

$$
M_{a}=\frac{1}{8 \pi} \frac{1}{\operatorname{Re} f_{a}} F^{a}+\mathcal{O}\left(\mathcal{V}^{-2}\right)
$$

where $F^{a}$ is the F-term of the (small) Kähler modulus appearing in $f_{a}$.

As a concrete example we consider again the $\mathbb{P}_{[1,1,1,6,9]}^{4}$ model with only one small Kähler modulus $\tau_{s}$. The corresponding F-term is given by

$$
\begin{aligned}
F^{s} & =e^{K / 2}\left(G^{\bar{s} s} \partial_{\bar{s}} \bar{W}+\left(G^{\bar{s} s} K_{\bar{s}}+G^{\bar{b} s} K_{\bar{b}}\right) \bar{W}\right) \\
& =2 \tau_{s} e^{K / 2} \bar{W}_{0}\left(\left(1-\frac{3}{4 a \tau_{s}}\right)-1+\mathcal{O}\left(\left(a \tau_{s}\right)^{-2}\right)\right)+\mathcal{O}\left(\mathcal{V}^{-2}\right)
\end{aligned}
$$

where we used (6.20) and (D.6) for the first term and (D.9) for the second.

Now the leading order cancellation is obvious in (6.39). Determining the gaugino masses requires dividing by $\operatorname{Re} f_{s}$, cf. (6.35). In order to further evaluate this, [144, 145] assumed that the dilute flux approximation $f_{s}=(4 \pi)^{-1} T_{s}$ is valid, i.e. they neglected the contributions from world-volume fluxes and one-loop terms compared to the tree-level term. This puts some constraints on the allowed discrete flux values determining $h_{s}$. We want to stress that the cancellation appearing in (6.39) is independent of this approximation. We are mainly interested in the fate of this cancellation when including loop corrections, and do not have anything to add concerning phenomenological constraints that may arise from imposing the dilute flux approximation. Using it, the gaugino masses simplify to

$$
\begin{aligned}
\left|M_{s}\right| & =\left|\frac{F^{s}}{2 \tau_{s}}\right|=e^{K / 2}\left|W_{0}\right|\left(\frac{3}{4 a \tau_{s}}+\mathcal{O}\left(\left(a \tau_{s}\right)^{-2}\right)\right) \\
& \sim \frac{m_{3 / 2}}{\ln \left(1 / m_{3 / 2}\right)}\left[1+\mathcal{O}\left(\frac{1}{\ln \left(1 / m_{3 / 2}\right)}\right)\right],
\end{aligned}
$$

which is the announced result. In 6.40 we used

$$
m_{3 / 2} \sim\left|W_{0}\right| / \mathcal{V} \quad \text { and } \quad a \tau_{s} \sim \ln \left(\mathcal{V} /\left|W_{0}\right|\right)
$$

where the second relation holds in LVS due to (6.20). 


\subsubsection{Including loop corrections}

The previous section was a review of the results found in [144]. Now we ask what changes if one considers the loop corrected Kähler potential (6.25). A priori, as (6.40) results from a leading order cancellation, one might wonder whether loop corrections might spoil this small hierarchy between the gaugino and gravitino masses. To address this concern we start by observing that the gaugino masses are still determined by the F-terms of the small Kähler moduli (in the large volume limit). More precisely, the scaling of the F-terms 6.37) now becomes

$$
F^{u}=\mathcal{O}\left(\mathcal{V}^{-2}\right), \quad F^{S} \sim \mathcal{O}\left(\mathcal{V}^{-2}\right) \quad \text { and } \quad F^{i} \sim \mathcal{O}\left(\mathcal{V}^{-1}\right) \quad \text { (with loop corrections) }
$$

i.e. $F^{u}$ no longer vanishes, but it is just as suppressed as $F^{S}$.

We again focus on the $\mathbb{P}_{[1,1,1,6,9]}^{4}$ model and ask how 6.39 is modified by loop corrections. Amongst other things, we need to generalize equation $(6.20)$ to include loop corrections, since we need it to calculate the first term in (6.39). Thus, we need to extremize the potential again with respect to $\tau_{s}$ by setting

$$
\begin{aligned}
\partial_{\tau_{s}} V= & \left\{-\frac{12 \sqrt{\tau_{s}} a^{2}}{\mathcal{V} \Delta^{2}}\left[\left(4 a \tau_{s}-1\right) \Delta+6 \mathcal{E}_{s}^{K}\right] X^{2}\right. \\
& +\frac{2 a\left|W_{0}\right|}{\mathcal{V}^{2} S_{1} \Delta^{2}}\left[\left(a \tau_{s}-1\right)\left(\Delta^{2}-18\left(\mathcal{E}_{s}^{K}\right)^{2}\right)+6 \sqrt{2} a S_{1} \tau_{s}^{2} \mathcal{E}_{s}^{K}\right] X \\
& \left.-\frac{3\left|W_{0}\right|^{2}\left(\mathcal{E}_{s}^{K}\right)^{2}}{4 S_{1}^{2} \mathcal{V}^{3} \Delta \sqrt{\tau_{s}}}\right\} e^{K_{\mathrm{cs}}}
\end{aligned}
$$

to zero. Obviously, $X=0$ is no longer a solution. Instead, there are now two non-trivial solutions, one of which goes to zero in the limit $\mathcal{E}_{s}^{K} \rightarrow 0$. This solution corresponds to a maximum of the potential, so it is of no use to us here. We can expand the other solution for large $a \tau_{\mathrm{s}}$, as in the case without loop corrections, yielding

$$
X=A e^{-a \tau_{s}}=\frac{\sqrt{2}\left|W_{0}\right|}{24 a \mathcal{V}} \sqrt{\tau_{s}}\left(1-\frac{3}{4 a \tau_{s}}\left(1-\frac{2 \sqrt{2} a \mathcal{E}_{s}^{K}}{S_{1}}\right)\right)+\mathcal{O}\left(\frac{1}{\left(a \tau_{s}\right)^{2}}\right) .
$$

Another ingredient we need is the quantity $G^{\bar{s} s} K_{\bar{\imath}}$, in order to evaluate the second term in (6.39). Using equation (D.10) we obtain

$$
\begin{aligned}
G^{\bar{\imath} s} K_{\bar{\imath}} & =-2 \tau_{s}\left(1+\frac{6 \mathcal{E}_{s}^{K}}{\Delta}\right)+\ldots \\
& =-2 \tau_{s}-\frac{6 \sqrt{2} \mathcal{E}_{s}^{K}}{S_{1}}-\frac{18\left(\mathcal{E}_{s}^{K}\right)^{2}}{S_{1}^{2} \tau_{s}}-\frac{27 \sqrt{2}\left(\mathcal{E}_{s}^{K}\right)^{3}}{\tau_{s}^{2} S_{1}^{3}}+\mathcal{O}\left(\frac{1}{\tau_{s}^{3}}\right)+\ldots
\end{aligned}
$$

where the ellipsis represents terms that are more suppressed in $\mathcal{V}^{-1}$. 
Now we see from (6.44), 6.45) and (D.10) that at leading order in an expansion in $a \tau_{s}$, the quantities relevant to evaluate (6.39) are not affected by the loop corrections. Thus, the leading order cancellation in the gaugino masses survives the inclusion of loop effects 15 At first glance, though, equations (6.44), 6.45) and (D.10) seem to suggest a correction to the subleading term, that could potentially give a significant contribution to the gaugino masses after the leading-order cancellation, cf. (6.39).

In the actual calculation, this contribution drops out. Putting all the ingredients together (and employing the dilute flux approximation again), the gaugino mass turns out to be

$$
\begin{aligned}
\left|M_{s}\right| & =\left|\frac{F^{s}}{2 \tau_{s}}\right|=3 e^{K / 2}\left|W_{0}\right|\left|\frac{1}{4 a \tau_{s}}+\frac{1}{16 a^{2} \tau_{s}^{2}}+\frac{S_{1}-12 \sqrt{2} a \mathcal{E}_{s}^{K}}{64 S_{1} a^{3} \tau_{s}^{3}}+\ldots\right| \\
& \sim \frac{m_{3 / 2}}{\ln \left(1 / m_{3 / 2}\right)}\left[1+\mathcal{O}\left(\frac{1}{\ln \left(1 / m_{3 / 2}\right)}\right)\right] .
\end{aligned}
$$

The result of [144] is therefore very robust. Unexpectedly, the correction to 6.40) due to $\mathcal{E}_{s}^{K}$ only appears at sub-sub-leading order in the $1 / \ln \left(1 / m_{3 / 2}\right)$ expansion.

\subsubsection{Other soft terms}

In [145] all other soft terms were calculated for LVS. The main result (see p. 15 of [145]) is that roughly speaking, all the soft parameters are determined by $F^{s}$ and by the power with which the chiral matter metrics scale with $\tau_{s}$. As we saw in the previous section, $F^{s}$ gets modified by loop corrections only at sub-sub-leading order in a $1 / \tau_{s}$ expansion (see (6.46)). Therefore, the calculation of all the soft terms in [145] appears to be quite robust against including loop effects.

One of the key assumptions in [145] is that all the Yukawa couplings $Y$ are already present in perturbation theory, i.e. they have the schematic form $Y=Y^{\text {pert }}(u)+Y^{\mathrm{np}}\left(e^{-T}\right)$. This requirement featured prominently already in the derivation of the volume dependence of the chiral matter metrics in [187] by scaling arguments. In [145] the same schematic form is essential for simplifying the trilinear soft terms $A$. In general these terms receive a contribution of the schematic form

$$
F^{T} \partial_{T} \log Y=F^{T} \frac{\partial_{T}\left(Y^{\text {pert }}(u)+Y^{\mathrm{np}}\left(e^{-T}\right)\right)}{Y^{\operatorname{pert}}(u)+Y^{\mathrm{np}}\left(e^{-T}\right)} \sim \frac{\mathcal{O}\left(e^{-T}\right)}{\mathcal{O}\left(T^{0}\right)+\mathcal{O}\left(e^{-T}\right)},
$$

which is exponentially suppressed if and only if $Y^{\text {pert }}(u)$ is non-vanishing. However, in many examples the Yukawa couplings are actually only generated nonperturbatively, see

\footnotetext{
${ }^{15}$ One might argue that this result was to be expected, because the main assumption of [144 is that the stabilization is due to nonperturbative effects, i.e. the dominant effect in $\partial_{\tau_{s}} V$ should arise from the nonperturbative superpotential. However, in view of 6.43 , it is no longer obvious that the nonperturbative terms dominate when loop corrections are included.
} 
for instance the discussion in [188], and [189] for some examples. This poses a constraint on the way the Standard Model is realized in LVS, if one wants to ensure flavor universality of the soft breaking terms as advertised in [145].

One more comment about the important issue of flavor universality. In [145], section 3.4., it was argued that in LVS, approximate flavor universality is a natural consequence of the zeroth-order factorization of Kähler and complex structure moduli spaces. We provide some more details on the factorized approximation in appendix D.6.

\subsection{LVS for other classes of Calabi-Yau manifolds?}

In section 6.3 .3 and 6.4 we saw that the 1-loop corrections to the moduli Kähler potential only have relatively small effects on the large volume scenario based on the $\mathbb{P}_{[1,1,1,6,9]}^{4}$ model of [169]. In this chapter, we would like to ask the question how generic the "Swiss cheese" form is for a Calabi-Yau manifold and if there are other models in which the one-loop corrections discussed above might become more important. This is indeed to be expected if the Calabi-Yau under consideration has a fibered structure, as we explain in the following. If $g_{\mathrm{s}}$ corrections do dominate $\alpha^{\prime}$ corrections, they could ruin the volume expansion of LVS.

\subsubsection{Abundance of "Swiss cheese" Calabi-Yau manifolds}

In the LVS examples discussed in [143] the volume in terms of the Kähler moduli takes the "Swiss cheese" form

$$
\mathcal{V}=\left(\tau_{b}+\sum a_{i} \tau_{i}\right)^{3 / 2}-\left(\sum b_{i} \tau_{i}\right)^{3 / 2}-\ldots-\left(\sum c_{i} \tau_{i}\right)^{3 / 2}
$$

where the coefficients $a_{i}, \ldots, c_{i}$ are only non-vanishing for the small Kähler moduli. The LVS limit consists in scaling the overall volume of the Calabi-Yau more or less isotropically while having small holes inside the manifold. The $\tau$ 's are linear combinations of $\partial_{t_{i}} \mathcal{V}$, where now $\mathcal{V}$ is considered as a (cubic) function of the 2 -cycle volumes $t_{i}$. For the effective field theory analysis to be valid one should not only demand that the 4-cycle volumes $\tau_{i}$ are large compared to the string scale, but also that the 2-cycle volumes $t_{i}$ are large. In the cases discussed in [143], the linear combinations $\partial_{t_{i}} \mathcal{V}$ are indeed such that one can have one of them exponentially large and the others small (but still sufficiently larger than the string scale), without taking any of the $t_{i}$ to be exponentially small. This is obvious for the $\mathbb{P}_{[1,1,1,6,9]}^{4}$ example where the 2-cycle volume $t_{b}$ only appears in the definition of one of the $\tau$ 's, cf. 6.14), but it is also true for the second example of [143], cf. their formulas (84). 
However, the $\mathcal{F}_{18}$ model of [61] does not seem to allow its volume to be written in the form 6.48 with one Kähler modulus $\tau_{b}$ that can become large while keeping all the others small (again, demanding that the $t_{i}$ stay larger than 1 in string units). Thus, it is an interesting question how generic or non-generic the "Swiss cheese" Calabi-Yau manifolds are. We do not attempt to give a general answer; instead, we turn to two examples in which the form of the volume differs from (6.48).

\subsubsection{Toroidal orientifolds}

The reason loop corrections may be more important in toroidal orientifolds than in compactifications on "Swiss cheese" Calabi-Yau manifolds is the following. As we already discussed in section 6.3.1, the conjectured form of 1-loop corrections (6.23) simplifies in the case of toroidal orientifolds, because they have very special and simple intersection numbers. More concretely, using the definition $\tau_{i}=\partial_{t_{i}} \mathcal{V}$, together with the special form of the intersection numbers in the toroidal case, i.e. $\mathcal{V}=t_{1} t_{2} t_{3}$, the volume can alternatively be expressed as

$$
\mathcal{V}=\sqrt{\tau_{1} \tau_{2} \tau_{3}}=t_{i} \tau_{i} \quad(\text { no summation; } i=1,2 \text { or } 3)
$$

Thus, formula 6.23 simplifies and the 1-loop corrections proportional to $\mathcal{E}_{i}^{K}$ are only suppressed by single Kähler moduli instead of by the overall volume. Also the terms proportional to $\mathcal{E}_{i}^{W}$ can be rewritten in the toroidal orientifold case and the Kähler potential takes the form (for the $T^{6} /\left(\mathbb{Z}_{2} \times \mathbb{Z}_{2}\right)$ example)

$$
\begin{aligned}
K=-\ln \left(2 S_{1}\right)- & 2 \ln (\mathcal{V})+K_{\mathrm{cs}}(u, \bar{u})-\frac{\xi S_{1}^{3 / 2}}{\mathcal{V}} \\
& +\sum_{i=1}^{3} \frac{\mathcal{E}_{i}^{K}(u, \bar{u})}{4 \tau_{i} S_{1}}+\sum_{i \neq j \neq k}^{3} \frac{\mathcal{E}_{k}^{W}(u, \bar{u})}{4 \tau_{i} \tau_{j}},
\end{aligned}
$$

where the functions $\mathcal{E}$ are non-holomorphic Eisenstein series in this case [171]. It is easy to see that the origin of this simplification is the fact that there is just a single non-vanishing intersection number in the toroidal orientifold case and all Kähler moduli appear linearly in the cubic expression for the volume.

The difference of the toroidal orientifold to the "Swiss cheese" case of LVS can also be seen in the different forms of the functional dependence of the volume on the Kähler moduli. In the toroidal orientifold case one has the relations

$$
\partial_{t_{1}} \mathcal{V}=t_{2} t_{3} \quad, \quad \partial_{t_{2}} \mathcal{V}=t_{1} t_{3} \quad, \quad \partial_{t_{3}} \mathcal{V}=t_{1} t_{2}
$$

so that two of them will always become large if one takes one of the $t_{i}$ to be large and demands that the other two stay larger than 1 . This also holds for any linear combinations 
of them. The difference is also obvious from the fact that the 2 -cycle volume $t_{b}$ that is responsible for $\tau_{b}$ becoming large in the LVS examples of [143] always appears cubically in the volume. This is related to the fact that the term $\left(\tau_{b}+\sum a_{i} \tau_{i}\right)$ should be the square of a linear combination of the $t_{i}$, in order for (6.48) to be expressible as a cubic polynomial in the $t_{i}$. In contrast, any (untwisted) 2-cycle volume in the toroidal orientifold case only appears linearly in the cubic volume polynomial.

To illustrate the effect of terms in the Kähler potential that are suppressed only by single Kähler moduli instead of the overall volume, we take the Kähler potential 6.50) and expand $V_{3}$ in the region of the Kähler cone where $\tau_{1}=\tau_{2}=\tau_{b} \gg \tau_{3}=\tau_{s}$ (as we explained above, at least two of the Kähler moduli have to become large simultaneously, if one wants to avoid any of the 2-cycle volumes becoming very small). This leads to (for simplicity setting all $u_{i}=u$, all $\mathcal{E}_{i}^{K}=\mathcal{E}^{K}$ and all $\left.\mathcal{E}_{i}^{W}=\mathcal{E}^{W}\right)$ :

$$
\begin{aligned}
V_{3}= & \frac{\left|W_{0}\right|^{2} e^{K_{\mathrm{cs}}}}{2 S_{1} \mathcal{V}^{2}}\left\{\left[\frac{\left(\mathcal{E}^{K}\right)^{2}+\frac{1}{2}\left(\partial_{u \bar{u}} K_{\mathrm{cs}}\right)^{-1} \partial_{u} \mathcal{E}^{K} \partial_{\bar{u}} \mathcal{E}^{K}}{8 \tau_{s}^{2} S_{1}^{2}}+\mathcal{O}\left(\frac{1}{\tau_{s}^{3}}\right)\right]\right. \\
& +\frac{1}{\tau_{b}}\left[\frac{3 \tilde{\xi} S_{1}^{3 / 2}}{4 \sqrt{\tau_{s}}}+\frac{\mathcal{E}^{W}}{\tau_{s}}+\frac{\left(\mathcal{E}^{K}\right)^{2}+\left(\partial_{u \bar{u}} K_{\mathrm{cs}}\right)^{-1} \partial_{u} \mathcal{E}^{K} \partial_{\bar{u}} \mathcal{E}^{K}}{4 S_{1}^{2} \tau_{s}}+\mathcal{O}\left(\frac{1}{\tau_{s}^{3 / 2}}\right)\right] \\
& \left.+\mathcal{O}\left(\frac{1}{\tau_{b}^{2}}\right)\right\} .
\end{aligned}
$$

Obviously, the leading term in the large $\tau_{b}$ expansion now comes from the loop correction and not from the $\alpha^{\prime}$ term (which term really dominates depends on the values of $S_{1}$ and $u$ as well, of course). Thus, an expansion of the potential as in LVS, cf. (6.16), would not be realized in this case, even if one found a way to lift enough zero modes by fluxes for $\tau_{s}$ to appear in a nonperturbative superpotential.

This toy example was meant to show that for a consistent large volume expansion in models with large hierarchies in the Kähler moduli, it is important to make sure that there are no correction terms in the Kähler potential (from loop or $\alpha^{\prime}$-corrections) that are suppressed only by some of the small Kähler moduli. We should stress again that also terms suppressed by the large volume can be dangerous if the suppression is less than for the $\alpha^{\prime}$ term, i.e. if it is $\tau_{b}^{-\lambda}$ with $\lambda<3 / 2$. The only exception to this rule is the case $\lambda=1$ as we showed above (and as is shown more generally in appendices D.3.4 and D.3.5). In this respect it would be important to know if the conjecture 6.25 really bears out. If it turns out that the actual form of the 1-loop corrections also contains terms like

$$
\Delta K_{g_{\mathrm{s}}} \stackrel{?}{=} \frac{t_{b}^{\lambda_{1}} t_{i}^{\lambda_{2}} \mathcal{E}_{i}^{K}}{S_{1} \mathcal{V}}
$$

with $\lambda_{1}+\lambda_{2}=1$ but $0<\lambda_{1} \neq 1$ or 0 , such a 1-loop correction would spoil the large volume expansion performed in (6.16) ${ }^{16}$

\footnotetext{
${ }^{16}$ In principle, one would also need an argument that no such terms arise at higher loop order, which would, however, have to be further suppressed in the dilaton $S_{1}$.
} 


\subsubsection{Fibered Calabi-Yau manifolds}

The feature of orientifolds that all Kähler moduli appear linearly in the cubic expression for the volume shows that a similar simplification can occur in the case of $(K 3)$ fibered Calabi-Yau manifolds, which also have the property that one Kähler modulus (the one corresponding to the volume of the base) only appears linearly in the cubic expression for the volume. This takes the form

$$
\mathcal{V}=t_{b} \eta_{i j} t_{i} t_{j}+d_{i j k} t_{i} t_{j} t_{k}
$$

where $\eta_{i j}$ are the intersection numbers of the (K3) fiber, and neither they nor the triple intersection numbers $d_{i j k}$ contain the index $b$, which is chosen to denote "base", but it is also suggestively the same index as the one we used for the large Kähler modulus in the $\mathbb{P}_{[1,1,1,6,9]}^{4}$ model. Two-parameter examples of this type appear in e.g. [190, 177]. In a region of the Kähler moduli space where the base $t_{b}$ is rather large but all the other $t_{i}$ stay relatively small, the volume is approximately $\mathcal{V}=t_{b} \eta_{i j} t_{i} t_{j}$. Thus, if the Kähler potential has a 1-loop correction $\sim \mathcal{E}_{b}^{K} t_{b} / \mathcal{V}$, it could be approximated in this region by

$$
\frac{\mathcal{E}_{b}^{K} t_{b}}{\mathcal{V}} \sim \frac{\mathcal{E}_{b}^{K}}{\tau_{f}}+\mathcal{O}\left(t_{b}^{-1}\right)
$$

where $\tau_{f}=\eta_{i j} t_{i} t_{j}$ is the volume of the (K3) fiber (which is small compared to $t_{b}^{2}$ ). Obviously, this would lead to a correction to the Kähler potential that is only suppressed by a single (small) 4-cycle volume, similar to the toroidal orientifold example we discussed in the last section.

We should note that this limit (large base and small fiber for $(K 3)$ fibered Calabi-Yau manifolds), is quite different from the one performed in the usual LVS of [169], even though both cases involve hierarchical limits of the Kähler moduli. As explained in section 6.5.1, the LVS limit consists in scaling the overall volume of the Calabi-Yau more or less isotropically while keeping holes in the bulk of the manifold small. In contrast, the limit of large base and small fiber is anisotropic. At the moment we have nothing to add about whether such anisotropic configurations with all moduli stabilized actually exist. We merely wanted to point out that if they do exist, that would be an example of smooth Calabi-Yau compactifications where the $g_{\mathrm{s}}$ corrections we consider dominate over the $\alpha^{\prime}$ corrections considered in the large volume limit, as in the toroidal orientifold case.

\subsection{Further corrections}

In [143], further $\alpha^{\prime}$ corrections to the string effective action beyond the one in (6.9) were considered. In the case of bulk $\alpha^{\prime}$ corrections (i.e. those already present in type IIB bulk theory without D-branes, arising from sphere level) scaling arguments were given 
as to why they are suppressed in the large volume limit. Although that discussion was surprisingly powerful in its simplicity, we do not consider it completely conclusive, if large hierarchies between the Kähler moduli exist. After all, dimensional analysis alone does not guarantee that the other $\alpha^{\prime}$-corrections are always suppressed by additional powers in the overall volume, instead of powers of some of the small Kähler moduli. Moreover, in addition to the bulk $\alpha^{\prime}$ corrections that appear at order $\mathcal{O}\left(\alpha^{\prime 3}\right)$, in the models of interest for LVS further $\alpha^{\prime}$-corrections arise on the worldvolume of D-branes and O-planes, cf. [191, 192, 193, 194, 195, 196, 197, 198. These corrections begin already at order $\mathcal{O}\left(\alpha^{\prime}\right)$ and scaling arguments of the kind used for the bulk corrections do not seem to be sufficient to neglect them.

Indeed, there are correction terms involving two powers of the Riemann tensor which do modify the effective D3-brane charge and tension, if the D7-branes are wrapped over 4cycles with non-vanishing Euler number. These terms were already taken into account in 8. However, there are further contributions to the DBI action at the same order in $\alpha^{\prime}$, like $F_{3}^{2} R$ or $F_{3}^{4}$, where $F_{3}$ stands for the RR 3-form field strength, $R$ for the Riemann tensor and we left index contractions unspecified. If the D7-branes do not break supersymmetry and remain BPS, it seems unlikely that these terms could contribute to the potential for the closed string moduli, i.e. induce some effective D3-brane tension. The reason is that there does not seem to be a corresponding term in the Chern-Simons action that could lead to the necessary modification of the effective D3-brane charge at the same time. This could be checked in more detail.

In general, we think that the question of additional corrections to the moduli (Kähler) potential deserves further attention. Here we only outlined some steps in that direction.

\subsection{A summary on string compactifications}

In this chapter, we have investigated whether string loop corrections may impact a) stabilization in the large volume compactification scenario (LVS), and $b$ ) the phenomenology of those scenarios, as manifested in the soft supersymmetry breaking terms. The result is that for the specific class of compactification manifolds considered in LVS, so-called "Swiss cheese" Calabi-Yau manifolds, changes are minuscule. Only if the loop corrections become abnormally large (in the toroidal orientifold case, this can happen if the complex structure is stabilized very large) do they affect LVS. For other classes of manifolds, the corrections may be important. We hasten to add that the detailed expressions for the loop corrections in LVS remain unknown; we have merely tried to infer their scaling with the Kähler moduli from experience in the toroidal orientifold limit. We think it is important to attempt to address this issue, as the string coupling is stabilized at a nonzero value, so the corrections can not be turned off.

We also stress the (to some readers obvious) fact that there remain a host of issues that 
must ultimately be dealt with if one wishes to claim that these are "string compactifications".

- We can not be sure that fluxes do not alter the corrections, since backgrounds with RR and NSNS fluxes are not well understood in string perturbation theory.

- Additional corrections may appear (see section 6.6) that could be equally threatening to LVS as the loop corrections, or worse.

- In [171] only the corrections to the Kähler potential coming from $\mathcal{N}=2$ sectors were determined and we based our generalization on those results. However, there might be interesting corrections coming from the $\mathcal{N}=1$ sectors as well.

- It has not yet been shown that a local Standard Model-like construction can be embedded in the simplest examples like the $\mathbb{P}_{[1,1,1,6,9]}^{4}$ model. If more general models turn out to be needed, one needs to reconsider whether the requisite nonperturbative superpotentials are generated.

- We have largely ignored open string moduli, under the proviso that they are stabilized heavy, as are the dilaton and complex structure moduli.

- The coefficient $A(S, u)$ in the nonperturbative superpotential is generally assumed to be of order 1. It is not known how generic this is.

- All string computations we have discussed were performed in a supersymmetric context. In LVS supersymmetry is broken already before uplift, in the AdS minimum. Supersymmetry breaking directly in string theory is not very well understood [173, 199].

Faced with all these caveats, a pessimist might be inclined to give up. We think we have shown that it is worth considering these issues in detail. Sometimes, an effect one would have thought to be devastating turns out to be as gentle as a summer breeze. 


\section{A The conifold}

In this appendix we describe the singular and the deformed conifold. The purpose is to provide the formulae which are needed in the main text, and in particular in chapter 4 and 5. We follow [108, 200] and we refer to these papers for further details.

\section{A.1 The singular conifold}

The singular conifold is a non-compact Calabi-Yau threefold. In can be defined as a hypersurface in $\mathbb{C}^{4}$. Two sets of complex projective coordinates are particularly useful: one set is denoted by $w_{A}$, with $A=1,2,3,4$; the conifold is then defined by the equation

$$
w_{1} w_{2}-w_{3} w_{4}=0
$$

A second set is denoted by $z_{A}$, for which the defining equation is

$$
\sum_{A=1}^{4}\left(z_{A}\right)^{2}=0 .
$$

The two sets of coordinates are linearly related by

$$
\begin{aligned}
z_{1} & =\frac{1}{2}\left(w_{1}+w_{2}\right), & z_{2} & =\frac{1}{i 2}\left(w_{1}-w_{2}\right), \\
z_{3} & =\frac{1}{2}\left(w_{3}-w_{4}\right), & z_{4} & =\frac{1}{i 2}\left(w_{3}+w_{4}\right),
\end{aligned}
$$

We can also use 6 real coordinates to parameterize the conifold. The metric is then given by

$$
\begin{aligned}
d s_{6}^{2} & =d r^{2}+r^{2} d s_{T^{1,1}}^{2} \\
d s_{T^{1,1}}^{2} & =\frac{1}{9}\left(d \psi+\sum_{i=1}^{2} \cos \theta_{i} d \phi_{i}\right)^{2}+\frac{1}{6} \sum_{i=1}^{2}\left(d \theta_{i}^{2}+\sin ^{2} \theta_{i} d \phi_{i}^{2}\right) .
\end{aligned}
$$

This makes explicit that the singular conifold has a radial direction $r$ and a base parameterized by 5 angular direction $\phi_{1}, \phi_{2}, \theta_{1}, \theta_{2}$ and $\psi$. The base is $T^{1,1}$, i.e. the coset space $\left(S U(2)_{A} \times S U(2)_{B}\right) / U(1)_{R}$ and it is topologically equivalent to $S^{3} \times S^{2}$, as can be 
seen from the pictorial representation in figure A.1. The complex $w_{A}$ coordinates can be expressed in terms of the real coordinates as

$$
\begin{aligned}
& w_{1}=r^{3 / 2} e^{\frac{i}{2}\left(\psi-\phi_{1}-\phi_{2}\right)} \sin \frac{\theta_{1}}{2} \sin \frac{\theta_{2}}{2}, \\
& w_{2}=r^{3 / 2} e^{\frac{i}{2}\left(\psi+\phi_{1}+\phi_{2}\right)} \cos \frac{\theta_{1}}{2} \cos \frac{\theta_{2}}{2}, \\
& w_{3}=r^{3 / 2} e^{\frac{i}{2}\left(\psi+\phi_{1}-\phi_{2}\right)} \cos \frac{\theta_{1}}{2} \sin \frac{\theta_{2}}{2}, \\
& w_{4}=r^{3 / 2} e^{\frac{i}{2}\left(\psi-\phi_{1}+\phi_{2}\right)} \sin \frac{\theta_{1}}{2} \cos \frac{\theta_{2}}{2} .
\end{aligned}
$$

As for all Calabi-Yau manifolds, the conifold's metric is given by the second derivative of a Kähler potential. For the $w_{A}$ coordinates this is

$$
k\left(w_{i}, \bar{w}_{i}\right)=r^{2}=\left(\sum_{i=1}^{4}\left|w_{i}\right|^{2}\right)^{2 / 3} .
$$

To obtain the metric one of the four coordinates $\left\{w_{1}, w_{2}, w_{3}, w_{4}\right\}$, has to be expressed in terms of the other using the defining equation (A.1). If, e.g. we chose to keep $\left\{w_{2}, w_{3}, w_{4}\right\}$ and express $w_{1}$ as a function of them, the inverse metric (appearing in (4.16)) is given by

$$
\begin{aligned}
k^{\bar{\jmath}, i} \equiv & \left(k_{i, \bar{\jmath}}\right)^{-1} \\
= & \frac{3}{2} r\left\{\delta_{i j}+\frac{w_{i} \bar{w}_{j}}{2 r^{3}}-\frac{1}{r^{3}}\left(\begin{array}{cc}
\left|w_{1}\right|^{2} & \left|w_{4}\right|^{2} \\
\left|w_{3}\right|^{2}
\end{array}\right)\right. \\
& \left.+\frac{\left|w_{1}\right|^{2}}{2 r^{3}}\left[\delta_{1 i}\left(\delta_{1 j}-1\right) \frac{\bar{w}_{i}}{\bar{w}_{j}}+\delta_{1 j}\left(\delta_{1 i}-1\right) \frac{w_{i}}{w_{j}}\right]\right\} \\
= & \frac{3}{2 r^{2}}\left(\begin{array}{ccc}
r^{3}-\left|w_{1}\right|^{2}+\frac{1}{2}\left|w_{2}\right|^{2} & \frac{w_{3}}{w_{2}}\left(\left|w_{2}\right|^{2}+2\left|w_{4}\right|\right)^{2} & \frac{w_{4}}{w_{2}}\left(\left|w_{2}\right|^{2}+2\left|w_{3}\right|\right)^{2} \\
\frac{\bar{w}_{3}}{\bar{w}_{2}}\left(\left|w_{2}\right|^{2}+2\left|w_{4}\right|\right)^{2} & r^{3}-\left|w_{4}\right|^{2}+\frac{1}{2}\left|w_{3}\right|^{2} & w_{4} \bar{w}_{3} \\
\frac{w_{4}}{\bar{w}_{2}}\left(\left|w_{2}\right|^{2}+2\left|w_{3}\right|\right)^{2} & w_{3} \bar{w}_{4} & r^{3}-\left|w_{3}\right|^{2}+\frac{1}{2}\left|w_{4}\right|^{2}
\end{array}\right),
\end{aligned}
$$

where $i$ and $j$ run from 1 to 3 .

\section{A.2 The deformed conifold}

In this appendix we review some useful facts about the deformed conifold. At the end we will obtain an expression for $\partial_{r} z_{1}$ at the tip, which is what we need to check the radial stability in appendix C.2. We restrict ourselves to the $z_{A}$ coordinates. 


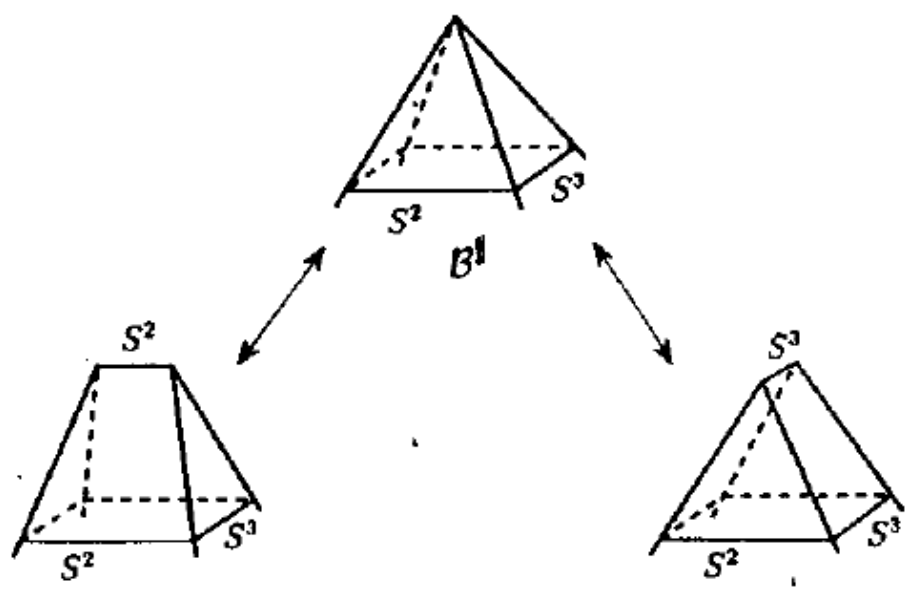

Figure A.1: The deformed, resolved and singular conifold are depicted. Figure taken from [108].

The deformed conifold is defined by the following hypersurface in $\mathbb{C}^{4}$

$$
\sum_{A=1}^{4}\left(z_{A}\right)^{2}=\varepsilon^{2}
$$

where $\varepsilon$ is a complex parameter. When $\varepsilon=0$, A.12 reduces to A.2), i.e. the singular conifold. In terms of the matrix $W=\frac{1}{\sqrt{2}}\left(z_{i} \sigma_{i}+i z_{4}\right)$, where $\sigma_{i}$ are the Pauli matrices, this expression can be written as

$$
\operatorname{det} W=-\frac{\varepsilon^{2}}{2}
$$

A radial coordinate can be defined by

$$
\sum_{A=1}^{4}\left|z_{A}\right|^{2}=\operatorname{tr}\left(W W^{\dagger}\right)=r^{3} .
$$

A generic solution to A.12 can be written as

$$
W=L_{1} Z_{0} L_{2}^{\dagger}
$$

where $L_{1}, L_{2}$ are $S U(2)$ matrices that can be parameterized using Euler angles

$$
L_{j}=\left(\begin{array}{cc}
\cos \frac{\theta_{j}}{2} e^{i\left(\psi_{j}+\phi_{j}\right) / 2} & -\sin \frac{\theta_{j}}{2} e^{-i\left(\psi_{j}-\phi_{j}\right) / 2} \\
\sin \frac{\theta_{j}}{2} e^{i\left(\psi_{j}-\phi_{j}\right) / 2} & \cos \frac{\theta_{j}}{2} e^{-i\left(\psi_{j}+\phi_{j}\right) / 2}
\end{array}\right)
$$

and

$$
\begin{aligned}
Z_{0} & =\left(\begin{array}{ll}
0 & a \\
b & 0
\end{array}\right), \\
a & =\frac{1}{2}\left(\sqrt{r^{3}+\varepsilon^{2}}+\sqrt{r^{3}-\varepsilon^{2}}\right), \quad b=\frac{\varepsilon^{2}}{2 a} .
\end{aligned}
$$


For $r^{3 / 2}>\varepsilon$, one of the six angles $\left\{\phi_{i}, \psi_{i}, \theta_{i}\right\}, i=1,2$, is redundant. We can fix the gauge imposing $\psi_{2}=0$; we obtain the following real parameterization of the complex coordinates $z_{A}$

$$
\begin{aligned}
z_{1}= & \frac{1}{\sqrt{2}}\left(a \cos \frac{\theta_{1}}{2} \cos \frac{\theta_{2}}{2} e^{i\left(\psi_{1}+\phi_{1}+\phi_{2}\right) / 2}-b \sin \frac{\theta_{1}}{2} \sin \frac{\theta_{2}}{2} e^{-i\left(\psi_{1}-\phi_{1}-\phi_{2}\right) / 2}\right. \\
& \left.-a \sin \frac{\theta_{1}}{2} \sin \frac{\theta_{2}}{2} e^{i\left(\psi_{1}-\phi_{1}-\phi_{2}\right) / 2}+b \cos \frac{\theta_{1}}{2} \cos \frac{\theta_{2}}{2} e^{-i\left(\psi_{1}+\phi_{1}+\phi_{2}\right) / 2}\right), \\
z_{2}= & \frac{i}{\sqrt{2}}\left(a \cos \frac{\theta_{1}}{2} \cos \frac{\theta_{2}}{2} e^{i\left(\psi_{1}+\phi_{1}+\phi_{2}\right) / 2}-b \sin \frac{\theta_{1}}{2} \sin \frac{\theta_{2}}{2} e^{-i\left(\psi_{1}-\phi_{1}-\phi_{2}\right) / 2}\right. \\
& \left.+a \sin \frac{\theta_{1}}{2} \sin \frac{\theta_{2}}{2} e^{i\left(\psi_{1}-\phi_{1}-\phi_{2}\right) / 2}-b \cos \frac{\theta_{1}}{2} \cos \frac{\theta_{2}}{2} e^{-i\left(\psi_{1}+\phi_{1}+\phi_{2}\right) / 2}\right), \\
z_{3}= & -\frac{1}{\sqrt{2}}\left(a \cos \frac{\theta_{1}}{2} \sin \frac{\theta_{2}}{2} e^{i\left(\psi_{1}+\phi_{1}-\phi_{2}\right) / 2}+b \sin \frac{\theta_{1}}{2} \cos \frac{\theta_{2}}{2} e^{-i\left(\psi_{1}-\phi_{1}+\phi_{2}\right) / 2}\right. \\
& \left.-a \sin \frac{\theta_{1}}{2} \cos \frac{\theta_{2}}{2} e^{i\left(\psi_{1}-\phi_{1}+\phi_{2}\right) / 2}-b \cos \frac{\theta_{1}}{2} \sin \frac{\theta_{2}}{2} e^{-i\left(\psi_{1}+\phi_{1}-\phi_{2}\right) / 2}\right), \\
z_{4}= & \frac{i}{\sqrt{2}}\left(-a \sin \frac{\theta_{1}}{2} \cos \frac{\theta_{2}}{2} e^{i\left(\psi_{1}-\phi_{1}+\phi_{2}\right) / 2}-b \cos \frac{\theta_{1}}{2} \sin \frac{\theta_{2}}{2} e^{-i\left(\psi_{1}+\phi_{1}-\phi_{2}\right) / 2}\right. \\
& \left.a \cos \frac{\theta_{1}}{2} \sin \frac{\theta_{2}}{2} e^{i\left(\psi_{1}+\phi_{1}-\phi_{2}\right) / 2}+b \sin \frac{\theta_{1}}{2} \cos \frac{\theta_{2}}{2} e^{-i\left(\psi_{1}-\phi_{1}+\phi_{2}\right) / 2}\right) .
\end{aligned}
$$

The singular conifold, i.e. $\varepsilon=0$, is just the special case $a=r^{3 / 2}, b=0$. Now we want to calculate the derivative of $z_{1}$ with respect to $r$ evaluated at the tip $r=\varepsilon^{2 / 3}$. At the tip, three of the six Euler angles are redundant, a possible choice of the gauge is $\theta_{2}=\psi_{2}=\phi_{2}=0$. The result is then

$$
\begin{aligned}
\lim _{r \rightarrow \varepsilon^{2 / 3}} \operatorname{Im} \frac{\partial z_{1}}{\partial r} & =\frac{\sqrt{6}}{4} \frac{\cos \frac{\theta_{1}}{2} \sin \frac{\psi_{1}+\phi_{1}}{2} \varepsilon^{2 / 3}}{\sqrt{r-\varepsilon^{2 / 3}}}+\mathcal{O}(1) \\
\lim _{r \rightarrow \varepsilon^{2 / 3}} \operatorname{Re} \frac{\partial z_{1}}{\partial r} & =\frac{3}{4} \varepsilon^{1 / 3} \cos \frac{\theta_{1}}{2} \cos \frac{\psi_{1}+\phi_{1}}{2}+\mathcal{O}\left(r-\varepsilon^{2 / 3}\right) \\
& =\frac{3}{4} \varepsilon^{1 / 3} E\left(\theta_{1}, \phi_{1}, \psi_{1}\right)+\mathcal{O}\left(r-\varepsilon^{2 / 3}\right)
\end{aligned}
$$

where in the last line we have introduced the function $\left|E\left(\theta_{1}, \phi_{1}, \psi_{1}\right)\right|<1$ for further reference. We see that the imaginary part of the derivative is singular but the real part has a finite non vanishing value. 


\section{B Technical details on radial brane inflation}

In the following appendices we deal with a series of technical details related to the radial brane inflation model constructed in chapter 4. Appendix B.1 analyzes the dependence of the stabilized volume modulus $\tau_{c}$ on the uplifting and on the inflaton. Appendix B.2 shows that the coefficient of the $\phi^{3 / 2}$ term in the inflaton potential (4.58), for the Kuperstein embedding, is non-positive. This feature determines the general structure of the potential which is derived in Appendix B.3.

\section{B.1 Dependence of $\tau_{c r}$ on the uplifting and the inflaton}

In this appendix, we discuss the minimization of the volume in the model of chapter 4 . In particular we derive its dependence on the uplifting parameter and on the inflaton. As explained in section 4.4 .2 and shown graphically in figure 4.2 , the latter dependence is crucial for a correct inflationary analysis.

The $\mathrm{N}=1$, 4-dimensional F-term potential

$$
V_{\mathrm{AdS}}=\frac{a A_{0} e^{-a \tau}}{2 \tau^{2}}\left(\frac{1}{3} \tau a A_{0} e^{-a \tau}+W_{0}+A_{0} e^{-a \tau}\right)
$$

resulting from the choice

$$
\begin{aligned}
K & =-3 \log (T+\bar{T}) \\
W & =W_{0}+A_{0} e^{-a T}
\end{aligned}
$$

has a well known [20] AdS minimum $\tau_{0}$ (see section 2.6 and figure 2.4), solution of the transcendental equation

$$
\tau_{0}=-\frac{3}{2} \frac{A_{0}+W_{0} e^{a \tau_{0}}}{a A_{0}}
$$

where $W_{0}$ is a negative real number. The aim of this appendix is to calculate how this minimum changes when an uplifting and a D3-brane are present as in the brane inflation model of chapter 4 . 


\section{B.1.1 On $\tau_{c r}$ and the uplifting}

For concreteness, we look at an uplifting generated by the warped tension of an anti D3-brane. In the type IIB setup with imaginary self-dual fluxes of section 2.5.1 [8], an anti D3-brane feels a potential that pulls it to regions with strong warping. If a warped throat is present, the anti D3-brane will stabilize at its tip. Supersymmetry is broken and the effective scalar potential receives a contribution proportional to the redshifted anti D3-brane tension that we schematically indicate as

$$
V_{\text {up }}=\frac{D}{4 \tau^{2}}
$$

The AdS minimum $\tau=\tau_{0}$ in $(\overline{B .4})$ is shifted and uplifted. We call the new minimum $\tau_{u p}$ and define the shift $\Delta_{\beta} \equiv \tau_{u p}-\tau_{0}$, induced by the uplifting term. It is useful to trade the parameter $D$ for another parameter $\beta$, rewriting it as

$$
D=\beta \frac{2}{3} \tau_{0} a^{2} A_{0}^{2} e^{-2 a \tau_{0}} .
$$

The condition that $V_{\text {up }}$ uplifts the AdS minimum to dS is now easily expressed by the requirement $\beta \gtrsim 1+2 \Delta / \tau_{0}$ (which is very close to, but not exactly one). In what follows, we make the hypothesis that this condition is fulfilled and therefore the minimum is dS. The equation $\partial_{\tau}\left(V_{\text {AdS }}+V_{\text {up }}\right)=0$ is equivalent to

$$
\tau_{u p}=-\frac{1}{4 a A_{0}}\left(7 A_{0}+3 W_{0} e^{a \tau_{u p}}-\sqrt{\left(A_{0}-3 W_{0} e^{a \tau_{u p}}\right)^{2}-96 D e^{a \tau_{u p}} / a}\right) .
$$

As it was the case for (B.4), this is a transcendental equation and has to be solved numerically. To get some analytical control, we use the following trick. We substitute $D$ and $W_{0}$ in (B.7) using (B.6) and (B.4). Then we solve the resulting equation for $\Delta$ in terms of $\tau_{0}$ and the others parameters. This can be done expanding $e^{a \Delta} \simeq 1+a \Delta$ so that the equation is no more transcendental. At the end of the day the only transcendental equation that we have to solve numerically is B.4. and we have an analytical expression for $\Delta$.

The expression for $\Delta$ is a little bit long, so we write its expansion in $1 / a \tau_{0}$, this is

$$
\Delta_{\beta} \simeq \frac{\beta}{a^{2} \tau_{0}}+\frac{\beta(4 \beta-5)}{2 a^{3} \tau_{0}^{2}}+\ldots
$$

in very good agreement with the numerical calculation. We notice that this is equivalent to an expansion in $D /\left(a W_{0}^{2}\right)$ of (B.7); in fact from (B.6) one sees that $D$ is suppressed with respect to $W_{0}^{2}$ by a factor $1 / \tau_{0}$. This expansion would give the transcendental equation

$$
\tau_{u p}=-\frac{3}{2} \frac{A_{0}+W_{0} e^{a \tau_{u p}}}{a A_{0}}-\frac{12 D e^{2 a \tau_{u p}}}{a^{2} A_{0}\left(A_{0}-3 W_{0} e^{a \tau_{u p}}\right)}+\ldots
$$




\section{B.1.2 On $\tau_{c r}$ and the inflaton}

In this section we take into account also a dynamical D3-brane and calculate its effect on the minimum of the potential in the $\tau$ direction that we call $\tau_{c r}$. The potential is given in (4.39). We expand $\partial_{\tau} V=0$ for small $\mathrm{r}$ (again this is an $r^{2} / \tau$ or an $r^{3 / 2} /(\mu n)$ expansion). Solving for $\tau_{c r}$ one gets

$$
\begin{aligned}
\tau_{c r}(D, r)= & \tau_{c r}(r=0)+\tau_{c r}^{(1)} r+\tau_{c r}^{(3 / 2)} r^{3 / 2}+\ldots \\
= & \tau_{c r}(r=0)+\frac{9\left(A_{0}+3 W_{0} e^{a \tau_{c r}}\right)}{8 a^{2} \mu^{2} n^{2} \gamma\left(A_{0}-3 W_{0} e^{a \tau_{c r}}\right)} r \\
& -\frac{3\left(A_{0}^{2}+2 A_{0}^{2} W_{0} e^{a \tau_{c r}}+3 W_{0}^{2} e^{2 a \tau_{c r}}\right)}{2 a A_{0} \mu n\left(A_{0}-3 W_{0} e^{a \tau_{c r}}\right)} r^{3 / 2}+\ldots
\end{aligned}
$$

where $\tau_{c r}(r=0)$ is the one in $(\mathrm{B} .9)$ and in $\tau_{c r}^{(1)}$ and $\tau_{c r}^{(3 / 2)}$ we have neglected terms suppressed by a factor of order $D / W_{0}^{2}$ (see (B.6)). As we did in the last section we substitute $D$ and $W_{0}$ in (B.7) using (B.6) and (B.4). Then we solve for $\Delta_{r}=\tau_{c r}-\tau_{c r}(r=$ $0)$. The result is

$$
\Delta_{r}=\frac{r^{3 / 2}}{a \mu n}+\ldots
$$

To summarize, we have estimated analytically the dependence of the minimum on the uplifting and on the D3-brane position; this is given, at leading order, by

$$
\begin{aligned}
\tau_{c r} & =\tau_{0}+\Delta_{\beta}+\Delta_{r} \\
& \simeq \tau_{0}+\frac{\beta}{a^{2} \tau_{0}}+\frac{r^{3 / 2}}{a \mu n}+\ldots
\end{aligned}
$$

\section{B.2 Sign of $r^{3 / 2}$ term}

In this appendix we show that the expansion of the scalar potential has a negative term at order $r^{3 / 2}$. This term determines the negative curvature of the potential for small $r$. In fact in (4.57) there is also a term proportional to $r$, but of course it does not contribute to the curvature.

The explicit values of $V_{\mathrm{dS}}^{(3 / 2)}$ and $\Delta V^{(3 / 2)}$ are

$$
\begin{aligned}
V_{\mathrm{dS}}^{(3 / 2)} & =-\frac{\left[3 a A_{0}^{2} e^{-2 a \tau_{0}}\left(a \tau_{c r}+6\right)+D+18 A_{0} a W_{0} e^{-a \tau_{0}}\right]}{18 a \mu n \tau_{0}^{3}}, \\
\Delta V^{(3 / 2)} & =-\frac{A_{0}^{2} a e^{-2 a \tau_{0}}}{4 \mu n \tau_{0}^{2}}
\end{aligned}
$$




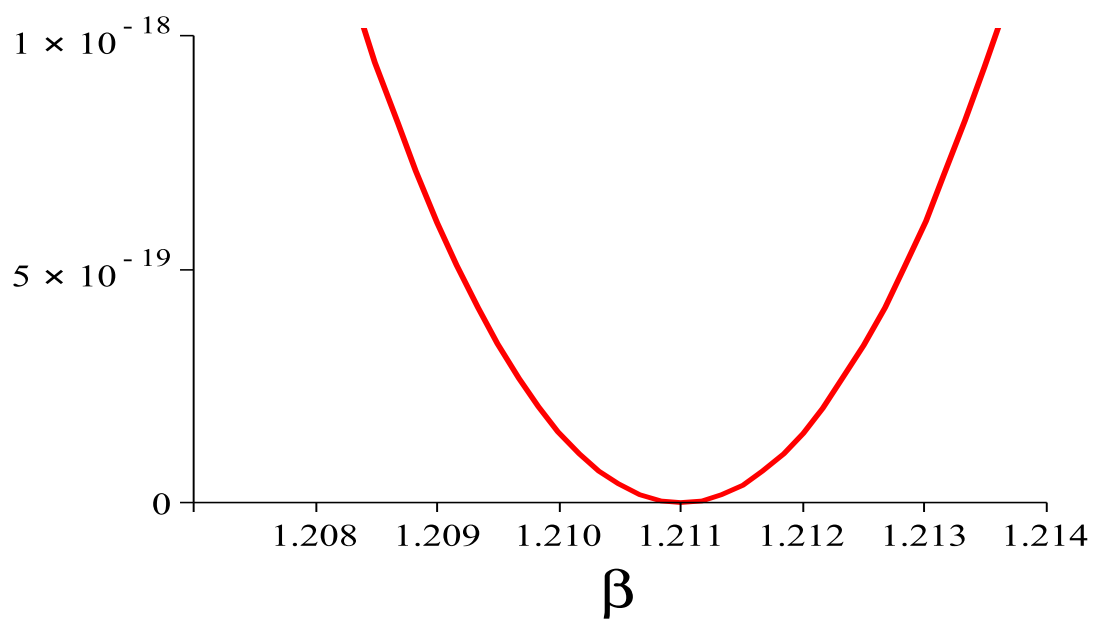

Figure B.1: The plot shows the discriminant (B.21) as a function of $\beta$ (without performing the $\Delta / \tau_{0}$ expansion). When the discriminant is zero minimum and maximum of the potential coincide and we get a inflection point.

To see that $V^{(3 / 2)}=V_{\mathrm{dS}}^{(3 / 2)}+\Delta V^{(3 / 2)}<0$ we substitute $\left.\mathrm{B} .6\right)$ and $(\mathrm{B} .4$ in (B.13) and expand in $\tau_{u p}-\tau_{0}=\Delta_{\beta}$. This gives

$$
V_{\mathrm{dS}}^{(3 / 2)} \simeq \frac{A_{0}^{2} a e^{-2 a \tau_{0}}(3-2 \beta)}{6 \mu n \tau_{0}^{2}}+\ldots
$$

Therefore

$$
\frac{V_{\mathrm{dS}}^{(3 / 2)}}{\Delta V^{(3 / 2)}} \simeq-\frac{4(3-2 \beta)}{6} \gtrsim-1 / 2
$$

for $\beta \gtrsim 1$. We are thus left with

$$
V^{(3 / 2)}=-\frac{A_{0}^{2} a e^{-2 a \tau_{0}}}{12 \tau_{0}^{2} \mu n}(4 \beta-3)<0
$$

in agreement with (B.19).

\section{B.3 Maximum and minimum of $V(\phi)$}

In this section, we show that in the case of the Kuperstein embedding, the effective potential (4.58) (where we neglect the Coulomb term because it is subleadin as discussed in section 4.4.4) in the $r$ (or equivalently $\phi$ ) direction has always a maximum and a 
minimum (in the extreme case they coincide). Our starting point is

$$
\begin{aligned}
V(\phi)= & \frac{A_{0}^{2} a^{2} e^{-2 a \tau_{0}}}{6 \tau_{0}}(\beta-1)+\phi \frac{9 A_{0}^{2} e^{-2 a \tau_{0}} M_{P l}^{2}}{16 T_{D 3}^{3 / 2} \tau_{0}^{3 / 2} \mu^{2} n^{2}} \\
& -\phi^{3 / 2} \frac{A_{0}^{2} a e^{-2 a \tau_{0}}}{12 \tau_{0}^{5 / 4} T_{D 3}^{3 / 4} \mu n}(4 \beta-3)+\frac{\phi^{2}}{3 M_{P l}^{2}} \frac{A_{0}^{2} a^{2} e^{-2 a \tau_{0}}}{6 \tau_{0}}(\beta-1),
\end{aligned}
$$

which is the potential 4.58 with

$$
\begin{aligned}
\Lambda=\frac{3}{M_{P l}^{2}} C_{2} & =\frac{A_{0}^{2} a^{2} e^{-2 a \tau_{0}}}{6 \tau_{0}}(\beta-1), \\
C_{1} & =\frac{9 A_{0}^{2} e^{-2 a \tau_{0}} M_{P l}^{2}}{16 T_{D 3}^{3 / 2} \tau_{0}^{3 / 2} \mu^{2} n^{2}} \\
C_{3 / 2} & =\frac{A_{0}^{2} a e^{-2 a \tau_{0}}}{12 \tau_{0}^{5 / 4} T_{D 3}^{3 / 4} \mu n}(4 \beta-3) .
\end{aligned}
$$

The first derivative of the potential is a quadratic polynom in $\sqrt{\phi}$. There are two extrema (a maximum and a minimum) when the discriminant is positive, i.e. $9 C_{3 / 2}-32 C_{1} C_{2}>0$. Explicitly (see figure B.1)

$$
9 C_{3 / 2}-32 C_{1} C_{2}=\frac{A_{0}^{2} a^{2} e^{-2 a \tau_{0}}}{64 \tau_{0}^{5 / 2} T_{D 3}^{3 / 2} \mu^{2} n^{2}}(4 \beta-5)^{2} .
$$

This quantity is always positive so that $V(\phi)$ will always have a minimum and a maximum. Also it is evident that for a precise value of $\beta$ the discriminant becomes zero. This indicates that maximum and minimum coincide, in other words there is a flat inflection point. We have plotted the discriminant (without expanding it) in figure B.1. This confirms the result of our leading order calculation.

\section{B.4 Radial brane inflation close to the tip}

In chapter 4, we have considered the possibility to cancel the large inflaton mass term using the threshold corrections to the non perturbative superpotential. As reviewed in section 4.3, large mass for the inflaton is induced by the mixing of the radial position of the D3-brane and the Kähler moduli. This comes about if one considers the scalar potential

$$
\begin{aligned}
V(\tau, \phi) \simeq & V_{K K L T}+V_{u p} \\
\simeq & \frac{2 \kappa_{4}^{2} A_{0} a e^{-a \tau}}{U(r)^{2}}\left\{A_{0} e^{-a \tau}-\left|W_{0}\right|+\right. \\
& \left.\quad+\frac{1}{6}\left[T+\bar{T}+\gamma\left(k_{i} k^{\overline{i j} j} k_{j}-k\right)\right] A_{0} a e^{-a \tau}\right\}+\frac{D}{U(r)^{2}}
\end{aligned}
$$


Far away from the tip but still inside the throat, where the deformed conifold geometry is well described by the singular conifold metric, we have (see appendix A)

$$
\begin{aligned}
k(z, \bar{z}) & =\left(\sum_{A=1}^{4}\left|z_{A}\right|^{2}\right)^{2 / 3}=r^{2} \Rightarrow \quad k_{\bar{i}} k^{\bar{i} j} k_{j}=k=r^{2} \\
U & =2 \tau-\gamma k(z, \bar{z})=2 \tau-\gamma r^{2} .
\end{aligned}
$$

Expanding (B.22) in $r^{2} \ll \tau$ one obtains a term $r^{2}$ that induces a slow-roll parameter $\eta$ of order one and prevents radial slow-roll inflation. The result of the investigation of chapter 4 (based on [64], but see also [68, 66]) is that this mass term can not be cancelled for a large range of the radial position because the threshold corrections to $W$ produce only terms like $r, r^{3 / 2}, r^{3}, r^{9 / 2} \ldots$

Here we notice that the situation is different if one consider the motion of the D3-brane close to the tip of the deformed conifold. There the deformed conifold Kähler potential is well approximated by

$$
\begin{aligned}
k(z, \bar{z}) & =\tilde{k}_{0}+\sum_{A=1}^{4}\left|z_{A}\right|^{2} \equiv \tilde{k}_{0}+r^{3} \quad \Rightarrow \quad k_{i} k^{i j} k_{j}=0 \\
U & =2 \tau-\gamma k(z, \bar{z})=2 \tau-\gamma\left(\tilde{k}_{0}+r^{3}\right)
\end{aligned}
$$

for some constant $\tilde{k}_{0}$. Substituting this in $\left(\mathrm{B} .22\right.$ and expanding in $r^{3} \ll \tau$, we see that near the tip the moduli stabilization induces a cubic term and not a quadratic one as it is the case away from the tip.

Therefore, a cancellation between the threshold corrections to the nonperturbative superpotential and the term induced by the moduli stabilization is in principle possible. This cancellation would happen only close to the tip where the approximated form of the Kähler potential (B.24) is valid, hence it interests only a short range of the radial position. Hence again, even allowing for fine tuning, one can flatten the potential only in a small region, analogously to the case considered in chapter 4.

\section{B.5 Forces on D3- and anti D3-branes.}

In this appendix we enumerate the contributions to the potential for an anti D3- and a D3-brane (a sketch is given in table B.1 below) and comment on their relative importance.

To summarize: the anti D3-brane is led to the tip $(r \simeq 0)$ by the interaction with the background; there its angular position is determined by the bulk and moduli stabilization effects. The motion of the D3-brane is governed by moduli stabilization effects (breaking of the no-scale structure). Finally the Coulomb attractive potential is generically very suppressed $\left(a_{0}^{4}\right)$ and plays a role only in very fine tuned or symmetric circumstances.

\footnotetext{
${ }^{1}$ Or no terms at all, depending on the particular embedding chosen.
} 
Background effects. We consider the action

$$
S_{\overline{D 3} / D 3}=-T_{D 3} \int d^{4} x \sqrt{-g} \Phi_{ \pm}
$$

where $\Phi$ is defined in term of the warp factor and of the 5 form field strength of $C_{4}$ as

$$
\Phi_{ \pm} \equiv e^{4 A} \pm \alpha
$$

In the GKP setup [8] reviewed in subsection 2.5.1, we found that the equations of motion require $\alpha=e^{4 A}$. Hence a D3-brane doesn't feel any force (it is BPS with respect to the background). On the contrary, an anti D3-brane tends to fall to the bottom of the (deformed) conifold (small warp factor) to minimize $S_{\overline{D 3}}$. As $(\overline{B .25})$ has no angular dependence, the anti D3-brane at the tip enjoys a translational $S^{3}$ symmetry (that will be broken by bulk effects as we will discuss). The leading contribution of (B.25) to the potential is the warped anti D3-brane tension which can be used [20] to break supersymmetry and uplift an AdS vacuum to a dS one.

Bulk effects. To have a compact manifold at a certain radius the conifold has to be cut and glued to a compact Calabi-Yau manifold. Then other "bulk" effects for the anti D3brane arise. They break all the residual symmetry of the conifold as Calabi-Yau manifolds have no continuous symmetry. In [201] the warp factor dependence of bulk effects has been calculated via AdS/CFT. The result is that a mass for the anti D3-brane is induced of order

$$
m_{\text {bulk }}^{2} \sim\left(g_{s} M \alpha^{\prime}\right)^{-1} a_{0}^{3.29}
$$

where $M$ is the flux quantum number of the Ramond-Ramond $F_{3}$-form over the 3 -cycle $A$ of the throat. Bulk effects would lead the anti D3-brane to a particular angular position in the $S^{3}$ at the tip. No such effects are present for the D3-brane, again because of its BPS nature with respect to the background. This raises the suspicion that the tip might be an interesting place for inflation with a D3-brane. We dedicate chapter 5 to verify this suspicion.

Moduli stabilization effects. To stabilize the Kähler moduli, one has to break the noscale structure. Once this is done and the moduli are stabilized (e.g. à la KKLT) a mass for the D3-brane moduli is generated because of their non-trivial mixing with the Kähler moduli[80]. The potential generated gives rise to the $\eta$-problem we reviewed in section 4.3 .

As regards the anti D3-brane, these effects have been investigated in [75]. They are relevant at the tip of the throat because the background force, coming from (B.25), does not have an angular dependence. The potential generated by the stabilization of the 


\begin{tabular}{lll} 
Source & $\overline{D 3}$ & $D 3$ \\
\hline \hline Bulk background & $m_{b u l k}^{2} \sim\left(g_{s} M \alpha^{\prime}\right)^{-1} a_{0}^{3.29}$ & no effect (BPS) \\
Throat background & $V \sim 2 T_{\overline{D 3}} h\left(w_{\overline{D 3}}\right)$ & no effect (BPS) \\
& leads $\overline{D 3}$ quickly to the tip & \\
Coulomb & $+V_{D \bar{D}}$ & $-V_{D \bar{D}}$ \\
Tachyon & develops at $r^{2}=\mathcal{O}\left(\alpha^{\prime}\right)$ & develops at $r^{2}=\mathcal{O}\left(\alpha^{\prime}\right)$ \\
Moduli stabilization & only known at the tip & $V_{\text {KKLT }}+V_{\text {up }}+\Delta V$
\end{tabular}

Table B.1: Contributions to the potential for an anti D3 and a D3 brane.

moduli has the same minima for the D3-brane and for the anti D3-brane at the tip. The equations for the minimum for a D3- and an anti D3-brane differ by a term vanishing at the tip, therefore away from it, the respective minima will be generically different.

This effect together with the bulk effect (B.27), select some vacua in the angular direction at the tip. The relative importance of bulk and stabilization effects depends on the parameters. Comparing the mass from the lhs of (4.33) ) with (B.27) ) for the case of the Ouyang (or the simplest Kuperstein) embedding, expanding in $r_{0}^{3 / 2} / \mu \ll 1$, one gets

$$
\frac{r_{0}^{3 / 2}}{\mu n} \gg a_{0}^{1.29}
$$

where $r_{0}$ indicates the tip of the deformed conifold. As follows from (4.21)), the lhs of (B.28)) has to be much smaller than one to allow to integrate out the stabilized volume and use the remaining effective potential for inflation. Indeed $r_{0}$ and $a_{0}$ are related by $a_{0} \sim r_{0} / \sqrt{g_{s} M}$, so that the condition to satisfy is

$$
g_{s} M \gg \frac{(\mu n)^{3 / 2}}{r_{0}^{1 / 3}} .
$$

Although it is possible to fulfil the inequality, this could require a very large flux number $M$, as $g_{s} \ll 1$ and $\mu n \gg 1$.

Coulomb potential. The Coulomb potential, written in term of canonically normalized fields, is

$$
V_{D 3 \bar{D} 3} \simeq-\frac{4 \pi^{2} \phi_{0}^{4}}{N}\left(1-\frac{1}{N} \frac{\phi_{0}^{4}}{\phi^{4}}\right)
$$

where $N=K M$ is the product of the fluxes on the three cycles of the conifold (see the discussion around (5.34) ). This potential can be obtained considering the backreaction of a D3-brane on the metric $(\mathrm{B} .25)$ and keeping the leading order term. 


\section{Technical details on angular brane inflation}

In this series of appendices we collect some technical details about the inflation at the tip model. In appendix C.1 we discuss the minimization of the overall volume (Kähler modulus). In appendix C.2 we check the stability of the radial direction for a D3-brane at the tip; in this regard, we stress the role played by the uplifting term. Finally, in appendix C.3, we generalize the form of the Kähler potential and the superpotential in the presence of many D3-branes.

\section{C.1 Minimization of $\tau$}

In this section, we minimize the potential (5.13) along the volume direction. This is, for inflation at the tip, the anologous of what we did in appendix B.1 for the radial brane inflation model. The critical volume $\tau_{c r}$ is a function of the inflaton (the position of the D3-brane at the tip). Contrary to the radial brane inflation case, here this dependence can safely be neglected if we are interested in the leading order single-field effective inflaton potential (see (C.5)). The KKLT minimum for $\tau$ [20] is shifted both by the uplifting and by the presence of the D3-brane. Here we provide an analytical estimate along the lines of appendix B.1 (based on [64]). We call $\tau_{0}$ the minimum of the KKLT potential with

$$
W_{0}=-A_{0} e^{-a \tau_{0}}\left[1+\frac{1}{3} a\left(2 \tau_{0}-k_{0}\right)\right] .
$$

With $\tau_{c r}$ we denote the actual minimum of $(5.13)$ and with $\Delta$ the shift due to the uplifting and the D3-brane: $\Delta=\tau_{c r}-\tau_{0}$. We parameterize the uplifting as

$$
V_{u p}=\frac{D}{U^{b}}=\beta \frac{\left|A_{0}\right|^{2} a^{2} e^{-2 a \tau_{0}}\left(2 \tau_{0}-k_{0}\right)^{b-1}}{3 U^{b}},
$$

where $\beta \gtrsim 1$ gives a de Sitter space. We substitute these expressions for $W_{0}$ and $D$ into the potential (5.13) and take the derivative with respect to $\tau$. We substitute $\tau=\tau_{0}+\Delta$ in $V^{\prime}=0$ and solve for $\Delta$. At leading order the result is given by

$$
\Delta=\frac{b}{2} \frac{\beta}{a^{2} \tau_{0}}-\frac{\varepsilon}{a \mu n} \cos \phi+\frac{\varepsilon^{2 / 3}}{2 c a^{3} n^{2} \mu^{2} \tau_{0} \gamma} \sin ^{2} \phi
$$


In the particular case $b=2$, the shift due to the uplifting reproduces the result of appendix B.1 [64, 68 and the first of the two $\phi$ dependent terms in (C.3) is analogous to the dependence on the radial motion in (B.11). We define $\tau_{u p} \equiv \tau_{0}+b \beta /\left(2 a^{2} \tau_{0}\right)$, which would be the minimum of the volume in the presence of an uplifting term but without the D3-brane. Finally the critical value of the Kähler modulus is

$$
\tau_{c r}(\phi) \simeq \tau_{u p}-\frac{\varepsilon}{a \mu n} \cos \phi+\frac{\varepsilon^{2 / 3}}{2 c a^{3} n^{2} \mu^{2} \tau_{0} \gamma} \sin ^{2} \phi
$$

We use this result to prove that the dependence of $\tau_{c r}$ on $\phi$ is mild in the sense that it produces in the effective potential $V\left(\tau_{c r}(\phi), \phi\right)$ only subleading terms. We substitute $W_{0}$ from (C.1), $D$ from (C.2) and $\tau_{c r}$ from (C.4) into $V\left(\tau_{c r}(\phi), \phi\right)-V\left(\tau_{u p}, \phi\right)$. We keep the leading terms in the $\varepsilon / \mu$ and large volume expansion, the result is

$$
\begin{aligned}
V\left(\tau_{c r}(\phi), \phi\right)-V\left(\tau_{u p}, \phi\right) \simeq & \frac{\left|A_{0}\right|^{2} \varepsilon^{5 / 3} e^{-2 a \tau_{0}}}{6 c n^{3} \mu^{3} \tau_{0}^{2} \gamma} \cos \phi \sin ^{2} \phi+ \\
& -\frac{\left|A_{0}\right|^{2} a^{2} \varepsilon^{2} e^{-2 a \tau_{0}}}{6 n^{2} \mu^{2} \tau_{0}} \cos ^{2} \phi+\ldots
\end{aligned}
$$

Comparing these term with those in $(5.23),(5.24)$ and $(5.25)$, we see that they are subleading in $\varepsilon / \mu$ or in the large volume expansion. We conclude therefore that at leading order $V\left(\tau_{c r}(\phi), \phi\right) \simeq V\left(\tau_{u p}, \phi\right)$, i.e. we are allowed to neglect the $\phi$ dependence of $\tau_{c r}$. This justify the use of (5.16) or (5.18) as leading order potential.

We stress again that this result is very different from the radial brane inflation model. There we showed that the inflaton dependence of $\tau_{c r}$ changes even qualitatively the shape of the inflaton potential, as can be seen in figure 4.2). This different behaviour can be traced back to the Kähler potential, which depends on the radial position (see e.g. (B.23) and $(\overline{B .24})$ ) but not on the angular position at the tip (see (5.4)).

\section{C.2 Radial stability}

In this appendix we analyze the radial stability for a D3-brane at the tip of a deformed conifold. We want to find the condition to satisfy

$$
\left.\frac{\partial}{\partial r} V(z)\right|_{r=\varepsilon^{2 / 3}}>0
$$

The potential (5.10), as we already discussed, is valid only at the tip. Actually, in (5.6) there are some other terms, coming from the off-diagonal elements of the Kähler metric (5.6) or the additional term in $K^{\overline{1} 1}$, that are zero at the tip, but whose $r$ derivative might be non-vanishing. The additional term in $K^{\overline{1} 1}$ has two factors, $k_{\bar{l}}$ and $k_{h}$, that vanish at the tip, therefore also the $r$ derivative vanishes there. Only the off diagonal elements 
of the Kähler metric (5.6) contributes and we call these additional terms $V_{\text {off }}$ for "off diagonal".

The scalar potential depends on the radial position of the D3-brane $r$ via the Kähler potential in (4.11) and via the non-perturbative superpotential $W_{\mathrm{np}}$. We decompose $\partial_{r} V$ in four terms, the first three coming from (5.10) and the last one from $V_{\text {off }}$ :

1. All terms in 5.10 coming from the $r$ dependence in $U(r)$. Using the explicit metric of the deformed conifold near the tip we have

$$
\begin{aligned}
U & =2 \tau-\gamma k(z, \bar{z}) \\
& \simeq 2 \tau-\gamma \frac{c}{\varepsilon^{2 / 3}}\left(k_{0}+\sum_{A=1}^{4}\left|z_{A}\right|^{2}\right)=2 \tau-\gamma \frac{c}{\varepsilon^{2 / 3}}\left(k_{0}+r^{3}\right) .
\end{aligned}
$$

We will indicate this contribution as

$$
V_{r 1} \equiv \frac{\partial U}{\partial r} \frac{\partial}{\partial U}\left(V_{u p}+V_{K K L T}+\Delta V\right)
$$

2. All terms in (5.10) coming from $\partial_{r} \Delta V$ where we neglect the terms coming form $\partial_{r} U(r)$ that appear already in $V_{r 1}$. We will indicate this contribution as

$$
V_{r 2} \equiv \frac{\partial}{\partial r} \Delta V\left(U=2 \tau-\gamma k_{0}\right)
$$

3. All the remaining terms from (5.10), i.e. those coming from $\partial_{r} A(z)$ in $V_{K K L T}$. We will indicate this contribution as

$$
V_{r 3} \equiv \frac{\partial A(z)}{\partial r} \frac{\partial}{\partial A(z)} V_{K K L T}
$$

4. Finally the term $\partial_{r} V_{\text {off }}$ evaluated at the tip. It comes from the off-diagonal terms

$$
e^{\kappa_{4}^{2} K}\left(K^{\bar{T} i} \overline{D_{T} W} D_{i} W+\text { c.c. }\right) \text {. }
$$

To get a non vanishing contribution the $r$ derivative has to hit the $k_{l}$ in $K^{\bar{T} i}$, therefore

$$
V_{r 4} \equiv 2 \frac{\kappa_{4}^{2}}{3 U^{2}} W_{i} \overline{D_{T} W} k^{\overline{l i}} \frac{\partial}{\partial r} k_{\bar{l}}
$$

Let us start with $V_{r 1}$. Using (5.13), (5.14) and (5.17) it is straightforward to obtain a long expression for $V_{r 1}$. To estimate it, we use the parameterization $(\mathrm{C.2})$ and $(\mathrm{C} .1)$ for $D$ and $W_{0}$. Then we substitute the minimum for the volume estimated in (C.4), i.e. $\tau \simeq \tau_{u p}$. Finally we look at the leading terms in the $\varepsilon / \mu$ and large volume expansion; the result is

$$
V_{r 1} \simeq \frac{\kappa_{4}^{2}\left|A_{0}\right|^{2} a^{2} \gamma c e^{-2 a \tau_{0}} \varepsilon^{2 / 3}}{4 \tau_{0}^{2}}\left[(b \beta-3)+\frac{b \beta}{a \tau_{0}}+\frac{\varepsilon^{4 / 3}}{c n^{2} \mu^{2} a^{2} \tau_{0}}+\ldots\right]
$$


To calculate $V_{r 2}$, we substitute in (5.14) the embedding (5.12) and $U=2 \tau-\gamma k_{0}$. Then using the chain rule (and the fact that for constant $U, \partial_{x_{2}} \Delta V=\partial_{x_{3}} \Delta V=\partial_{x_{4}} \Delta V=0$ ) we can write

$$
V_{r 2}=\frac{\partial x_{1}}{\partial r} \frac{\partial}{\partial x_{1}} \Delta V \simeq \frac{3}{4} \varepsilon^{1 / 3} E \frac{\partial}{\partial x_{1}} \Delta V
$$

where we used the result A.20 of appendix A.2 remember that $-1<E\left(\phi_{1}, \theta_{1}, \psi\right)<1$. Following the same steps as for $V_{r 1}$, we can estimate $\Delta V_{x_{1}}$; the result is

$$
V_{r 2} \simeq-\frac{\left|A_{0}\right|^{2} e^{-2 a \tau_{0}} E \cos \phi}{8 \mu^{2} n^{2} \gamma c \tau_{0}^{2}}+\ldots
$$

For $V_{r 3}$ we obtain

$$
\begin{aligned}
V_{r 3} & =\frac{\partial x_{1}}{\partial r} \frac{\partial A\left(x_{1}\right)}{\partial x_{1}} \frac{\partial}{\partial A\left(x_{1}\right)} V_{K K L T} \\
& =\frac{|A|^{2} a e^{-2 a \tau_{0}} \varepsilon^{1 / 3}}{8 \mu n \tau_{0}^{2}} E\left[(b \beta-3)+\frac{b \beta(14-3 b \beta)}{4 a \tau_{0}}+\ldots\right] .
\end{aligned}
$$

Finally we come to $V_{r 4}$. Using again the chain rule (it is not necessary to distinguish $\partial_{z_{i}}$ from $\partial_{\bar{z}_{i}}$ because they appear symmetrically in $k(z, \bar{z})$ and at the tip they are both equal to $x_{i}$ ) we obtain

$$
\begin{aligned}
V_{r 4} & =2 \frac{\kappa_{4}^{2} \overline{D_{T} W}}{3 U^{2}} W_{i} k^{\overline{l i}} \frac{\partial z_{j}}{\partial r} \frac{\partial}{\partial z_{j}} k_{\bar{l}}=2 \frac{\kappa_{4}^{2} \overline{D_{T} W}}{3 U^{2}} \frac{\partial z_{j}}{\partial r} \delta_{i j} W_{j} \\
& =2 \frac{\kappa_{4}^{2} \overline{D_{T} W}}{3 U^{2}} \frac{\partial z_{1}}{\partial r} \frac{A\left(x_{1}\right) e^{-a \tau}}{n\left(\mu-x_{1}\right)} \\
& \simeq \frac{2}{3} 4 E \varepsilon^{1 / 3} \frac{A_{0} e^{-a \tau_{0}}}{n \mu} \frac{\beta b A_{0} e^{-a \tau_{0}}}{3\left(2 \tau_{0}\right)^{3}}=\frac{|A|^{2} a e^{-a \tau_{0}} \varepsilon^{1 / 3}}{8 \mu n \tau_{0}^{2}} E \frac{b \beta}{2 a \tau_{0}},
\end{aligned}
$$

from which it is transparent that $V_{r 4}$ is of the same order as the subleading term of $V_{r 3}$ in C.15).

We want now to compare these four contributions to understand which is the leading one and in which regime. An interesting case to consider is $b=3$, because explicit uplifting mechanisms exist with this scaling. We are not aware of any explicit model with $b>3$ but it would be intersting to investigate this possibility, e.g. in the framework of Fterm uplifting. Other consideration about the role of the uplifting term can be found in appendix 4.7. As we argued in the main text, we will be intersted in $\beta \simeq 1$ such that the cosmological constant after inflation is negligibly small. Then a subtlety arises when $b=3$ and $\beta \simeq 1$, because the leading term of both $V_{r 1}$ and $V_{r 3}$ receives and additional suppression by the coefficient $(b \beta-3)$, which is not taken into account by the $\varepsilon / \mu$ and large volume expansion. We need therefore to estimate $(\beta-1)$. 


\section{C.2.1 Radial stability in the $C \ll B$ regime (DBI inflation)}

Consider the expression for $\Lambda$ in (5.23). If we want the cosmological constant to be negligibly small after inflation, then we need $(\beta-1)$ to be of order $\left(a \tau_{0}\right)^{-2}$, so that the leading term cancel the next to leading one. This tells us that in the case $b=3$, the $(b \beta-3)$ term in $V_{r 1}$ and $V_{r 3}$ becomes subleading with respect to the next to leading one which is suppressed by just a relative $\left(a \tau_{0}\right)^{-1}$ factor. We are now ready to compare the three contributions:

$$
\begin{aligned}
& V_{r 1} \gg V_{r 3}, V_{r 4} \Longleftrightarrow(2 \pi \gamma c \mu) \varepsilon^{1 / 3} \gg 1 \\
& V_{r 1} \gg V_{r 2} \Longleftrightarrow\left\{\begin{array}{lll}
(2 \pi \gamma c \mu) \varepsilon^{1 / 3} \gg 1 & \text { for } & b \neq 3 \\
(2 \pi \gamma c \mu) \varepsilon^{1 / 3} \gg \sqrt{a \tau_{0}} & \text { for } & b=3
\end{array}\right.
\end{aligned}
$$

where we have substituted an $=2 \pi$.

We remind the reader that in section 5.4 .1 we obtained the conditions

$$
C \ll B \Longleftrightarrow\left\{\begin{array}{lll}
(2 \pi \gamma c \mu) \varepsilon^{1 / 3} \gg 1 & \text { for } \quad b \neq 3 \\
(2 \pi \gamma c \mu) \varepsilon^{1 / 3} \gg \frac{4}{15} a \tau_{0} & \text { for } \quad b=3
\end{array}\right.
$$

It follows that in the regime $C \ll B$, which is the one we consider in section 5.5.1 and 5.5.3, $V_{r 1}$ is always the leading contribution. From C.12 we see that the derivative in the radial direction is positive, i.e. a D3-brane at the tip will not move in the radial direction, as long as $b \geq 3$.

\section{C.2.2 Radial stability in the $2 C=B$ regime (slow-roll hilltop inflation)}

The regime $C=B / 2$ requires a separate discussion. As we saw in section 5.5.2, with this fine tuning, slow-roll hilltop inflation can take place.

Let us start with the case $b=3$. As discussed in section 5.5.1, to allow for slow-roll inflation at the top of the potential we need to fine tune the parameters such that $2 C=$ $B=\Lambda$, which from (C.14 and C.15 implies

$$
2 C=B \quad \Longleftrightarrow \quad(2 \pi \mu c \gamma) \varepsilon^{1 / 3}=\frac{4}{15} a \tau_{0} .
$$

Using this new constraint the condition (C.16) for the radial stability becomes:

$$
0<V_{r 1}+V_{r 2}+V_{r 3}+V_{r 4}=\frac{|A|^{2} e^{-2 a \tau_{0}}}{40 \mu^{2} c n^{2} \gamma \tau_{0}^{2}}\left[\frac{32 a \tau_{0}}{15}+E(7-5 \cos \phi)\right] .
$$

Using the fact that $|E|<1$, one obtains that the above inequality is satisfied for $a \tau_{0}>5.6$ and an arbitrary value of the angular coordinates. To neglect higher instanton contributions to the non-perturbative superpotential we have to require $a \tau_{c r} \sim a \tau_{0} \gg 1$. Therefore, 
we conclude that in the case $2 C=B$ and $b=3$ the D3-brane at the tip is (meta)stable in the radial direction.

Let us now consider the case $b>3$, then

$$
B=2 C \quad \Longleftrightarrow \quad(2 \pi c \mu \gamma) \varepsilon^{1 / 3}=1
$$

Using this constraint and taking for concreteness $b=4$, the condition (C.16) for the radial stability becomes:

$$
0<V_{r 1}+V_{r 2}+V_{r 3}+V_{r 4}=\frac{1}{8 \mu^{2} c n^{2} \gamma \tau_{0}^{2}}[2+E(1-\cos \phi)] .
$$

The inequality is fulfilled by any value of the angular coordinates, except for the case $E=-1$ and $\phi=\pi$ which saturates it. Anyway in the case we are considering $B=2 C$, (slow-roll) inflation takes place next to the top of the potential, where $(1-\cos \phi)$ is very small and therefore the inequality is confortably satisfied.

\section{C.3 From one to many}

In this appendix we obtain the Kähler potential and the non-perturbative superpotential when $N_{D 3}$ D3-branes are present.

\section{C.3.1 Kähler potential}

It was proposed in [80] that in presence of a D3-brane, the Kähler potential should be modified to

$$
\kappa_{4}^{2} K=-3 \log [T+\bar{T}-\gamma k(z, \bar{z})]
$$

where $\gamma$ is a the constant describing the strength of gravitational backreaction, essentially proportional to $G_{N}$ (Newton constant) times the tension of the D3-brane $T_{3}$ and $k(z, \bar{z})$ is the Kähler potential of the Calabi Yau evaluated at the position $z$ of the D3-brane. Two arguments were given to support this proposal. The first is based on the observation that the D3-brane in the presence of three form fluxes is BPS and does not feel any force. In addition, its inclusion should not spoil the no-scale structure. This leads to the natural guess C.20 with an arbitrary function $k(z, \bar{z})$, for which one can easily check that the F-term potential vanishes, leaving $T$ and $z$ as flat directions.

The second argument consists in noticing that the kinetic term deduced from the supergravity approximation should reproduce, at leading order, the DBI action for the D3-brane. The 10-dimensional metric can be written as

$$
d s^{2}=h^{-1 / 2}(Y) e^{-6 u} g_{\mu \nu} d x^{\mu} d x^{\nu}+h^{1 / 2}(Y) e^{2 u} \tilde{g}_{i j} d Y^{i} d Y^{j},
$$


so that we have singled out the breathing mode of the compact manifold $e^{2 u}$ with respect to a fiducial metric $\tilde{g}_{i j}$. The expansion of the DBI action for small velocities gives

$$
S_{\mathrm{DBI}} \simeq-T_{3} \int d^{4} x \sqrt{g} e^{-4 u} \partial_{\mu} z^{i} \partial^{\mu} z^{j} \tilde{g}_{i j}
$$

To obtain this action from the second derivative of the Kähler potential as supergravity dictates, one is led to the identification of the breathing mode with the Kähler volume modulus plus the $X$ dependent shift [80, 65]:

$$
e^{4 u}=T+\bar{T}-\left(\frac{T_{3}}{3 M_{P l}^{2}}\right) k(z, \bar{z}) .
$$

To generalize to the case of $N_{D 3}$ D3-branes it seems natural to use the same two criteria as above. A natural and simple guess is therefore

$$
\kappa_{4}^{2} K=-3 \log \left[T+\bar{T}-\left(\frac{T_{3}}{3 M_{P l}^{2}}\right) \sum_{s=1}^{N_{D 3}} k\left(z_{s}, \bar{z}_{s}\right)\right],
$$

where the index $s$ enumerates the D3-branes, located at $z_{s}$. This Kähler potential again respects the no-scale structure. Also, after neglecting terms of order $e^{-8 u}$, it gives a kinetic term that is the sum of $N_{D 3}$ independent copies of the action (C.22); this is what we expect, given that the D3-branes are mutually BPS. This property is important for the assisted inflation mechanism that requires the various inflatons to be weakly interacting.

\section{C.3.2 Superpotential}

In [69] the dependence of the non-perturbative superpotential on the D3-brane position was calculated in the background of a warped throat. In the case of an E3-instanton or a stack of D7-branes with gaugino condensation, wrapping a divisor $\Sigma$ of the Calabi Yau, this superpotential is proportional to

$$
W_{\mathrm{np}} \propto e^{-T_{3} V_{\Sigma}^{w} / n}
$$

where $V_{\Sigma}^{w}$ is the warped volume of the divisor $\Sigma$ (the label $w$ is to remind that the full metric, including the warp factor has to be use) ; $n$ is 1 for E3-brane and $N_{D 7}$ for gaugino condensation on a stack of $N_{D 7}$ branes. As in the main text, we indicate with $g$ the holomorphic function whose zeros define the divisor $\Sigma$.

It was argued in [76] that $W_{\mathrm{np}}$ has to depend on the D3-brane moduli. The argument consists in noticing that $W_{\mathrm{np}}(z, \bar{z})$ is the product of two factors, respectively a section of the line bundle and inverse line bundle defined by the divisor $\Sigma$. Then a theorem guarantees that it has to vanish on (a surface homotopic to) the divisor $\Sigma$. 
The explicit dependence was first calculated in [70] for some toroidal orientifolds via a one loop open string calculation. In [69] the tree level closed string dual was considered. In the following we review the method of [69] and generalize the result to the case of $N_{D 3}$ D3-branes. The idea is that a D3-brane backreacts on the metric, in particular changing the warp factor. This in turn changes the volume $V_{\Sigma}^{w}$ in (C.25). Schematically

$$
h(Y)=h_{0}(Y)+\delta h(Y, z)
$$

where $Y$ is the point where the warping is evaluated, and $z$ is the D3-brane position. Integrating over the divisor $\Sigma$ we obtain

$$
\begin{aligned}
V_{\Sigma}^{w} & =\left(V_{\Sigma}^{w}\right)_{0}+\delta V_{\Sigma}^{w}(X) \\
& =\int_{\Sigma} d^{4} Y \sqrt{g^{i n d}(z, Y)}\left[h_{0}(Y)+\delta h(z ; Y)\right]=\tilde{V}_{\Sigma} h_{0}(T+\bar{T})+\Re \xi(z),
\end{aligned}
$$

where we have made explicit that the corrected volume is still the real part of a holomorphic function. $V_{\Sigma}^{w}$ is in fact proportional to the real part of the gauge kinetic function that supersymmetry dictates to be holomorphic. Finally

$$
\begin{aligned}
W_{\mathrm{np}} & \propto \exp \left(-\frac{T_{3} \xi(X)}{n}\right) \exp \left(-\frac{T_{3} V_{\Sigma} h_{0} T}{n}\right) \\
& \equiv A(X) e^{-a T} .
\end{aligned}
$$

The advantage of this closed string approach is that $\delta h$ is directly calculated from the Laplace equation

$$
-\nabla_{Y}^{2} \delta h(X, Y)=\kappa_{10} \rho_{m},
$$

using the Green's function method. Here $\rho_{m}$ is the all inclusive energy density.

Solving the Laplace equation for $\delta h$ and integrating over a divisor defined by the zeros of the holomorphic function $g$, one obtains the dependence of the superpotential on the position $X$ of a single D3-branes [69]

$$
W_{\mathrm{np}}=A(z) e^{-a T}=A_{0} g(z)^{1 / n} e^{-a T},
$$

where $A_{0}$ might depend on the dilaton and the complex structure moduli. To generalize to $N_{D 3}$ D3-branes at positions $z_{s}$ one has to solve the Laplace equation

$$
\begin{aligned}
-\nabla_{Y}^{2} \delta h\left(z_{s} ; Y\right) & =2 T_{3} \kappa_{10}^{2}\left[\sum_{s=1}^{N_{D 3}} \frac{\delta^{(6)}\left(z_{s}-Y\right)}{\sqrt{g(Y)}}-\rho_{b g}(Y)\right] \\
& =2 T_{3} \kappa_{10}^{2}\left[\sum_{s=1}^{N_{D 3}} \frac{\delta^{(6)}\left(z_{s}-Y\right)}{\sqrt{g(Y)}}-N \frac{\delta\left(z_{0}-Y\right)}{\sqrt{g(Y)}}\right],
\end{aligned}
$$

where $z_{0}$ is a reference point (for example the tip of the cone). With this specific choice of $\rho_{b g}$ we get rid of a logarithmic divergence in $\delta V_{\Sigma}^{w}$. This leads to an interpretation of 
$\delta h(X ; Y)$ as the variation of the warp factor $h$ when the D3-branes are moved from $X_{0}$ to $X_{s}$.

Given the linearity of the Laplace equation the solution for (C.31) is just a sum over $X_{s}$ of the solution of the single D3-brane problem. Once $\delta h(X ; Y)$ is integrated over the divisor $\Sigma$ and exponentiated, we obtain the superpotential

$$
W_{\mathrm{np}}=A(X) e^{-a T}=A_{0} \prod_{s=1}^{N_{D 3}} f\left(X_{s}\right)^{1 / n} e^{-a T} .
$$

We notice that the superpotential C.32 reproduces what we would expect from Ganor's argument [76]: $W_{\mathrm{np}}$ vanishes if any of the D3-branes hits the divisor $\Sigma$. 


\section{Technical details on soft terms from LVS}

In the following series of appendices, we have collected several calculations whose results have been used in chapter 6. We start in appendix D.1.2 and D.1.1 where we give some details about the LVS for $\mathbb{P}_{[1,1,1,6,9]}^{4}$ and the generalization to many Kähler moduli; in appendix D.2 we calculate the inverse Kähler metric for the loop corrected Kähler potential (6.25); in appendix D.3 we start reviewing the no-scale structure both in type IIA and IIB and continue considering corrections that break this structure; in appendix D.4 we discuss how fluxes would change the discussion in chapter 6, in appendix D.5 we review the orientifold calculation of the loop correction performed in [171]; finally in appendix D.6 we discuss the factorized approximation.

\section{D.1 Some details on LVS}

In this appendix we collect some details on the minimization of the potential in LVS, mainly reviewing the results of [169, 146], but filling in some details. The minimization with respect to the axions (i.e. the imaginary parts of the Kähler moduli) is performed for an arbitrary number of Kähler moduli, while for the minimization of the real parts, we restrict to the example of the hypersurface in $\mathbb{P}_{[1,1,1,6,9]}^{4}$ discussed throughout the main text.

\section{D.1.1 LVS for $\mathbb{P}_{[1,1,1,6,9]}^{4}$}

Here we give some more numerical details on large-volume stabilization in the $\mathbb{P}_{[1,1,1,6,9}^{4}$ orientifold. The relevant features of this Calabi-Yau have been described in section 6.2.3. The leading terms of the scalar potential are

$$
V e^{-K_{\mathrm{cs}}}=\frac{\lambda \sqrt{\tau} e^{-2 a \tau}}{\mathcal{V}}-\frac{\mu}{\mathcal{V}^{2}} \tau e^{-a \tau}+\frac{\nu}{\mathcal{V}^{3}}
$$

where we use $\tau=\tau_{s}$ and $\mathcal{V}$ as the independent variables and for the expansion we have in mind the limit (6.15). The minimum of this potential under the assumption that $a \tau \gg 1$ 
is given by

$$
\begin{aligned}
\tau & =\left(\frac{4 \nu \lambda}{\mu^{2}}\right)^{2 / 3} \\
\mathcal{V} & =\frac{\mu}{2 \lambda}\left(\frac{4 \nu \lambda}{\mu^{2}}\right)^{1 / 3} e^{a \tau} .
\end{aligned}
$$

In the $\mathbb{P}_{[1,1,1,6,9]}^{4}$ orientifold the coefficients $\lambda, \mu$ and $\nu$ can be calculated explicitly, yielding

$$
\lambda=\frac{12 \sqrt{2} a^{2}|A|^{2}}{S_{1}}, \quad \mu=\frac{2 a\left|A W_{0}\right|}{S_{1}} \text { and } \nu=\xi \frac{3}{8} \sqrt{S_{1}}\left|W_{0}\right|^{2}
$$

We notice that the value of $\tau$ at the minimum is determined only by the Euler number $\tau \propto \chi^{2 / 3}$ and the value of the dilaton $S_{1}$ at its minimum. An example of a set of possible parameters (using $a=2 \pi / 10, A=1, S_{1}=10$ and $W_{0}=10$ ) is

$$
\begin{array}{rlrl}
\xi=-\frac{\zeta(3) \chi}{2(2 \pi)^{3}} & \simeq 1.31 \quad \longrightarrow \quad \nu & \simeq 155, \\
\lambda & \simeq 0.67, & \mu & =\frac{4 \pi}{10} .
\end{array}
$$

There is an unknown overall factor $e^{K_{c s}}$ that does not change the shape of the potential and so leaves the position of the minima unchanged. For the parameters given in equation (D.4), the minimum is at $\tau \simeq 41.1$ and $\mathcal{V} \simeq 9.96 \cdot 10^{11}$. These values come from equation (D.2) which is just approximated using the assumption $a \tau \gg 1$. This solution has the shortcoming that, if one is interested in the value of the potential at the minimum, after substitution of (D.2) into (D.1), one finds $V=0$. If instead one solves the exact equation for the minimum of the potential numerically, the result is $V \simeq-6.6 \cdot 10^{-37}$ at the point $\tau \simeq 41.7$ and $\mathcal{V} \simeq 1.3810^{12}$. From this one checks that, apart from the shortcoming that $V=0$, the approximate solution gives the position of the minimum with a good precision.

\section{D.1.2 Many Kähler moduli}

The simple picture of $\mathbb{P}_{[1,1,1,6,9]}^{4}$, gets slightly more involved in models with more than two Kähler moduli, but some general statements can still be made. For a single small Kähler modulus, among the leading contributions to the potential only the one from $V_{\text {np2 }}$ is axion dependent, while the leading terms in $V_{3}$ and $V_{\mathrm{np} 1}$ are axion independent. For several small Kähler moduli, all three terms are axion dependent. However, the argument that the leading term in $V_{\mathrm{np} 2}$ only receives a sign change due to axion stabilization generalizes (and holds also for the regular KKLT scenario with relatively small volume, see e.g. [146], section 3.2). 
Indeed, with the superpotential (6.11) one obtains

$$
\begin{aligned}
& V_{\mathrm{np} 1}=e^{K} G^{\bar{j} i}\left[a_{i} a_{j}\left|A_{i} A_{j}\right| e^{-a_{i} \tau_{i}-a_{j} \tau_{j}} \cos \left(-a_{i} b_{i}+a_{j} b_{j}+\beta_{i}-\beta_{j}\right)\right], \\
& V_{\mathrm{np} 2}=-2 e^{K} a_{i} G^{\bar{k} i} K_{\bar{k}}\left[\left|A_{i} W_{0}\right| e^{-a_{i} \tau_{i}} \cos \left(-a_{i} b_{i}+\beta_{i}-\beta_{W_{0}}\right)\right. \\
& \left.+\left|A_{i} A_{j}\right| e^{-a_{i} \tau_{i}-a_{j} \tau_{j}} \cos \left(-a_{i} b_{i}+a_{j} b_{j}+\beta_{i}-\beta_{j}\right)\right], \\
& V_{3}=e^{K}\left(G^{\bar{k} l} K_{\bar{k}} K_{l}-3\right)\left[\left|W_{0}\right|^{2}+2\left|W_{0} A_{i}\right| e^{-a_{i} \tau_{i}} \cos \left(-a_{i} b_{i}+\beta_{i}-\beta_{W_{0}}\right)\right. \\
& \left.+\left|A_{i} A_{j}\right| e^{-a_{i} \tau_{i}-a_{j} \tau_{j}} \cos \left(-a_{i} b_{i}+a_{j} b_{j}+\beta_{i}-\beta_{j}\right)\right],
\end{aligned}
$$

where $A_{i}=\left|A_{i}\right| e^{i \beta_{i}}, W_{0}=\left|W_{0}\right| e^{i \beta_{W_{0}}}$ and a sum over repeated indices is understood throughout. As the only dependence on the axions is in form of cosines, one can easily see that this potential has a minimum for

$$
a_{i} b_{i}=-\beta_{W_{0}}+\beta_{i}+n_{i} \pi \quad, \quad n_{i} \in 2 \mathbb{Z}+1 .
$$

We notice that the minimum of the $b_{i}$ depends on the (already fixed) complex structure moduli, but it is independent of the Kähler moduli.

In the regime (6.15) the scalar potential again contains three terms at leading order,

$$
\begin{aligned}
V_{\mathrm{np} 1} & \sim 2 e^{K_{\mathrm{cs}}} \frac{a_{i} a_{j}\left|A_{i} A_{j}\right| e^{-a_{i} \tau_{i}-a_{j} \tau_{j}} M_{j}^{l} M_{i}^{k}\left(-\mathcal{V} \mathcal{V}_{l k}+\mathcal{V}_{l} \mathcal{V}_{k}\right)}{S_{1} \mathcal{V}^{2}}+\ldots, \\
V_{\mathrm{np} 2} & \sim-2 e^{K_{\mathrm{cs}}} \frac{a_{i}\left|A_{i} W_{0}\right| e^{-a_{i} \tau_{i}} \tau_{i}}{S_{1} \mathcal{V}^{2}}+\ldots, \\
V_{3} & \sim e^{K_{\mathrm{cs}}} \frac{3 \xi S_{1}^{1 / 2}}{8 \mathcal{V}^{3}}\left|W_{0}\right|^{2}+\ldots,
\end{aligned}
$$

where the sum over $i$ and $j$ effectively only picks up terms from the small moduli because of the exponential suppression of $V_{\mathrm{np} 1}$ and $V_{\mathrm{np} 2}$. Moreover, for $V_{\mathrm{np} 1}$ we used the form (D.34) for the inverse of the moduli metric with respect to the basis (D.28). The Kähler moduli appearing in the nonperturbative superpotential are linear combinations of these, which we account for by a basis-changing matrix $M_{i}^{k}$, i.e.

$$
T_{i}=M_{i}^{k} \tilde{T}_{k},
$$

where $\tilde{T}_{k}$ are the fields defined in $(\mathrm{D} .28)$ and $T_{i}$ are the Kähler moduli appearing in the nonperturbative superpotential. (Another way of saying this is that the real parts of $T_{i}$ measure the volumes of a basis of divisors that have the right properties to contribute to the nonperturbative superpotential.) In the second term we used

$$
G^{\bar{k} i} K_{\bar{k}}=-2 \tau_{i}+\ldots=-2 \operatorname{Re} T_{i}+\ldots .
$$


In the basis (D.28), this would follow straightforwardly from (D.32), (D.34) and the relations (D.25), but it holds equally well after a change of basis, because both sides of (D.9) transform linearly under a change of basis (D.8).

Note finally that the ellipsis in (D.7) and (D.9) stand for subleading corrections in the large volume limit (assuming also (6.15)).

\section{D.2 Loop corrected inverse Kähler metric for $\mathbb{P}_{[1,1,1,6,9]}^{4}$}

We now have a closer look at the inverse metric from the Kähler potential in equation (6.25). We invert the $4 \times 4$ matrix and focus on the four terms that appear in the scalar potential for the Kähler moduli,

$$
\begin{aligned}
G^{\bar{b} b} & =\frac{4}{3} \tau_{b}^{2}+\mathcal{O}\left(\tau_{b}\right) \\
G^{\bar{b} s}=G^{\bar{s} b} & =4 \tau_{b} \tau_{s}\left(1+\frac{6 \mathcal{E}_{s}^{K}}{\Delta}\right)+\mathcal{O}\left(\tau_{b}^{0}\right), \\
G^{\bar{s} s} & =\frac{8}{3} \tau_{b}^{3 / 2} \sqrt{\tau_{s}} \frac{\sqrt{2} S_{1} \tau_{s}}{\Delta}+\mathcal{O}\left(\tau_{b}^{1 / 2}\right),
\end{aligned}
$$

where we have performed an expansion in $\tau_{b} \simeq \mathcal{V}^{2 / 3}$ and the quantity $\Delta$ was introduced in 6.29 . We notice that only $G^{\bar{b} b}$ is not corrected at leading order. The apparent divergence from zeros of the denominator $\Delta$ is an artifact of the expansion. In fact, the determinant of the (entire) Kähler metric behaves as

$$
\operatorname{det} G \sim A \tau_{b}^{-7 / 2}+B \tau_{b}^{-9 / 2}+\ldots
$$

for some expressions $s^{1} A$ and $B$, which depend on the moduli $\tau_{s}, u$ and $S_{1}$. In particular, one finds $A \sim \Delta$, but $B$ does not vanish at a zero of $\Delta$. Thus, in general the expansion in large $\tau_{b}$ picks up the factor $A$, which is responsible for the apparent divergence in (D.10). However, this is fictitious because when $\Delta=0$, the next term proportional to $B$ is nonvanishing and the determinant stays away from zero. Indeed, we do not expect to find any zero of the determinant in the range of validity of the parameters.

If $\mathcal{E}_{s}^{K} \ll\left(S_{1} \tau_{s}\right)$, one can further expand D.10 with respect to $\mathcal{E}_{s}^{K} /\left(S_{1} \tau_{s}\right)$, yielding

$$
\begin{aligned}
G^{\bar{b} b} & =\frac{4}{3} \tau_{b}^{2}+\mathcal{O}\left(\tau_{b}\right) \\
G^{\bar{b} s}=G^{\bar{s} b} & =4 \tau_{b} \tau_{s}\left(1+\frac{3 \sqrt{2} \mathcal{E}_{s}^{K}}{S_{1} \tau_{s}}+\mathcal{O}\left(\frac{\left(\mathcal{E}_{s}^{K}\right)^{2}}{S_{1}^{2} \tau_{s}^{2}}\right)\right) \\
G^{\bar{s} s} & =\frac{8}{3} \tau_{b}^{3 / 2} \sqrt{\tau_{s}}\left(1+\frac{3 \mathcal{E}_{s}^{K}}{\sqrt{2} S_{1} \tau_{s}}+\mathcal{O}\left(\frac{\left(\mathcal{E}_{s}^{K}\right)^{2}}{S_{1}^{2} \tau_{s}^{2}}\right)\right)
\end{aligned}
$$

\footnotetext{
${ }^{1}$ This $A$ has nothing to do with the $A$ in $W_{\text {np }}$.
} 
Depending on the values of the moduli $\left(\tau_{s}\right.$ and $\left.S_{1}\right)$, this expansion may or may not be useful. In general, only the expansion in $\tau_{b}$ makes sense and one has to deal with the full expressions (D.10). That is what we did in section 6.3.3.

\section{D.3 No-scale Kähler potential in type II string theory}

In this appendix we review why compactification of type IIA and type IIB theory on general Calabi-Yau manifolds, or orientifolds thereof, lead to no-scale (F-term) potentials if

i) the superpotential does not depend on the Kähler moduli

and if

ii) one uses the tree-level form of the Kähler potential.

(Of course, in LVS neither $i$ ) nor $i i$ ) holds, but one can think of jointly imposing $i$ ) and ii) as a zeroth-order approximation, that we will successively move away from in later subsections of this appendix.)

If the moduli spaces of Kähler and complex structure moduli factorize (see appendix D.6 for more details on this), and under assumption $i$ ), the F-term potential takes the form

$$
\begin{aligned}
V & =e^{K}\left(G^{\bar{I} J} D_{\bar{I}} \bar{W} D_{J} W-3|W|^{2}\right) \\
& =e^{K}\left(G^{\bar{a} b} D_{\bar{a}} \bar{W} D_{b} W+\left(G^{\bar{\imath} j} K_{\bar{\imath}} K_{j}-3\right)|W|^{2}\right) .
\end{aligned}
$$

The indices $a$ and $b$ run over the complex structure moduli and the dilaton, $i, j$ over the Kähler moduli and $I$ and $J$ refer to all moduli.

The condition for a no-scale potential ( $V=0$ for the Kähler moduli) is then

$$
G^{\bar{\imath} j} K_{\bar{\imath}} K_{j}=3
$$

and we will verify in turn that this is fulfilled in both type IIA and type IIB Calabi-Yau compactifications, if one uses the tree-level Kähler potential, as in assumption ii). In that case, the moduli spaces of Kähler and complex structure moduli do factorize exactly.

\section{D.3.1 No-scale structure in type IIA}

The tree level Kähler potential for the Kähler moduli is

$$
K=-\ln \left[\frac{1}{48} d_{i j k}(\sigma+\bar{\sigma})_{i}(\sigma+\bar{\sigma})_{j}(\sigma+\bar{\sigma})_{k}\right]=-\ln \left[\frac{1}{6} d_{i j k} t_{i} t_{j} t_{k}\right]=-\ln (\mathcal{V})
$$


where $d_{i j k}$ are the intersection numbers of the Calabi-Yau,

$$
d_{i j k}=\int_{C Y} \omega_{i} \wedge \omega_{j} \wedge \omega_{k}
$$

and

$$
\sigma_{i}=t_{i}+i c_{i}
$$

are the complexified Kähler moduli whose real parts $t_{i}$ represent the volumes of 2-cycles and whose imaginary parts originate from the expansion of the NSNS 2-form. Using the Kähler form

$$
J=t_{i} \omega_{i}
$$

of the Calabi-Yau, it is useful to introduce the notation

$$
\begin{aligned}
\mathcal{V} & =\frac{1}{6} \int J \wedge J \wedge J=\frac{1}{6} d_{i j k} t_{i} t_{j} t_{k}, \\
\mathcal{V}_{i} & =\frac{1}{2} \int \omega_{i} \wedge J \wedge J=\frac{1}{2} d_{i j k} t_{j} t_{k}, \\
\mathcal{V}_{i j} & =\int \omega_{i} \wedge \omega_{j} \wedge J=d_{i j k} t_{k} .
\end{aligned}
$$

Note that here the index $i$ does not denote a derivative with respect to the Kähler variables (in contrast to subscripts on the Kähler potential $K$ ). Instead, one has the relations $\mathcal{V}_{i}=2 \partial_{\sigma_{i}} \mathcal{V}$ and $\mathcal{V}_{i j}=4 \partial_{\sigma_{i}} \partial_{\bar{\sigma}_{\bar{\jmath}}} \mathcal{V}$. It is straightforward to calculate

$$
K_{i}=-\frac{\mathcal{V}_{i}}{2 \mathcal{V}}=K_{\bar{\imath}} \quad G_{i \bar{\jmath}}=K_{i \bar{\jmath}}=-\frac{1}{4}\left(\frac{\mathcal{V}_{i j}}{\mathcal{V}}-\frac{\mathcal{V}_{i} \mathcal{V}_{j}}{\mathcal{V}^{2}}\right)
$$

Then one can show that the inverse Kähler metric is

$$
G^{\bar{\jmath} i}=-4 \mathcal{V}^{j i} \mathcal{V}+2 t_{j} t_{i}
$$

To verify this, one has to use

$$
\mathcal{V}^{i j} \mathcal{V}_{j}=\frac{1}{2} t_{i}, \quad \mathcal{V}_{i j} t_{j}=2 \mathcal{V}_{i}, \quad \mathcal{V}_{i} t_{i}=3 \mathcal{V}
$$

Putting everything together, one arrives at

$$
G^{\bar{\imath} j} K_{\bar{\imath}} K_{j}=\left[-4 \mathcal{V}^{i j} \mathcal{V}+2 t_{i} t_{j}\right] \frac{1}{4} \frac{\mathcal{V}_{i}}{\mathcal{V}} \frac{\mathcal{V}_{j}}{\mathcal{V}}=3
$$

i.e. (D.17) is fulfilled under assumption (D.14). 


\section{D.3.2 No-scale structure in type IIB}

In the type IIB case, the tree-level Kähler potential for the Kähler moduli is

$$
K=-2 \ln \left[\frac{1}{6} d_{i j k} t_{i} t_{j} t_{k}\right]=-2 \ln (\mathcal{V}) .
$$

The difference to the IIA case is that, even if $K$ in (D.27) is expressed in terms of the 2 -cycle volumes $t_{i}$, the real parts of the good Kähler modul: 2 , $\tilde{T}_{i}$, are now the 4 -cycle volumes $\tilde{\tau}_{i}$ (the imaginary parts, on the other hand, arise from the $\mathrm{RR} 4$-form). The relation between them depends on the particular Calabi-Yau:

$$
\operatorname{Re} \tilde{T}_{i}=\tilde{\tau}_{i}=\frac{1}{2} d_{i j k} t_{j} t_{k}=\mathcal{V}_{i},
$$

which can not be inverted in general. In order to calculate $K_{i}=\partial_{\tilde{T}_{i}} K$ we note that

$$
\begin{aligned}
\partial_{t_{i}} & =\left(\partial_{t_{i}} \tilde{T}_{j}\right) \partial_{\tilde{T}_{j}}+\left(\partial_{t_{i}} \overline{\tilde{T}}_{\bar{\jmath}}\right) \partial_{\tilde{T}_{\bar{T}}} \\
& =\mathcal{V}_{i j}\left(\partial_{\tilde{T}_{j}}+\partial_{\tilde{T}_{\bar{\jmath}}}\right) .
\end{aligned}
$$

If acting on a function $F$ that only depends on $\tilde{T}+\tilde{T}$, as is the case for $K$, D.29) simplifies to

$$
\partial_{t_{i}} F(\tilde{T}+\overline{\tilde{T}})=2 \mathcal{V}_{i j} \partial_{\tilde{T}_{j}} F(\tilde{T}+\overline{\tilde{T}})
$$

where on the left hand side $\tilde{T}$ is understood as a function of $t$. Alternatively, one has

$$
\partial_{\tilde{T}_{i}} F(\tilde{T}+\overline{\tilde{T}})=\frac{1}{2} \mathcal{V}^{i j} \partial_{t_{j}} F(\tilde{T}+\overline{\tilde{T}})=\partial_{\tilde{\tilde{T}}_{\bar{\imath}}} F(\tilde{T}+\overline{\tilde{T}})
$$

Using this, one can calculate

$$
K_{i}=-\frac{2}{\mathcal{V}} \partial_{\tilde{T}_{i}} \mathcal{V}=-\frac{\mathcal{V}^{i j} \mathcal{V}_{j}}{\mathcal{V}}=-\frac{t_{i}}{2 \mathcal{V}}=K_{\bar{\imath}}
$$

where in the last step we used $(\mathrm{D} .25)$. In the same way one can calculate

$$
G_{i \bar{\jmath}}=\frac{1}{4}\left(-\frac{\mathcal{V}^{i j}}{\mathcal{V}}+\frac{1}{2} \frac{t_{i} t_{j}}{\mathcal{V}^{2}}\right)
$$

Using this formula one can check that the inverse Kähler metric is given by

$$
G^{\bar{i} j}=4\left(-\mathcal{V} \mathcal{V}_{i j}+\mathcal{V}_{i} \mathcal{V}_{j}\right)
$$

Putting everything together, no-scale structure holds also for type IIB:

$$
G^{\bar{\imath} j} K_{\bar{\imath}} K_{j}=\left(-\mathcal{V} \mathcal{V}_{i j}+\mathcal{V}_{i} \mathcal{V}_{j}\right) \frac{t_{i}}{\mathcal{V}} \frac{t_{j}}{\mathcal{V}}=3
$$

again under the assumption (D.14).

\footnotetext{
${ }^{2}$ Note that the Kähler moduli appearing in the non-perturbative superpotentials in the examples of [61] are related to the ones in $(\mathrm{D} .28)$ by a linear field redefinition. However, this does not play any role in verifying the no-scale structure at leading order, as $\mathrm{D} .35$ below is invariant under field redefinitions. We chose to make the distinction clear by using tildes for the Kähler moduli defined by (D.28).
} 


\section{D.3.3 Cancellation with just the volume modulus}

Now we relax assumption (D.14). For simplicity, let us first consider the Kähler potential

$$
K=-3 \ln (T+\bar{T})+\frac{\Xi}{(T+\bar{T})^{\lambda}},
$$

which corresponds to the case of a single Kähler modulus and the complex structure moduli and the dilaton are neglected. A generic quantum correction was added to the tree level term, which could be an $\alpha^{\prime}$ or a loop correction, depending on the value of $\lambda$. Focusing on $V_{3}$, i.e.

$$
\frac{V_{3}}{e^{K}|W|^{2}}=G^{\bar{\jmath} i} K_{\bar{\jmath}} K_{i}-3
$$

one calculates

$$
\begin{aligned}
\frac{V_{3}}{e^{K}|W|^{2}} & =\frac{\left(3(2 \tau)^{\lambda}+\xi \lambda\right)^{2}}{3(2 \tau)^{2 \lambda}+\Xi(2 \tau)^{\lambda} \lambda(\lambda+1)}-3 \\
& =3-3+\frac{\left(\lambda-\lambda^{2}\right) \Xi}{(2 \tau)^{\lambda}}+\frac{\Xi^{2} \lambda^{4}}{3(2 \tau)^{2 \lambda}}+\mathcal{O}\left(\frac{1}{\tau^{3 \lambda}}\right) .
\end{aligned}
$$

This simplified calculation gives an intuition of why the $\mathcal{E}_{b}^{K}$-term does not appear in $V_{3}$ of (6.28) whereas the $\alpha^{\prime}$ - and $\mathcal{E}_{s}^{K}$-terms contribute. When the exponent of the quantum correction is exactly 1 , there is a cancellation at leading order in the scalar potential (compare also the discussion in footnote 12). Note that since we focused on $V_{3}$ in this subsection, it did not matter whether assumption (D.13) holds or not.

\section{D.3.4 Cancellation with many Kähler moduli}

We would now like to see how the previous result is changed when we have an arbitrary number of moduli. We do not make any assumption on the dependence of the volume on the Kähler moduli ("Swiss cheese" or fibered manifolds are special cases). Due to its relevance for LVS, we consider a single correction to the Kähler potential which only depends on the large Kähler modulus $T_{b}$ (an example would be the $\alpha^{\prime}$-correction or the loop term proportional to $\mathcal{E}_{b}^{K}$, considering the moduli other than the Kähler moduli as fixed; this is allowed at leading order in a $\tau_{b}$-expansion, as we argue in appendix D.6. Thus, we take the Kähler potential to be of the form

$$
K=K^{(0)}+\delta K=-2 \ln (\mathcal{V})+\delta K\left(T_{b}, \bar{T}_{b}\right) \equiv-2 \ln (\mathcal{V})+\frac{\Xi_{b}}{\left(T_{b}+\bar{T}_{b}\right)^{\lambda}} .
$$

Again focusing on $V_{3}$, we obtain

$$
\frac{V_{3}}{e^{K}|W|^{2}}=G^{\bar{\jmath} i} K_{\bar{\jmath}} K_{i}-3=\left(G_{0}^{\bar{\jmath} i}+\delta G^{\bar{\jmath} i}\right)\left[K_{\bar{\jmath}}^{(0)}+\delta K_{\bar{\jmath}}\right]\left[K_{i}^{(0)}+\delta K_{i}\right]-3,
$$


where $\delta K_{i} \equiv \partial_{T_{i}} \delta K$ and $G_{0}^{\bar{j} i}$ is the inverse metric of appendix D.3.2 finally $\delta G^{\bar{j} i}$ is the modification of the inverse metric coming from considering the modified Kähler potential (D.39). Explicitly one has

$$
\begin{aligned}
G^{\bar{\jmath} i} & =\left(G_{i \bar{\jmath}}^{0}+\delta K_{i \bar{\jmath}}\right)^{-1} \simeq G_{0}^{\bar{\jmath} i}-G_{0}^{\bar{\jmath} h} \delta K_{h \bar{k}} G_{0}^{\bar{k} i}+\ldots, \\
\delta K_{i} & =-\frac{\lambda \Xi_{b}}{\left(2 \tau_{b}\right)^{\lambda+1}} \delta_{i b}, \quad \delta K_{i \bar{\jmath}}=\frac{\left(\lambda^{2}+\lambda\right) \Xi_{b}}{\left(2 \tau_{b}\right)^{\lambda+2}} \delta_{i b} \delta_{\bar{\jmath} b} .
\end{aligned}
$$

We now put everything together and use the results of appendix D.3.2 and formula (D.9) (which, for the unperturbed metric and Kähler potential, is an exact equality) to arrive at

$$
\begin{aligned}
\frac{V_{3}}{e^{K}|W|^{2}} & =\left[G_{0}^{\bar{\jmath} i} K_{\bar{\jmath}}^{(0)} K_{i}^{(0)}-3\right]+2\left[G_{0}^{\bar{\jmath} i} K_{\bar{\jmath}}^{(0)} \delta K_{i}\right]-\left(G_{0}^{\bar{j} k} \delta K_{k \bar{h}} G_{0}^{\bar{h} i}\right) K_{\bar{\jmath}}^{(0)} K_{i}^{(0)}+\ldots \\
& =0+\frac{4 \lambda \Xi_{b}}{\left(2 \tau_{b}\right)^{\lambda+1}} \tau_{b}-\frac{4\left(\lambda^{2}+\lambda\right) \Xi_{b}}{\left(2 \tau_{b}\right)^{\lambda+2}} \tau_{b} \tau_{b}+\ldots \\
& =\frac{\left(\lambda-\lambda^{2}\right) \Xi_{b}}{\left(2 \tau_{b}\right)^{\lambda}}+\ldots
\end{aligned}
$$

We notice that the term $1 / \tau_{b}^{\lambda}$ vanishes exactly for $\lambda=1$, independently of the explicit form of the volume in terms of the Kähler moduli. In particular, the loop correction proportional to $\mathcal{E}_{b}^{K}$ experiences a cancellation at leading order in $V_{3}$ (and it is not difficult to see that the subleading order is suppressed by $\tau_{b}^{-2 \lambda}$ ). Therefore, the loop correction is subleading in the potential compared to the $\alpha^{\prime}$ correction, even though it is leading in the Kähler potential. Next, we would like to extend this analysis to the other parts of the potential, i.e. $V_{\mathrm{np} 1}$ and $V_{\mathrm{np} 2}$.

\section{D.3.5 Perturbative corrections to $V_{\mathrm{np} 1}$ and $V_{\mathrm{np} 2}$}

We now introduce the nonpertubative superpotential into the game, i.e. relax assumption (D.13), and look at the other terms of the scalar potential, $V_{\mathrm{np} 1}$ and $V_{\mathrm{np} 2}$ (see eq. (6.12)). For this, we restrict to the $\mathbb{P}_{[1,1,1,6,9]}^{4}$ model again. The contribution $V_{\mathrm{np} 1}$ is proportional to $G^{\bar{s} s} \bar{W}_{, s} W_{, s}$. From (D.10) we see that no $\mathcal{E}_{b}^{K}$ appears at leading order. This can be understood as follows. Consider the Kähler potential (D.39) where now $\mathcal{V}$ is the volume of $\mathbb{P}_{[1,1,1,6,9]}^{4}$, given in 6.14). Then the scaling with the large Kähler modulus $\tau_{b}$ is schematically given by

$$
\begin{aligned}
G^{\bar{s} s} & \simeq G_{0}^{\bar{s} s}-G_{0}^{\overline{s b}} \delta K_{b \bar{b}} G_{0}^{\bar{b} s}+\ldots \\
& \sim \tau_{b}^{3 / 2}+\tau_{b}^{2} \frac{\Xi_{b}}{\tau_{b}^{\lambda+2}} \sim \tau_{b}^{3 / 2}+\frac{\Xi_{b}}{\tau_{b}^{\lambda}}+\ldots
\end{aligned}
$$


which shows that any loop correction to the Kähler potential of the form $\Xi / \tau_{b}^{\lambda}$ leads to a subleading contribution to $V_{\mathrm{np} 1}$ in the large volume expansion. As usual, the ellipsis stands for terms that are even more subleading in the $\tau_{b}$ expansion.

To understand the $\mathcal{E}_{s}^{K}$ correction to $G^{\bar{s} s}$ we need to consider

$$
K=-2 \ln (\mathcal{V})+\tilde{\delta} K(T, \bar{T}) \equiv-2 \ln (\mathcal{V})+\frac{\Xi_{b} g\left(T_{s}, \bar{T}_{s}\right)}{\left(T_{b}+\bar{T}_{b}\right)^{\lambda}}
$$

for some function $g\left(T_{s}, \bar{T}_{s}\right)$ of the small Kähler modulus and we assume $\lambda \geq 3 / 2$ in the following. Then, again very schematically, the scaling behavior is given by ${ }^{3}$

$$
\begin{aligned}
G^{\bar{s} s} & \simeq G_{0}^{\bar{s} s}-G_{0}^{\bar{s} i} \tilde{\delta} K_{i \bar{\jmath}} G_{0}^{\bar{\jmath} s}+\ldots \\
& \sim \tau_{b}^{3 / 2}+\Xi_{b}\left(\tau_{b}, \tau_{b}^{3 / 2}\right)\left(\begin{array}{cc}
\tau_{b}^{-\lambda-2} & \tau_{b}^{-\lambda-1} \\
\tau_{b}^{-\lambda-1} & \tau_{b}^{-\lambda}
\end{array}\right)\left(\begin{array}{c}
\tau_{b} \\
\tau_{b}^{3 / 2}
\end{array}\right)+\ldots \\
& \sim \tau_{b}^{3 / 2}+\tau_{b}^{-\lambda}+\tau_{b}^{-\lambda+3 / 2}+\tau_{b}^{-\lambda+3}+\ldots
\end{aligned}
$$

One sees that $\lambda=3 / 2$ indeed contributes at the same order as $G_{0}^{\bar{s} s}$. This is confirmed by the dependence of $G^{\bar{s} s}$ in (D.10) on $\mathcal{E}_{s}^{K}$ through $\Delta$, cf. (6.29).

We now consider $V_{\text {np2 }}$. This is proportional to $G^{\bar{\jmath} s} K_{\bar{\jmath}}$. Again we start by considering a correction to the Kähler potential whose only dependence on the Kähler moduli is via $\tau_{b}$, as in (D.39). Schematically, we find

$$
\begin{aligned}
G^{\bar{\jmath} s} K_{\bar{\jmath}} & \simeq\left(G_{0}^{\bar{\jmath} s}-G_{0}^{\bar{\jmath} b} \delta K_{b \bar{b}} G_{0}^{\bar{b} s}\right)\left[K_{\bar{\jmath}}^{(0)}+\delta K_{\bar{\jmath}}\right]+\ldots \\
& \sim \tau_{s}+\frac{G_{0}^{\bar{s} b} \Xi_{b}}{\tau_{b}^{\lambda+1}}+\ldots \sim \tau_{b}^{0}+\frac{\Xi_{b}}{\tau_{b}^{\lambda}}+\ldots
\end{aligned}
$$

This result is confirmed by the absence of $\mathcal{E}_{b}^{K}$ in the leading term of $V_{\text {np2 }}$. A calculation very similar to the one in (D.45) shows, however, that $V_{\mathrm{np} 2}$ is modified by a correction to the Kähler potential of the form (D.44) for $\lambda=3 / 2$. It is straightforward to generalize this analysis to a more general form of the "Swiss cheese" volume, with more than one small Kähler modulus.

\section{D.4 KK spectrum with fluxes}

In this section we would like to develop some intuition on how the analysis of sections 6.3 .3 and 6.4 might change in the presence of fluxes. We will restrict the discussion to one possible effect of the fluxes, namely their influence on the KK spectrum. It is not

\footnotetext{
${ }^{3}$ For $\lambda=3 / 2$ we can still use the expansion of the inverse metric (D.41), because the correction term would also be further suppressed e.g. in the dilaton.
} 
known explicitly how closed string fluxes, which are present in LVS, would change the mass spectrum. We will consider a toy example, using an analogy to the correction arising from world volume fluxes (cf. [181]), in order to get a feeling for what kind of effects one might expect. In particular, for the purposes of this appendix we assume a modified KK mass spectrum of the form

$$
m_{\mathrm{KK}}^{2} \sim \frac{1}{t_{\mathrm{str}}\left(1+\frac{F^{2}}{t_{\mathrm{str}}^{2}}\right)}=\frac{\sqrt{S_{1}}}{t\left(1+\frac{F^{2} S_{1}}{t^{2}}\right)}
$$

where $F$ represents any of the fluxes that may be present, and in the second equality the factors of $S_{1}$ appeared when expressing the 2-cycle volumes in Einstein frame as compared to the string frame $\left(t \sim e^{-\Phi / 2} t_{\text {str }}\right)$. Note that expanding (D.47) for large values of $t$ would lead to a correction $\Delta m^{2} \sim F^{2} / t^{3}$, whose scaling with the flux and with $t$ is reminiscent of the moduli masses induced by closed string 3-form flux [202, 203]. In that case, the suppression would be by the overall volume (which would lead to only mild effects in LVS), but in (D.47) we allow for a suppression by single 2-cycle volumes (which might be the small 2-cycle in the $\mathbb{P}_{[1,1,1,6,9]}^{4}$ model).

Substituting (D.47) in 6.23), we now consider the scalar potential resulting from

$$
\begin{aligned}
K & =-\ln \left(2 S_{1}\right)-2 \ln (\mathcal{V})+K_{\mathrm{cs}}(u, \bar{u})-\frac{\tilde{\xi} S_{1}^{3 / 2}}{\mathcal{V}}+\frac{\sqrt{\tau_{b}} \mathcal{E}_{b}^{K}}{S_{1} \mathcal{V}}+\frac{\sqrt{\tau_{s}} \mathcal{E}_{s}^{K}}{S_{1} \mathcal{V}}\left(1+\frac{F^{2} S_{1}}{\tau_{s}}\right) \\
W & =W_{0}+A e^{-a T_{s}}
\end{aligned}
$$

We have not included any flux correction to the term proportional to $\mathcal{E}_{b}^{K}$ because we expect such corrections to be subleading in a large volume expansion.4 Note that the $F$-dependent correction term we did include is of the same form as the winding string correction $\sim \mathcal{E}_{s}^{W}$, when one neglects any potential flux corrections to the winding string spectrum, cf. 6.24) (remember that $g_{\mathrm{W}}^{s}$ would just be proportional to $1 / \sqrt{\tau_{s}}$ without fluxes). Thus, by considering (D.48) we implicitly also analyze in the following the effect of corrections from winding strings (recall from section 6.3.3 that this correction is not present in the $\mathbb{P}_{[1,1,1,6,9]}^{4}$ model, but may be present in general).

We now give the generalization of (6.26)- 6.28$)$ when using the modified Kähler potential (D.48). The three contributions at leading order $\left(\mathcal{O}\left(\mathcal{V}^{-3}\right)\right)$ in the large volume expansion

\footnotetext{
${ }^{4}$ Even though we think it is unlikely, we can not exclude that the correction to KK masses that scale like $t_{b}^{-1}$ without fluxes is only suppressed by $F^{2} / \tau_{s}$ instead of $F^{2} / \tau_{b}$. In that case, one would have to redo the analysis of appendix D.3.5, using (D.44) with $\lambda=1$. This would prohibit the use of the expansion (D.41), because in the large volume limit the leading contribution to $G_{s \bar{s}}$ would arise from the loop correction (it would scale as $\tau_{b}^{-1}$ as opposed to the scaling of the tree level contribution $\sim \tau_{b}^{-3 / 2}$ ). In that case the leading terms in $V_{\mathrm{np} 1}$ and $V_{\mathrm{np} 2}$ would be suppressed compared to $V_{3}$ and only arise at order $\mathcal{V}^{-10 / 3}$, thus invalidating the volume expansion of LVS.
} 
are

$$
\begin{aligned}
V_{\mathrm{np} 1} & =e^{K_{\mathrm{cs}}} \frac{24 a^{2}|A|^{2} \tau_{s}^{3 / 2} e^{-2 a \tau_{s}}}{\mathcal{V} \Delta} \\
V_{\mathrm{np} 2} & =-e^{K_{\mathrm{cs}}} \frac{2 a\left|A W_{0}\right| \tau_{s} e^{-a \tau_{s}}}{S_{1} \mathcal{V}^{2}}\left[1+\frac{6 \mathcal{E}_{s}^{K}}{\Delta}\left(1-2 \frac{F^{2} S_{1}}{\tau_{s}}\right)\right] \\
V_{3} & =\frac{3 e^{K_{\mathrm{cs}}}\left|W_{0}\right|^{2}}{8 \mathcal{V}^{3}}\left[S_{1}^{1 / 2} \tilde{\xi}+\right. \\
& \left.+\frac{\sqrt{\tau_{s}}\left(4\left(\mathcal{E}_{s}^{K}\right)^{2}-8\left(\mathcal{E}_{s}^{K}\right)^{2} F^{2} S_{1} \tau_{s}^{-1}\left(1+F^{2} S_{1} \tau_{s}^{-1}\right)-\frac{8 \sqrt{2}}{3} F^{2} S_{1}^{2} \mathcal{E}_{s}^{K}\right)}{S_{1}^{2} \Delta}\right]
\end{aligned}
$$

where the axion has already been minimized for, as discussed in section D.1.2, and now $\Delta$ is generalized to

$$
\Delta \equiv \sqrt{2} S_{1} \tau_{s}-3 \mathcal{E}_{s}^{K}\left(1-3 \frac{F^{2} S_{1}}{\tau_{s}}\right)
$$

Plots for $F=1$ and $F=3$ are given in fig. D.1, and they look quite similar to the plot without flux, fig. 6.4. Qualitatively, the conclusion is the same; only for nongeneric values of the $g_{\mathrm{s}}$ corrections do they compete with the $\alpha^{\prime}$ correction. Note, however, that the amount of fine-tuning seems to depend on the value of the flux, cf. fig. D.1. The same is true for the dependence of the values of $\mathcal{V}$ and $\tau_{s}$ at the minimum on $S_{1}$ and $\mathcal{E}_{s}^{K}$. For $F=3$, for instance, this dependence becomes more complicated than what we found in (6.31). For the parameter range shown in fig. D.1, the values of $\tau_{s}$ and $\mathcal{V}$ in the minimum vary in the ranges $\tau_{s} \in[14.6,46.3]$ and $\log _{10} \mathcal{V} \in[3.7,15.5]$, where the smallest value for both of them is reached in the corner where the two corrections become comparable.

Also the cancellation that we found for the gaugino masses survives the inclusion of the flux factor in (D.48). The correction still only appears at sub-sub-leading order in an expansion in $\ln \left(1 / m_{3 / 2}\right)$ and we find (again using the dilute flux approximation for the prefactor $\left.(\operatorname{Re} f)^{-1}\right)$ :

$$
\begin{aligned}
\left|M_{s}\right| & =\left|\frac{F^{s}}{2 \tau_{s}}\right|=3 e^{K / 2}\left|W_{0}\right| \frac{1}{4 a \tau_{s}}+\frac{1}{16 a^{2} \tau_{s}^{2}}+\frac{S_{1}-12 \sqrt{2} a\left(1-2 F^{2} S_{1} \tau_{s}^{-1}\right) \mathcal{E}_{s}^{K}}{64 S_{1} a^{3} \tau_{s}^{3}}+\ldots \mid \\
& \sim \frac{m_{3 / 2}}{\ln \left(1 / m_{3 / 2}\right)}\left[1+\mathcal{O}\left(\frac{1}{\ln \left(1 / m_{3 / 2}\right)}\right)\right]
\end{aligned}
$$

This concludes our brief study of the direct effects of fluxes on the loop corrections. 

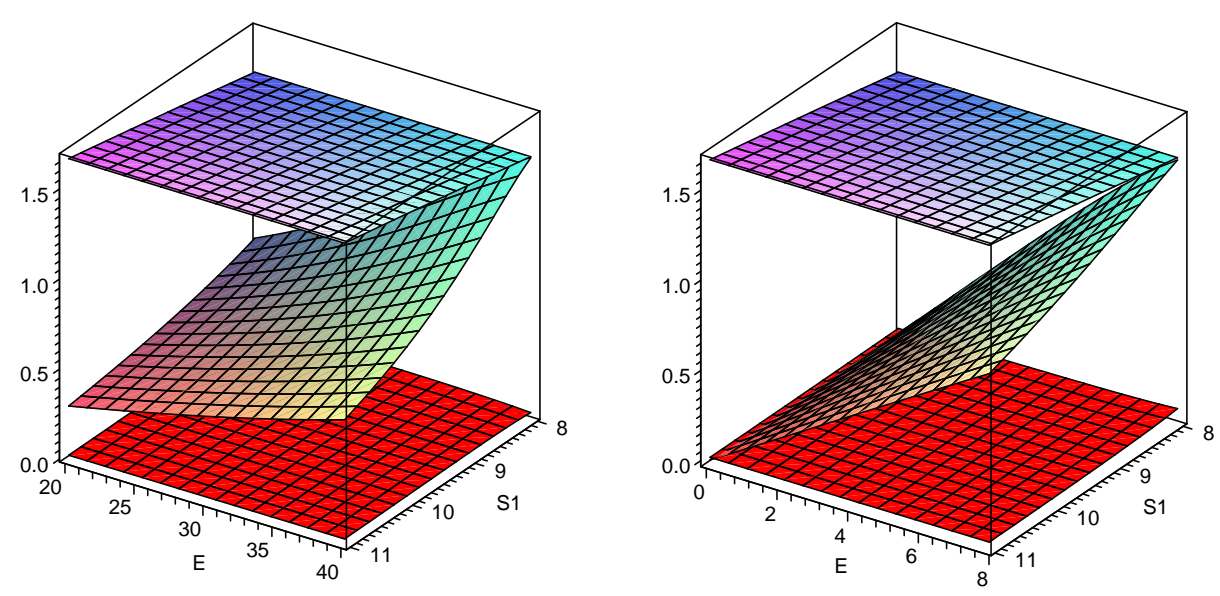

Figure D.1: Similarly to figure 6.4, the top surface is the $\alpha^{\prime}$ correction, the second is the $g_{\mathrm{s}}$ correction (with $F=1$ in the left graph and $F=3$ in the right), and the "red carpet" is $10 / \Delta$, with $\Delta$ from (D.52), using the same values as in fig. 6.4. The result is qualitatively the same as before. Note, however, that the range for $\mathcal{E}_{s}^{K}$ differs. For larger values of $F$ one does not need to fine-tune $\mathcal{E}_{s}^{K}$ as much in order for the two corrections to become of similar order.

\section{D.5 The orientifold calculation}

In the main text, we are interested in how $\Delta K_{g_{\mathrm{s}}}$, the one-loop correction to the Kähler potential, scales with the Kähler moduli $T_{i}$. Our argument in section 6.3.1 is based on the known result for $\Delta K_{g_{\mathrm{s}}}$ in the case of $\mathcal{N}=2$ supersymmetric $K 3 \times T^{2}$ orientifolds and $\mathcal{N}=1$ supersymmetric $T^{6} / \mathbb{Z}_{N}$ (or $T^{6} /\left(\mathbb{Z}_{2} \times \mathbb{Z}_{2}\right)$ ) orientifolds, from [171] (see also [70]). Here we review this computation for the case of $K 3 \times T^{2}$, and take this opportunity to adapt it to our case of D3-branes and D7-branes from the beginning. (One can also obtain them by T-duality on the final D9/D5 results of [171], e.g. as in the appendix of [204], but as we shall see, the direct computation is enlightening in its own right.) We will leave out details that are essentially identical to [171, and only emphasize the differences.

As shown in 171 using "Kähler adapted" vertex operators, the easiest way to compute $\Delta K_{g_{\mathrm{s}}}$ is by considering the 2-point function of the complex structure modulus $u$ of $T^{2}$, with vanishing Wilson line moduli, i.e.

$$
\left\langle V_{u} V_{\bar{u}}\right\rangle=-\sum_{\sigma} \frac{4 g_{c}^{2} \alpha^{\prime-4}}{(u-\bar{u})^{2}}\left\langle V_{Z Z}^{(0,0)} V_{\overline{Z Z}}^{(0,0)}\right\rangle_{\sigma} .
$$

Here, we use the notation of [171],

$$
\begin{aligned}
V_{u}^{(0,0)} & =-g_{c} \alpha^{\prime-2} \frac{2}{u-\bar{u}} V_{Z Z}^{(0,0)}, \\
V_{\bar{u}}^{(0,0)} & =g_{c} \alpha^{\prime-2} \frac{2}{u-\bar{u}} V_{\overline{Z Z}}^{(0,0)}
\end{aligned}
$$


and

$$
\begin{aligned}
V_{Z Z}^{(0,0)} & =-\frac{2}{\alpha^{\prime}} \int_{\Sigma} d^{2} z\left[i \partial Z+\frac{1}{2} \alpha^{\prime}(p \cdot \psi) \Psi\right]\left[i \bar{\partial} Z+\frac{1}{2} \alpha^{\prime}(p \cdot \tilde{\psi}) \tilde{\Psi}\right] e^{i p X} \\
V_{\overline{Z Z}}^{(0,0)} & =-\frac{2}{\alpha^{\prime}} \int_{\Sigma} d^{2} z\left[i \partial \bar{Z}+\frac{1}{2} \alpha^{\prime}(p \cdot \psi) \bar{\Psi}\right]\left[i \overline{\partial \bar{Z}}+\frac{1}{2} \alpha^{\prime}(p \cdot \tilde{\psi}) \overline{\tilde{\Psi}}\right] e^{i p X}
\end{aligned}
$$

As in [171], and [205] before that, we find these complex worldsheet variables particularly convenient:

$$
\begin{gathered}
Z=\sqrt{\frac{\sqrt{G_{\mathrm{str}}}}{2 u_{2}}}\left(X^{4}+\bar{u} X^{5}\right), \quad \bar{Z}=\sqrt{\frac{\sqrt{G_{\mathrm{str}}}}{2 u_{2}}}\left(X^{4}+u X^{5}\right), \\
\Psi=\sqrt{\frac{\sqrt{G_{\mathrm{str}}}}{2 u_{2}}}\left(\psi^{4}+\bar{u} \psi^{5}\right), \quad \bar{\Psi}=\sqrt{\frac{\sqrt{G_{\mathrm{str}}}}{2 u_{2}}}\left(\psi^{4}+u \psi^{5}\right),
\end{gathered}
$$

where $\sqrt{G_{\text {str }}}$ is the volume of $T^{2}$ measured in string frame. The 2-point function (D.54) can be expanded for small momenta, $p_{1} \cdot p_{2} \ll 1$, and we obtain

$$
\begin{aligned}
& \left\langle V_{Z Z}^{(0,0)} V_{\overline{Z Z}}^{(0,0)}\right\rangle_{\sigma}=-V_{4} \frac{\left(p_{1} \cdot p_{2}\right){\sqrt{G_{\mathrm{str}}}}^{-1}}{16\left(4 \pi^{2} \alpha^{\prime}\right)^{2}} \int_{0}^{\infty} \frac{d t}{t^{4}} \int_{\mathcal{F}_{\sigma}} d^{2} \nu_{1} d^{2} \nu_{2} \\
& \times \sum_{k=0,1} \sum_{\mathbf{n}=(n, m)^{T}} \operatorname{tr}\left[e^{-\pi \mathbf{n}^{T} G_{\mathrm{str}}^{-1} \mathbf{n} t^{-1}} \sum_{\substack{\alpha \beta \\
\text { even }}} \frac{\vartheta\left[\begin{array}{l}
\alpha \\
\beta
\end{array}\right](0, \tau)}{\eta^{3}(\tau)} \gamma_{\sigma, k} \mathcal{Z}_{\sigma, k}^{\mathrm{int}}\left[\begin{array}{l}
\alpha \\
\beta
\end{array}\right]\right. \\
& \times\left[\left\langle\bar{\partial} Z\left(\bar{\nu}_{1}\right) \overline{\partial \bar{Z}}\left(\bar{\nu}_{2}\right)\right\rangle_{\sigma}\left\langle\Psi\left(\nu_{1}\right) \bar{\Psi}\left(\nu_{2}\right)\right\rangle_{\sigma}^{\alpha, \beta}\left\langle\psi\left(\nu_{1}\right) \psi\left(\nu_{2}\right)\right\rangle_{\sigma}^{\alpha, \beta}\right. \\
& \left.\left.+\left\langle\bar{\partial} Z\left(\bar{\nu}_{1}\right) \partial \bar{Z}\left(\nu_{2}\right)\right\rangle_{\sigma}\left\langle\Psi\left(\nu_{1}\right) \bar{\Psi}\left(\bar{\nu}_{2}\right)\right\rangle_{\sigma}^{\alpha, \beta}\left\langle\psi\left(\nu_{1}\right) \tilde{\psi}\left(\bar{\nu}_{2}\right)\right\rangle_{\sigma}^{\alpha, \beta}+\text { c.c. }\right]\right]+\mathcal{O}\left(\left(p_{1} \cdot p_{2}\right)^{2}\right) \text {. }
\end{aligned}
$$

For the details we refer to [171]. The main difference to the corresponding formula (C.3) in [171] is the appearance of the inverse metric $G_{\text {str }}^{-1}$ in the exponent arising from the zero mode sum, and in the prefactor. This is due to the fact that the D3 and D7 branes are localized along the $T^{2}$, and so the closed string channel involves a Kaluza-Klein momentum sum instead of a winding sum. The sum over bosonic zero modes has been made explicit, since there is also an implicit dependence on $m, n$ in the bosonic correlators: this arises from the classical piece in the split into zero modes and fluctuations. That is, $Z(\nu)=$ $Z_{\text {class }}(\nu)+Z_{\mathrm{qu}}(\nu)$, where the classical part is given by

$$
Z_{\text {class }}=\sqrt{\alpha^{\prime}} \sqrt{\frac{\sqrt{G_{\text {str }}}}{2 u_{2}}}(n+m \bar{u}) \operatorname{Re}(\nu) \tilde{c}_{\sigma}, \quad \tilde{c}_{\sigma}=\left\{\begin{array}{ll}
1 & \text { for } \mathcal{K} \\
2 & \text { for } \mathcal{A}, \mathcal{M}
\end{array} .\right.
$$

These zero modes have the right periodicity under $\operatorname{Re}(\nu) \rightarrow \operatorname{Re}(\nu)+\pi($ for $\mathcal{A}, \mathcal{M})$ or $\operatorname{Re}(\nu) \rightarrow \operatorname{Re}(\nu)+2 \pi($ for $\mathcal{K})$, i.e. $X^{4} \rightarrow X^{4}+2 \pi n \sqrt{\alpha^{\prime}}$ and $X^{5} \rightarrow X^{5}+2 \pi m \sqrt{\alpha^{\prime}}$. In contrast to [171] they involve the real part of $\nu$. The reason is again that in the D3/D7 
case the branes are localized along $T^{2}$ and thus the winding appears in the open string channel as opposed to the closed string channel (as was the case for D9/D5 branes).

The sum over spin structures is performed using Riemann identities. This leaves the correlators of the bosonic fields as the only piece that depends on the positions $\nu_{i}$ of the vertex operators. The $\nu_{i}$ integral can then be evaluated. As the zero modes (D.59) involve the real part of $\nu$ in the case of D3/D7-branes, in contrast to the D9/D5-case studied in [171, the zero mode contribution in the $Z$-correlators drops out. The quantum part is evaluated using the method of images on the worldsheet [206, 207, 172. To evaluate the KK sum in (D.58), it is useful to regularize the integral over $t$ by a UV cutoff $\Lambda$. With this we obtain

$$
\begin{aligned}
& \int_{1 /\left(e_{\sigma} \Lambda^{2}\right)}^{\infty} \frac{d t}{t^{4}} \sum_{\mathbf{n}=(n, m)^{T}}^{\prime}\left[\pi^{3} c_{\sigma}^{2} t \alpha^{\prime} e^{-\pi \mathbf{n}^{T} G_{\mathrm{str}}^{-1} \mathbf{n} t^{-1}}\right] \\
& =\frac{1}{2} \pi^{3} \alpha^{\prime} c_{\sigma}^{2} e_{\sigma}^{2} \Lambda^{4}+\pi \alpha^{\prime} c_{\sigma}^{2}{\sqrt{G_{\mathrm{str}}}}^{2} E_{2}(0, u)+\ldots
\end{aligned}
$$

where the prime at the sum indicates that the $(n, m)=(0,0)$ term is left out, and $c_{\sigma}$, $e_{\sigma}$ are constants whose precise values will not be important in the following (but can be found in [171]). Terms that go to zero in the limit $\Lambda \rightarrow \infty$ have been dropped, as indicated by the ellipsis. The nonholomorphic Eisenstein series $E_{2}(0, u)$ is the $s=2$ special case of

$$
E_{s}(0, u)=\sum_{\mathbf{n}=(n, m)^{T}}^{\prime} \frac{u_{2}^{s}}{|n+m u|^{2 s}} .
$$

The terms involving the UV cutoff $\Lambda$ drop out after summing over all diagrams, due to tadpole cancellation. We have then reduced (D.58) to

$$
\begin{aligned}
&\left\langle V_{Z Z}^{(0,0)} V_{\overline{Z Z}}^{(0,0)}\right\rangle_{\sigma}=-\left(p_{1} \cdot p_{2}\right) \alpha^{\prime} \frac{V_{4}}{\left(4 \pi^{2} \alpha^{\prime}\right)^{2}} \frac{c_{\sigma}^{2} \pi \sqrt{G_{\text {str }}}}{8} \sum_{k} \operatorname{tr}\left[E_{2}(0, u) \gamma_{\sigma, k} \mathcal{Q}_{\sigma, k}\right] \\
&+\mathcal{O}\left(\left(p_{1} \cdot p_{2}\right)^{2}\right) .
\end{aligned}
$$

The quantities $\mathcal{Q}_{\sigma, k}$ come from the sum over spin structures and are defined in [171. Introducing the notation

$$
\mathcal{E}_{2}(0, u)=\sum_{\sigma} c_{\sigma}^{2} \sum_{k=0,1} \operatorname{tr}\left[E_{2}(0, u) \gamma_{\sigma, k} \mathcal{Q}_{\sigma, k}\right],
$$

we end up with (neglecting some irrelevant factors of $g_{c}, \alpha^{\prime}$, terms subleading in the lowenergy expansion, and constants of order 1)

$$
\left\langle V_{u} V_{\bar{u}}\right\rangle \sim-i\left(p_{1} \cdot p_{2}\right) \frac{V_{4}}{\left(4 \pi^{2} \alpha^{\prime}\right)^{2}} \frac{\sqrt{G_{\text {str }}}}{(u-\bar{u})^{2}} \mathcal{E}_{2}(0, u) .
$$


To read off the one-loop correction to the kinetic term of $u$ we need to perform a Weyl rescaling to the Einstein frame. In the one-loop term (D.64) this just leads to

$$
\text { Weyl rescaling: } \quad \times \frac{e^{2 \Phi}}{\mathcal{V}^{\text {str }}},
$$

where

$$
\mathcal{V}^{\text {str }}=\mathcal{V}_{\mathrm{K} 3}^{\mathrm{str}} \sqrt{G_{\mathrm{str}}}
$$

is the overall volume in string frame. The Kähler potential can then be read off from the kinetic term by use of the identity

$$
\partial_{u} \partial_{\bar{u}} E_{2}(0, u)=-\frac{2}{(u-\bar{u})^{2}} E_{2}(0, u),
$$

producing the final result

$$
\Delta K_{g_{\mathrm{s}}} \sim \frac{\sqrt{G_{\mathrm{str}}} e^{\Phi}}{\mathcal{V}^{\operatorname{str}}(S+\bar{S})} \mathcal{E}_{2}(0, u)
$$

where $\sqrt{G_{\text {str }}} e^{\Phi} / \mathcal{V}^{\text {str }}$ is to be interpreted as a function of the Kähler variables. In the $K 3 \times T^{2}$ orientifold case, using (D.66), this is just proportional to $e^{\Phi} / \mathcal{V}_{\mathrm{K} 3}^{\mathrm{str}} \sim(T+\bar{T})^{-1}$ (with Re $T$ the volume of $K 3$ measured in Einstein frame), giving a result T-dual to [171] (note that we switched the real and imaginary parts in the definition of $T$ and $S$ as compared to [171], to conform with the rest of this work). As we argue in section 6.3.1, in general the dependence on the Kähler moduli will be more complicated than this, because there is no analog to the relation (D.66). It is still clear that the inverse suppression in the overall volume will appear as in (D.68), given that it is a direct consequence of the Weyl rescaling.

\section{D.6 Factorized approximation}

As mentioned in section 6.4.2, it is an important issue to what extent the moduli spaces of Kähler and complex structure moduli factorize. In this appendix, we give further details on the factorized approximation.

A common starting point in the analysis of the potential arising in type IIB theory with 3 -form fluxes is to assume that all complex structure moduli $u^{\alpha}$ and the dilaton $S$ are stabilized by demanding

$$
D_{u^{\alpha}} W=0=D_{S} W .
$$

In this case the F-term potential for the moduli (6.7) reduces to

$$
V=e^{K}\left(G^{\bar{\jmath} i} D_{\bar{\jmath}} \bar{W} D_{i} W-3|W|^{2}\right),
$$


where as in the main text, the indices $i$ and $j$ refer only to the Kähler moduli and thus run from 1 to $h^{1,1}$. Note that even though the complex structure moduli and the dilaton are assumed to be stabilized by (D.69), the inverse metric $G^{\bar{j} i}$ is part of the inverse of the whole moduli space metric. More precisely, if we denote the Kähler moduli by $T_{i}$, as before, and all other moduli (i.e. the complex structure moduli and the dilaton) collectively as $Z^{a}$, the moduli space metric is given by

$$
G_{I \bar{J}} \sim\left(\begin{array}{ll}
K_{i \bar{\jmath}} & K_{i \bar{b}} \\
K_{a \bar{\jmath}} & K_{a \bar{b}}
\end{array}\right)
$$

We denote the inverse of this (whole) metric by $G^{\bar{J} I}$. In general

$$
G^{\bar{\jmath} i} \neq\left(K_{i \bar{\jmath}}\right)^{-1}
$$

Equality only holds if $G_{i \bar{b}}=0$, i.e. if the moduli space of the Kähler moduli is factorized from the rest, as it is the case without loop and $\alpha^{\prime}$ corrections.

In this appendix, we would like to investigate at which order in a large volume expansion the two matrices in (D.72) start to deviate from each other. For this analysis we assume a volume of the "Swiss cheese" form as in (6.48) and a Kähler potential of the form (6.24) (without taking possible effects of fluxes on the KK and winding mode spectra into account as was done in appendix D.4 thus, $g_{\mathrm{K}}^{\mathfrak{a}} \sim t_{\mathfrak{a}}$ and $g_{\mathrm{K}}^{\mathfrak{q}} \sim t_{\mathfrak{q}}^{-1}$ for some 2-cycle volumes). To avoid cumbersome notation we will indicate all the small moduli collectively as $\tau_{s}$. We then use the formula

$$
\left(\begin{array}{cc}
A & B \\
C & D
\end{array}\right)^{-1}=\left(\begin{array}{cc}
A^{-1}\left(1+B P^{-1} C A^{-1}\right) & -A^{-1} B P^{-1} \\
-P^{-1} C A^{-1} & P^{-1}
\end{array}\right)
$$

where $P$ is the Schur complement of $A$, defined as

$$
P=D-C A^{-1} B
$$

In our case $P$ is the Schur complement of $K_{i \bar{\jmath}}$. From 6.24 we read off that

$$
G_{I \bar{J}} \sim\left(\begin{array}{cccc}
\tau_{b}^{-2} & \tau_{b}^{\beta} & \tau_{b}^{-2} & \tau_{b}^{-2} \\
\tau_{b}^{\beta} & \tau_{b}^{-3 / 2} & \tau_{b}^{-3 / 2} & \tau_{b}^{-3 / 2} \\
\tau_{b}^{-2} & \tau_{b}^{-3 / 2} & \tau_{b}^{0} & \tau_{b}^{-1} \\
\tau_{b}^{-2} & \tau_{b}^{-3 / 2} & \tau_{b}^{-1} & \tau_{b}^{0}
\end{array}\right)
$$

where we only indicate the $\tau_{b}$ dependence and the indices run over $I, J=\left\{T_{b}, T_{s}, u, S\right\}$. Here $\beta=-2$ for those $\tau_{i}$ with a nonvanishing $a_{i}$ in (6.48) (so $\beta$ has an implicit index $i$ ), otherwise $\beta=-5 / 2$ (which is in particular the value in the $\mathbb{P}_{[1,1,1,6,9]}$ case). We decompose 
$G_{I \bar{J}}$ as in D.73

$$
\begin{array}{rlrl}
A & \sim\left(\begin{array}{cc}
\tau_{b}^{-2} & \tau_{b}^{\beta} \\
\tau_{b}^{\beta} & \tau_{b}^{-3 / 2}
\end{array}\right), & & A^{-1} \sim\left(\begin{array}{cc}
\tau_{b}^{2} & \tau_{b}^{7 / 2+\beta} \\
\tau_{b}^{7 / 2+\beta} & \tau_{b}^{3 / 2}
\end{array}\right), \\
B=C^{T} \sim\left(\begin{array}{cc}
\tau_{b}^{-2} & \tau_{b}^{-2} \\
\tau_{b}^{-3 / 2} & \tau_{b}^{-3 / 2}
\end{array}\right), & D \sim\left(\begin{array}{cc}
\tau_{b}^{0} & \tau_{b}^{-1} \\
\tau_{b}^{-1} & \tau_{b}^{0}
\end{array}\right), \\
& P \sim\left(\begin{array}{cc}
\tau_{b}^{0} & \tau_{b}^{-1} \\
\tau_{b}^{-1} & \tau_{b}^{0}
\end{array}\right), & & P^{-1} \sim\left(\begin{array}{cc}
\tau_{b}^{0} & \tau_{b}^{-1} \\
\tau_{b}^{-1} & \tau_{b}^{0}
\end{array}\right) .
\end{array}
$$

Using (D.73) one easily obtains the scaling of the inverse:

$$
G^{\bar{J} I} \sim\left(\begin{array}{cccc}
\tau_{b}^{2} & \tau_{b}^{7 / 2+\beta} & \tau_{b}^{0} & \tau_{b}^{0} \\
\tau_{b}^{7 / 2+\beta} & \tau_{b}^{3 / 2} & \tau_{b}^{0} & \tau_{b}^{0} \\
\tau_{b}^{0} & \tau_{b}^{0} & \tau_{b}^{0} & \tau_{b}^{-1} \\
\tau_{b}^{0} & \tau_{b}^{0} & \tau_{b}^{-1} & \tau_{b}^{0}
\end{array}\right)
$$

Now, from (D.73), $G^{\bar{\jmath} i}$ receives two contributions. The first is $K^{\bar{j} i}$, that would be the only term in the case of a factorized metric; the second is $K_{h \bar{\jmath}}^{-1} K_{h \bar{b}} P_{a \bar{b}}^{-1} K_{a \bar{l}} K_{i \bar{l}}^{-1}$, that breaks factorization. Let us compare their $\tau_{b}$ scaling:

$$
\begin{aligned}
G^{\bar{\jmath} i} & =A^{-1}+A^{-1} B P^{-1} C A^{-1} \\
& \sim\left(\begin{array}{cc}
\tau_{b}^{2} & \tau_{b}^{7 / 2+\beta} \\
\tau_{b}^{7 / 2+\beta} & \tau_{b}^{3 / 2}
\end{array}\right)+\left(\begin{array}{cc}
\tau_{b}^{0} & \tau_{b}^{0} \\
\tau_{b}^{0} & \tau_{b}^{0}
\end{array}\right) .
\end{aligned}
$$

Thus the corrections coming from non-vanishing off-diagonal metric elements in (D.71) set in with a suppression by $\tau_{b}^{-2}, \tau_{b}^{-7 / 2-\beta}$ and $\tau_{b}^{-3 / 2}$ in $G^{\bar{b} b}, G^{\bar{b} s}$ and $G^{\bar{s} s}$, respectively. In the explicit example based on $\mathbb{P}_{[1,1,1,6,9]}, \beta=-5 / 2$, and we checked this result by comparing to the subleading terms in (D.10).

\section{D.6.1 Factorized approximation of the scalar potential}

What we are really interested in is not the (inverse) metric itself, but the scalar potential, to which we now turn. For the nonperturbative terms $V_{\mathrm{np} 1}$ and $V_{\mathrm{np} 2}$, the suppression of the off-diagonal terms in (D.78) is inherited by the scalar potential, as they are proportional to $G^{\bar{s} s} \bar{W}_{, \bar{s}} W_{, s}$ and $G^{\bar{\jmath} s} \bar{K}_{\bar{\jmath}}$, respectively. For $V_{3}$ things are not as simple, due to the no-scale structure at leading order. Let us neglect for a moment all the quantum corrections, then the no-scale structure implies

$$
\begin{aligned}
{\left[G^{\overline{\overline{ } j}} K_{\bar{\imath}} K_{j}-3\right]_{\mathrm{no}-\mathrm{scale}} } & \sim\left(\tau_{b}^{-1}, \tau_{b}^{\beta+1}\right)\left(\begin{array}{cc}
\tau_{b}^{2} & \tau_{b}^{7 / 2+\beta} \\
\tau_{b}^{7 / 2+\beta} & \tau_{b}^{3 / 2}
\end{array}\right)\left(\begin{array}{c}
\tau_{b}^{-1} \\
\tau_{b}^{\beta+1}
\end{array}\right)-3 \\
& \sim \tau_{b}^{0}+\tau_{b}^{2 \beta+7 / 2}=0 .
\end{aligned}
$$


The two terms have to vanish independently. Now let us add corrections that break no-scale structure. Because of the cancellation described in appendix D.3.3, the leading contribution can be seen to come at order $\tau_{b}^{-3 / 2}$ (from the $\alpha^{\prime}, \mathcal{E}_{s}^{K}$ and $\mathcal{E}_{s}^{W}$ corrections). On the other hand, the off-diagonal terms appear at order

$$
\begin{aligned}
{\left[G^{\bar{i} j} K_{\bar{\imath}} K_{j}\right]_{\text {off-diagonal }} } & \sim\left(\tau_{b}^{-1}, \tau_{b}^{\beta+1}\right)\left(\begin{array}{cc}
\tau_{b}^{0} & \tau_{b}^{0} \\
\tau_{b}^{0} & \tau_{b}^{0}
\end{array}\right)\left(\begin{array}{c}
\tau_{b}^{-1} \\
\tau_{b}^{\beta+1}
\end{array}\right) \\
& \sim \tau_{b}^{-2}+\tau_{b}^{\beta}+\tau_{b}^{2 \beta+2} \\
& \sim \tau_{b}^{-2}+\ldots
\end{aligned}
$$

for both $\beta=-2$ and $\beta=-5 / 2$. Therefore, the off-diagonal terms of the moduli space metric appear in the scalar potential with a suppression of at least $\tau_{b}^{-1 / 2}$ (as is confirmed by the explicit example of section 6.3.3, cf. formulas (6.26)-(6.30)). The suppression can be even stronger if some corrections are absent and the leading term in (D.80) vanishes.

To summarize: if one is only interested in the leading term of the scalar potential in the large volume (i.e. large $\tau_{b}$ ) expansion, then one can use the factorized approximation, i.e.

$$
G^{\bar{\jmath} i}=K^{\bar{\jmath} i}+\mathcal{O}\left(\tau_{b}^{0}\right)
$$

This provides a useful tool to simplify the calculations. 


\section{Symbols Used in the Paper}

\begin{tabular}{|c|c|c|c|}
\hline Var. & Mass dim. & Description & Definition \\
\hline$\overline{M_{P l}}$ & 1 & reduced Planck mass & $\bar{M}_{P l} \equiv\left(8 \pi G_{N}\right)^{-1 / 2}$ \\
\hline$\kappa_{4}^{2}$ & -2 & & $\kappa_{4}^{2}=M_{P l}^{-2}=8 \pi G_{N}$ \\
\hline$z_{A}$ & $-3 / 2$ & complex conifold coordinates & $\sum_{A} z_{A}^{2}=0$ \\
\hline$x_{A}$ & $-3 / 2$ & & $x_{A}=\Re z_{A}$ \\
\hline$y_{A}$ & $-3 / 2$ & & $y_{A}=\Im z_{A}$ \\
\hline$z_{23}$ & $-3 / 2$ & & $z_{23} \equiv z_{2}^{2}+z_{3}^{2}$ \\
\hline$x_{23}$ & $-3 / 2$ & & $x_{23} \equiv x_{2}^{2}+x_{3}^{2}$ \\
\hline$\varepsilon$ & $-3 / 2$ & conifold deformation parameter & $\sum_{i} z_{i}^{2}=\varepsilon$ \\
\hline$\mu$ & $-3 / 2$ & embedding parameter & $g\left(z_{1}\right)=\left(1-z_{1} / \mu\right)$ \\
\hline$r$ & -1 & radial coordinate on the conifold & $r^{3}=\sum_{i}\left|z_{i}\right|^{2}$ \\
\hline$K$ & 2 & moduli Kähler potential & $\kappa^{2} \mathcal{K}=-3 \log U$ \\
\hline$k$ & -2 & conifold Kähler potential & \\
\hline$W$ & 3 & superpotential & 4.3 \\
\hline$W_{0}$ & 3 & GVW-flux superpotential & $W_{0}=\int G \wedge \Omega$ \\
\hline$W_{\mathrm{np}}$ & 3 & non-perturbative superpotential & $W_{\mathrm{np}}=A\left(z_{i}\right) e^{-a T}$ \\
\hline$A_{0}$ & 3 & prefactor of $W_{\mathrm{np}}$ & $A_{0}=A\left(z_{i}=0\right)$ \\
\hline$V_{F}$ & 4 & F-term potential & 2.34 \\
\hline$V(\phi)$ & 4 & inflaton potential & $4.58),(5.31)$ \\
\hline$V_{\text {up }}$ & 4 & uplifting term & 5.17 \\
\hline$\beta$ & 0 & uplifting parameter & $(4.8),(5.21)$ \\
\hline$\Lambda$ & 4 & cosmological constant & 4.58 5.23 \\
\hline$B$ & 4 & term in the potential & 5.24 \\
\hline$C$ & 4 & term in the potential & 5.25 \\
\hline$U$ & 0 & argument of Kähler potential & $U=T+\bar{T}-\gamma k$ \\
\hline$\gamma$ & 2 & in sec. 5.3 and 5.4 . factor in $K$ & $\gamma \equiv \tau_{0} T_{3} /\left(3 M_{P l}^{2}\right)$ \\
\hline$\gamma$ & 0 & in sec. $\overline{5.5}$ and $\overline{5.6}$; inflaton Lorenz factor & $\gamma \equiv\left(1-f \dot{\phi}^{2}\right)^{-1 / 2}$ \\
\hline$T_{3}$ & 4 & D3-brane tension & $\left(\alpha^{\prime}\right)^{-2}(2 \pi)^{-3} g_{s}^{-1}$ \\
\hline$T$ & 0 & complex Kähler modulus & \\
\hline$\tau$ & 0 & real part of $T$ & $2 \tau=T+\bar{T}$ \\
\hline$g(z)$ & 0 & embedding equation & $A\left(z_{i}\right) \propto(g(z))^{1 / n}$ \\
\hline$\tilde{g}$ & 0 & Kuperstein embedding's function & $g(z)=\tilde{g}\left(z_{23}\right)-z_{1}$ \\
\hline$d$ & 1 & canonical normalization prefactor & $\varepsilon^{2 / 3} \sqrt{T_{3} c}$ \\
\hline$c$ & 0 & numerical factor in $k$ & $2^{1 / 6} / 3^{1 / 3} \simeq 0.77$ \\
\hline
\end{tabular}




\begin{tabular}{ccll}
\hline Var. & Mass dim. & Description & Definition \\
\hline \hline $\mathcal{V}$ & 0 & volume of the Calabi-Yau manifold & $(\overline{D .18}),(\mathrm{D} .27)$ \\
$\tau_{b}$ & 0 & big 4-cycle volume of $\mathbb{P}_{[1,1,1,6,9]}^{4}$ & $(6.14)$ \\
$\tau_{s}$ & 0 & small 4-cycle volume of $\mathbb{P}_{[1,1,1,6,9]}^{4}$ & $S \equiv e^{-\phi}+i C_{0}$ \\
$S$ & 0 & axio-dilaton & \\
$u$ & 0 & complex structure moduli & \\
$m_{3 / 2}$ & 1 & gravitino mass & \\
$M_{a}$ & 1 & gaugino mass & $6.35)$ \\
$f_{a}$ & 0 & gauge kinetic function & $(6.36)$ \\
$n$ & 0 & number of D7-branes & \\
$N_{D 3}$ & 0 & number of D3-branes at the tip & \\
$N_{e}$ & 0 & number of e-foldings & $(3.7)$ \\
$a$ & 0 & parameter in $W_{n p}$ & $a \equiv 2 \pi / n$ \\
$h$ & 0 & warp factor & $(4.6)$ \\
$f$ & -4 & appears in the DBI action & $f=h / T_{3},(5.43)$ \\
$\eta$ & 0 & slow roll parameter & $M_{P l}^{-2} V^{\prime \prime} / V$ \\
$\epsilon$ & 0 & slow roll parameter & $M_{P l}^{-2}\left(V^{\prime} / V\right)^{2}$ \\
\hline
\end{tabular}




\section{Bibliography}

[1] Raffaello Sanzio. Scuola di Atene, (The School of Athens). 15090-1510. Fresco, 500 $770 \mathrm{~cm}$. Vatican City, Apostolic Palace.

[2] J. Polchinski. String theory. Vol. 1: An introduction to the bosonic string. Cambridge, UK: Univ. Pr. (1998) 402 p.

[3] D. Lust and S. Theisen. Lectures on string theory. Springer, Berlin, pages 1-346, 1989.

[4] J. Polchinski. String theory. Vol. 2: Superstring theory and beyond. Cambridge, UK: Univ. Pr. (1998) 531 p.

[5] Michael B. Green, J. H. Schwarz, and Edward Witten. Superstring Theory Vol. 2: Loop Amplitudes, Anomalies and Phenomenology. Cambridge, Uk: Univ. Pr. ( 1987) 596 P. ( Cambridge Monographs On Mathematical Physics).

[6] Michael B. Green, J. H. Schwarz, and Edward Witten. Superstring theory Vol. 1: Introduction. Cambridge, Uk: Univ. Pr. ( 1987) 469 P. ( Cambridge Monographs On Mathematical Physics).

[7] M. Haack. Calabi-Yau fourfold compactifications in string theory. Fortsch. Phys., 50:3-99, 2002.

[8] Steven B. Giddings, Shamit Kachru, and Joseph Polchinski. Hierarchies from fluxes in string compactifications. Phys. Rev., D66:106006, 2002.

[9] T. Kaluza. On the unification problem of physics. HUPD-8401.

[10] O. Klein. Quantum theory and five-dimensional theory of relativity. Z. Phys., 37:895-906, 1926.

[11] P. G. Roll, R. Krotkov, and R. H. Dicke. The Equivalence of inertial and passive gravitational mass. Ann. Phys., 26:442-517, 1964.

[12] T. M. Niebauer, M. P. Mchugh, and J. E. Faller. Galilean Test for the Fifth Force. Phys. Rev. Lett., 59:609-612, 1987.

[13] Steven Weinberg. The quantum theory of fields. Vol. 3: Supersymmetry. Cambridge, UK: Univ. Pr. (2000) 419 p. 
[14] Jonathan Bagger and Julius Wess. Supersymmetry and supergravity. JHU-TIPAC9009.

[15] Sergei Gukov, Cumrun Vafa, and Edward Witten. CFT's from Calabi-Yau fourfolds. Nucl. Phys., B584:69-108, 2000.

[16] Michael R. Douglas and Shamit Kachru. Flux compactification. Rev. Mod. Phys., 79:733-796, 2007.

[17] Mariana Grana. Flux compactifications in string theory: A comprehensive review. Phys. Rept., 423:91-158, 2006.

[18] Lisa Randall and Raman Sundrum. An alternative to compactification. Phys. Rev. Lett., 83:4690-4693, 1999.

[19] Lisa Randall and Raman Sundrum. A large mass hierarchy from a small extra dimension. Phys. Rev. Lett., 83:3370-3373, 1999.

[20] Shamit Kachru, Renata Kallosh, Andrei Linde, and Sandip P. Trivedi. De Sitter vacua in string theory. Phys. Rev., D68:046005, 2003.

[21] Edward Witten. Non-Perturbative Superpotentials In String Theory. Nucl. Phys., B474:343-360, 1996.

[22] K. Choi, A. Falkowski, H. P. Nilles, M. Olechowski, and S. Pokorski. Stability of flux compactifications and the pattern of supersymmetry breaking. JHEP, 11:076, 2004.

[23] Frederik Denef, Michael R. Douglas, Bogdan Florea, Antonella Grassi, and Shamit Kachru. Fixing all moduli in a simple F-theory compactification. Adv. Theor. Math. Phys., 9:861-929, 2005.

[24] Renata Kallosh and Andrei Linde. Landscape, the scale of SUSY breaking, and inflation. JHEP, 12:004, 2004.

[25] Shamit Kachru, John Pearson, and Herman L. Verlinde. Brane/flux annihilation and the string dual of a non- supersymmetric field theory. JHEP, 06:021, 2002.

[26] Raphael Bousso and Joseph Polchinski. Quantization of four-form fluxes and dynamical neutralization of the cosmological constant. JHEP, 06:006, 2000.

[27] Neta A. Bahcall, Jeremiah P. Ostriker, Saul Perlmutter, and Paul J. Steinhardt. The Cosmic Triangle: Revealing the State of the Universe. Science, 284:1481-1488, 1999 .

[28] C. L. Bennett et al. 4-Year COBE DMR Cosmic Microwave Background Observations: Maps and Basic Results. Astrophys. J., 464:L1-L4, 1996. 
[29] D. N. Spergel et al. Wilkinson Microwave Anisotropy Probe (WMAP) three year results: Implications for cosmology. Astrophys. J. Suppl., 170:377, 2007.

[30] E. Komatsu et al. Five-Year Wilkinson Microwave Anisotropy Probe Observations:Cosmological Interpretation. 2008.

[31] Arno A. Penzias and Robert Woodrow Wilson. A Measurement of excess antenna temperature at 4080- Mc/s. Astrophys. J., 142:419-421, 1965. http://nobelprize.org/nobel_prizes/physics/laureates/1978/.

[32] V. Mukhanov. Physical foundations of cosmology. Cambridge, UK: Univ. Pr. (2005) $421 \mathrm{p}$.

[33] A. R. Liddle and D. H. Lyth. Cosmological inflation and large-scale structure. Cambridge, UK: Univ. Pr. (2000) 400 p.

[34] N. Bartolo, E. Komatsu, Sabino Matarrese, and A. Riotto. Non-Gaussianity from inflation: Theory and observations. Phys. Rept., 402:103-266, 2004.

[35] Alan H. Guth. The Inflationary Universe: A Possible Solution to the Horizon and Flatness Problems. Phys. Rev., D23:347-356, 1981.

[36] Alan H. Guth and S. Y. Pi. Fluctuations in the New Inflationary Universe. Phys. Rev. Lett., 49:1110-1113, 1982.

[37] S. W. Hawking. The Development of Irregularities in a Single Bubble Inflationary Universe. Phys. Lett., B115:295, 1982.

[38] Alexei A. Starobinsky. Dynamics of Phase Transition in the New Inflationary Universe Scenario and Generation of Perturbations. Phys. Lett., B117:175-178, 1982.

[39] Andrei D. Linde. A New Inflationary Universe Scenario: A Possible Solution of the Horizon, Flatness, Homogeneity, Isotropy and Primordial Monopole Problems. Phys. Lett., B108:389-393, 1982.

[40] Dalia S. Goldwirth and Tsvi Piran. Initial conditions for inflation. Phys. Rept., 214:223-291, 1992.

[41] Andrei D. Linde. Chaotic Inflation. Phys. Lett., B129:177-181, 1983.

[42] J. H. Taylor and J. M. Weisberg. A new test of general relativity: Gravitational radiation and the binary pulsar PS R 1913+16. Astrophys. J., 253:90-920, 1982.

[43] Joseph H. Taylor and J. M. Weisberg. Further experimental tests of relativistic gravity using the binary pulsar PSR 1913+16. Astrophys. J., 345:434-450, 1989.

[44] S. H. Henry Tye. Brane inflation: String theory viewed from the cosmos. 2006. 
[45] James M. Cline. String cosmology. 2006.

[46] Renata Kallosh. On Inflation in String Theory. Lect. Notes Phys., 738:119-156, 2008.

[47] Andrei Linde. Inflationary Cosmology. Lect. Notes Phys., 738:1-54, 2008.

[48] Liam McAllister and Eva Silverstein. String Cosmology: A Review. Gen. Rel. Grav., 40:565-605, 2008.

[49] G. R. Dvali and S. H. Henry Tye. Brane inflation. Phys. Lett., B450:72-82, 1999.

[50] Mohsen Alishahiha, Eva Silverstein, and David Tong. DBI in the sky. Phys. Rev., D70:123505, 2004.

[51] Eva Silverstein and David Tong. Scalar speed limits and cosmology: Acceleration from D- cceleration. Phys. Rev., D70:103505, 2004.

[52] Jaume Garriga and Viatcheslav F. Mukhanov. Perturbations in k-inflation. Phys. Lett., B458:219-225, 1999.

[53] C. Armendariz-Picon, T. Damour, and Viatcheslav F. Mukhanov. k-inflation. Phys. Lett., B458:209-218, 1999.

[54] Carlos Herdeiro, Shinji Hirano, and Renata Kallosh. String theory and hybrid inflation / acceleration. JHEP, 12:027, 2001.

[55] Keshav Dasgupta, Carlos Herdeiro, Shinji Hirano, and Renata Kallosh. D3/D7 inflationary model and M-theory. Phys. Rev., D65:126002, 2002.

[56] Edmund J. Copeland, Robert C. Myers, and Joseph Polchinski. Cosmic F- and D-strings. JHEP, 06:013, 2004.

[57] Enrico Pajer. Inflation at the Tip. JCAP, 0804:031, 2008.

[58] Joseph P. Conlon and Fernando Quevedo. Kaehler moduli inflation. JHEP, 01:146, 2006.

[59] J. J. Blanco-Pillado et al. Racetrack inflation. JHEP, 11:063, 2004.

[60] J. J. Blanco-Pillado et al. Inflating in a better racetrack. JHEP, 09:002, 2006.

[61] Frederik Denef, Michael R. Douglas, and Bogdan Florea. Building a better racetrack. JHEP, 06:034, 2004.

[62] S. Dimopoulos, S. Kachru, J. McGreevy, and Jay G. Wacker. N-flation. 2005.

[63] Andrew R. Liddle, Anupam Mazumdar, and Franz E. Schunck. Assisted inflation. Phys. Rev., D58:061301, 1998. 
[64] Axel Krause and Enrico Pajer. Chasing Brane Inflation in String-Theory. 2007.

[65] Shamit Kachru et al. Towards inflation in string theory. JCAP, 0310:013, 2003.

[66] Cliff P. Burgess, James M. Cline, Keshav Dasgupta, and Hassan Firouzjahi. Uplifting and inflation with D3 branes. JHEP, 03:027, 2007.

[67] Daniel Baumann, Anatoly Dymarsky, Igor R. Klebanov, Liam McAllister, and Paul J. Steinhardt. A Delicate Universe. Phys. Rev. Lett., 99:141601, 2007.

[68] Daniel Baumann, Anatoly Dymarsky, Igor R. Klebanov, and Liam McAllister. Towards an Explicit Model of D-brane Inflation. JCAP, 0801:024, 2008.

[69] Daniel Baumann et al. On D3-brane potentials in compactifications with fluxes and wrapped D-branes. JHEP, 11:031, 2006.

[70] Marcus Berg, Michael Haack, and Boris Kors. Loop corrections to volume moduli and inflation in string theory. Phys. Rev., D71:026005, 2005.

[71] Daniel Arean, David E. Crooks, and Alfonso V. Ramallo. Supersymmetric probes on the conifold. JHEP, 11:035, 2004.

[72] Peter Ouyang. Holomorphic D7-branes and flavored $\mathrm{N}=1$ gauge theories. Nucl. Phys., B699:207-225, 2004.

[73] S. Kuperstein. Meson spectroscopy from holomorphic probes on the warped deformed conifold. JHEP, 03:014, 2005.

[74] Sergei Gukov. Solitons, superpotentials and calibrations. Nucl. Phys., B574:169$188,2000$.

[75] Oliver DeWolfe, Liam McAllister, Gary Shiu, and Bret Underwood. D3-brane Vacua in Stabilized Compactifications. JHEP, 09:121, 2007.

[76] O. J. Ganor. On zeroes of superpotentials in F-theory. Nucl. Phys. Proc. Suppl., 67:25-29, 1998.

[77] Ori J. Ganor. A note on zeroes of superpotentials in F-theory. Nucl. Phys., B499:5566, 1997.

[78] Paul Koerber and Luca Martucci. From ten to four and back again: how to generalize the geometry. JHEP, 08:059, 2007.

[79] Luca Martucci. D-branes on general N = 1 backgrounds: Superpotentials and Dterms. JHEP, 06:033, 2006.

[80] Oliver DeWolfe and Steven B. Giddings. Scales and hierarchies in warped compactifications and brane worlds. Phys. Rev., D67:066008, 2003. 
[81] C. P. Burgess, R. Kallosh, and F. Quevedo. de Sitter string vacua from supersymmetric D-terms. JHEP, 10:056, 2003.

[82] Philippe Brax, Anne-Christine Davis, Stephen C. Davis, Rachel Jeannerot, and Marieke Postma. Warping and F-term uplifting. JHEP, 09:125, 2007.

[83] Alex Saltman and Eva Silverstein. The scaling of the no-scale potential and de Sitter model building. JHEP, 11:066, 2004.

[84] Rachel Bean, Sarah E. Shandera, S. H. Henry Tye, and Jiajun Xu. Comparing Brane Inflation to WMAP. JCAP, 0705:004, 2007.

[85] Larissa Lorenz. Constraints on brane inflation from WMAP3. 2008.

[86] Larissa Lorenz, Jerome Martin, and Christophe Ringeval. Brane inflation and the WMAP data: a Bayesian analysis. 2007.

[87] Sarah E. Shandera and S. H. Henry Tye. Observing brane inflation. JCAP, 0605:007, 2006.

[88] Liam McAllister. private comunication.

[89] Oliver DeWolfe, Shamit Kachru, and Herman L. Verlinde. The giant inflaton. JHEP, 05:017, 2004.

[90] Gero von Gersdorff and Arthur Hebecker. Kaehler corrections for the volume modulus of flux compactifications. Phys. Lett., B624:270-274, 2005.

[91] Marcus Berg, Michael Haack, and Boris Kors. On volume stabilization by quantum corrections. Phys. Rev. Lett., 96:021601, 2006.

[92] Marcus Berg, Michael Haack, and Enrico Pajer. Jumping Through Loops: On Soft Terms from Large Volume Compactifications. JHEP, 09:031, 2007.

[93] Lev Kofman et al. Beauty is attractive: Moduli trapping at enhanced symmetry points. JHEP, 05:030, 2004.

[94] Liam McAllister and Indrajit Mitra. Relativistic D-brane scattering is extremely inelastic. JHEP, 02:019, 2005.

[95] Damien A. Easson, Ruth Gregory, David F. Mota, Gianmassimo Tasinato, and I. Zavala. Spinflation. JCAP, 0802:010, 2008.

[96] Damien Easson, Ruth Gregory, Gianmassimo Tasinato, and I. Zavala. Cycling in the throat. JHEP, 04:026, 2007.

[97] P. Brax and D. A. Steer. A comment on bouncing and cyclic branes in more than one extra-dimension. 2002. 
[98] Min-xin Huang, Gary Shiu, and Bret Underwood. Multifield DBI Inflation and Non-Gaussianities. Phys. Rev., D77:023511, 2008.

[99] Cristiano Germani, N. E. Grandi, and Alex Kehagias. A stringy alternative to inflation: The cosmological slingshot scenario. 2006.

[100] Cristiano Germani, Nicolas Grandi, and Alex Kehagias. The Cosmological Slingshot Scenario: Myths and Facts. 2007.

[101] Cristiano Germani and Michele Liguori. Matching WMAP 3-yrs results with the Cosmological Slingshot Primordial Spectrum. 2007.

[102] Cristiano Germani, Nicolas Grandi, and Alex Kehagias. The Cosmological Slingshot Scenario: a Stringy Proposal for the Early Time Cosmology. 2008.

[103] Christopher Savage, Katherine Freese, and William H. Kinney. Natural inflation: Status after WMAP 3-year data. Phys. Rev., D74:123511, 2006.

[104] Katherine Freese, Joshua A. Frieman, and Angela V. Olinto. Natural inflation with pseudo - Nambu-Goldstone bosons. Phys. Rev. Lett., 65:3233-3236, 1990.

[105] Kazunori Kohri, Chia-Min Lin, and David H. Lyth. More hilltop inflation models. JCAP, 0712:004, 2007.

[106] Lotfi Boubekeur and David. H. Lyth. Hilltop inflation. JCAP, 0507:010, 2005.

[107] Igor R. Klebanov and Matthew J. Strassler. Supergravity and a confining gauge theory: Duality cascades and chiSB-resolution of naked singularities. JHEP, 08:052, 2000 .

[108] Philip Candelas and Xenia C. de la Ossa. Comments on Conifolds. Nucl. Phys., B342:246-268, 1990.

[109] A. Achucarro, B. de Carlos, J. A. Casas, and L. Doplicher. de Sitter vacua from uplifting D-terms in effective supergravities from realistic strings. JHEP, 06:014, 2006.

[110] D. Cremades, M. P. Garcia del Moral, F. Quevedo, and K. Suruliz. Moduli stabilisation and de Sitter string vacua from magnetised D7 branes. JHEP, 05:100, 2007.

[111] Hans Jockers and Jan Louis. D-terms and F-terms from D7-brane fluxes. Nucl. Phys., B718:203-246, 2005.

[112] Kiwoon Choi, Adam Falkowski, Hans Peter Nilles, and Marek Olechowski. Soft supersymmetry breaking in KKLT flux compactification. Nucl. Phys., B718:113$133,2005$. 
[113] P. G. Camara, L. E. Ibanez, and A. M. Uranga. Flux-induced SUSY-breaking soft terms on D7-D3 brane systems. Nucl. Phys., B708:268-316, 2005.

[114] Michael Haack, Daniel Krefl, Dieter Lust, Antoine Van Proeyen, and Marco Zagermann. Gaugino condensates and D-terms from D7-branes. JHEP, 01:078, 2007.

[115] D. Lust, P. Mayr, S. Reffert, and S. Stieberger. F-theory flux, destabilization of orientifolds and soft terms on D7-branes. Nucl. Phys., B732:243-290, 2006.

[116] Keshav Dasgupta, Paul Franche, Anke Knauf, and James Sully. D-terms on the resolved conifold. 2008.

[117] Hiroyuki Abe, Tetsutaro Higaki, and Tatsuo Kobayashi. More about F-term uplifting. Phys. Rev., D76:105003, 2007.

[118] Z. Lalak, O. J. Eyton-Williams, and R. Matyszkiewicz. F-term uplifting via consistent D-terms. JHEP, 05:085, 2007.

[119] Susha L. Parameswaran and Alexander Westphal. Consistent de Sitter string vacua from Kaehler stabilization and D-term uplifting. Fortsch. Phys., 55:804-810, 2007.

[120] Hiroyuki Abe, Tetsutaro Higaki, Tatsuo Kobayashi, and Yuji Omura. Moduli stabilization, F-term uplifting and soft supersymmetry breaking terms. Phys. Rev., D75:025019, 2007.

[121] Emilian Dudas, Chloe Papineau, and Stefan Pokorski. Moduli stabilization and uplifting with dynamically generated F-terms. JHEP, 02:028, 2007.

[122] Aalok Misra and Pramod Shukla. Large Volume Axionic Swiss-Cheese Inflation. 2007.

[123] Aalok Misra and Pramod Shukla. Area Codes, Large Volume (Non-)Perturbative alpha'- and Instanton - Corrected Non-supersymmetric (A)dS minimum, the Inverse Problem and Fake Superpotentials for Multiple- Singular-Loci-Two-Parameter Calabi-Yau's. 2007.

[124] Oleg Lebedev, Hans Peter Nilles, and Michael Ratz. de Sitter vacua from matter superpotentials. Phys. Lett., B636:126-131, 2006.

[125] Marta Gomez-Reino and Claudio A. Scrucca. Locally stable non-supersymmetric Minkowski vacua in supergravity. JHEP, 05:015, 2006.

[126] Oleg Lebedev, Valeri Lowen, Yann Mambrini, Hans Peter Nilles, and Michael Ratz. Metastable vacua in flux compactifications and their phenomenology. JHEP, 02:063, 2007.

[127] Herman L. Verlinde. Holography and compactification. Nucl. Phys., B580:264-274, 2000 . 
[128] Thomas W. Grimm. Axion Inflation in Type II String Theory. 2007.

[129] Renata Kallosh, Navin Sivanandam, and Masoud Soroush. Axion Inflation and Gravity Waves in String Theory. Phys. Rev., D77:043501, 2008.

[130] Tom Banks, Michael Dine, Patrick J. Fox, and Elie Gorbatov. On the possibility of large axion decay constants. JCAP, 0306:001, 2003.

[131] Martin E. Olsson. Inflation Assisted by Heterotic Axions. JCAP, 0704:019, 2007.

[132] Katrin Becker, Melanie Becker, and Axel Krause. M-theory inflation from multi M5-brane dynamics. Nucl. Phys., B715:349-371, 2005.

[133] Michael Dine and Antonio Riotto. An inflaton candidate in gauge mediated supersymmetry breaking. Phys. Rev. Lett., 79:2632-2635, 1997.

[134] Steven Kecskemeti, John Maiden, Gary Shiu, and Bret Underwood. DBI inflation in the tip region of a warped throat. JHEP, 09:076, 2006.

[135] Bret Underwood. Brane Inflation is Attractive. 2008.

[136] Xingang Chen. Inflation from warped space. JHEP, 08:045, 2005.

[137] Daniel Baumann and Liam McAllister. A microscopic limit on gravitational waves from D-brane inflation. Phys. Rev., D75:123508, 2007.

[138] David H. Lyth. What would we learn by detecting a gravitational wave signal in the cosmic microwave background anisotropy? Phys. Rev. Lett., 78:1861-1863, 1997.

[139] Rouzbeh Allahverdi, Kari Enqvist, Juan Garcia-Bellido, Asko Jokinen, and Anupam Mazumdar. MSSM flat direction inflation: slow roll, stability, fine tunning and reheating. JCAP, 0706:019, 2007.

[140] Sudhakar Panda, M. Sami, and Shinji Tsujikawa. Prospects of inflation in delicate D-brane cosmology. Phys. Rev., D76:103512, 2007.

[141] Anatoly Dymarsky, Igor R. Klebanov, and Nathan Seiberg. On the moduli space of the cascading $\mathrm{SU}(\mathrm{M}+\mathrm{p}) \times \mathrm{SU}(\mathrm{p})$ gauge theory. JHEP, 01:155, 2006.

[142] Alex Buchel. Inflation on the resolved warped deformed conifold. Phys. Rev., D74:046009, 2006.

[143] Joseph P. Conlon, Fernando Quevedo, and Kerim Suruliz. Large-volume flux compactifications: Moduli spectrum and D3/D7 soft supersymmetry breaking. JHEP, 08:007, 2005.

[144] Joseph P. Conlon and Fernando Quevedo. Gaugino and scalar masses in the landscape. JHEP, 06:029, 2006. 
[145] Joseph P. Conlon, Shehu S. Abdussalam, Fernando Quevedo, and Kerim Suruliz. Soft SUSY breaking terms for chiral matter in IIB string compactifications. JHEP, 01:032, 2007.

[146] Joseph P. Conlon. The QCD axion and moduli stabilisation. JHEP, 05:078, 2006.

[147] Joseph P. Conlon. Seeing the invisible axion in the sparticle spectrum. Phys. Rev. Lett., 97:261802, 2006.

[148] Joseph P. Conlon and Daniel Cremades. The neutrino suppression scale from large volumes. Phys. Rev. Lett., 99:041803, 2007.

[149] Gordon L. Kane, Piyush Kumar, and Jing Shao. LHC String Phenomenology. J. Phys., G34:1993-2036, 2007.

[150] R. Holman and Jimmy A. Hutasoit. Axionic inflation from large volume flux compactifications. 2006.

[151] Joan Simon, Raul Jimenez, Licia Verde, Per Berglund, and Vijay Balasubramanian. Using cosmology to constrain the topology of hidden dimensions. 2006.

[152] J. Richard Bond, Lev Kofman, Sergey Prokushkin, and Pascal M. Vaudrevange. Roulette inflation with Kaehler moduli and their axions. Phys. Rev., D75:123511, 2007.

[153] Lars Gorlich, Shamit Kachru, Prasanta K. Tripathy, and Sandip P. Trivedi. Gaugino condensation and nonperturbative superpotentials in flux compactifications. JHEP, 12:074, 2004.

[154] Prasanta K. Tripathy and Sandip P. Trivedi. D3 brane action and fermion zero modes in presence of background flux. JHEP, 06:066, 2005.

[155] Natalia Saulina. Topological constraints on stabilized flux vacua. Nucl. Phys., B720:203-210, 2005.

[156] Renata Kallosh, Amir-Kian Kashani-Poor, and Alessandro Tomasiello. Counting fermionic zero modes on M5 with fluxes. JHEP, 06:069, 2005.

[157] Luca Martucci, Jan Rosseel, Dieter Van den Bleeken, and Antoine Van Proeyen. Dirac actions for D-branes on backgrounds with fluxes. Class. Quant. Grav., 22:2745-2764, 2005.

[158] P. Berglund and P. Mayr. Non-perturbative superpotentials in F-theory and string duality. 2005.

[159] Eric Bergshoeff, Renata Kallosh, Amir-Kian Kashani-Poor, Dmitri Sorokin, and Alessandro Tomasiello. An index for the Dirac operator on D3 branes with background fluxes. JHEP, 10:102, 2005. 
[160] Dieter Lust, Susanne Reffert, Waldemar Schulgin, and Prasanta K. Tripathy. Fermion zero modes in the presence of fluxes and a non- perturbative superpotential. JHEP, 08:071, 2006.

[161] D. Lust, S. Reffert, E. Scheidegger, W. Schulgin, and S. Stieberger. Moduli stabilization in type IIB orientifolds. II. Nucl. Phys., B766:178-231, 2007.

[162] Ralph Blumenhagen, Mirjam Cvetic, and Timo Weigand. Spacetime instanton corrections in $4 \mathrm{D}$ string vacua - the seesaw mechanism for D-brane models. Nucl. Phys., B771:113-142, 2007.

[163] Nikolas Akerblom, Ralph Blumenhagen, Dieter Lust, Erik Plauschinn, and Maximilian Schmidt-Sommerfeld. Non-perturbative SQCD Superpotentials from String Instantons. JHEP, 04:076, 2007.

[164] Dimitrios Tsimpis. Fivebrane instantons and Calabi-Yau fourfolds with flux. JHEP, 03:099, 2007.

[165] Massimo Bianchi and Elias Kiritsis. Non-perturbative and Flux superpotentials for Type I strings on the Z3 orbifold. Nucl. Phys., B782:26-50, 2007.

[166] Riccardo Argurio, Matteo Bertolini, Gabriele Ferretti, Alberto Lerda, and Christoffer Petersson. Stringy Instantons at Orbifold Singularities. JHEP, 06:067, 2007.

[167] D. Lust, S. Reffert, W. Schulgin, and S. Stieberger. Moduli stabilization in type IIB orientifolds. I: Orbifold limits. Nucl. Phys., B766:68-149, 2007.

[168] Daniel Krefl and Dieter Lust. On supersymmetric Minkowski vacua in IIB orientifolds. JHEP, 06:023, 2006.

[169] Vijay Balasubramanian, Per Berglund, Joseph P. Conlon, and Fernando Quevedo. Systematics of moduli stabilisation in Calabi-Yau flux compactifications. JHEP, 03:007, 2005.

[170] Katrin Becker, Melanie Becker, Michael Haack, and Jan Louis. Supersymmetry breaking and alpha'-corrections to flux induced potentials. JHEP, 06:060, 2002.

[171] Marcus Berg, Michael Haack, and Boris Kors. String loop corrections to Kaehler potentials in orientifolds. JHEP, 11:030, 2005.

[172] Ignatios Antoniadis, C. Bachas, C. Fabre, H. Partouche, and T. R. Taylor. Aspects of type I - type II - heterotic triality in four dimensions. Nucl. Phys., B489:160-178, 1997.

[173] Carlo Angelantonj and Augusto Sagnotti. Open strings. Phys. Rept., 371:1-150, 2002.

[174] C. P. Burgess et al. Warped supersymmetry breaking. 2006. 
[175] Steven B. Giddings and Anshuman Maharana. Dynamics of warped compactifications and the shape of the warped landscape. Phys. Rev., D73:126003, 2006.

[176] E. Cremmer, S. Ferrara, C. Kounnas, and Dimitri V. Nanopoulos. Naturally Vanishing Cosmological Constant in N=1 Supergravity. Phys. Lett., B133:61, 1983.

[177] Philip Candelas, Anamaria Font, Sheldon H. Katz, and David R. Morrison. Mirror symmetry for two parameter models. 2. Nucl. Phys., B429:626-674, 1994.

[178] Gottfried Curio and Vera Spillner. On the modified KKLT procedure: A case study for the $\mathrm{P}(11169)(18)$ model. Int. J. Mod. Phys., A22:3463-3492, 2007.

[179] Michael Dine and Nathan Seiberg. Is the Superstring Weakly Coupled? Phys. Lett., B162:299, 1985.

[180] Vijay Balasubramanian and Per Berglund. Stringy corrections to Kaehler potentials, SUSY breaking, and the cosmological constant problem. JHEP, 11:085, 2004.

[181] Ralph Blumenhagen, Boris Kors, Dieter Lust, and Stephan Stieberger. Fourdimensional String Compactifications with D-Branes, Orientifolds and Fluxes. Phys. Rept., 445:1-193, 2007.

[182] Michael B. Green. Interconnections between type II superstrings, M theory and N $=4$ Yang-Mills. 1999 .

[183] Kiwoon Choi and Hans Peter Nilles. The gaugino code. JHEP, 04:006, 2007.

[184] Hans Peter Nilles. Supersymmetry, Supergravity and Particle Physics. Phys. Rept., 110:1, 1984.

[185] Stephen P. Martin. A supersymmetry primer. 1997.

[186] Vadim S. Kaplunovsky. One Loop Threshold Effects in String Unification. Nucl. Phys., B307:145, 1988.

[187] Joseph P. Conlon, Daniel Cremades, and Fernando Quevedo. Kaehler potentials of chiral matter fields for Calabi-Yau string compactifications. JHEP, 01:022, 2007.

[188] David Berenstein. Branes vs. GUTS: Challenges for string inspired phenomenology. 2006.

[189] Luis E. Ibanez, F. Marchesano, and R. Rabadan. Getting just the standard model at intersecting branes. JHEP, 11:002, 2001.

[190] Philip Candelas, Xenia De La Ossa, Anamaria Font, Sheldon H. Katz, and David R. Morrison. Mirror symmetry for two parameter models. I. Nucl. Phys., B416:481538, 1994. 
[191] Michael B. Green, Jeffrey A. Harvey, and Gregory W. Moore. I-brane inflow and anomalous couplings on D-branes. Class. Quant. Grav., 14:47-52, 1997.

[192] Keshav Dasgupta, Dileep P. Jatkar, and Sunil Mukhi. Gravitational couplings and Z(2) orientifolds. Nucl. Phys., B523:465-484, 1998.

[193] Yeuk-Kwan E. Cheung and Zheng Yin. Anomalies, branes, and currents. Nucl. Phys., B517:69-91, 1998.

[194] Ruben Minasian and Gregory W. Moore. K-theory and Ramond-Ramond charge. JHEP, 11:002, 1997.

[195] Jose F. Morales, Claudio A. Scrucca, and Marco Serone. Anomalous couplings for D-branes and O-planes. Nucl. Phys., B552:291-315, 1999.

[196] Jr. Stefanski, Bogdan. Gravitational couplings of D-branes and O-planes. Nucl. Phys., B548:275-290, 1999.

[197] Constantin P. Bachas, Pascal Bain, and Michael B. Green. Curvature terms in D-brane actions and their M-theory origin. JHEP, 05:011, 1999.

[198] A. Fotopoulos. On (alpha')**2 corrections to the D-brane action for non- geodesic world-volume embeddings. JHEP, 09:005, 2001.

[199] E. Dudas, G. Pradisi, M. Nicolosi, and A. Sagnotti. On tadpoles and vacuum redefinitions in string theory. Nucl. Phys., B708:3-44, 2005.

[200] Ruben Minasian and Dimitrios Tsimpis. On the geometry of non-trivially embedded branes. Nucl. Phys., B572:499-513, 2000.

[201] Ofer Aharony, Yaron E. Antebi, and Micha Berkooz. Open string moduli in KKLT compactifications. Phys. Rev., D72:106009, 2005.

[202] Nemanja Kaloper and Robert C. Myers. The O(dd) story of massive supergravity. JHEP, 05:010, 1999.

[203] Shamit Kachru, Michael B. Schulz, and Sandip Trivedi. Moduli stabilization from fluxes in a simple IIB orientifold. JHEP, 10:007, 2003.

[204] Marcus Berg, Michael Haack, and Boris Kors. On the moduli dependence of nonperturbative superpotentials in brane inflation. 2004.

[205] D. Lust, P. Mayr, R. Richter, and S. Stieberger. Scattering of gauge, matter, and moduli fields from intersecting branes. Nucl. Phys., B696:205-250, 2004.

[206] C. P. Burgess and T. R. Morris. Open and unoriented strings a la Polyakov. Nucl. Phys., B291:256, 1987.

[207] C. P. Burgess and T. R. Morris. Open superstrings a la Polyakov. Nucl. Phys., B291:285, 1987. 